\title{
Quantum 4d Yang-Mills theory and time-reversal symmetric 5d higher-gauge topological field theory
}

\author{
Zheyan Wan, ${ }^{1,2, \dagger}$ Juven Wang $\oplus^{3,4, *}$ and Yunqin Zheng ${ }^{5, *}$ \\ ${ }^{1}$ Yau Mathematical Sciences Center, Tsinghua University, Beijing 100084, China \\ ${ }^{2}$ School of Mathematical Sciences, USTC, Hefei 230026, China \\ ${ }^{3}$ School of Natural Sciences, Institute for Advanced Study, Einstein Drive, Princeton, \\ New Jersey 08540, USA \\ ${ }^{4}$ Center of Mathematical Sciences and Applications, Harvard University, Cambridge, \\ Massachusetts 02138, USA \\ ${ }^{5}$ Physics Department, Princeton University, Princeton, New Jersey 08544, USA
}

(Received 14 May 2019; revised manuscript received 24 August 2019; published 21 October 2019; corrected 31 October 2019)

\begin{abstract}
We explore various 4d Yang-Mills gauge theories (YM) living as boundary conditions of 5d gapped short-/long-range entangled (SRE/LRE) topological states. Specifically, we explore 4d time-reversal symmetric pure $\mathrm{YM}$ of an $\mathrm{SU}(2)$ gauge group with a second-Chern-class topological term at $\theta=\pi$ $\left[\mathrm{SU}(2)_{\theta=\pi} \mathrm{YM}\right]$, by turning on the background fields for both the time reversal (i.e., on unorientable manifolds) and the one-form center global symmetry. We find four siblings of $\mathrm{SU}(2)_{\theta=\pi} \mathrm{YM}$ with distinct couplings to background fields, labeled by $\left(K_{1}, K_{2}\right): K_{1}=0,1$ specifies Kramers singlet/doublet Wilson line and new mixed higher 't Hooft anomalies; $K_{2}=0,1$ specifies the boson/fermionic Wilson line and a new Wess-Zumino-Witten-like counterterm. Higher anomalies indicate that to realize all higher $n$-global symmetries locally on $n$ simplices, the $4 \mathrm{~d}$ theory becomes a boundary of a $5 \mathrm{~d}$ higher-symmetry-protected topological state (SPTs, as an invertible topological quantum field theory (iTQFT) or a cobordism invariant in math, or as a $5 \mathrm{~d}$ higher-symmetric interacting topological superconductor in condensed matter). Via Weyl's gauge principle, by dynamically gauging the one-form symmetry, we transform 5d bulk SRE SPTs into an LRE symmetry-enriched topologically ordered state (SETs); thus we obtain the $4 \mathrm{~d} \mathrm{SO}(3)_{\theta=\pi}$ YM5d LRE-higher-SETs coupled system with dynamical higher-form gauge fields. We further derive new exotic anyonic statistics of extended objects such as two world sheets of strings and three world volumes of branes, physically characterizing the 5d SETs. We discover triple and quadruple link invariants potentially associated with the underlying $5 \mathrm{~d}$ higher-gauge topological quantum field theories, hinting at a new intrinsic relation between nonsupersymmetric 4d pure YM and topological links in 5d. We provide 4d-5d lattice simplicial complex regularizations and bridge to 4d SU(2)- and $\mathrm{SO}(3)$-gauged quantum spin liquids as $(3+1)$-dimensional realizations.
\end{abstract}

DOI: 10.1103/PhysRevD.100.085012

\section{INTRODUCTION AND SUMMARY}

The world where we reside, to our best present understanding, can be described by quantum theory and the underlying long-range entanglement. Quantum field theory (QFT) and in particular quantum gauge field theory, under the spell of the gauge principle following the insights since

\footnotetext{
${ }^{*}$ Corresponding author. juven@ias.edu

†wanzy@mail.ustc.edu.cn

‡yunqinz@princeton.edu
}

Published by the American Physical Society under the terms of the Creative Commons Attribution 4.0 International license. Further distribution of this work must maintain attribution to the author(s) and the published article's title, journal citation, and DOI. Funded by SCOAP ${ }^{3}$.
Maxwell, Hilbert, Weyl, Pauli, and others (see a historical review [1]), embodies the quantum, special relativity, and long-range entanglement into a systematic framework. Yang-Mills (YM) gauge theory [2], generalizing the $\mathrm{U}(1)$ gauge group of quantum electrodynamics to a nonAbelian Lie group, has been proven to be powerful to describe the Standard Model physics.

The Euclidean partition function of a pure YM theory with an SU(N) gauge group on a four-dimensional (i.e., $4 d)^{1}$ spacetime $M^{4}$ and a second-Chern-class topological term $S_{\theta}$ labeled by $\theta$, i.e., $\mathrm{SU}(\mathrm{N})_{\theta}$-YM, is

\footnotetext{
${ }^{1}$ We denote $n$ d for an $n$-dimensional spacetime. We denote $m+1 \mathrm{D}$ for an $m$-dimensional space and one-dimensional time. We denote $m \mathrm{D}$ for an $m$-dimensional spatial object.
} 


$$
\begin{aligned}
& \mathbf{Z}_{\mathrm{YM}}^{4 \mathrm{~d}} \equiv \int[\mathcal{D} a] \exp \left(-S_{\mathrm{SU}(\mathrm{N})_{\theta}}[a]\right) \\
& \equiv \int[\mathcal{D} a] \exp \left(-S_{\mathrm{SU}(\mathrm{N})_{\theta=0}}[a]\right) \exp \left(-S_{\theta}[a]\right) \\
& \equiv \int[\mathcal{D} a] \exp \left(-\int_{M^{4}} \frac{1}{g^{2}} \operatorname{Tr} F_{a} \wedge \star F_{a}\right. \\
&\left.+\int_{M^{4}} \frac{\mathrm{i} \theta}{8 \pi^{2}} \operatorname{Tr} F_{a} \wedge F_{a}\right)
\end{aligned}
$$

where $a$ is the $\mathrm{SU}(\mathrm{N})$ gauge field and $F_{a}=\mathrm{d} a-\mathrm{i} a \wedge a$ is the SU(N) field strength. $g$ is the gauge coupling constant. See the footnote ${ }^{2}$ for further explanations of the notations. When $\theta=0$, the $\mathrm{SU}(\mathrm{N})_{\theta=0} \mathrm{YM}$ theory is believed to be in the confined phase [3], to have an energy gap, and to have a single ground state on any manifold. In particular, there is no 't Hooft anomaly [4]. Recently, the authors of Ref. [5] discovered that for $\mathrm{SU}(\mathrm{N})_{\theta=\pi}-\mathrm{YM}$ with even $\mathrm{N}$, there is a subtle 't Hooft anomaly [4] involving the time-reversal symmetry $\mathbb{Z}_{2}^{T}$ and one-form center global symmetry [6]

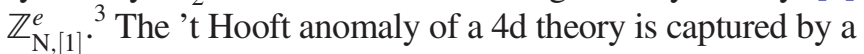
$5 \mathrm{~d}$ topological term through the anomaly inflow [7] mechanism. Schematically, Ref. [5] suggested a 5d topological term linear in the time-reversal background field $\mathcal{T}$ and quadratic in the $\mathbb{Z}_{\mathrm{N},[1]}^{e}$ background field $B$,

$$
\sim \mathcal{T} B B
$$

The $5 \mathrm{~d}$ topological term characterizes the $5 \mathrm{~d}$ short-rangeentangled (SRE) phase. See Sec. II for more rigorous definitions of the background fields and the $5 \mathrm{~d}$ topological term.

\footnotetext{
${ }^{2} a$ is locally a one-form SU(N) connection obtained from parallel transporting the principal-SU(N) bundle over the spacetime manifold $M^{4}$. Locally $a=a_{\mu} \mathrm{d} x^{\mu}=a_{\mu}^{\alpha} T^{\alpha} \mathrm{d} x^{\mu}$ with $T^{\alpha}$ is the generator of $\mathrm{su}(\mathrm{N})$ Lie algebra, satisfying the commutator $\left[T^{\alpha}, T^{\beta}\right]=\mathrm{i} f^{\alpha \beta \gamma} T^{\gamma}$ where $f^{\alpha \beta \gamma}$ is a fully antisymmetric structure constant. Locally $\mathrm{d} x^{\mu}$ is a differential one-form. $a_{\mu}=a_{\mu}^{\alpha} T^{\alpha}$ is the Lie algebra valued gauge field. The path integral $\int[\mathcal{D} a]$ is meant to integrate over all the configurations of $a(t, x)$ modding out the $\mathrm{SU}(\mathrm{N})$ gauge transformation $a \rightarrow g^{-1} a g-\mathrm{i} g^{-1} \mathrm{~d} g$. The $\frac{1}{g^{2}} \operatorname{Tr}\left(F_{a} \wedge\right.$ $\left.\star F_{a}\right)$ is the Yang-Mills Lagrangian [2] [a non-Abelian generalization of Maxwell U(1) gauge theory] where $\star F_{a}$ is the Hodge dual of $F_{a}$. Tr stands for the trace as an invariant quadratic form of the Lie algebra su(N). The term $S_{\theta}[a]=\frac{-\mathrm{i} \theta}{8 \pi^{2}} \operatorname{Tr} F_{a} \wedge F_{a}$ is related to the second-Chern-class $c_{2}\left(V_{\mathrm{SU}(\mathrm{N})}\right)$ of the $\mathrm{SU}(\mathrm{N})$ gauge bundle via $S_{\theta}[a]=-\mathrm{i} \theta c_{2}\left(V_{\mathrm{SU}(\mathrm{N})}\right)$. This path integral is sensible for physicists, but may not be mathematically well defined. We will also point out how to grasp the meaning of the YM path integral on unorientable manifolds in Sec. II.

${ }^{3} \mathrm{We}$ use the subscript [1] to indicate that the symmetry is one-form, and the superscript $e$ to indicate the symmetry is electric as opposed to magnetic (i.e., the charged line operators are the Wilson lines rather than the 't Hooft lines). When we say symmetry in this article, we always mean global symmetry unless we state otherwise.
}

Further recently, Ref. [8] suggested that there are additional new higher 't Hooft anomalies for some $4 \mathrm{~d}$ $\mathrm{SU}(\mathrm{N})_{\theta=\pi}$ theories at even $\mathrm{N}$ : From one perspective, Ref. [8] suggested that when $\mathrm{N}=2$, there is a mixed anomaly captured by a $5 \mathrm{~d}$ topological term which is cubic in $\mathcal{T}$ and linear in $B$, which is schematically denoted as

$$
\sim \mathcal{T} \mathcal{T} \mathcal{T} B
$$

From another perspective, Ref. [8] suggested that the $\mathrm{SU}(\mathrm{N})_{\theta=\pi} \mathrm{YM}$ at an even integer $\mathrm{N} \geq 4$ contains new mixed anomalies involving $\mathbb{Z}_{2}^{T}, \mathbb{Z}_{\mathrm{N},[1]}^{e}$, and a zero-form charge conjugation (i.e., a $\mathbb{Z}_{2}$ outer-automorphism) symmetry. For example, at $N=4$, another anomaly can be captured by the $5 \mathrm{~d}$ topological terms schematically as

$$
\sim A_{C} \mathcal{T} \mathcal{T} B
$$

The detailed derivation will be left for the future work [9]. ${ }^{4}$ Here $A_{C}$ is a one-form background field for the zero-form $\mathbb{Z}_{2}^{C}$ charge conjugation symmetry. In the following, we will make the above schematic $5 \mathrm{~d}$ topological terms Eqs. (1.2), (1.3), and (1.4) mathematically precise, following the setup in Refs. [8,10].

The above $5 \mathrm{~d}$ topological terms can be regarded as semiclassical partition functions (definable on closed five manifolds with appropriate structures) whose functional values depend on the couplings to global symmetrybackground probe fields. These $5 \mathrm{~d}$ topological terms are also known as invertible topological quantum field theories (iTQFTs) in the literature. ${ }^{5}$ In the present work, we will further dynamically gauge the one-form symmetry $\mathbb{Z}_{\mathrm{N},[1]}^{e}$ associated with the coupled systems of $4 \mathrm{~d} Y M$ and $5 \mathrm{~d}$ topological terms above. After gauging, the 5d SRE topological terms become $5 \mathrm{~d}$ long-range entangled (LRE) topological quantum field theories (TQFT). We further apply the methods developed in Refs. [11-13] to analytically compute the physical observables of the

\footnotetext{
${ }^{4}$ Note that so far for $N=4$, we only find a new $4 \mathrm{~d}$ anomaly expressible by a term

$$
A_{C} w_{1}(T M)^{2} B \sim A_{C} \mathcal{T} \mathcal{T} B
$$

Although there is still a possibility that another $4 \mathrm{~d}$ anomaly may exist

$$
A_{C}^{2} \beta_{(2,4)} B \sim A_{C} A_{C} \mathcal{T} B
$$

More precisely, these two $4 \mathrm{~d}$ anomalies are captured by $5 \mathrm{~d}$ invertible topological quantum field theories (iTQFTs) $A_{C} w_{1}(T M)^{2} B$ and $A_{C}^{2} \beta_{(2,4)} B$ on a $5 d$ closed manifold.

${ }^{5}$ By iTQFT, physically it means that the absolute value of partition function $|\mathbf{Z}|=1$ on any closed manifold. Thus this $\mathbf{Z}$ can only be a complex phase $\mathbf{Z}=\mathrm{e}^{\mathrm{i} \theta}$, which can thus be inverted and canceled by $\mathrm{e}^{-\mathrm{i} \theta}$ as another iTQFT.
} 
higher-gauge 5d TQFTs. The physical observables of $5 \mathrm{~d}$ TQFTs include, for example, (i) the partition functions $\mathbf{Z}\left[M^{5}\right]$ on closed manifolds $M^{5}$, and (ii) braiding statistics of anyonic strings and anyonic branes whose spacetime trajectories form two world sheets and three world volumes, respectively, and link invariants of these two world sheets and three world volumes in $M^{5}$. We uncover new spacetime braiding processes and link invariants, including triple and quadruple linkings analogous to previous works $[11,12,14-16] .^{6}$

Now let us take a step back to digest the physical meanings of these $5 \mathrm{~d}$ topological terms Eq. (1.2)-Eq. (1.4). The $d$-dimensional 't Hooft anomaly of ordinary zeroform global symmetries is known to be captured by a $(d+1)$-dimensional iTQFT. In the condensed matter literatures, these $(d+1) \mathrm{d}$ iTQFTs describe symmetryprotected topological states (SPTs) ${ }^{7}$ [29-32]. The relations between the SPTs and the response probe field-theoretic partition functions have been systematically studied, selectively, in [21,33-38] (and references therein), and climaxed to the hint of cobordism classification of SPTs $[39,40]$. Recently the iTQFTs and SPTs have been found to be systematically classified by a powerful cobordism theory framework of Freed-Hopkins [41], following the earlier work of Thom-Madsen-Tillmann spectra [42,43].

Further recently, Ref. [10] generalized the Thom-MadsenTillmann-Freed-Hopkins cobordism theory [41-43] to the cases with generalized higher global symmetries [6]. The generalized cobordism group computation [10], which involves the bordism group of higher classifying spaces and their fibrations, e.g., $\mathrm{B} \mathbb{G}$, can capture the $d$-dimensional higher 't Hooft anomaly of generalized global symmetries $\mathbb{G}$ by $(d+1)$-dimensional bordism invariants (i.e., generalized symmetric or higher symmetric iTQFTs). In the following, ${ }^{6}$ Here we comment on the physical and mathematical meanings of fractional statistics and non-Abelian statistics associated with the
spacetime braiding processes involving 0D anyonic particles, 1D anyonic strings, 2D anyonic branes, and other extended objects. In the
discussions below, we take a generalized definition of anyonic:

(i) In a more restricted definition, anyonic means the self-exchange statistics can go beyond bosons or fermions [17].

(ii) In our generalized definition, anyonic means that either self-exchange statistics (of identical objects) or the mutual statistics (of multiple distinguishable objects, may involving more than two objects) can go beyond bosonic or fermionic statistics.--In 3d $(2+1 D)$ spacetime $M^{3}$, braiding statistics of particles can be fractional (such as the exchange statistics of two identical particles, or mutual statistics of two different particles) which are called anyonic particles (see an excellent historical overview [17]). As an example, this can be understood from a $3 \mathrm{~d}$ Chern-Simons action with one-form gauge field $a$ integrated over $M^{3}$ $\sim \int_{M^{3}} a_{I} \mathrm{~d} a_{J}$, which modifies the quantum statistics of particle world line whose open ends host the anyonic particles.-In $4 \mathrm{~d}(3+1 \mathrm{D})$ spacetime $M^{4}$, braiding statistics of particles cannot be fractional as the two 1-world lines cannot be linked in 4d. Thus there is no anyonic particle and no fractional particle statistics beyond bosons or fermions in 4d. However, the braiding statistics of strings can be fractional, which we dub anyonic strings. As an example, the fractional statistics of strings can be understood from a $4 \mathrm{~d}$ TQFT with a one-form gauge field $a$ and a two-form gauge field $b$, as $\sim \int_{M^{4}} b \mathrm{~d} a$, which modifies the mutual quantum statistics of a OD particle from one world line $W_{1}=e^{i \int_{\gamma} a}$ linked with a $1 \mathrm{D}$ string from two world sheet $U_{2}=e^{i \int_{S^{2}} b}$ in $M^{4}$. Since a particle cannot carry a fractional charge in $4 \mathrm{~d}$, we can interpret the above theory as the anyonic string carrying a fractional flux in $4 \mathrm{~d}$. Another way to interpret the fractional statistics of anyonic strings is through the dimensional reduction from $4 \mathrm{~d}$ to $3 \mathrm{~d}$. Let $M^{4}=S^{1} \times M^{3}$ with the size of $S^{1}$ much smaller than the size of $M^{3}$ and let the closed anyonic strings wrap around $S^{1}$ [18-20]; then the anyonic strings in $M^{4}$ reduce to anyonic particles in $M^{3}$. From the field theory side, the $4 \mathrm{~d}$ TQFTs $\sim \int_{M^{4}} a_{I} a_{J} \mathrm{~d} a_{K}, \sim \int_{M^{4}} a_{I} a_{J} a_{K} a_{L}$ can modify the braiding statistics of strings [11,12,21-26]. See the relations between Dijkgraaf-Witten's group cohomology theory [27] and these TQFTs discussed in [11,12,21]. Furthermore, there are 4d gauge invariant topological terms with two-form gauge field $b[6,12,28] \sim \int_{M^{4}} b_{I} b_{J}$. - In $5 \mathrm{~d}(4+1 \mathrm{D})$ spacetime $M^{5}$, for example, there exist self- and mutual coupling types of $5 \mathrm{~d}$ topological terms with two-form gauge fields $b, b_{I}, b_{J}$, etc., $\sim \int_{M^{5}} b \mathrm{~d} b, \sim \int_{M^{5}} b_{I} \mathrm{~d} b_{J}$. The self-coupling term $\int_{M^{5}} b \mathrm{~d} b$ leads to anyonic strings within the restricted definition, where the self-exchange statistics goes beyond bosonic and fermionic [17]. The mutual coupling term $\int_{M^{5}} b_{I} \mathrm{~d} b_{J}$ leads to anyonic strings within the generalized definition, where anyonic means that mutual statistics of distinguishable 1D strings can go beyond bosonic or fermionic statistics. Both terms modify the quantum statistics of a string world sheet whose open ends host the 1D anyonic string. We can have another Aharonov-Bohm-like topological term with local one-form gauge field $a$ and three-form gauge field $c$, $\sim \int_{M^{5}} c \mathrm{~d} a$. When the above term appears together with other topological terms such as $\int_{M^{5}} a_{I} a_{J} a_{K} \mathrm{~d} a_{L}$, the statistics of $2 \mathrm{D}$ brane (attached to the end of three world volume) can have fractional statistics within the general definition, while the statistics of 0D particle (attached to the end of one world line $e^{i \int a}$ ) remains nonanyonic. Again the anyonic brane in $5 \mathrm{~d}$ can reduce to anyonic particles in $3 \mathrm{~d}$ by compactifying along $T^{2}$ in $M^{5}$ [18-20]. There are many other terms allowed in 5d [21]. For a general dimension $d$, there exists the topological term $\int c_{m} \mathrm{~d} c_{n} \sim \int c_{n} \mathrm{~d} c_{m}, n+m=d-1, n \leq m$, where $c_{n}$ is a $n$-form gauge field. We always take the higher-dimensional object from the $c_{m}$ field to have fractional statistics (the analogs of fractional flux), while we take the lower-dimensional object from the $c_{n}$ field to have regular statistics (the analogs of integrally quantized charge).

${ }^{7}$ We abbreviate both symmetry-protected topological state and symmetry-protected topological states as SPTs. 
we also call the generalized symmetric iTQFTs as higherSPTs. The boundary of higher-SPTs has higher-anomalies. Gauging the higher-symmetry of higher-SPTs gives rise to higher-gauge theories. Earlier pursuits on a systematic framework of the generalized iTQFTs through cobordism theories and cohomology theories include, but are not limited to, Refs. [44-53] and references therein. In this paper, we identify the $4 \mathrm{~d}$ anomalies of $\mathrm{SU}(\mathrm{N})_{\theta=\pi} \mathrm{YM}$ Eq. (1.2)-Eq. (1.4) with the mathematically precise $5 \mathrm{~d}$ bordism invariants, ${ }^{8}$ obtained in Refs. $[8,10]$.

Let us rephrase the higher anomalies into a condensed matter language on the lattice: Higher anomaly indicates that in order to realize all higher $n$-global symmetries locally on $n$-simplices, the theory needs to become a boundary of a onehigher dimensional higher-symmetry-protected topological state (higher-SPTs). If a theory has a higher anomaly, then the theory in its own dimension has some higher $n$-global symmetries acting non-locally (on $n$-simplices). Then there is an obstruction to gauge such a non-local symmetry, hence the name of higher ('t Hooft) anomaly [4]. More in Sec. 8.

\section{A. The Outline}

Here are the outlines of the present work:

(1) Section II.-We identify the $5 d$ bordism invariants with the 't Hooft anomalies of $4 \mathrm{~d} \mathrm{SU}(\mathrm{N})_{\theta=\pi} \mathrm{YM}$ theory (where we focus on $\mathrm{N}=2$ ), as a version of higher-anomaly matching.

(2) Section III.-We clarify and enumerate possible distinct classes of $4 \mathrm{~d} \mathrm{SU}(2)_{\theta=\pi} \mathrm{YM}$ theories. We take the condensed matter viewpoint, where we regard the $\mathrm{SU}(2)_{\theta=\pi} \mathrm{YM}$ theories as infrared theories emerging from high-energy ultraviolet (UV) bosonic systems with a lattice cutoff, as opposed to fermionic systems. We thus dub the UV system as bosonic YM theories. These bosonic YM theories still allow Wilson line operators as world lines of particles being (1) either bosonic or fermionic in quantum statistics, and (2) either Kramers doublet or Kramers singlet under the time-reversal symmetry. This supplements as a partial classification of $4 \mathrm{~d}$ $\mathrm{SU}(2)_{\theta=\pi}$ bosonic YM theories. We apply the tools in Ref. [38] to understand the relation between the

${ }^{8}$ For the mathematical terminology, we call the following:

(i) the bordism group generators as the manifolds or manifold generators, which generate finite Abelian groups, e.g., $\mathbb{Z}_{n}$.

(ii) the cobordism group generators as the topological terms or iTQFTs, which generate Abelian groups, e.g., $\mathbb{Z}_{n}$ or $\mathbb{Z}$, etc.

(iii) the (co)bordism invariants (people call bordism invariants as cobordism invariants with the same meaning) mean that they are invariants under the bordism class of manifolds, thus (co)bordism invariants mean the topological terms or iTQFTs, which again generate Abelian groups, $\mathbb{Z}_{n}$ or $\mathbb{Z}$, etc., gauge bundle constraint and the properties of line/ surface operators.

From Secs. II and III, we will see that there are at least four closely related $4 \mathrm{~d} \mathrm{SU}(2)_{\theta=\pi}$ nonsupersymmetric pure YM theories [which we nickname as four siblings of $4 \mathrm{~d} \mathrm{SU}(2)_{\theta=\pi}$ YM theories] with bosonic UV completions. Each of them carries either a distinct $4 \mathrm{~d}$ 't Hooft anomaly associated with $5 \mathrm{~d}$ higher-SPTs or a distinct $4 \mathrm{~d}$ counterterm. The distinct $5 \mathrm{~d}$ higherSPTs labeled by distinct $5 \mathrm{~d}$ bordism invariants are actually the physical analogs of the $5 \mathrm{~d}(4+1 \mathrm{D})$ oneform-center-symmetry-protected interacting topological superconductors in the condensed matter language.

(3) Sections IV and V.-We dynamically gauge the one-form center symmetry $\mathbb{Z}_{2,[1]}^{e}$. This turns the $4 d$ $\mathrm{SU}(2)_{\theta=\pi} \mathrm{YM} / 5 \mathrm{~d}$-higher-SPTs coupled systems in [8] into $4 \mathrm{~d} \mathrm{SO}(3)_{\theta=\pi} \mathrm{YM} / 5 \mathrm{~d}-$ higher-SETs coupled systems, where SETs stand for the symmetryenriched topologically ordered states. ${ }^{9}$ We then explore the detailed properties of various $5 \mathrm{~d}$ higher SETs. The $5 \mathrm{~d}$ higher SETs are described by $5 \mathrm{~d}$ timereversal symmetric higher-TQFTs with emergent two-form dynamical gauge fields. We mainly focus on the four siblings of $5 \mathrm{~d}$ higher SETs, while we also consider other highly relevant exotic $5 \mathrm{~d}$ higher SETs. To characterize these $5 \mathrm{~d}$ higher SETs, we study the following aspects:

(a) Partition function $\mathbf{Z}\left[M^{5}\right]$ without extended operator (one-line, two-surface, three-submanifold) insertions on five-manifold $M^{5}$. We compute $\mathbf{Z}\left[M^{5}\right]$ following the techniques and tools built from [12,13]. In particular, when $M^{5}=M^{4} \times S^{1}$, the partition function $\mathbf{Z}\left[M^{4} \times S^{1}\right]$ is the topological ground state degeneracy (topological GSD) on a spatial $M^{4}$. This issue is addressed in Sec. IV.

(b) Braiding statistics involving anyonic $1 \mathrm{D}$ string/2D branes and the associated link invariants of the spacetime two world sheet/three world volume. Here we compute the path integral $\mathbf{Z}\left[M^{5} ; W, U\right.$, ...] with extended-operator insertions $(W, U, \ldots)$, following the techniques and tools built from $[11,12,15,16]$. This issue is addressed in Sec. V.

(4) Section VI.-We provide the exemplary spacetime braiding processes of anyonic string/brane in $5 \mathrm{~d}$, and the link configurations of extended operators, which

\footnotetext{
${ }^{9}$ The SPTs is a short-ranged entangled quantum state that can be defined on a lattice. Once we break the global symmetry, SPTs can be deformed to a trivial product state under finite steps of local unitary transformations. SETs is a long-ranged entangled quantum state that can be defined on a lattice. (Here we only discuss the SETs that are anomaly-free.) Even if the global symmetry is completely broken, SETs cannot be deformed to a trivial product state under finite steps of local unitary transformations. The SETs have the same LRE nature as topologically ordered states. See recent reviews [29-32].
} 
TABLE I. A short summary of some results obtained in our work for the four sSiblings of $4 \mathrm{~d}$ pure nonsupersymmetric $\mathrm{SU}(2)_{\theta=\pi} \mathrm{YM}$ theories or SO(3) YM theories, and for the 4d-5d-SPT coupled systems or 4d-5d-higher-SET coupled systems.

Four Siblings of $5 d$ SRE-higher-SPTs-4d SU(2) $)_{\theta=\pi}$ YM coupled systems and their gauged analogous

Four Siblings of 5d LRE-higher-SETs-4d SO $(3)_{\theta=\pi}$ YM coupled systems

\begin{tabular}{c} 
(i). 5d higher-anomaly polynomial \\
(5d bordism invariants of $\left.\Omega_{5}^{\mathrm{O}}\left(\mathrm{B}^{2} \mathbb{Z}_{2}\right)\right)$ \\
involving 1-form center $\mathbb{Z}_{2,[1]}^{e}$ \\
time-reversal $\mathbb{Z}_{2}^{T}$-symmetries \\
\hline \hline d iTQFT / SPT partition function : \\
$\mathbf{Z}_{\mathrm{SPT}_{\left(K_{1}, K_{2}\right)}^{5 \mathrm{~d}}\left[M^{5}\right] .}$ \\
\hline \hline d TQFT / SET path integral : \\
$\mathbf{Z}_{\mathrm{SET}_{\left(K_{1}, K_{2}\right)}^{5 \mathrm{~d}}\left[M^{5}\right] .}$
\end{tabular}

(i). 5d higher-anomaly polynomial

(5d bordism invariants of $\left.\Omega_{5}^{\mathrm{O}}\left(\mathrm{B}^{2} \mathbb{Z}_{2}\right)\right)$

involving 1 -form center $\mathbb{Z}_{2}^{e}[1]$

time-reversal $\mathbb{Z}_{2}^{T}$-symmetries (ii). $4 \mathrm{~d} \mathrm{SU}(2)_{\theta=\pi} \mathrm{YM}$ obtained via dynamical gauging $4 \mathrm{~d}$ SPTs

(4d bordism invariants of $\Omega_{4}^{G^{\prime}}$ )

$G^{\prime}$ for a group extension:

$1 \rightarrow \mathrm{SU}(2) \rightarrow G^{\prime} \rightarrow \mathrm{O}(d) \rightarrow 1$.

Gauge and spacetime bundle/connection constraints

Wilson line operator $W$ properties (iii). 5d-spacetime-braiding process of anyonic-1D-strings/2D-branes

from 2-worldsheet and 3-worldvolume of 5d Higher-Gauge TQFTs/SETs:

Path-integral $\mathbf{Z}\left[M^{5}, \operatorname{Link}\right] / \mathbf{Z}\left[M^{5}\right]$ $\equiv\langle$ Link $\rangle$

New 5d Topological Link Invariants

$$
\begin{aligned}
& \text { 1st system }\left(K_{1}=0, K_{2}=0\right) \text { : } \\
& \frac{1}{2} \tilde{w}_{1}(T M) \mathcal{P}(B)=B \mathrm{Sq}^{1} B+\mathrm{Sq}^{2} \mathrm{Sq}^{1} B \\
& \sim w_{1} B B \\
& \text { Eq. (3.1) } \\
& G^{\prime}=\mathrm{O}(d) \times \mathrm{SU}(2) \text { in Eq. (3.5) }
\end{aligned}
$$

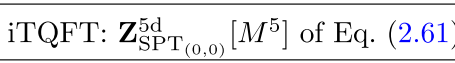

$$
\begin{aligned}
& \text { TQFT: } \mathbf{Z}_{\mathrm{SET}_{(0,0)}}^{5 \mathrm{~d}}\left[M^{5}\right] \text { of Eq. (4.1) } \\
& w_{2}\left(V_{\mathrm{PSU}(2)}\right)=B \\
& \text { Kramers singlet }\left(T^{2}=+1\right) \text { bosonic } W \\
& \text { 2nd system }\left(K_{1}=1, K_{2}=0\right) \text { : } \\
& \frac{1}{2} \tilde{w}_{1}(T M) \mathcal{P}(B)+w_{1}(T M)^{3} B \\
& =B \mathrm{Sq}^{1} B+w_{2}(T M) \mathrm{Sq}^{1} B \\
& \sim w_{1} B B+\left(w_{1}\right)^{3} B \\
& \text { Eq. (3.1) } \\
& G^{\prime}=\mathrm{E}(d) \times_{\mathbb{Z}_{2}} \mathrm{SU}(2) \text { in Eq. (3.7) } \\
& \text { iTQFT: } \mathbf{Z}_{\mathrm{SPT}_{(1,0)}^{5 \mathrm{~d}}\left[M^{5}\right] \text { of Eq. }(2.61)}
\end{aligned}
$$

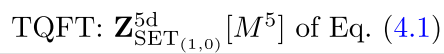

$$
\begin{aligned}
& \text { Eq. (5.96) } \\
& \begin{array}{c}
\hline \frac{1}{2} \#\left(V_{U_{h}}^{3} \cap \Sigma_{U_{b}}^{2}\right) \\
\equiv \frac{1}{2} \mathrm{Lk}_{w_{2} \mathrm{~d} B}^{(5)}\left(\Sigma_{U_{h}}^{2}, \Sigma_{U_{b}}^{2}\right), \\
\#\left(V_{U_{b}}^{3} \cap \Sigma_{U_{b}}^{2}\right) \\
\equiv \mathrm{Lk}_{B \mathrm{~d} B}^{(5)}\left(\Sigma_{U_{b}}^{2}, \Sigma_{U_{b}}^{2}\right)
\end{array}
\end{aligned}
$$

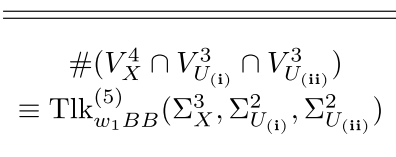

$\frac{\text { Eq. (3.1) }}{\frac{G^{\prime}=\mathrm{E}(d) \times_{\mathbb{Z}_{2}} \mathrm{SU}(2) \text { in Eq. (3.7) }}{w_{2}\left(V_{\mathrm{PSU}(2)}\right)=B+w_{1}(T M)^{2}}}$

3rd system $\left(K_{1}=0, K_{2}=1\right)$ :

$\frac{1}{2} \tilde{w}_{1}(T M) \mathcal{P}(B)+\mathrm{Sq}^{1}\left(w_{2}(T M) B\right)$ $\sim w_{1} B B+\frac{1}{2} \delta\left(w_{2} B\right)$

iTQFT: $\mathbf{Z}_{\mathrm{SPT}_{(0,1)}}^{5 \mathrm{~d}}\left[M^{5}\right]$ of Eq. (2.61)

TQFT: $\mathbf{Z}_{\mathrm{SET}_{(0,1)}}^{5 \mathrm{~d}}\left[M^{5}\right]$ of Eq. (4.1)
Eq. (3.1)

\begin{tabular}{c}
$\overline{G^{\prime}=\operatorname{Pin}^{+}(d) \times_{\mathbb{Z}_{2}} \mathrm{SU}(2) \text { in Eq. (3.10) }}$ \\
$w_{2}\left(V_{\mathrm{PSU}(2)}\right)=B+w_{2}(T M)$ \\
\hline
\end{tabular}

Kramers doublet $\left(T^{2}=-1\right)$ fermionic $W$

$$
\begin{aligned}
& \text { 4th system }\left(K_{1}=1, K_{2}=1\right) \text { : } \\
& \frac{1}{2} \tilde{w}_{1}(T M) \mathcal{P}(B)+w_{1}(T M)^{3} B+\mathrm{Sq}^{1}\left(w_{2}(T M) B\right) \\
& \sim w_{1} B B+\left(w_{1}\right)^{3} B+\frac{1}{2} \delta\left(w_{2} B\right) \\
& \text { or in a closed 5-manifold : } \\
& B \mathrm{Sq}^{1} B+w_{2}(T M) \mathrm{Sq}^{1} B \\
& \text { iTQFT: } \mathbf{Z}_{\mathrm{SPT}_{(1,1)}^{5 \mathrm{~d}}}\left[M^{5}\right] \text { of Eq. (2.61) } \\
& \text { TQFT: } \mathbf{Z}_{\mathrm{SET}_{(1,1)}}^{5 \mathrm{~d}}\left[M^{5}\right] \text { of Eq. (4.1) } \\
& \text { Eq. (5.96) } \\
& \begin{array}{c}
\frac{1}{2} \#\left(V_{U_{h}}^{3} \cap \Sigma_{U_{b}}^{2}\right) \\
\equiv \frac{1}{2} \mathrm{Lk}_{w_{2} \mathrm{~d} B}^{(5)}\left(\Sigma_{U_{h}}^{2}, \Sigma_{U_{b}}^{2}\right),
\end{array} \\
& \#\left(V_{U_{b}}^{3} \cap \Sigma_{U_{b}}^{2}\right) \\
& \equiv \mathrm{Lk}_{B \mathrm{~d} B}^{(5)}\left(\Sigma_{U_{b}}^{2}, \Sigma_{U_{b}}^{2}\right)
\end{aligned}
$$

\begin{tabular}{c}
\hline \hline$G^{\prime}=\operatorname{Pin}^{-}(d) \times_{\mathbb{Z}_{2}} \mathrm{SU}(2)$ in Eq. (3.12) \\
$w_{2}\left(V_{\mathrm{PSU}(2)}\right)=(B+$ \\
$\left.w_{1}(T M)^{2}+w_{2}(T M)\right)$ \\
\hline \hline
\end{tabular}

Eq. (5.25)

$\overline{\#\left(V_{X}^{4} \cap V_{U_{(\mathrm{i})}}^{3} \cap V_{U_{(\mathrm{ii})}}^{3}\right)}$
$\equiv \operatorname{Tlk}_{w_{1} B B}^{(5)}\left(\Sigma_{X}^{3}, \Sigma_{U_{(\mathrm{i})}}^{2}, \Sigma_{U_{(\mathrm{ii})}}^{2}\right)$

can be detected by the link invariants that we derived in Sec. V.

(5) Section VII.-We make more comments on the $4 \mathrm{~d}$ $\operatorname{SO}(3)_{\theta=\pi}$ YM theories, which live on the boundary of $5 \mathrm{~d}$ higher SETs. In particular, we reexamine these $4 \mathrm{~d} \mathrm{SO}(3)_{\theta=\pi}$ YM-5d-higher-SETs coupled systems in Sec. IV.
(6) Section VIII.-We construct the lattice regularization and UV completion of some of our systems. This includes a lattice realization of $5 \mathrm{~d}$ higher SPTs and higher-gauge SETs by implementing on a $5 \mathrm{~d}$ simplicial complex spacetime path integral and a $4+1 \mathrm{D}$ "condensed matter" realization on the spatial Hamiltonian operator. We also provide a lattice regularization 
of (1) higher-symmetry-extended and (2) highersymmetry-preserving anomalous $3+1 \mathrm{D}$ topologically ordered gapped boundaries by generalizing the method of [54]. The higher-symmetry-extension method was also developed in [55].

(7) Section IX.-We conclude and make connections to physics and mathematics in other perspectives.

Before we proceed to the detailed discussions in the main text, we first give a quick overview on more colloquial and pedestrian summaries in terms of schematic descriptions and Table I, in Sec. IB. Readers who are not familiar with certain mathematical information or physical motivations may seek for additional help from Refs. [8,10,38] (and its Appendixes).

\section{B. Summaries and Tables}

As we mentioned, in Secs. II and III, we will see that there are at least four closely related $4 \mathrm{~d} \mathrm{SU}(2)_{\theta=\pi}$ nonsupersymmetric pure YM theories [nicknamed the four siblings of $4 \mathrm{~d} \mathrm{SU}(2)_{\theta=\pi} \mathrm{YM}$ theories are labeled by $\left.\left(K_{1}, K_{2}\right) \in\left(\mathbb{Z}_{2}, \mathbb{Z}_{2}\right)\right]$ with a bosonic UV completion. They carry either distinct $4 \mathrm{~d}$ higher 't Hooft anomalies ${ }^{10}$ or distinct $4 \mathrm{~d}$ counterterms. All these anomalies that we will discuss below are the mod 2 nonperturbative global anomalies, similar to the old and the new SU(2) anomalies [56,57], except that instead of an ordinary global symmetry, now we require a higher 1 -form symmetry $\mathbb{Z}_{2,[1]}^{e}$ to probe higher anomalies. Here we advertise these results in a colloquial and pedestrian manner.

(1) $\left(K_{1}, K_{2}\right)=(0,0)$. The first sibling of $4 \mathrm{~d} \mathrm{SU}(2)_{\theta=\pi}$ with Kramers singlet $\left(T^{2}=+1\right)$ bosonic Wilson line has the $4 \mathrm{~d}$ anomaly/5d bordism invariant schematically as

$$
\sim w_{1}(T M) B B,
$$

with $w_{j}(T M)$ the $j$ th Stiefel-Whitney (SW) class of spacetime $M$ 's tangent bundle $T M$. Here $B \in$ $H^{2}\left(M, \mathbb{Z}_{2}\right)$ is a degree- 2 cohomology class obtained from restricting the two-form $\mathcal{B}$ field via $\mathcal{B} \sim \pi B$ and $\oint_{\Sigma} \mathcal{B}=\pi \mathbb{Z}$ for any closed surface $\Sigma$. More rigorously, $w_{1}(T M) B B$ stands for $\frac{1}{2} \tilde{w}_{1}(T M) \cup \mathcal{P}(B)$, explained in Sec. II.

(2) $\left(K_{1}, K_{2}\right)=(1,0)$. The second sibling of $4 \mathrm{~d}$ $\mathrm{SU}(2)_{\theta=\pi}$ with Kramers doublet $\left(T^{2}=-1\right)$ bosonic

\footnotetext{
${ }^{10}$ Distinct $4 \mathrm{~d}$ higher 't Hooft anomalies correspond to distinct $5 \mathrm{~d}$ higher-SPTs/counterterms labeled by distinct $5 \mathrm{~d}$ bordism invariants: physical analogs of $5 \mathrm{~d}(4+1 \mathrm{D})$ one-form-centersymmetry-protected interacting "topological superconductors" in a condensed matter language. In condensed matter, topological superconductors refer to electronic systems with time-reversal symmetry but without $\mathrm{U}(1)$ electron charge conservation symmetry (see an overview [30,31]), for example, due to the Cooper pairing breaking $\mathrm{U}(1)$ down to a discrete subgroup or down to nothing.
}

Wilson line has the $4 \mathrm{~d}$ anomaly/5d bordism invariant schematically as

$$
\sim w_{1}(T M) B B+w_{1}(T M)^{3} B .
$$

We note that the $4 \mathrm{~d}$ anomaly associated with the $5 \mathrm{~d}$ $w_{1}(T M)^{3} B$ term is highly related to the $2 \mathrm{~d}$ charge conjugation anomaly associated with the $3 \mathrm{~d}$ cubic $A^{3}$ term for a $\mathbb{Z}_{2}$-valued one-cohomology class $A$. See the relevant studies of the $2 \mathrm{~d}$ anomaly from the $3 \mathrm{~d}$ cubic $A^{3}$ term in $[8,22,58-60]$ and references therein.

(3) $\left(K_{1}, K_{2}\right)=(0,1)$. The third sibling of $4 \mathrm{~d} \mathrm{SU}(2)_{\theta=\pi}$ with Kramers doublet $\left(T^{2}=-1\right)$ fermionic Wilson line has the $4 \mathrm{~d}$ anomaly $/ 5 \mathrm{~d}$ bordism invariant schematically as

$$
\sim w_{1}(T M) B B+\frac{1}{2} \delta\left(w_{2}(T M) B\right) .
$$

Here $\delta$ is a coboundary operator, sending a $j$-cochain in the cochain group $C^{j}\left(M, \mathbb{Z}_{n}\right)$ to a $(j+1)$ coboundary in the coboundary group $B^{j+1}\left(M, \mathbb{Z}_{n}\right)$. Note that there are maps $M \rightarrow \mathrm{BO}$ and $M \rightarrow \mathrm{B}^{2} \mathbb{Z}_{2}$, so $w_{2}(T M) B$ in the cohomology group $H^{4}(\mathrm{BO} \times$ $\mathrm{B}^{2} \mathbb{Z}_{2}, \mathbb{Z}_{2}$ ) can be pulled back to another cohomology group $H^{4}\left(M, \mathbb{Z}_{2}\right)$, with $\mathrm{O}$ the orthogonal group $\mathrm{O}(d)$ for the $d$ manifold. In this case, the $w_{2}(T M) B$ is a cohomology class in $H^{4}\left(M, \mathbb{Z}_{2}\right)$. Meanwhile $\frac{1}{2} \delta\left(w_{2}(T M) B\right)$ sends $w_{2}(T M) B$ to a cohomology class in $H^{5}\left(M, \mathbb{Z}_{2}\right)$. The $\frac{1}{2} \delta$ is mathematically precisely a Steenrod square $\mathrm{Sq}^{1}$ [61].

(4) $\left(K_{1}, K_{2}\right)=(1,1)$. The fourth sibling of $4 \mathrm{~d} \mathrm{SU}(2)_{\theta=\pi}$ with Kramers singlet $\left(T^{2}=+1\right)$ fermionic Wilson line has the $4 \mathrm{~d}$ anomaly $/ 5 \mathrm{~d}$ bordism invariant schematically as

$$
\sim w_{1}(T M) B B+w_{1}(T M)^{3} B+\frac{1}{2} \delta\left(w_{2}(T M) B\right) .
$$

We remark that our investigations on Kramers timereversal properties and bosonic/fermionic statistics of line operators (for non-Abelian gauge theories here) give rise to a further refined classification of gauge theories somehow beyond the previous framework of Refs. [6,62]. See Refs. $[63,64]$ for the case of Abelian U(1) gauge theories. See also $[38,65,66]$ for other examples of non-Abelian gauge theories.

The schematic $\int_{M^{5}} \frac{1}{2} \delta\left(w_{2}(T M) B\right)$ term in Eq. (1.7) and Eq. (1.8) is written as mathematically precisely $\int_{M^{5}} \mathrm{Sq}^{1}\left(w_{2}(T M) B\right)$ on a five manifold $M^{5}$ in Sec. II. We will see that such a term $\int_{M^{5}} \mathrm{Sq}^{1}\left(w_{2}(T M) B\right)$ vanishes (as the $0 \bmod 2$ ), when $M^{5}$ is a closed five manifold. However, Sec. II shows that when $M^{5}$ has a boundary $M^{4}=\partial M^{5}, \quad \int_{M^{5}} \mathrm{Sq}^{1}\left(w_{2}(T M) B\right)$ transforms nontrivially under $B \rightarrow B+\delta \lambda$ where $\lambda$ is a one-cochain. This nontrivial 


\section{(a)}

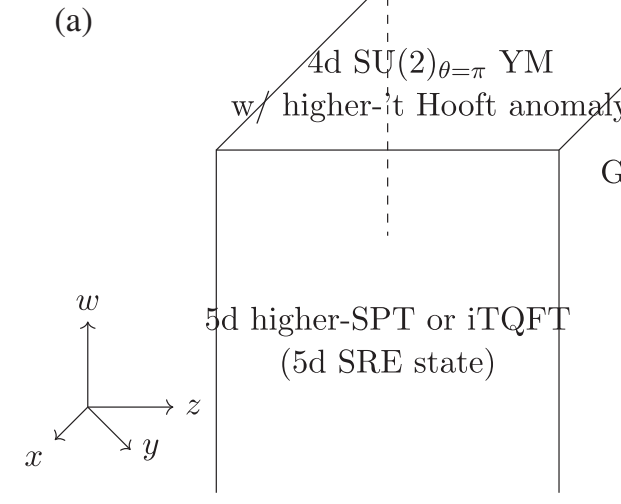

(b)

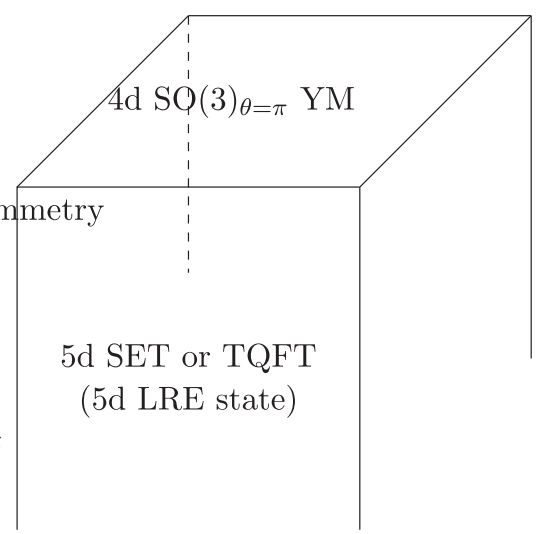

FIG. 1. (a) Schematic illustration of $4 d-5 d$ coupled system: $\mathrm{SU}(2)_{\theta=\pi}$ YM-5d SRE higher-SPTs coupled systems. There are four siblings of such systems with bosonic UV completion, summarized in Table I. We use $x, y, z$ to label the spatial coordinates of $4 \mathrm{~d}$ ( $3+1 \mathrm{D}) \mathrm{YM}$, and we introduce an extra coordinate $w$ to label the additional dimension of $5 \mathrm{~d}$ higher SPTs. (b) Schematic illustration of $4 \mathrm{~d}-5 \mathrm{~d}$ coupled system: $4 \mathrm{~d} \mathrm{SO}(3)_{\theta=\pi}$ YM-5d LRE higher-SETs coupled systems via gauging one-form $\mathbb{Z}_{2,[1]}^{e}$ center symmetry in Fig. 1(a). There are four siblings of such 5d SET systems with bosonic UV completion, summarized in Table I. We use $x, y, z$ to label the spatial coordinates of 4d (3 + 1D) YM, and we introduce an extra coordinate $w$ to label the additional dimension of 5d higher SETs. See also Fig. 16.

transformation is essential to cancel the noninvariance of the $4 \mathrm{~d}$ YM theory. This observation indicates a subtle fact that $\int_{M^{5}} \operatorname{Sq}^{1}\left(w_{2}(T M) B\right)$ cannot be dropped and should be kept as a certain physical term, since we are studying the physics on a $5 \mathrm{~d}$ manifold with a $4 \mathrm{~d}$ boundary. To summarize:

(i) $\int_{M^{5}} \mathrm{Sq}^{1}\left(w_{2}(T M) B\right)$ vanishes as $0(\bmod 2)$ on a closed five manifold $M^{5}$. This can be interpreted in many distinct but related ways. It describes a trivial gapped vacuum with no SPT order, or a trivial gapped insulator in condensed matter language, or a trivial iTQFT on $M^{5}$.

(ii) However, $\int_{M^{5}} \mathrm{Sq}^{1}\left(w_{2}(T M) B\right)$ has essential physical effects on a five manifold $M^{5}$ with a nontrivial boundary $M^{4}=\partial M^{5}$. Under the background gauge transformation $B \rightarrow B+\delta \lambda$, the gauge variant is nonzero.

(iii) $\int_{M^{5}} \operatorname{Sq}^{1}\left(w_{2}(T M) B\right)=\int_{M^{5}} \frac{1}{2} \delta\left(w_{2}(T M) B\right)$ on an $M^{5}$ with boundary $M^{4}=\partial M^{5}$ may behave as $\int_{M^{4}} \frac{1}{2}\left(w_{2}(T M) B\right)$ - which is half of a $4 \mathrm{~d}$ bordism invariant $w_{2}(T M) B$. Twice of this fractional term $\sim 2 \int_{M^{4}} \frac{1}{2}\left(w_{2}(T M) B\right) \sim \int_{M^{4}}\left(w_{2}(T M) B\right)$ is a $4 \mathrm{~d}$ bordism invariant, and the quadruple of this fractional term $4 \int_{M^{4}} \frac{1}{2}\left(w_{2}(T M) B\right) \sim 2 \int_{M^{4}}\left(w_{2}(T M) B\right)=0 \bmod 2$ is a trivial $4 \mathrm{~d}$ bordism invariant. Thus $\int_{M^{5}} \mathrm{Sq}^{1} \times$ $\left(w_{2}(T M) B\right)$ cannot be interpreted as a $4 \mathrm{~d}$ local counterterm. Instead, we interpret it as a nonlocal counterterm or a fractional counterterm on $M^{4}$. This is analogous to a certain Wess-Zumino-Witten (WZW)like term $^{11}$ with the following new features:

\footnotetext{
${ }^{11}$ We thank Ho Tat Lam for an inspiring conversation on this issue.
}

(i) The standard WZW term $[67,68]$ is labeled by an integer, but here $\int_{M^{5}} \operatorname{Sq}^{1}\left(w_{2}(T M) B\right)$ is labeled by a $\mathbb{Z}_{2}$ number.

(ii) The standard WZW term is written in terms of dynamical fields, but the WZW-like term here is written in terms of the background fields of the time-reversal symmetry $\mathbb{Z}_{2}^{T}$ and a higher symmetry $\mathbb{Z}_{2,[1]}^{e}$.

(iv) Similar to the standard WZW term, our WZWlike term affects the symmetry quantum numbers of physical observables, i.e., the statistics and Kramers degeneracy (i.e., singlet or doublet) of the Wilson lines.

A schematic illustration of $4 \mathrm{~d} \mathrm{SU}(2)_{\theta=\pi}$ YM-5d SRE higher-SPTs coupled system is shown in Fig. 1. See Table I for a short summary for the four siblings of $4 \mathrm{~d}$ $\mathrm{SU}(2)_{\theta=\pi}$ YM theories and their coupling to the $5 \mathrm{~d}$ systems, as well as their physical properties. See Table II for a summary of the link invariants and link configurations of $5 \mathrm{~d}$ TQFTs.

\section{4D SU(2) $\theta=\pi$ YANG-MILLS GAUGE THEORIES COUPLED TO 5D SHORT-RANGE ENTANGLED SPTS}

\section{A. Ordinary and higher global symmetries of Yang-Mills theory}

We discuss the global symmetries of $\mathrm{SU}(\mathrm{N})_{\theta} \mathrm{YM}$ theory in Eq. (1.1).

(1) We first focus on the discrete time-reversal symmetry $\mathbb{Z}_{2}^{T}$ and its symmetry transformation $\mathcal{T}$ acting on the gauge field $a_{\mu} \equiv a_{\mu}^{\alpha} T^{\alpha}$, where the temporal component is $a_{0}$ and the spatial component is $a_{i} . \mathcal{T}$ acts on $a_{\mu}$ as 
TABLE II. Link invariants and link configurations of two world sheet and three world volume from the anyonic-1D-strings/2Dbranes' spacetime braiding processes in 5d higher-gauge time-reversal SETs in Secs. V and VI. Readers can find other related link invariants in $3 \mathrm{~d}, 4 \mathrm{~d}$, and others in the tables of $[11,12]$.

Section and link invariant

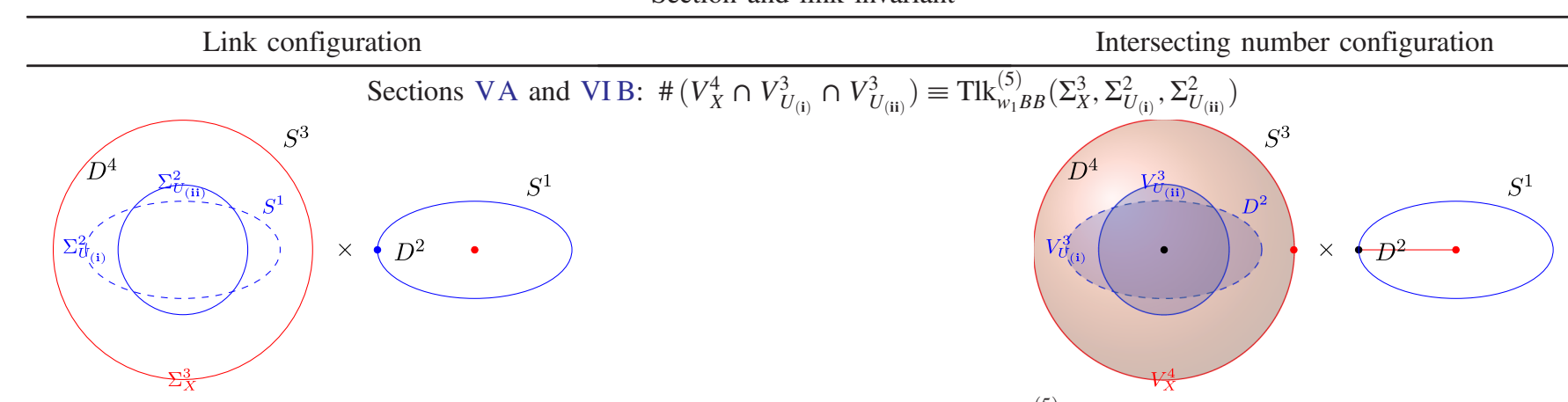

Sections V B 2, V D, and VIC: \# $\left(V_{X_{(\mathbf{i})}}^{4} \cap V_{X_{(i i)}}^{4} \cap \Sigma_{U}^{2}\right) \equiv \mathrm{Tlk}_{w_{1} w_{1} \mathrm{~d} B}^{(5)}\left(\Sigma_{X_{(\mathbf{i})}}^{3}, \Sigma_{X_{(\mathbf{i i})}}^{3}, \Sigma_{U}^{2}\right)$
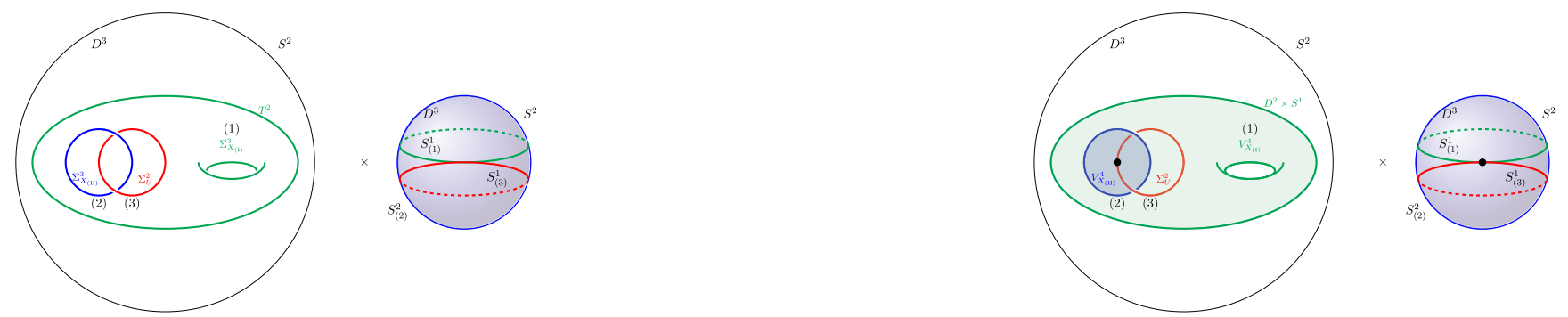

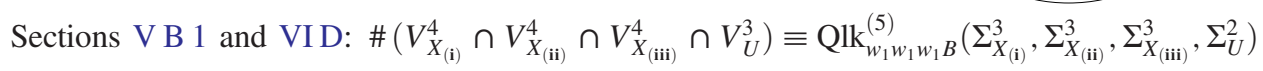
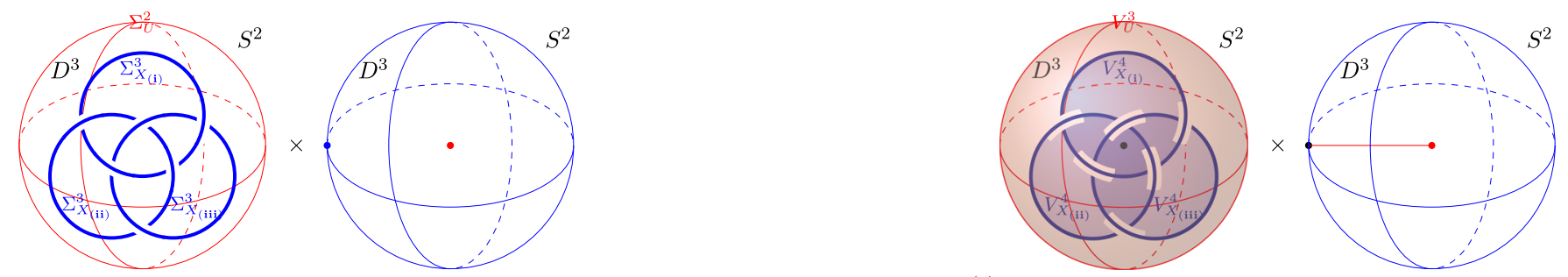

Sections VD and VIE: \# $\left(V_{U_{(\mathrm{i})}}^{3} \cap \Sigma_{U_{(i \mathrm{i})}}^{2}\right) \equiv \mathrm{Lk}_{B \mathrm{~d} B}^{(5)}\left(\Sigma_{U_{(\mathrm{i})}}^{2}, \Sigma_{U_{(\mathrm{ii})}}^{2}\right)$
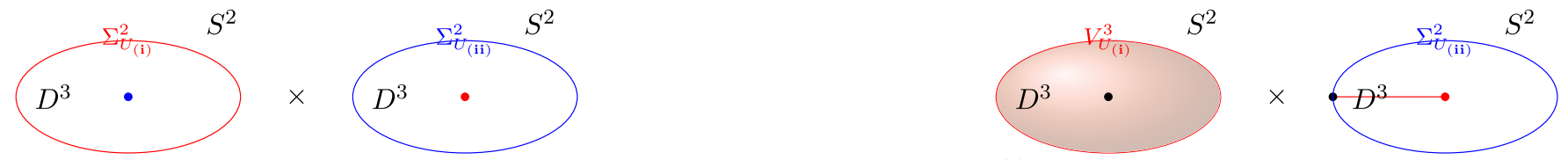

Sections V C, VD, and VIF: \# $\left(V_{U^{\prime}}^{3} \cap \Sigma_{U}^{2}\right) \equiv \mathrm{Lk}_{w_{2} \mathrm{~d} B}^{(5)}\left(\Sigma_{U}^{2}, \Sigma_{U^{\prime}}^{2}\right)$
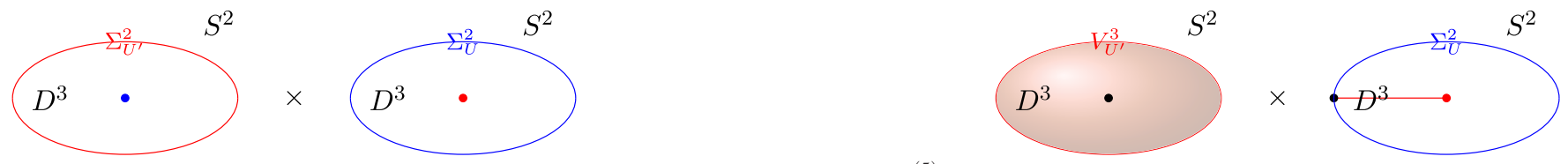

Section VI G: \# $\left(V_{X_{(\mathrm{i})}}^{4} \cap \Sigma_{X_{(i \mathrm{i})}}^{3} \cap V_{U}^{3}\right) \equiv \operatorname{Tlk}_{(A \mathrm{~d} A) B}^{(5)}\left(\Sigma_{X_{(\mathrm{i})}}^{3}, \Sigma_{X_{(\mathrm{ii})}}^{3}, \Sigma_{U}^{2}\right)$
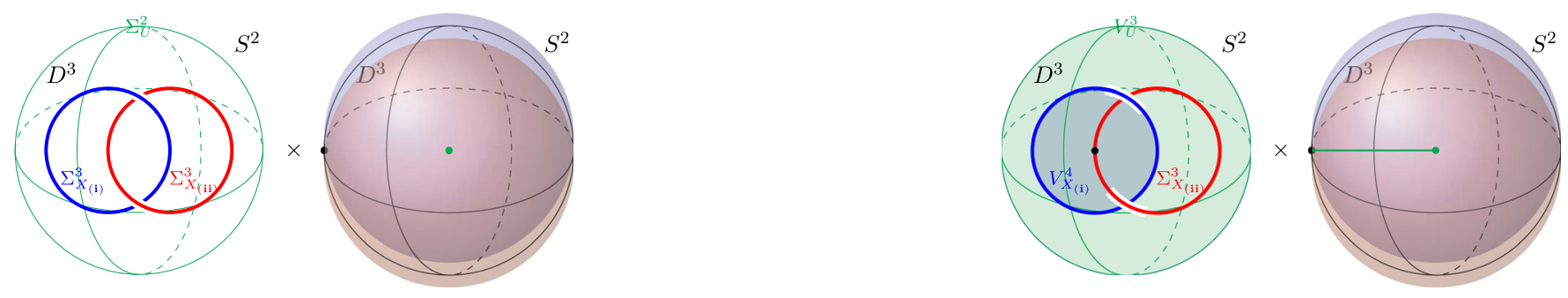


$$
\begin{aligned}
& \mathcal{T}: a_{0}^{\alpha} \rightarrow-a_{0}^{\alpha}, \quad a_{i}^{\alpha} \rightarrow a_{i}^{\alpha}, \quad\left(t, x_{i}\right) \rightarrow\left(-t, x_{i}\right), \\
& T^{\alpha} \rightarrow T^{\alpha}, \quad a_{0} \rightarrow-a_{0}, \quad a_{i} \rightarrow a_{i} .
\end{aligned}
$$

The components of the field strength are $F_{i j}=F_{i j}^{\alpha} T^{\alpha}$ and $F_{0 i}=F_{0 i}^{\alpha} T^{\alpha}$. Under $\mathcal{T}, F_{i j}^{\alpha}$ is preserved, while $F_{0 i}^{\alpha}$ flips sign.

$$
\begin{aligned}
\mathcal{T}: F_{i j}^{\alpha} & =\partial_{i} a_{j}^{\alpha}-\partial_{j} a_{i}^{\alpha}+f^{\alpha \beta \gamma} a_{i}^{\beta} a_{j}^{\gamma} \rightarrow \partial_{i} a_{j}^{\alpha}-\partial_{j} a_{i}^{\alpha}+f^{\alpha \beta \gamma} a_{i}^{\beta} a_{j}^{\gamma}=F_{i j}^{\alpha}\left(-t, x_{i}\right), \\
F_{0 i}^{\alpha} & =\partial_{t} a_{i}^{\alpha}-\partial_{i} a_{0}^{\alpha}+f^{\alpha \beta \gamma} a_{0}^{\beta} a_{i}^{\gamma} \rightarrow-\partial_{-t} a_{i}^{\alpha}+\partial_{i} a_{0}^{\alpha}-f^{\alpha \beta \gamma} a_{0}^{\beta} a_{i}^{\gamma}=-F_{0 i}^{\alpha}\left(-t, x_{i}\right) .
\end{aligned}
$$

Here $f^{\alpha \beta \gamma}$ is the structure constant of the $\mathrm{SU}(\mathrm{N})$ Lie algebra which is real. The reason that this $\mathcal{T}$ is a good symmetry choice in contrast to the familiar $\mathcal{T}$ symmetry of the $\mathrm{U}(1)$ gauge theory case is explained in the footnote. ${ }^{12}$ It is obvious that the kinetic term $\int_{M^{4}} \operatorname{Tr}(F \wedge \star F)$ is invariant under $\mathcal{T}$. The $\theta$ term flips the sign under $\mathcal{T}^{13}$ :

$$
\mathcal{T}: \frac{\theta}{8 \pi^{2}} \int_{M^{4}} \operatorname{Tr}(F \wedge F) \rightarrow-\frac{\theta}{8 \pi^{2}} \int_{M^{4}} \operatorname{Tr}(F \wedge F)
$$

The $\theta \in[0,2 \pi)$ has a $2 \pi$ periodicity, and thus the theories at $\theta=0$ and $\theta=\pi$ are time-reversal invariant.

(2) We can define the $\mathbb{Z}_{2}^{C T}$ symmetry associated with the $\mathcal{C} \mathcal{T}$ transformation for an $\mathrm{SU}(\mathrm{N})$ gauge theory:

$$
\begin{aligned}
\mathcal{C} \mathcal{T}: a_{0}^{\alpha} & \rightarrow-a_{0}^{\alpha}, \quad a_{i}^{\alpha} \rightarrow+a_{i}^{\alpha}, \quad\left(t, x_{i}\right) \rightarrow\left(-t, x_{i}\right), \\
T^{\alpha} & \rightarrow-T^{\alpha *}, \quad a_{0} \rightarrow+a_{0}^{*}, \quad a_{i} \rightarrow-a_{i}^{*}, \\
F_{0 i}^{\alpha} & \rightarrow-F_{0 i}^{\alpha}\left(-t, x_{i}\right), \quad F_{0 i}=F_{0 i}^{\alpha} T^{\alpha} \rightarrow\left(-F_{0 i}^{\alpha}\left(-t, x_{i}\right)\right)\left(-T^{\alpha *}\right)=F_{0 i}^{*}\left(-t, x_{i}\right), \\
F_{i j}^{\alpha} & \rightarrow F_{i j}^{\alpha}\left(-t, x_{i}\right), \quad F_{i j}=F_{i j}^{\alpha} T^{\alpha} \rightarrow F_{i j}^{\alpha}\left(-t, x_{i}\right)\left(-T^{\alpha *}\right)=-F_{i j}^{*}\left(-t, x_{i}\right) .
\end{aligned}
$$

Here $*$ is the complex conjugation. We also define the charge conjugation $\mathbb{Z}_{2}^{C}$ symmetry associated with the $\mathcal{C}$ transformation for an $\mathrm{SU}(\mathrm{N})$ gauge theory:

\footnotetext{
${ }^{12}$ The familiar U(1) gauge theory sends $a_{0} \rightarrow a_{0}$ and $a_{i} \rightarrow-a_{i}$. If we choose instead $a_{0}^{\alpha} \rightarrow a_{0}^{\alpha}$ and $a_{i}^{\alpha} \rightarrow-a_{i}^{\alpha}$ for SU(N) gauge field, then $F_{i j}^{\alpha}$ and $F_{0 i}^{\alpha}$ are not mapped back to themselves (not even up to a sign); thus this does not define any symmetry of SU(N) YM. Given a gauge group $G$, the above discussion is related to the center $\mathrm{Z}(G)$, the automorphism group $\operatorname{Aut}(G)$, the outer automorphism Out $(G)$, and the inner automorphism $\operatorname{Inn}(G)$. They form short exact sequences:

$$
1 \rightarrow \mathrm{Z}(G) \rightarrow G \rightarrow \operatorname{Inn}(G) \rightarrow 1, \quad \text { and } \quad 1 \rightarrow \operatorname{Inn}(G) \rightarrow \operatorname{Aut}(G) \rightarrow \operatorname{Out}(G) \rightarrow 1,
$$
}

and a combined exact sequence

$$
1 \rightarrow \mathrm{Z}(G) \rightarrow G \rightarrow \operatorname{Aut}(G) \rightarrow \operatorname{Out}(G) \rightarrow 1 .
$$

If $G$ is a simply connected compact Lie group and $\mathbf{g}$ is its Lie algebra (which would necessarily be semisimple), then $\operatorname{Inn}(G)=\operatorname{Inn}(\mathbf{g})=\mathrm{P} G, \operatorname{Aut}(G)=\operatorname{Aut}(\mathbf{g})$, and $\operatorname{Out}(G)=\operatorname{Out}(\mathbf{g})=\operatorname{Aut}\left(D_{\mathbf{g}}\right)$ is isomorphic to the automorphism group of the Dynkin diagram $D_{\mathbf{g}}$ of the Lie algebra $\mathbf{g}$.

(i) For $G=\mathrm{U}(1)$, we have $\mathrm{Z}(G)=\mathrm{U}(1), \operatorname{Inn}(G)=1, \operatorname{Aut}(G)=\operatorname{Out}(G)=\mathbb{Z}_{2}$.

(ii) For $G=\mathrm{SU}(2)$, we have $\mathrm{Z}(G)=\mathbb{Z}_{2}, \operatorname{Inn}(G)=\operatorname{SO}(3)$, $\operatorname{Aut}(G)=\operatorname{PSU}(2)=\operatorname{SO}(3)$, and $\operatorname{Out}(G)=1$.

(iii) For $G=\mathrm{SU}(\mathrm{N})$ with $\mathrm{N} \geq 3$, we have $\mathrm{Z}(G)=\mathbb{Z}_{\mathrm{N}}, \operatorname{Inn}(G)=\operatorname{PSU}(\mathrm{N})$, and Out $(G)=\mathbb{Z}_{2}$. We also have Aut $(G)=\operatorname{PSU}(\mathrm{N}) \rtimes \mathbb{Z}_{2}$ where $\mathbb{Z}_{2}$ acts on PSU(N) by Eq. (2.3)'s $T^{\alpha} \rightarrow-T^{\alpha *}$ and $a \rightarrow-a^{*}$ with a minus sign and a complex conjugation.

The validity of the charge conjugation symmetry $\mathbb{Z}_{2}^{C}$, with a $\mathcal{C}$ global symmetry transformation, is based on the validity of the outer automorphism $\operatorname{Out}(G)$ that includes a $\mathbb{Z}_{2}$ as a $\mathbb{Z}_{2}^{C}$.

${ }^{13}$ More explicitly, under $\mathcal{T}$ [using Eq. (2.2)]

$$
\mathcal{T}: \epsilon^{i j k} F_{0 i}^{\alpha}(t, x) F_{j k}^{\alpha}(t, x) \rightarrow-\epsilon^{i j k} F_{0 i}^{\alpha}(-t, x) F_{j k}^{\alpha}(-t, x) .
$$

The time reversal changes the sign in the bracket of the field $f(t) \rightarrow f(-t)$, and the integration measure maintains $\mathrm{d} t \rightarrow \mathrm{d} t$. 


$$
\begin{aligned}
\mathcal{C}: a_{0}^{\alpha} & \rightarrow a_{0}^{\alpha}, \quad a_{i}^{\alpha} \rightarrow a_{i}^{\alpha}, \quad\left(t, x_{i}\right) \rightarrow\left(t, x_{i}\right), \\
T^{\alpha} & \rightarrow-T^{\alpha *}, \quad a_{0} \rightarrow-a_{0}^{*}, \quad a_{i} \rightarrow-a_{i}^{*}, \\
F_{0 i} & =F_{0 i}^{\alpha} T^{\alpha} \rightarrow-F_{0 i}^{*}, \\
F_{i j} & =F_{i j}^{\alpha} T^{\alpha} \rightarrow-F_{i j}^{*} .
\end{aligned}
$$

However, for $\mathrm{N}=2$, the $\mathrm{SU}(2) \mathrm{YM}$ does not have $\mathbb{Z}_{2}^{C}$ global symmetry because $\mathrm{SU}(2)$ does not have a $\mathbb{Z}_{2}$ outer automorphism. The $\mathcal{C}$ transformation is part of the $\mathrm{SU}(2)$ gauge transformation. Let $\mathcal{C}_{\mathrm{SU}(2)}=e^{\mathrm{i} \frac{\pi}{2} \sigma_{2}} \in \mathrm{SU}(2)$ be the matrix that provides an isomorphism between the fundamental representation of $\mathrm{SU}(2)$ and its conjugate, and let $U_{\mathrm{SU}(2)}=\exp \left(\mathrm{i} \frac{\theta}{2} \sigma_{\alpha}\right)$ be the unitary $\mathrm{SU}(2)$ transformation on the $\mathrm{SU}(2)$ fundamentals, where $\sigma_{\alpha}, \alpha=1,2,3$ are Pauli matrices. Then $\mathcal{C}_{\mathrm{SU}(2)} U_{\mathrm{SU}(2)} \mathcal{C}_{\mathrm{SU}(2)}^{-1}=\exp \left(-\mathrm{i} \frac{\theta}{2} \sigma_{\alpha}^{T}\right)=\exp \left(-\mathrm{i} \frac{\theta}{2} \sigma_{\alpha}^{*}\right)=U_{\mathrm{SU}(2)}^{*}$. In other words, $\mathbb{Z}_{2}^{T}$ and $\mathbb{Z}_{2}^{C T}$ are the same symmetry for the SU(2) YM. See more discussions in footnote 11, Sec. 2.2 of [38], and Sec. 2 of [8].

(3) Parity symmetry $\mathbb{Z}_{2}^{P}$ is another discrete symmetry associated with the transformation $\mathcal{P}$ :

$$
\begin{aligned}
\mathcal{P}: a_{0}^{\alpha} & \rightarrow a_{0}^{\alpha}, \quad a_{i}^{\alpha} \rightarrow-a_{i}^{\alpha}, \quad\left(t, x_{i}\right) \rightarrow\left(t,-x_{i}\right), \\
T^{\alpha} & \rightarrow T^{\alpha}, \quad a_{0} \rightarrow a_{0}, \quad a_{i} \rightarrow-a_{i}, \\
F_{i j}^{\alpha} & =\partial_{i} a_{j}^{\alpha}-\partial_{j} a_{i}^{\alpha}+f^{\alpha \beta \gamma} a_{i}^{\beta} a_{j}^{\gamma} \rightarrow \partial_{-i}\left(-a_{j}^{\alpha}\right)-\partial_{-j}\left(-a_{i}^{\alpha}\right)+f^{\alpha \beta \gamma}\left(-a_{i}^{\beta}\right)\left(-a_{j}^{\gamma}\right)=F_{i j}^{\alpha}\left(-t, x_{i}\right), \\
F_{0 i}^{\alpha} & =\partial_{t} a_{i}^{\alpha}-\partial_{i} a_{0}^{\alpha}+f^{\alpha \beta \gamma} a_{0}^{\beta} a_{i}^{\gamma} \rightarrow \partial_{t}\left(-a_{i}^{\alpha}\right)+\partial_{-i} a_{0}^{\alpha}+f^{\alpha \beta \gamma} a_{0}^{\beta}\left(-a_{i}^{\gamma}\right)=-F_{0 i}^{\alpha}\left(t,-x_{i}\right) .
\end{aligned}
$$

$\mathcal{P}$ is related to $\mathcal{C} \mathcal{T}$ via the $\mathcal{C P} \mathcal{T}$ symmetry:

$$
\begin{aligned}
\mathcal{C P} \mathcal{T}: a_{0}^{\alpha} & \rightarrow-a_{0}^{\alpha}, \quad a_{i}^{\alpha} \rightarrow-a_{i}^{\alpha}, \quad\left(t, x_{i}\right) \rightarrow\left(-t,-x_{i}\right), \\
T^{\alpha} & \rightarrow-T^{\alpha *}, \quad a_{\mu} \rightarrow+a_{\mu}^{*}, \\
F_{0 i}^{\alpha} & \rightarrow F_{0 i}^{\alpha}\left(-t,-x_{i}\right), \quad F_{0 i}=F_{0 i}^{\alpha} T^{\alpha} \rightarrow F_{0 i}^{\alpha}\left(-t,-x_{i}\right)\left(-T^{\alpha *}\right)=-F_{0 i}^{*}\left(-t,-x_{i}\right), \\
F_{i j}^{\alpha} & \rightarrow F_{i j}^{\alpha}\left(-t,-x_{i}\right), \quad F_{i j}=F_{i j}^{\alpha} T^{\alpha} \rightarrow F_{i j}^{\alpha}\left(-t,-x_{i}\right)\left(-T^{\alpha *}\right)=-F_{i j}^{*}\left(-t,-x_{i}\right) .
\end{aligned}
$$

(4) The one-form electric $\mathbb{Z}_{\mathrm{N},[1]}^{e}$ center global symmetry: The charged object of the one-form $\mathbb{Z}_{\mathrm{N},[1]}^{e}$ symmetry is a gauge-invariant Wilson line

$$
W_{e}^{\mathrm{R}}=\operatorname{Tr}_{\mathrm{R}}(\mathrm{P} \exp (\mathrm{i} \oint a)) .
$$

The gauge field $a$ is Lie algebra su(N) valued. The $\mathrm{P} \exp (\mathrm{i} \oint a)$ specifies a $\mathrm{SU}(\mathrm{N})$ group element where $\mathrm{P}$ is the path ordering. $\mathrm{Tr}$ is the trace in the representation $\mathrm{R}$ of $\mathrm{SU}(\mathrm{N})$. For the $\mathrm{SU}(\mathrm{N})$ gauge theory, $\mathrm{R}$ can be any possible representation. If $\mathrm{R}$ is an irreducible representation and $l$ is the number of boxes in the Young diagram of $\mathrm{R}$, then $W_{e}$ transforms under $\mathbb{Z}_{\mathrm{N},[1]}^{e}$ as

$$
\mathbb{Z}_{\mathrm{N},[1]}^{e}: W_{e}^{\mathrm{R}} \rightarrow e^{2 \pi i l / \mathrm{N}} W_{e}^{\mathrm{R}} .
$$

For the fundamental representation, there is only one box in the Young diagram; hence the Wilson line $W_{e}^{\text {fund }}$ transforms under $\mathbb{Z}_{\mathrm{N},[1]}^{e}$ as $W_{e}^{\text {fund }} \rightarrow e^{2 \pi i / \mathrm{N}} W_{e}^{\text {fund }}$. For $\mathrm{N}=2$, the Wilson line in the fundamental representation transforms under $\mathbb{Z}_{2,[1]}^{e}$ by a sign $W_{e}^{\text {fund }} \rightarrow-W_{e}^{\text {fund }}$.

The charge operator (i.e., symmetry generator) of the $\mathbb{Z}_{\mathrm{N},[1]}^{e}$ symmetry is a codimension 2 (thus a $2 \mathrm{D}$ operator in $4 \mathrm{~d}$ spacetime) electric surface operator $U_{e}$. For SU(2) gauge theory, we will see that

$$
U_{e}=\exp (\mathrm{i} \pi \oint \Lambda)
$$

where $\Lambda \in H^{2}\left(M^{4}, \mathbb{Z}_{2}\right)$ as a cohomology class.

One can couple the $\mathrm{SU}(2)$ theory to $\mathbb{Z}_{\mathrm{N},[1]}^{e}$ background gauge field $B$. Following [5,6,69], we first promote the $\mathrm{SU}(2)$ gauge field $a$ to a $\mathrm{U}(2)$ gauge field $\hat{a}$,

$$
\hat{a}=a+\frac{1}{2} \hat{A}_{2},
$$

where $\mathbb{I}_{2}$ is a two-dimensional identity matrix. The first Chern class of the $\mathrm{U}(2)$ bundle is $c_{1} \equiv$ $c_{1}\left(V_{\mathrm{U}(2)}\right) \equiv \frac{\operatorname{Tr} \hat{F}}{2 \pi} \equiv \frac{\mathrm{d} \hat{A}}{2 \pi}$ where $\hat{F}=\mathrm{d} \hat{a}-\mathrm{i} \hat{a} \wedge \hat{a}$ is a $\mathrm{U}$ (2) field strength. Then we couple to $B$ by requiring 
$c_{1}=B \bmod 2$, which can be done via introducing a Lagrangian multiplier $\Lambda$ [see Eq. (2.37)]. This amounts to introducing the following term in the path integral:

$$
\int[\mathcal{D} \Lambda] \cdots \exp \left(\mathrm{i} \pi \int_{M^{4}} \Lambda \cup\left(c_{1}-B\right)\right) .
$$

The minimal coupling $\exp \left(i \pi \int \Lambda \cup B\right)$ implies that the generator of $\mathbb{Z}_{\mathrm{N},[1]}^{e}$ is precisely $\exp \left(i \pi \int \Lambda\right)$. This explains Eq. (2.9). Notice that integrating out the Lagrangian multiplier $\Lambda$ removes the $\mathrm{U}(1)$ degree of freedom; hence the gauge group is $\mathrm{SO}(3)=\mathrm{PSU}(2)$ [rather than $\mathrm{SU}(2)$ ],

$\frac{\mathrm{U}(2)}{\mathrm{U}(1)}=\frac{\frac{\mathrm{SU}(2) \times \mathrm{U}(1)}{\mathbb{Z}_{2}}}{\mathrm{U}(1)}=\frac{\mathrm{SU}(2)}{\mathbb{Z}_{2}}=\mathrm{PSU}(2)=\mathrm{SO}(3)$,

with the gauge bundle constraint $c_{1}=w_{2}\left(V_{\mathrm{SO}(3)}\right)=B$. Here the second Stiefel-Whitney class $w_{2}\left(V_{\mathrm{SO}(3)}\right) \in$ $H^{2}\left(M, \mathbb{Z}_{2}\right)$ is the obstruction of promoting the $\mathrm{SO}(3)$ bundle to the $\mathrm{SU}(2)$ bundle, which we explain in detail below. The nontrivial SU(2) gauge bundle on a manifold $M$ can be constructed by finding an open cover of $M$ and then gluing together trivial bundles from adjacent open patches via the $S U(2)$ transition functions. Suppose $g_{i j} \in \mathrm{SU}(2)$ is the transition function (which plays the role of gauge transformation) defined on the intersections of two open covers indexed by $i$ and $j$. There is a consistency condition

$$
g_{i j} g_{j k} g_{k i}=1 \in \mathrm{SU}(2)
$$

on the triple overlapping intersections of three open patches indexed by $i, j$, and $k$. However, the consistency condition of the $\mathrm{SO}(3)$ bundle is weaker. Let $h_{i j}$ be the transition function in the $\mathrm{SO}(3)$ bundle, and $\hat{h}_{i j}$ is the lift of $h_{i j}$ in the SU(2) bundle. Then

$$
h_{i j} h_{j k} h_{k i}=1 \in \mathrm{SO}(3) \text {, }
$$

while

$$
\hat{h}_{i j} \hat{h}_{j k} \hat{h}_{k l}=\exp \left(\mathrm{i} \pi w_{i j k}\left(V_{\mathrm{SO}(3)}\right)\right) \in\{ \pm 1\} \subset \mathrm{SU}(2) .
$$

The $w_{i j k}\left(V_{\mathrm{SO}(3)}\right) \in \mathbb{Z}_{2}$ is related to $w_{2}\left(V_{\mathrm{SO}(3)}\right)$ evaluated on the simplex $(i j k) .{ }^{14}$ Thus the $\mathrm{SO}(3)$ bundle

\footnotetext{
${ }^{14}$ The patch $i$ is dual to a zero-simplex $i$ in the dual cell decomposition of spacetime. The intersection of two patches $i$ and $j$ is dual to a one-simplex $(i j)$ in the dual cell decomposition of $M$. The intersection of the patches $i, j$ and $k$ is dual to a twosimplex $(i j k)$ in the dual cell decomposition of $M$.
}

can be lifted to an $\mathrm{SU}(2)$ bundle only when $w_{2}\left(V_{\mathrm{SO}(3)}\right)$ is trivial, i.e., the $\mathbb{Z}_{\mathrm{N},[1]}^{e}$ background field $B \in$ $H^{2}\left(M, \mathbb{Z}_{2}\right)$ is trivial. Namely, activating $B$ allows us to study the $\mathrm{SU}(2)$ gauge theory with the nontrivial $\mathrm{SO}(3)$-gauge bundle. In short,

$$
\begin{aligned}
\frac{\mathrm{d} \hat{A}}{2 \pi} & =c_{1}\left(V_{\mathrm{U}(2)}\right)=B=w_{2}\left(V_{\mathrm{PSU}(2)}\right)=w_{2}\left(V_{\mathrm{SO}(3)}\right) \\
& =w_{2}(E) \bmod 2,
\end{aligned}
$$

and we learn that the SU(2) gauge theory coupled to a background $B$ field can be regarded as a path integral summing over the $\mathrm{SO}(3)$ gauge bundle $E$ subject to the gauge bundle constraint $B=$ $w_{2}\left(V_{\mathrm{PSU}(2)}\right)=w_{2}\left(V_{\mathrm{SO}(3)}\right)=w_{2}(E) \bmod 2$. We will soon propose a new generalization of the gauge bundle constraint of Eq. (2.15) on unorientable or nonspin manifolds. See Eq. (2.25) in Sec. II B.

Coupling to $\mathbb{Z}_{2,[1]}^{e}$ background field $B$ allows one to say more on various line and surface operators. First, one can use $B$ to construct a magnetic two-surface $U_{m}=\exp \left(\mathrm{i} \pi \int_{\Sigma} w_{2}\left(V_{\mathrm{PSU}(2)}\right)\right)=\exp \left(\mathrm{i} \pi \int_{\Sigma} B\right)$. When $\Sigma$ is a surface with a boundary, a Wilson line $W_{e}^{\text {fund }}$ in the fundamental representation (below, we will simply use $W_{e}$ for simplicity) can be supported on the boundary so that $W_{e} U_{m}$ is invariant under the background gauge transformation $B \rightarrow B+\delta \lambda$. Second, when the surface $\Sigma$ of the electric two-surface operator $U_{e}$, Eq. (2.9), has a boundary $\partial \Sigma$, a 't Hooft line $T_{m}$ can be supported on $\partial \Sigma$. Since $U_{e}$ is dynamical in the SU(2) gauge theory, the 't Hooft line $T_{m}$ is not a genuine line operator, and $T_{m}$ has to live on the boundary of $U_{e}$. Thus the 't Hooft line $T_{m}$ as the world line of the probe background magnetic monopole must be attached with the dynamical and detectable open Dirac string, which is visible by $W_{e}$. The closed two world sheet of detectable Dirac string forms the $U_{e}$ operator. This can be seen from the correlation function

$$
\begin{aligned}
\left\langle W_{e} U_{e}\right\rangle & =\left\langle\operatorname{Tr}_{\mathrm{R}}\left(\operatorname{Pexp}\left(\mathrm{i} \oint_{\gamma^{1}} a\right)\right) \exp \left(\mathrm{i} \pi \oint_{\Sigma^{2}} \Lambda\right)\right\rangle \\
& =\exp \left(\frac{\mathrm{i} 2 \pi}{\mathrm{N}} \operatorname{Lk}\left(\gamma^{1}, \Sigma^{2}\right)\right),
\end{aligned}
$$

where $\mathrm{R}$ stands for the fundamental representation. $\operatorname{Lk}\left(\gamma^{1}, \Sigma^{2}\right)$ is the linking number between $\gamma^{1}$ and $\Sigma^{2}$.

From the Hamiltonian point of view, the spatial Wilson line operator $\hat{W}_{e}$ and the spatial 't Hooft operator $\hat{T}_{m}$ (as two canonically quantized line operators) in the $\mathrm{SU}(\mathrm{N})$ gauge theory satisfy the commutation relation [70] 


$$
\begin{aligned}
\hat{W}_{e}\left(\gamma^{1}\right) \hat{T}_{m}\left(\gamma^{1^{\prime}}\right)= & \exp \left(\frac{\mathrm{i} 2 \pi}{\mathrm{N}} \operatorname{Lk}\left(\gamma^{1}, \gamma^{1 \prime}\right)\right) \\
& \times \hat{T}_{m}\left(\gamma^{1^{\prime}}\right) \hat{W}_{e}\left(\gamma^{1}\right),
\end{aligned}
$$

where $\operatorname{Lk}\left(\gamma^{1}, \gamma^{1 \prime}\right)$ is the linking number between $\gamma^{1}$ and $\gamma^{1 \prime}$ in the $3 \mathrm{~d}$ space. For the SU(2) YM, Eq. (2.17) reduces to

$$
\hat{W}_{e}\left(\gamma^{1}\right) \hat{T}_{m}\left(\gamma^{1^{\prime}}\right)=(-1)^{\mathrm{Lk}\left(\gamma^{1}, \gamma^{1 \prime}\right)} \hat{T}_{m}\left(\gamma^{1^{\prime}}\right) \hat{W}_{e}\left(\gamma^{1}\right) .
$$

The noncommutative nature of Eq. (2.17) implies that $W_{e}$ and $T_{m}$ are not mutually local, which is consistent with the fact that $W_{e}$ is a genuine line operator while $T_{m}$ is not a genuine line operator as discussed in the last paragraph.

(5) The full symmetry $\mathbb{Z}_{2}^{T} \times \mathbb{Z}_{2,[1]}^{e}$ : The full symmetry of the SU(2) YM theory relevant in our study is $\mathbb{Z}_{2}^{T} \times \mathbb{Z}_{2,[1]}^{e}{ }^{15}$ The $\mathbb{Z}_{2}^{T}$ symmetry implies the spacetime symmetry has an orthogonal group $\mathrm{O}(d)$ via a short exact sequence extension $1 \rightarrow \mathrm{SO}(d) \rightarrow$ $\mathrm{O}(d) \rightarrow \mathbb{Z}_{2}^{T} \rightarrow 1$ where $\mathrm{SO}(d)$ is the spacetime rotation symmetry. Knowing the full relevant global symmetry, $\mathbb{Z}_{2}^{T} \times \mathbb{Z}_{2,[1]}^{e}$, we can classify the 't Hooft anomalies based on Thom-Madsen-Tillmann-FreedHopkins bordism spectra and cobordism theory [41-43]. In terms of a bordism group $\Omega_{d}^{\mathbb{G}}$ (more precisely, we focus on the torsion part $\Omega_{d, \text { tor }}^{\mathbb{G}}$ ), the classification of $4 d$ 't Hooft anomalies for $4 d$ SU(2) YM can be written as linear combinations of bordism invariants $\Omega_{d}^{\mathbb{G}}=\Omega_{d}^{\mathrm{O}}\left(\mathrm{B}^{2} \mathbb{Z}_{2}\right)$ for $d=5[8,10]$. [We leave the details of bordism invariants for later in Eq. (2.40) and in Sec. II C.]

\section{B. Derivation of new higher anomalies of SU(2) Yang-Mills theory at $\theta=\pi$ on unorientable manifolds}

We start with the SU(2) Yang-Mills theory with $\theta=\pi$, denoted $\mathrm{SU}(2)_{\theta=\pi}$. The Euclidean action $\mathbf{S}_{E}$ is

$$
\mathbf{S}_{E}\left[M^{4}\right]=\frac{1}{g^{2}} \int_{M^{4}} \operatorname{Tr}(F \wedge \star F)-\frac{\mathrm{i} \theta}{8 \pi^{2}} \int_{M^{4}} \operatorname{Tr}(F \wedge F) .
$$

Since the anomaly is a renormalization group flow invariant, in the following discussion, the kinetic term that is proportional to the running coupling constant $1 / g^{2}$ will not play a role. Hence we only consider the second term in Eq. (2.18), which we dub the theta term. To probe the anomaly, we turn on the background gauge field $\mathcal{B}$ for the $\mathbb{Z}_{2,[1]}^{e}$ one-form symmetry. Here $\mathcal{B}$ is a $\mathbb{Z}_{2}$-valued two-form

\footnotetext{
${ }^{15}$ Since $\mathbb{Z}_{2}^{C T} \times \mathbb{Z}_{2,[1]}^{e}$ and $\mathbb{Z}_{2}^{T} \times \mathbb{Z}_{2,[1]}^{e}$ differ by a SU(2) gauge transformation, we only discuss $\mathbb{Z}_{2}^{T} \times \mathbb{Z}_{2,[1]}^{e}$.
}

gauge field with $\oint_{\Sigma} \mathcal{B}=\pi \mathbb{Z}$ for any closed surface $\Sigma$. The two-form gauge field $\mathcal{B}$ is related to the two-cochain $B$ via $\mathcal{B} \sim \pi B$, and we also convert the wedge product $\wedge$ to the cup product $U$ when the action is written in terms of cochains. To couple the SU(2) YM theory to the background gauge field $\mathcal{B}$, we promote the $\mathrm{SU}(2)$ gauge field $a$ to a $\mathrm{U}(2)$ gauge field $\hat{a}$, and the theta term at $\theta=\pi$ reads $^{16}$

$$
\frac{\theta}{8 \pi^{2}} \int_{M^{4}} \operatorname{Tr}\left(\left(\hat{F}-\mathcal{B} \mathbb{I}_{2}\right) \wedge\left(\hat{F}-\mathcal{B} \mathbb{I}_{2}\right)\right)
$$

where $\hat{F}=\mathrm{d} \hat{a}-\mathrm{i} \hat{a} \wedge \hat{a}$ is the $\mathrm{U}(2)$ field strength, and $\mathbb{I}_{2}$ is the two-dimensional identity matrix. To restore the SU(2) gauge field, the $U(2)$ field strength should satisfy the gauge bundle constraint

$$
\begin{aligned}
\frac{\operatorname{Tr} \hat{F}}{2 \pi} & =\frac{2 \mathcal{B}}{2 \pi}=B=w_{2}\left(V_{\mathrm{PSU}(2)}\right)=w_{2}\left(V_{\mathrm{SO}(3)}\right) \\
& =w_{2}(E) \bmod 2 .
\end{aligned}
$$

Here $w_{2}\left(V_{\mathrm{PSU}(2)}\right)=w_{2}\left(V_{\mathrm{SO}(3)}\right)$ is the Stiefel-Whitney class of the associated vector bundle of the $\mathrm{PSU}(2)=\mathrm{SO}(3)$ [the principal gauge bundle $E$ of $\mathrm{PSU}(2)=\mathrm{SO}(3)]$.

To activate the background field for the time-reversal symmetry, we formulate Eq. (2.19) on an unorientable manifold $M^{4}$. On an unorientable manifold, the top differential form is not well defined, due to the lack of the volume form whose definition needs an orientation. To make sense of Eq. (2.19) on an unorientable manifold, we reformulate it in terms of the Chern characteristic classes. We denote the $j$ th Chern class of the $\mathrm{U}(\mathrm{N})$ gauge bundle as $c_{j}\left(V_{\mathrm{U}(\mathrm{N})}\right)$. For $j=1$, 2, we have

$$
\begin{aligned}
& c_{1}\left(V_{\mathrm{U}(\mathrm{N})}\right)=\frac{\operatorname{Tr} \hat{F}}{2 \pi}, \\
& c_{2}\left(V_{\mathrm{U}(\mathrm{N})}\right)=-\frac{1}{8 \pi^{2}} \operatorname{Tr}(\hat{F} \wedge \hat{F})+\frac{1}{8 \pi^{2}}(\operatorname{Tr} \hat{F}) \wedge(\operatorname{Tr} \hat{F}) .
\end{aligned}
$$

Replacing $\frac{1}{8 \pi^{2}} \operatorname{Tr}(\hat{F} \wedge \hat{F})$ by $\quad \frac{c_{1} \cup c_{1}}{2}-c_{2}$, we rewrite Eq. (2.19) as ${ }^{17}$

\footnotetext{
${ }^{16}$ The topological term for the Euclidean action $S_{\mathbf{E} \text {,topological }}$ in the Euclidean partition function $\mathbf{Z}=\exp \left(-S_{\mathbf{E} \text {,topological }}\right)$ contains a factor of imaginary i, namely $\mathbf{S}_{E}=-\mathrm{i}(\cdots)$ in Eq. (2.18). However, by converting $\exp \left(-\mathbf{S}_{E}\right)=\exp (\mathrm{iS})$, we have the following Minkowski $\mathbf{S}$ in Eq. (2.19).

${ }^{17}$ Some of the mathematical-oriented readers may wonder how to rigorously define Eq. (2.19)'s $\frac{\pi}{8 \pi^{2}} \int_{M^{4}} \operatorname{Tr}\left(\left(\hat{F}-\mathcal{B} \mathbb{I}_{2}\right) \wedge(\hat{F}-\right.$ $\left.\left.\mathcal{B} \mathbb{I}_{2}\right)\right)$ to a term $\frac{\pi}{8 \pi^{2}} \int_{M^{4}} \operatorname{Tr}\left(\left(\hat{F}-\pi B \mathbb{I}_{2}\right) \wedge\left(\hat{F}-\pi B \mathbb{I}_{2}\right)\right)$ with the continuous differential form $\hat{F}$ coupling to a discrete cohomology class $B \in H^{2}\left(M, \mathbb{Z}_{2}\right)$. In fact, the physics way to interpret this coupling is related to the coupling between QFT to TQFT explained in [69]. More formally, we can also implement mathematical methods [71] to formulate such couplings. J. W. thanks Shing-Tung Yau for insightful conversations on this method [71]
} 


$$
\frac{\pi}{8 \pi^{2}} \int_{M^{4}}\left(\operatorname{Tr}(\hat{F} \wedge \hat{F})-2 \operatorname{Tr}(\hat{F}) \wedge \mathcal{B}+\operatorname{Tr}\left(\mathbb{I}_{2}\right) \mathcal{B} \wedge \mathcal{B}\right)=\pi \int_{M^{4}}\left(\frac{1}{8 \pi^{2}} \operatorname{Tr}(\hat{F} \wedge \hat{F})-\frac{1}{2} \operatorname{Tr}\left(\frac{\hat{F}}{2 \pi}\right) \wedge \frac{\mathcal{B}}{\pi}+\frac{1}{4} \frac{\mathcal{B}}{\pi} \wedge \frac{\mathcal{B}}{\pi}\right)
$$

Using Eq. (2.21), Eq. (2.22) can be reinterpreted as

$$
\pi \int_{M^{4}}\left(-c_{2}\left(V_{\mathrm{U}(2)}\right)+\frac{c_{1}\left(V_{\mathrm{U}(2)}\right) \cup c_{1}\left(V_{\mathrm{U}(2)}\right)}{2}-\frac{1}{2} c_{1}\left(V_{\mathrm{U}(2)}\right) \cup B+\frac{\mathcal{P}(B)}{4}\right),
$$

where $\mathcal{P}(B)$ is the Pontryagin square ${ }^{18}$ of $B$.

Note that Eq. (2.23) is not well defined even on an orientable manifold. In Sec. II D, we resolve this problem for the torsion-free oriented manifolds $M$. Yet, Eq. (2.23) is also not well defined on an unorientable manifold. In general, if $M$ is a $d$-dimensional unorientable manifold and $\omega$ is a $d$ cocycle, $\pi \int_{M} \omega \bmod 2 \pi$ is well defined only when $\omega$ is valued in $\mathbb{Z}_{2}{ }^{19}$ Since $c_{2}\left(V_{\mathrm{U}(2)}\right) \in H^{4}\left(M^{4}, \mathbb{Z}\right)$ is integer valued, the first term in Eq. (2.23) makes sense when $M^{4}$ is unorientable. However, the other terms are fractional; hence the integral of those terms does not make sense if $M^{4}$ is unorientable. To make sense of Eq. (2.23), we actually need to define it on both the unorientable $M^{4}$ and an unorientable $M^{5}$ such that $\partial M^{5}=M^{4} \cdot{ }^{20}$ To proceed, we extend the integer valued cohomology class $c_{1}\left(V_{\mathrm{U}(2)}\right)$ on $M^{4}$ to an integer valued cochain $\tilde{c}_{1}\left(V_{\mathrm{U}(2)}\right)$ on $M^{5}$. Note that $\tilde{c}_{1}\left(V_{\mathrm{U}(2)}\right)$ on $M^{5}$ does not have to be an element in $H^{2}\left(M^{5}, \mathbb{Z}\right)$, i.e., $\delta \tilde{c}_{1}\left(V_{\mathrm{U}(2)}\right)=0$ does not have to hold onto $M^{5}$. The requirement of $\tilde{c}_{1}\left(V_{\mathrm{U}(2)}\right)$ will be imposed later by the gauge bundle constraint. The extension means, in particular, that when restricting $\tilde{c}_{1}\left(V_{\mathrm{U}(2)}\right)$ to $M^{4}$, it reduces to a $\mathbb{Z}$-valued cohomology class $c_{1}\left(V_{\mathrm{U}(2)}\right)$. We further extend the $\mathbb{Z}_{2}$-valued cohomology class $B$ on $M^{4}$ to a $\mathbb{Z}_{2}$-valued cohomology class on $M^{5}$, and for simplicity, we use the same notation $B$ on $M^{5}$ as well. Thus we define Eq. (2.23) as follows:

$$
\begin{aligned}
& -\pi \int_{M^{4}} c_{2}\left(V_{\mathrm{U}(2)}\right)+\pi \int_{M^{5}} \delta\left(\frac{c_{1}\left(V_{\mathrm{U}(2)}\right) \cup c_{1}\left(V_{\mathrm{U}(2)}\right)}{2}-\frac{1}{2} c_{1}\left(V_{\mathrm{U}(2)}\right) \cup B+\frac{\mathcal{P}(B)}{4}\right) \\
& =-\pi \int_{M^{4}} c_{2}\left(V_{\mathrm{U}(2)}\right)+\pi \int_{M^{5}}\left(\frac{\delta\left(\tilde{c}_{1}\left(V_{\mathrm{U}(2)}\right) \cup \tilde{c}_{1}\left(V_{\mathrm{U}(2)}\right)\right)}{2}-\frac{1}{2} \delta\left(\tilde{c}_{1}\left(V_{\mathrm{U}(2)}\right) \cup B\right)+\frac{\delta \mathcal{P}(B)}{4}\right),
\end{aligned}
$$

with the background field $B$ properly extended to $M^{5}$. Here $\delta$ is a coboundary operator, such that we apply $\int_{M^{4}}(\cdots)=\int_{M^{5}} \delta(\cdots)$ from (2.23) to (2.24). To make sure that the integral on an unorientable $M^{5}$ is well defined, and also independent of the dynamical gauge field, we need to utilize the gauge bundle constraint, which relates $\tilde{c}_{1}\left(V_{\mathrm{U}(2)}\right)$ with the background gauge fields $B, w_{1}(T M)$ and $w_{2}(T M)$. Below, we will see that the fivedimensional integral does not depend on the dynamical

\footnotetext{
${ }^{18}$ Notice it is crucial to treat $\frac{\pi}{8 \pi^{2}} \int_{M^{4}} \operatorname{Tr}\left(\mathcal{B I}_{2} \wedge \mathcal{B I}_{2}\right)=\frac{\pi}{8} \int_{M^{4}} B \wedge$ $B\left(\operatorname{Tr}\left(\mathbb{I}_{2}\right)\right)=\frac{2 \pi}{8} \int_{M^{4}} B \wedge B=\frac{\pi}{4} \int_{M^{4}} B \wedge B \simeq \frac{\pi}{4} \int_{M^{4}} \mathcal{P}(B)$ as the more precise rewriting for the later purposes. The $\mathcal{P}(B)$ denotes the Pontryagin square; e.g., see Ref. [10].

${ }^{19}$ Using the definition of the fundamental class of an unorientable manifold $M$, i.e., $[M]$, one has $\int_{M} \omega=\langle\omega,[M]\rangle$ where $\langle\omega,[M]\rangle$ is the $\mathbb{Z}_{2}$ valued pairing between $\omega$ and $[M] \in H_{d}\left(M, \mathbb{Z}_{2}\right)$.

${ }^{20}$ Note that if $M^{5}$ is orientable, then $M^{4}$ must be orientable. Conversely, if $M^{4}$ is unorientable, $M^{5}$ must be unorientable. However, if $M^{4}$ is orientable, $M^{5}$ can be orientable or unorientable.
}

gauge fields due to the gauge bundle constraints. Hence the $5 \mathrm{~d}$ integral is an invertible TQFT whose partition function is a local function of the background fields. In summary, we find that in order to make sense of the theta term of the $S U(2) Y M$ theory with the background fields on an unorientable manifold, one needs to treat the $\mathrm{SU}(2)_{\theta=\pi}$ YM theory as a $4 \mathrm{~d}-5 \mathrm{~d}$ coupled system. This is a manifestation of the mixed 't Hooft anomaly between the one-form global symmetry $\mathbb{Z}_{2,[1]}^{e}$ and the time-reversal symmetry $\mathbb{Z}_{2}^{T}$.

On an unorientable manifold $M=M^{4}$, the $w_{1}(T M)$ is nontrivial and one can treat it as the background gauge field for the time-reversal symmetry. This allows us to modify the gauge bundle constraint Eq. (2.20) by an additional term $K_{1} w_{1}(T M)^{2}$, with $K_{1}=0,1 \in \mathbb{Z}_{2}$. Furthermore, we are also allowed to consider the manifold $M$ with nontrivial $w_{2}(T M)$ since the underlying manifold does not necessarily allow a spin/pin structure; hence we activate the term $K_{2} w_{2}(T M)$ with $K_{2}=0,1 \in \mathbb{Z}_{2}$. In summary, there are four choices of gauge bundle constraints labeled by the pair $\left(K_{1}, K_{2}\right) \in\left(\mathbb{Z}_{2}, \mathbb{Z}_{2}\right)$ as 


$$
c_{1}\left(V_{\mathrm{U}(2)}\right)=B+K_{1} w_{1}(T M)^{2}+K_{2} w_{2}(T M)=w_{2}\left(V_{\mathrm{PSU}(2)}\right)=w_{2}\left(V_{\mathrm{SO}(3)}\right)=w_{2}(E) \bmod 2, \quad K_{1,2} \in \mathbb{Z}_{2} .
$$

This is a nontrivial constraint between the gauge bundle $E$, the spacetime tangent bundle $T M$, and the background field $B$. The value of $K_{1,2}$ has physical consequences: when $K_{1}=0,1$, the SU(2) gauge charge [in the fundamental representation of $\mathrm{SU}(2)]$ is a Kramers singlet $\left(T^{2}=+1\right)$ or a Kramers doublet $\left(T^{2}=-1\right)$ under time-reversal transformation ${ }^{21}$; when $K_{2}=0,1$, the $\mathrm{SU}(2)$ gauge charge is a boson (spin statistics as an integer spin) or a fermion (spin statistics as a half-integer spin). More details about the Wilson line properties are derived in Sec. III.

The gauge bundle constraint Eq. (2.25) is defined on $M^{4}$. We would like to promote it to $M^{5}$ as follows:

$$
\tilde{c}_{1}\left(V_{\mathrm{U}(2)}\right)=B+K_{1} w_{1}(T M)^{2}+K_{2} w_{2}(T M) \bmod 2, \quad K_{1,2} \in \mathbb{Z}_{2} .
$$

Equation (2.26) imposes additional constraints on $\tilde{c}_{1}\left(V_{\mathrm{U}(2)}\right)$. Since $B, w_{1}(T M)^{2}$ and $w_{2}(T M)$ are $\mathbb{Z}_{2}$ cohomology on $M^{5}$, $\tilde{c}_{1}\left(V_{\mathrm{U}(2)}\right)$ is equivalent to a $\mathbb{Z}_{2}$-valued cohomology $H^{2}\left(M^{5}, \mathbb{Z}_{2}\right) \bmod 2$ (although it is not a $\mathbb{Z}$-valued cohomology), i.e., $\delta \tilde{c}_{1}\left(V_{\mathrm{U}(2)}\right)=0 \bmod 2$.

We further apply the gauge bundle constraint Eq. (2.25) to the five-dimensional integral Eq. (2.24). We should be aware that the five manifold $M^{5}$ has a boundary $M^{4}$. Here we summarize some helpful formulas and mathematical definitions in a footnote. ${ }^{22}$ Since $\tilde{c}_{1}\left(V_{\mathrm{U}(2)}\right)$ mod 2 is in $H^{2}\left(M, \mathbb{Z}_{2}\right)$, it makes sense to define its Steenrod square $\operatorname{Sq}^{1} \tilde{c}_{1}\left(V_{\mathrm{U}(2)}\right)$. Then the $5 \mathrm{~d}$ integral in Eq. (2.24) can be written as

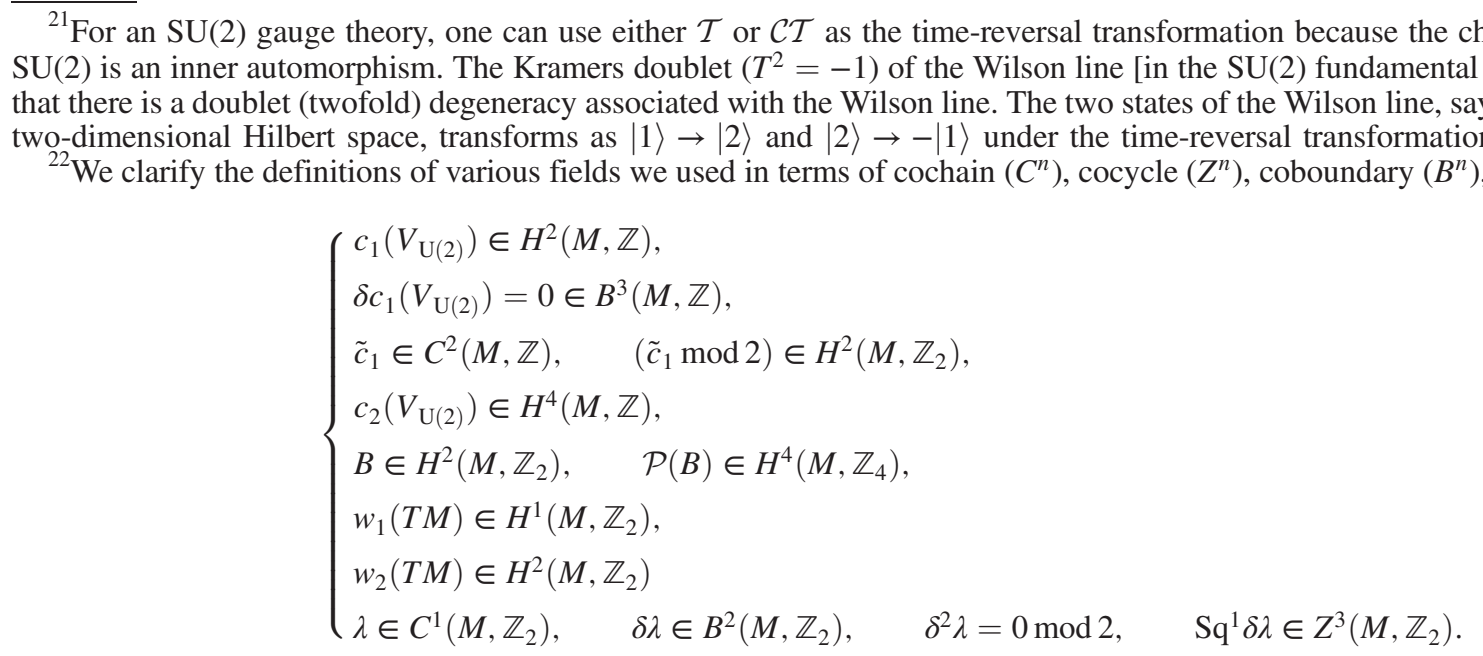

Here $C^{n}$ stands for the $n$th cochain, $H^{n}$ for the $n$th cohomology, $Z^{n}$ for the $n$th cocycle, and $B^{n}$ for the $n$th coboundary. When discussing the cup products, there are subtle distinctions between (1) cohomology classes in $H^{n}$, (2) cocycles in $Z^{n}$, and (3) cochains in $C^{n}$, which we enumerate below:

(1) The cup product between two cohomology classes $u \in H^{p}\left(M, \mathbb{Z}_{2}\right), v \in H^{q}\left(M, \mathbb{Z}_{2}\right)$ are supercommutative, i.e.,

$$
u \cup v=(-1)^{p q} v \cup u .
$$

(2) The cup products between two cocycles are not supercommutative. If $u \in Z^{p}$ and $v \in Z^{q}$ are general $p$ th and $q$ th cocycles, their commutation relation is governed by Steenrod's relation [61]

$$
u \cup v-(-1)^{p q} v \cup u=(-1)^{p+q-1}\left(\delta(u \cup v v)-\delta u \cup \underset{1}{ } v-(-1)^{p} u \cup \delta v\right)=(-1)^{p+q-1} \delta(u \cup v),
$$

where we have used the cocycle condition $\delta u=0 \bmod 2, \delta v=0 \bmod 2$.

(3) The cup products between two cochains satisfy Steenrod's relation [61]

$$
\begin{gathered}
\delta(u \cup v)=(-1)^{p+q-i} u \cup_{i-1}^{\cup} v+(-1)^{p q+p+q}{\underset{i-1}{\cup}}_{i} u+\delta u \bigcup_{i} v+(-1)^{p} u \cup_{i} \delta v, \\
\delta(u \cup v)=\delta u \cup v+(-1)^{p} u \cup \delta v,
\end{gathered}
$$

where $u \in C^{p}$ and $v \in C^{q}$ are general $p$ th and $q$ th cochains.

In this section, all the calculations still go through if we regard the $B$ field as a $\mathbb{Z}_{2}$ two-cocycle, because we did not use the supercommutativity. 


$$
\begin{aligned}
\mathbf{S}_{\mathrm{anom}} \equiv & \pi \int_{M^{5}} \delta\left(\frac{\mathcal{P}(B)}{4}-\frac{1}{2} \tilde{c}_{1}\left(V_{\mathrm{U}(2)}\right) \cup B+\frac{\tilde{c}_{1}\left(V_{\mathrm{U}(2)}\right) \cup \tilde{c}_{1}\left(V_{\mathrm{U}(2)}\right)}{2}\right) \\
= & \pi \int_{M^{5}} \frac{\delta \mathcal{P}(B)}{4}-\frac{1}{2} \tilde{c}_{1}\left(V_{\mathrm{U}(2)}\right) \cup \delta B-\frac{1}{2} \delta \tilde{c}_{1}\left(V_{\mathrm{U}(2)}\right) \cup B+\frac{\delta \tilde{c}_{1}\left(V_{\mathrm{U}(2)}\right) \cup \tilde{c}_{1}\left(V_{\mathrm{U}(2)}\right)}{2}+\frac{\tilde{c}_{1}\left(V_{\mathrm{U}(2)}\right) \cup \delta \tilde{c}_{1}\left(V_{\mathrm{U}(2)}\right)}{2} \\
= & \pi \int_{M^{5}} B \mathrm{Sq}^{1} B+\mathrm{Sq}^{2} \mathrm{Sq}^{1} B-\tilde{c}_{1}\left(V_{\mathrm{U}(2)}\right) \cup \mathrm{Sq}^{1} B-\mathrm{Sq}^{1} \tilde{c}_{1}\left(V_{\mathrm{U}(2)}\right) \cup B+\mathrm{Sq}^{1} \tilde{c}_{1}\left(V_{\mathrm{U}(2)}\right) \cup \tilde{c}_{1}\left(V_{\mathrm{U}(2)}\right) \\
& +\tilde{c}_{1}\left(V_{\mathrm{U}(2)}\right) \cup \mathrm{Sq}^{1} \tilde{c}_{1}\left(V_{\mathrm{U}(2)}\right) \\
= & \pi \int_{M^{5}} B \mathrm{Sq}^{1} B+\mathrm{Sq}^{2} \mathrm{Sq}^{1} B-\left(B+K_{1} w_{1}(T M)^{2}+K_{2} w_{2}(T M)\right) \cup \mathrm{Sq}^{1} B-\left(\mathrm{Sq}^{1} B+K_{2} \mathrm{Sq}^{1} w_{2}(T M)\right) \cup B \\
& +\left(\mathrm{Sq}^{1} B+K_{2} \mathrm{Sq}^{1} w_{2}(T M)\right) \cup\left(B+K_{1} w_{1}(T M)^{2}+K_{2} w_{2}(T M)\right) \\
& +\left(B+K_{1} w_{1}(T M)^{2}+K_{2} w_{2}(T M)\right) \cup\left(\mathrm{Sq}^{1} B+K_{2} \mathrm{Sq}^{1} w_{2}(T M)\right) \\
= & \pi \int_{M^{5}} B \mathrm{Sq}^{1} B+\mathrm{Sq}^{2} \mathrm{Sq}^{1} B+K_{1} \mathrm{Sq}^{1} B \cup w_{1}(T M)^{2}+K_{2} \mathrm{Sq}^{1}\left(B \cup w_{2}(T M)\right) \\
& +K_{2}\left(\left(K_{1} w_{1}^{2}+K_{2} w_{2}\right) \cup \mathrm{Sq}^{1} w_{2}+\mathrm{Sq}^{1} w_{2} \cup\left(K_{1} w_{1}^{2}+K_{2} w_{2}\right)\right) \\
= & \pi \int_{M^{5}} B \mathrm{Sq}^{1} B+\mathrm{Sq}^{2} \mathrm{Sq}^{1} B+K_{1} \mathrm{Sq}^{1} B \cup w_{1}(T M)^{2}+K_{2} \mathrm{Sq}^{1}\left(B \cup w_{2}(T M)\right) .
\end{aligned}
$$

In the first equality, we simply stated the initial definition. In the second equality, we plugged in the coboundary operator $\delta$. In the third equality, we used Eq. (2.31) and replaced $\delta / 2$ by $\mathrm{Sq}^{1}$ which is valid for $\mathbb{Z}_{2}$-valued cocycles. We also used the identity $\frac{\delta \mathcal{P}(B)}{4}=B \mathrm{Sq}^{1} B+\mathrm{Sq}^{2} \mathrm{Sq}^{1} B$ since $B$ is a $\mathbb{Z}_{2}$-valued two-cocycle [8]. ${ }^{23}$ In the fourth equality, we plug in the gauge bundle constraint Eq. (2.25). Equation (2.25) also implies $\mathrm{Sq}^{1} c_{1}\left(V_{\mathrm{U}(2)}\right)=\mathrm{Sq}^{1} B+$ $K_{2} \mathrm{Sq}^{1} w_{2}(T M)$. In the fifth equality, we used $\mathrm{Sq}^{1}(B \cup$ $\left.w_{1}(T M)^{2}\right)=\left(\mathrm{Sq}^{1} B\right) \cup w_{1}(T M)^{2}+B \cup \mathrm{Sq}^{1}\left(w_{1}(T M)^{2}\right)$. In the last equality, we used $\left(\left(K_{1} w_{1}^{2}+K_{2} w_{2}\right) \cup \mathrm{Sq}^{1} w_{2}+\right.$ $\left.\mathrm{Sq}^{1} w_{2} \cup\left(K_{1} w_{1}^{2}+K_{2} w_{2}\right)\right)=0 \bmod 2$ since the StiefelWhitney classes are supercommutative.

Several comments are in order:

(1) As mentioned below Eq. (2.24), $\mathbf{S}_{\text {anom }}$ is a properly quantized integral of the background field $B$ and the Stiefel-Whitney class $w_{i}(T M)$, which is independent of the dynamical U(2) gauge field. Hence $\mathbf{S}_{\text {anom }}$ is an invertible TQFT.

(2) In Eq. (2.24) and Eq. (2.32), the 5d unorientable manifold $M^{5}$ has a boundary $M^{4}$ :

\footnotetext{
${ }^{23}$ For a two-cocycle $B$, the following equality holds:

$$
\begin{aligned}
& \frac{1}{4} \delta \mathcal{P}(B)=\frac{1}{4} \delta(B \cup B+\underset{1}{B \cup \delta B}) \\
& =\left(\frac{1}{2} \delta B\right) \cup B+\left(\frac{1}{2} \delta B\right)_{1} \cup\left(\frac{1}{2} \delta B\right) \\
& =B \mathrm{Sq}^{1} B+\mathrm{Sq}^{1} B \cup \mathrm{Sq}^{1} B=B \mathrm{Sq}^{1} B+\mathrm{Sq}^{2} \mathrm{Sq}^{1} B \text {. }
\end{aligned}
$$

See Eq. (124) in [8] for further details. (a) If $M^{5}$ does not have a boundary, the term $K_{2} \operatorname{Sq}^{1}\left(B \cup w_{2}(T M)\right)$ vanishes, due to

$$
\begin{aligned}
K_{2}\left(w_{2}(T M)\right. & \left.\cup \mathrm{Sq}^{1} B+\mathrm{Sq}^{1} w_{2}(T M) \cup B\right) \\
& =K_{2} \operatorname{Sq}^{1}\left(w_{2}(T M) \cup B\right) \\
& =K_{2} w_{1}(T M) w_{2}(T M) \cup B \\
& =K_{2} \mathrm{Sq}^{3} B=K_{2} u_{3} B=0 \bmod 2 \pi
\end{aligned}
$$

In the last step, we have used the $\mathrm{Wu}$ formula $u_{3} \equiv u_{3}(T M)=w_{1}(T M) w_{2}(T M)=0 \bmod 2$, on a closed five manifold. Hence Eq. (2.32) simplifies to

$\pi \int_{M^{5}} B \mathrm{Sq}^{1} B+\mathrm{Sq}^{2} \mathrm{Sq}^{1} B+K_{1} w_{1}(T M)^{2} \cup \mathrm{Sq}^{1} B$.

(b) If $M^{5}$ has a boundary, $K_{2} \pi \mathrm{Sq}^{1}\left(w_{2}(T M) \cup B\right)$ transforms nontrivially under the background gauge transformation $B \rightarrow B+\delta \lambda$,

$$
\begin{aligned}
& K_{2} \pi \int_{M^{5}} \operatorname{Sq}^{1}\left(w_{2}(T M) \cup B\right) \\
& \rightarrow K_{2} \pi \int_{M^{5}} \operatorname{Sq}^{1}\left(w_{2}(T M) \cup B\right) \\
& \quad+K_{2} \pi \int_{M^{5}} \operatorname{Sq}^{1}\left(w_{2}(T M) \cup \delta \lambda\right) .
\end{aligned}
$$


This compensates the noninvariance of the $4 \mathrm{~d}$ theory under $B \rightarrow B+\delta \lambda$. Thus although the $K_{2}$ terms vanish when $M^{5}$ is a closed manifold, when $M^{5}$ has a boundary, it is crucial to keep track of this term.

(c) We can show that the term $K_{2} \int_{M^{5}} \mathrm{Sq}^{1} \times$ $\left(w_{2}(T M) \cup B\right)$ is well defined in $4 \mathrm{~d}$ by showing that this terms depends only on the $4 \mathrm{~d}$ boundary $\partial M^{5}$. The triviality of $\int_{M^{5}} K_{2} \mathrm{Sq}^{1}\left(w_{2}(T M) \cup\right.$ $B)=0$ on a closed $M^{5}$ implies that when the $5 \mathrm{~d}$ manifold has a boundary, such a term does not depend on the choice of extension; i.e., given two $5 \mathrm{~d}$ extensions $M^{5}$ and $\tilde{M}^{5}$, we know $K_{2} \int_{M^{5} \cup \tilde{M}^{5}} \mathrm{Sq}^{1}\left(w_{2}(T M) \cup B\right)=0$ because $M^{5} \cup$ $\tilde{M}^{5}$ is closed, and thus we derive $K_{2} \int_{M^{5}} \mathrm{Sq}^{1} \times$ $\left(w_{2}(T M) \cup B\right)=K_{2} \int_{\tilde{M}^{5}} \operatorname{Sq}^{1}\left(w_{2}(T M) \cup B\right)$. Note that when $M^{5}$ has a boundary, $K_{2} \int_{M^{5}} \mathrm{Sq}^{1} \times$ $\left(w_{2}(T M) \cup B\right)$ can be nonzero. This is analogous to the WZW term. See Sec. IIE for further discussions.

(3) The $4 d-5 d$ integral Eq. (2.24) is invariant under a one-form gauge transformation $B \rightarrow B+\delta \lambda$. We will show this explicitly in Sec. II C.

(4) Although $\mathbf{S}_{\text {anom }}$ depends only on $K_{1}$ when $M^{5}$ is closed, we still label it as the $5 \mathrm{~d}$ anomaly polynomial parametrized by $\left(K_{1}, K_{2}\right)$, due to the subtlety that the 5 d integral still depends on $K_{2}$ when $M^{5}$ has a boundary.

To summarize, the partition function of the combined $4 d-5 d$ coupled system

$\mathbf{Z}_{\mathrm{SU}(2)_{\theta=\pi} \mathrm{YM}}^{4 \mathrm{~d}}\left[M^{4} ; B, w_{j}(T M)\right] \cdot \mathbf{Z}^{5 \mathrm{~d}}\left[M^{5} ; B, w_{j}(T M)\right]$

is gauge invariant under the gauge transformation of the background field $B$, and it also makes sense when $M^{4}$ and $M^{5}$ are unorientable, where

$$
\begin{aligned}
\mathbf{Z}_{\mathrm{SU}(2)_{\theta=\pi} \mathrm{YM}}^{4 \mathrm{~d}}\left[M^{4} ; B, w_{j}(T M)\right]= & \int[\mathcal{D} \hat{a}][\mathcal{D} \Lambda] \exp \left(-\frac{1}{g^{2}} \int_{M^{4}} \operatorname{Tr}\left(\left(\hat{F}-\pi B \mathbb{I}_{2}\right) \wedge \star\left(\hat{F}-\pi B \mathbb{I}_{2}\right)\right)\right) \\
& \cdot \exp \left(-\mathrm{i} \pi \int_{M^{4}} c_{2}\left(V_{\mathrm{U}(2)}\right)\right) \cdot \exp \left(\mathrm{i} \pi \int_{M^{4}} \Lambda \cup\left(c_{1}-B-K_{1} w_{1}(T M)^{2}-K_{2} w_{2}(T M)\right)\right)
\end{aligned}
$$

and

$$
\mathbf{Z}^{5 \mathrm{~d}}\left[M^{5} ; B, w_{j}(T M)\right]=\exp \left(\mathrm{i} \pi \int_{M^{5}} B \mathrm{Sq}^{1} B+\mathrm{Sq}^{2} \mathrm{Sq}^{1} B+K_{1} w_{1}(T M)^{2} \cup \mathrm{Sq}^{1} B+K_{2} \operatorname{Sq}^{1}\left(w_{2}(T M) \cup B\right)\right)
$$

The combined $4 \mathrm{~d}-5 \mathrm{~d}$ system is anomaly-free. Equivalently, to couple the background fields of both time-reversal symmetry and the one-form global symmetry $\mathbb{Z}_{2,[1]}^{e}$, the $\mathrm{SU}(2)_{\theta=\pi} \mathrm{YM}$ theory cannot be placed on an unorientable $M^{4}$ only; instead, one needs to place it on the boundary of an unorientable $M^{5}$ which supports a $5 \mathrm{~d}$ invertible TQFT. This is the manifestation of the mixed 't Hooft anomaly between the one-form global symmetry $\mathbb{Z}_{2,[1]}^{e}$ and the timereversal symmetry $\mathbb{Z}_{2}^{T}$.

\section{Anomaly matching of $5 d-4 d$ inflow and $5 d$ cobordism group data}

In this subsection, we identify the $5 \mathrm{~d}$ topological terms Eq. (2.32) with the mathematically well-defined 5d bordism invariants and further explicitly check the invariance of the $4 \mathrm{~d}-5 \mathrm{~d}$ system Eq. (2.36) under $B \rightarrow B+\delta \lambda$.

\section{Identifying $\mathrm{S}_{\mathrm{anom}}$ with 5d cobordism group data}

We compare $\mathbf{S}_{\text {anom }}$ in Eq. (2.32) with the bordism group data given in $[8,10]$. Since the global symmetries of $4 d$
$\mathrm{SU}(2)_{\theta=\pi}$ YM theory are $\mathbb{Z}_{2}^{T} \times \mathbb{Z}_{2,[1]}^{e}$, we compute the $5 \mathrm{~d}$ bordism group ${ }^{24,25}$

$$
\Omega_{5}^{\mathrm{O}}\left(\mathrm{B}^{2} \mathbb{Z}_{2}\right)=\mathbb{Z}_{2}^{4} .
$$

Hence there are four independent generators of the bordism group $\Omega_{5}^{\mathrm{O}}\left(\mathrm{B}^{2} \mathbb{Z}_{2}\right)$,

\footnotetext{
${ }^{24}$ In addition to $[8,10]$, we notice that the oriented version of the bordism group $\Omega_{5}^{\mathrm{SO}}\left(\mathrm{B}^{2} \mathbb{Z}_{2}\right)$ has been studied recently in [72] for different purposes. Here we study instead the unoriented version of the bordism group $\Omega_{5}^{\mathrm{O}}\left(\mathrm{B}^{2} \mathbb{Z}_{2}\right)$, new to the literature. See details in the Appendix.

${ }^{25}$ For an ordinary (zero-form) global symmetry, we denote $G$ as the zero-form global symmetry group. When gauging a zeroform symmetry, we introduce a one-form flat gauge field with a gauge group $G$, whose classifying space is $B G$. For an Abelian group and for a higher symmetry: We denote $G_{[1]}$ as the one-form global symmetry group. When gauging a one-form symmetry, we introduce a two-form flat gauge field with a higher gauge group, whose classifying space is associated with $B(B G)=B^{2} G$. Similarly, for an Abelian $n$-form global symmetry group $G_{[n]}$, we have the associated classifying space $B^{n+1} G$. See [48-50].
} 


$$
\left\{\begin{array}{l}
B \mathrm{Sq}^{1} B \\
\mathrm{Sq}^{2} \mathrm{Sq}^{1} B=\left(w_{2}(T M)+w_{1}(T M)^{2}\right) \mathrm{Sq}^{1} B=\left(w_{3}(T M)+w_{1}(T M)^{3}\right) B \\
w_{1}(T M)^{2} \mathrm{Sq}^{1} B=w_{1}(T M)^{3} B \\
w_{2}(T M) w_{3}(T M),
\end{array}\right.
$$

where the equalities hold only on closed five manifolds. Clearly, $\mathbf{S}_{\text {anom }}$ in Eq. (2.32) is a bordism invariant except the term proportional to $K_{2}$. Setting $K_{2}=0, \mathbf{S}_{\text {anom }}$ is identified with the sum of the first three bordism invariants in Eq. (2.40),

$$
\exp \left(\mathrm{i} \pi \int_{M^{5}}\left(B \mathrm{Sq}^{1} B+\mathrm{Sq}^{2} \mathrm{Sq}^{1} B+K_{1} w_{1}(T M)^{3} B\right)\right) .
$$

As explained in Sec. II B, the fourth term in $\mathbf{S}_{\text {anom }}$ is trivial when $M^{5}$ does not have a boundary. This is consistent with the fact that there is not any bordism invariant of $\Omega_{5}^{\mathrm{O}}\left(\mathrm{B}^{2} \mathbb{Z}_{2}\right)$ of the form $\mathrm{Sq}^{1}\left(w_{2}(T M) \cup B\right)$.

Notice that the last invariant in Eq. $(2.40),{ }^{26}$ i.e., $w_{2}(T M) w_{3}(T M)$, does not participate in the anomaly of $\mathrm{SU}(2)_{\theta=\pi}$ YM. However, it is responsible for the new SU(2) anomaly [57]: 4d SU(2) gauge theory with an odd number of fermion multiplets in representations of isospin $4 r+3 / 2$ of the gauge group is inconsistent, for a nonnegative integer $r$. The theory is nevertheless consistent on certain manifolds with spin or $\operatorname{spin}^{c}$ structure. The new SU(2) anomaly [57] is in contrast to the old SU(2) anomaly [56]. The familiar SU(2) anomaly [56] states that a 4d SU(2) gauge theory with an odd number of fermion multiplets in the isospin $2 r+1 / 2$ representation is inconsistent.

\section{Anomaly matching of $4 d-5 d$ inflow}

We first highlight the distinctions between the derivation of anomalies in [5] and in our Sec. II B:

(i) Reference [5] places the $\mathrm{SU}(2)_{\theta=\pi} \mathrm{YM}$ on an orientable manifold and turns on the two-form background field $\mathcal{B}$ of the one-form symmetry $\mathbb{Z}_{2,[1]}^{e}$ (or a two-cochain $B$ ). By performing a

\footnotetext{
${ }^{26}$ The $w_{2}(T M) w_{3}(T M)$ is a bordism invariant in $\Omega_{5}^{\mathrm{O}}\left(\mathrm{B}^{2} \mathbb{Z}_{2}\right)$, $\Omega_{5}^{\mathrm{O}}(p t), \Omega_{5}^{\mathrm{SO}}(p t)$, and $\Omega_{5}^{\frac{\mathrm{Spin} \times \mathrm{SU}(2)}{\mathbb{Z}_{2}}}$; see [10]. Namely, this $w_{2}(T M) \times$ $w_{3}(T M)$ is not only a topological term respecting a spacetime $\mathrm{O}(d)$ symmetry and one-form $\mathbb{Z}_{2,[1]}^{e}$ symmetry but also a topological term respecting a spacetime $\mathrm{O}(d)$ or $\mathrm{SO}(d)$ symmetry alone, or respecting an enhanced spacetime-internal locked symmetry $\frac{\operatorname{Spin} \times \operatorname{SU}(2)}{\mathbb{Z}_{2}}$. Thus the $4 \mathrm{~d}$ anomaly from $\Omega_{5}^{\frac{\operatorname{Spin} \times \mathrm{SU}(2)}{\mathbb{Z}_{2}}}$ is a signature for the new $S U(2)$ anomaly [57]. In fact, the $w_{2}(T M) w_{3}(T M)$ topological term plays an important role as the only possible anomaly of an interacting Spin(10) chiral fermion theory-which is responsible for the anomaly-free of the $\mathrm{SO}(10)$ grand unification $[57,73]$.
}

time-reversal $\mathbb{Z}_{2}^{T}$ transformation, Ref. [5] detects the $\mathcal{T} B B$ anomaly, which is linear in $\mathbb{Z}_{2}^{T}$ transformation $\mathcal{T}$ and quadratic to the two-cochain $B$.

(ii) In Sec. II B, we have derived the anomaly by first turning on the two-form gauge field $B$, and further place the theory on an unorientable manifold. We find that to make sense of the $4 \mathrm{~d}$ theta term on an unorientable manifold, we need to promote the original $4 \mathrm{~d}$ YM theory to a combined $4 \mathrm{~d}-5 \mathrm{~d}$ system. The $5 \mathrm{~d}$ theory is an invertible TQFT. In the following, we reverse the logic:

(Step 1) We first formulate the $\mathrm{SU}(2) \mathrm{YM}$ on an unorientable manifold before activating $B$.

(Step 2) We further match the noninvariance of the $4 d$ $\mathrm{SU}(2)_{\theta=\pi}$ YM theory Eq. (2.37) under $B \rightarrow B+\delta \lambda$ with the noninvariance of $\mathbf{S}_{\text {anom }}$ in Eq. (2.32).

(Step 1) We first place the SU(2) Yang-Mills theory on an unorientable manifold without activating the background field $B$. If we limit to the case that the gauge bundle constraint Eq. (2.25) as $c_{1}\left(V_{\mathrm{U}(2)}\right)=0 \bmod 2$, then the theta term is simplified to

$$
-\pi \int_{M^{4}} c_{2}\left(V_{\mathrm{U}(2)}\right)
$$

which is a well-defined $4 \mathrm{~d}$ term. If we further change the time-reversal property (i.e., Kramers singlet/ doublet) and the statistics (i.e., bosonic/fermionic) of the $S U(2)$ gauge charge by modifying the gauge bundle constraint to $c_{1}\left(V_{\mathrm{U}(2)}\right)=K_{1} w_{1}(T M)^{2}+$ $K_{2} w_{2}(T M) \bmod 2$, the theta term is

$\pi \int_{M^{4}}\left(-c_{2}\left(V_{\mathrm{U}(2)}\right)+\frac{1}{2} c_{1}\left(V_{\mathrm{U}(2)}\right) \cup c_{1}\left(V_{\mathrm{U}(2)}\right)\right)$.

The second term does not make sense for $M^{4}$ unorientable, and one needs to define it by promoting the integral to a $5 \mathrm{~d}$ unorientable manifold $M^{5}$. Following the discussion around Eq. (2.26), the $\mathbb{Z}$-valued cohomology class $c_{1}\left(V_{\mathrm{U}(2)}\right)$ is extended to a $\mathbb{Z}_{2}$ cohomology class $\tilde{c}_{1}\left(V_{\mathrm{U}(2)}\right)$, along with the gauge bundle constraint, $\tilde{c}_{1}\left(V_{\mathrm{U}(2)}\right)=K_{1} w_{1}(T M)^{2}+K_{2} w_{2}(T M) \bmod 2$. Then, Eq. (2.43) shall be reinterpreted as

$$
-\pi \int_{M^{4}} c_{2}\left(V_{\mathrm{U}(2)}\right)+\pi \int_{M^{5}} \frac{1}{2} \delta\left(\tilde{c}_{1}\left(V_{\mathrm{U}(2)}\right) \cup \tilde{c}_{1}\left(V_{\mathrm{U}(2)}\right)\right) .
$$


When $M^{5}$ does not have a boundary, $\pi \int_{M^{5}} \frac{1}{2} \delta \times$ $\left(\tilde{c}_{1}\left(V_{\mathrm{U}(2)}\right) \cup \tilde{c}_{1}\left(V_{\mathrm{U}(2)}\right)\right)$ vanishes. This means that, for a fixed $M^{4}$, the second term in Eq. (2.44) does not depend on the choice of $M^{5}$. Hence, when $B$ is turned off, there is no anomaly for generic $\left(K_{1}, K_{2}\right)$. To summarize, there is no pure time-reversal anomaly of SU(2) YangMills with $\theta=\pi$.

(Step 2) We further turn on the background field $B$. Under the gauge transformation $B \rightarrow B+\delta \lambda$ where $\lambda$ is a $\mathbb{Z}_{2}$-valued one-cochain, the $\mathrm{U}(2)$ field strength $\hat{F}$ transforms as

$$
\hat{F} \rightarrow \hat{F}+\pi \delta \lambda I \text {. }
$$

Using Eq. (2.21), we determine that

$$
\begin{aligned}
& c_{1}\left(V_{\mathrm{U}(2)}\right) \rightarrow c_{1}\left(V_{\mathrm{U}(2)}\right)+\delta \lambda, \\
& c_{2}\left(V_{\mathrm{U}(2)}\right) \rightarrow c_{2}\left(V_{\mathrm{U}(2)}\right)+\frac{1}{2} c_{1}\left(V_{\mathrm{U}(2)}\right) \cup \delta \lambda+\frac{1}{4} \mathcal{P}(\delta \lambda) .
\end{aligned}
$$

The only $4 \mathrm{~d}$ term in Eq. (2.24) is the first term proportional to $c_{2}\left(V_{\mathrm{U}(2)}\right)$. Under $B \rightarrow B+\delta \lambda$,

$$
\begin{aligned}
-\pi \int_{M^{4}} c_{2} \rightarrow & -\pi \int_{M^{4}}\left(c_{2}+\frac{1}{2} c_{1}\left(V_{\mathrm{U}(2)}\right) \cup \delta \lambda+\frac{1}{4} \mathcal{P}(\delta \lambda)\right) \\
= & -\pi \int_{M^{4}} c_{2}-\pi \int_{M^{5}} \delta\left(\frac{1}{2} \tilde{c}_{1}\left(V_{\mathrm{U}(2)}\right) \cup \delta \lambda+\frac{1}{4} \mathcal{P}(\delta \lambda)\right) \\
= & -\pi \int_{M^{4}} c_{2}-\pi \int_{M^{5}} \mathrm{Sq}^{1} \tilde{c}_{1} \cup \delta \lambda+\tilde{c}_{1} \cup \mathrm{Sq}^{1} \delta \lambda+\delta \lambda \cup \mathrm{Sq}^{1} \delta \lambda+\mathrm{Sq}^{2} \mathrm{Sq}^{1} \delta \lambda \\
= & -\pi \int_{M^{4}} c_{2}-\pi \int_{M^{5}}\left[\left(\mathrm{Sq}^{1} B+K_{2} \mathrm{Sq}^{1} w_{2}(T M)\right) \delta \lambda+\left(B+K_{1} w_{1}(T M)^{2}+K_{2} w_{2}(T M)\right) \mathrm{Sq}^{1} \delta \lambda\right. \\
& \left.+\delta \lambda \mathrm{Sq}^{1} \delta \lambda+\mathrm{Sq}^{2} \mathrm{Sq}^{1} \delta \lambda\right] .
\end{aligned}
$$

In the second equality, we replaced $\delta / 2$ by $\mathrm{Sq}^{1}$, which is valid for $\mathbb{Z}_{2}$-valued cocycles, and used the identity $\frac{\delta \mathcal{P}(\delta \lambda)}{4}=$ $\delta \lambda \mathrm{Sq}^{1} \delta \lambda+\mathrm{Sq}^{2} \mathrm{Sq}^{1} \delta \lambda$ since $\delta \lambda$ is a cocycle [8]. On the other hand, the variation of the bulk invertible TQFT $\mathbf{S}_{\text {anom }}$, i.e., the $5 \mathrm{~d}$ integral in Eq. (2.24), is

$$
\begin{aligned}
\mathbf{S}_{\mathrm{anom}} \equiv & \pi \int_{M^{5}} B \mathrm{Sq}^{1} B+\mathrm{Sq}^{2} \mathrm{Sq}^{1} B+K_{1} w_{1}(T M)^{2} \cup \mathrm{Sq}^{1} B+K_{2} \mathrm{Sq}^{1} w_{2}(T M) \cup B+K_{2} w_{2}(T M) \cup \mathrm{Sq}^{1} B \\
= & \pi \int_{M^{5}} \frac{1}{4} \delta \mathcal{P}(B)+K_{1} w_{1}(T M)^{2} \cup \mathrm{Sq}^{1} B+K_{2} \mathrm{Sq}^{1} w_{2}(T M) \cup B+K_{2} w_{2}(T M) \cup \mathrm{Sq}^{1} B \\
\rightarrow & \mathbf{S}_{\mathrm{anom}}+\pi \int_{M^{5}} \frac{1}{4} \delta \mathcal{P}(\delta \lambda)+\frac{1}{2} \delta(B \delta \lambda)+K_{1} w_{1}(T M)^{2} \mathrm{Sq}^{1} \delta \lambda+K_{2} \mathrm{Sq}^{1} w_{2}(T M) \delta \lambda+K_{2} w_{2}(T M) \mathrm{Sq}^{1} \delta \lambda \\
= & \mathbf{S}_{\mathrm{anom}}+\pi \int_{M^{5}} \delta \lambda \mathrm{Sq}^{1} \delta \lambda+\mathrm{Sq}^{2} \mathrm{Sq}^{1} \delta \lambda+\mathrm{Sq}^{1} B \delta \lambda+B \mathrm{Sq}^{1} \delta \lambda \\
& +K_{1} w_{1}(T M)^{2} \mathrm{Sq}^{1} \delta \lambda+K_{2} \mathrm{Sq}^{1} w_{2}(T M) \delta \lambda+K_{2} w_{2}(T M) \mathrm{Sq}^{1} \delta \lambda .
\end{aligned}
$$

In the second equality, we used the identity $\frac{\delta \mathcal{P}(B)}{4}=B \mathrm{Sq}^{1} B+\mathrm{Sq}^{2} \mathrm{Sq}^{1} B$ since $B$ is a cocycle [8], and the formula $\mathcal{P}(B+\delta \lambda)=\mathcal{P}(B)+\mathcal{P}(\delta \lambda)+2 B \delta \lambda$ since $B$ and $\delta \lambda$ are both cocycles. In the third equality, we replaced $\delta / 2$ by $\mathrm{Sq}^{1}$, which is valid for $\mathbb{Z}_{2}$-valued cocycles, and used the identity $\frac{\delta \mathcal{P}(\delta \lambda)}{4}=\delta \lambda \mathrm{Sq}^{1} \delta \lambda+\mathrm{Sq}^{2} \mathrm{Sq}^{1} \delta \lambda$ since $\delta \lambda$ is a cocycle [8].

Comparing Eq. (2.46) and Eq. (2.47), we find that the noninvariance of the $4 \mathrm{~d}$ terms Eq. (2.46) precisely cancels the noninvariance of the $5 \mathrm{~d}$ terms Eq. (2.47). Thus the combined $4 \mathrm{~d}-5 \mathrm{~d}$ coupled system $-\pi \int_{M^{4}} c_{2}\left(V_{\mathrm{U}(2)}\right)+\mathbf{S}_{\text {anom }}$ is symmetric under the background gauge transformation of $B$, and thus is anomaly-free under the one-form background gauge transformation. ${ }^{27}$ Furthermore, since both the boundary theory Eq. (2.42) and the bulk invertible TQFT $\mathbf{S}_{\text {anom }}$ are well defined on unorientable manifold $M^{4}$ and $M^{5}$, respectively, the full system $\pi \int_{M^{4}} c_{2}\left(V_{\mathrm{U}(2)}\right)+\mathbf{S}_{\text {anom }}$ also respects the time-reversal symmetry. Thus we again arrive at the conclusion that the combined partition function Eq. (2.36) is well defined and free of the 't Hooft anomalies of both one-form symmetry, time-reversal symmetry, and their mixed anomaly.

\footnotetext{
${ }^{27}$ On an unorientable manifold, the mixed time-reversal and one-form anomaly reduces to the one-form anomaly, since time-reversal symmetry is "gauged" on an unorientable manifold and it is too late to break $\mathbb{Z}_{2}^{T}$.
} 


\section{Topological term on torsion-free orientable manifolds}

In the previous Secs. II B and II C, we derived the mixed anomaly by first reformulating the theta term in terms of characteristic classes, and then we make sense of it on unorientable manifolds by promoting the ill-defined terms on five manifolds. However, there is a loophole: Eq. (2.23) is not well defined even on an oriented manifold, because $c_{1}\left(V_{\mathrm{U}(2)}\right) \cup B$ and $\frac{1}{2} \mathcal{P}(B)$, as a $\mathbb{Z}_{2}$ and a $\mathbb{Z}_{4}$ valued cohomology, respectively, are ill -defined when the coefficients are fractional. In this subsection, we resolve this issue, for certain manifolds, by lifting the $\mathbb{Z}_{2}$ class $B$ to a $\mathbb{Z}$ class $\tilde{B}$, i.e.,

$$
B=\tilde{B} \bmod 2 .
$$

Here we restrict to the orientable manifolds $M^{4}$ with torsionfree cohomology class $H_{1}\left(M^{4}, \mathbb{Z}\right)$ [74] where the lifting makes sense. Hence Eq. (2.23) becomes

$$
\begin{gathered}
\pi \int_{M^{4}}-c_{2}\left(V_{\mathrm{U}(2)}\right)+\frac{c_{1}\left(V_{\mathrm{U}(2)}\right) \cup c_{1}\left(V_{\mathrm{U}(2)}\right)}{2} \\
-\frac{1}{2} c_{1}\left(V_{\mathrm{U}(2)}\right) \cup \tilde{B}+\frac{\tilde{B} \cup \tilde{B}}{4} .
\end{gathered}
$$

To further formulate Eq. (2.49) on an unorientable manifold, we note that every unorientable manifold $M$ contains nontrivial torsion in $H_{1}(M, \mathbb{Z})$, and thus the lifting does not exist. This implies that on an unorientable manifold $M^{4}$ and $M^{5}$, it is not possible to promote a $\mathbb{Z}_{2}$ cohomology class to a $\mathbb{Z}$ cohomology class. However, the derivation of the $5 \mathrm{~d}$ anomaly polynomial Eq. (2.32) still goes through.

\section{E. Consequences and interpretations of four siblings of "anomalies"}

In this section, we discuss the two siblings of anomalies labeled by $\left(K_{1}=0, K_{2}\right)$ and $\left(K_{1}=1, K_{2}\right)$. We also compare our results with the known mixed $\mathbb{Z}_{2,[1]}^{e}-\mathbb{Z}_{2}^{T}$ anomaly discussed in [5].

(1) When $\left(K_{1}, K_{2}\right)=(0,0)$, the bulk anomaly polynomial is

$$
\pi \int_{M^{5}} B \mathrm{Sq}^{1} B+\mathrm{Sq}^{2} \mathrm{Sq}^{1} B=\frac{\pi}{2} \int_{M^{5}} \tilde{w}_{1}(T M) \cup \mathcal{P}(B),
$$

which is nonvanishing only on an unorientable $M^{5}$. This equality has been explored in Ref. [8] in relating to the 4d YM theory's anomaly. Furthermore, we find that this equality is also explained in a remarkable mathematical note in Ref. [75].

Below let us gain a better understanding based on Ref. [75]: Let $\mathbb{Z}_{w_{1}}$ be the orientation local system, and then $H^{1}\left(\mathrm{BO}(1), \mathbb{Z}_{w_{1}}\right)=\mathbb{Z}_{2}$. Indeed, this is the group cohomology $H^{1}\left(\mathbb{Z}_{2}, \mathbb{Z}_{\sigma}\right)$, where $\mathbb{Z}_{\sigma}$ denotes $\mathbb{Z}$ with the sign action. The pullback of the nonzero element of $H^{1}\left(\mathrm{BO}(1), \mathbb{Z}_{w_{1}}\right)$ under the map $M \rightarrow \mathrm{B} \mathbb{Z}_{2}$ determined by $w_{1}(T M) \in H^{1}\left(M, \mathbb{Z}_{2}\right)$ is called the twisted first Stiefel-Whitney class $\tilde{w}_{1} \in H^{1}\left(M, \mathbb{Z}_{w_{1}}\right)$. Its mod 2 reduction is the usual first Stiefel-Whitney class in an untwisted $\mathbb{Z}_{2}$ cohomology. We consider its reduction $\tilde{w}_{1} \in H^{1}\left(M,\left(\mathbb{Z}_{4}\right)_{w_{1}}\right)$ in a twisted $\bmod 4$ cohomology. Here $\mathcal{P}$ denotes the Pontryagin square $\mathcal{P}: H^{2}\left(M, \mathbb{Z}_{2}\right) \rightarrow H^{4}\left(M, \mathbb{Z}_{4}\right)$. In Eq. (2.50), we use cup and cap products in twisted $\mathbb{Z}_{4}$ cohomology: if $[M]$ denotes the fundamental class in the twisted $\mathbb{Z}_{4}$ cohomology, this means that

$$
\begin{aligned}
H^{1}\left(M,\left(\mathbb{Z}_{4}\right)_{w_{1}}\right) & \otimes H^{4}\left(M, \mathbb{Z}_{4}\right) \stackrel{\cup}{\longrightarrow} H^{5}\left(M,\left(\mathbb{Z}_{4}\right)_{w_{1}}\right) \\
& \stackrel{\cap[M]}{\longrightarrow} \mathbb{Z}_{4} .
\end{aligned}
$$

However, since $2 \tilde{w}_{1}$ is a twisted coboundary, $2\left\langle\tilde{w}_{1} \cup \mathcal{P}(B),[M]\right\rangle=0 \bmod 4,\left\langle\tilde{w}_{1} \cup \mathcal{P}(B),[M]\right\rangle$ is even, hence it makes sense to divide by 2 and obtain an element of $\mathbb{Z}_{2}$. This defines $\frac{1}{2} \tilde{w}_{1}(T M) \cup \mathcal{P}(B)$ as a mod 2 class in the fifth cohomology group $H^{5}(\mathrm{BO} \times$ $\left.\mathrm{B}^{2} \mathbb{Z}_{2}, \mathrm{U}(1)\right)$ which is also a bordism invariant of the fifth bordism group $\Omega_{5}^{\mathrm{O}}\left(\mathrm{B}^{2} \mathbb{Z}_{2}\right)$.

There are two options for the boundary $M^{4}$ : orientable or unorientable.

(a) When $M^{4}$ is orientable, the time reversal of the $\mathrm{SU}(2)_{\theta=\pi}$ theory is not gauged. However, there is still a way to probe the mixed $\mathbb{Z}_{2,[1]}^{e}-\mathbb{Z}_{2}^{T}$ anomaly, following the approach of [5]. We first couple the $\mathrm{SU}(2)_{\theta=\pi}$ Yang-Mills to background gauge field $B$, and then perform a global time-reversal transformation. To determine how the theta term changes under time reversal, we make use of the fact that shifting $\theta$ by $2 \pi$ amounts to changing the parameter $p$ of the counterterm by 1 , where the counterterm is $2 \pi \frac{p}{4} \int_{M^{4}} \mathcal{P}(B)$ and $p \in \mathbb{Z}_{4}$, i.e.,

$$
(\theta+2 \pi, p) \leftrightarrow(\theta, p+1) .
$$

Under time reversal, both the theta term Eq. (2.19) and the counterterm change sign, i.e., $\mathbb{Z}_{2}^{T}:(\pi, p) \rightarrow(-\pi,-p)$. Using the identification Eq. (2.52), $(-\pi,-p) \leftrightarrow(\pi,-p-1)$,

$$
\mathbb{Z}_{2}^{T}:(\pi, p) \rightarrow(\pi,-p-1) .
$$

Equivalently, under time reversal, the theta term is unchanged, but there is a shift of the counterterm

$$
\delta \mathbf{S}_{E}\left[M^{4}\right]=-\frac{\pi(2 p+1)}{2} \int_{M^{4}} \mathcal{P}(B) .
$$

The noninvariance in Eq. (2.54) cannot be zero by properly choosing $p \in \mathbb{Z}$, which represents an anomaly. The anomaly Eq. (2.54) can be canceled by the 't Hooft anomaly inflow Eq. (2.50). 
So it is important to emphasize that the $4 \mathrm{~d}$ anomaly from $\mathcal{T} B B$ detected by [5] (and Sec. 2 of Ref. [8]) is precisely captured by the bordism invariant $\frac{1}{2} \tilde{w}_{1}(T M) \cup \mathcal{P}(B)$ in Eq. (2.50) noticed in Ref. [8].

(b) When $M^{4}$ is unorientable, the anomaly can be detected as well, as discussed in Secs. II B and II C.

(2) When $\left(K_{1}, K_{2}\right)=(1,0)$, the bulk action is

$$
\pi \int_{M^{5}} B \mathrm{Sq}^{1} B+\mathrm{Sq}^{2} \mathrm{Sq}^{1} B+w_{1}(T M)^{2} \mathrm{Sq}^{1} B
$$

which is nonvanishing only when $M^{5}$ is unorientable.

(a) When $M^{4}$ is orientable, one cannot probe $K_{1}$. This is because for $\int_{M^{5}} w_{1}(T M)^{2} \mathrm{Sq}^{1} B$ to be nonvanishing mod 2 on $M^{5}$, there should be at least two or more orientation reversing cycles in $M^{5}$, and hence there should be at least one orientation cycle in $M^{4}$. Thus if $M^{4}$ is orientable, even if $M^{5}$ is unorientable, we still cannot detect a particular $4 \mathrm{~d}$ anomaly associated with the $5 \mathrm{~d}$ term $K_{1} w_{1}(T M)^{2} \mathrm{Sq}^{1} B$.

(b) When $M^{4}$ is unorientable, the anomaly can be detected, as discussed in Secs. II B and II C.

(3) When $\left(K_{1}, K_{2}\right)=(0,1)$ :

(a) If $M^{5}$ is a closed $5 \mathrm{~d}$ manifold (regardless whether orientable or unorientable), we cannot detect the term $\int_{M^{5}} K_{2} \mathrm{Sq}^{1}\left(w_{2}(T M) \cup B\right)$.

(b) If $M^{5}$ is a $5 \mathrm{~d}$ manifold with a $4 \mathrm{~d}$ boundary $M^{4}$ (regardless orientable or unorientable in $5 \mathrm{~d}$ or in 4d) and $w_{2}(T M)$ is nontrivial on both $M^{4}$ and $M^{5}$ (e.g., non-Pin ${ }^{+}$manifolds), we can detect the term $\int_{M^{5}} K_{2} \mathrm{Sq}^{1}\left(w_{2}(T M) \cup B\right)$ on the $4 \mathrm{~d}$ boundary via the one-form background gauge transformation. On an $M^{5}$ with a boundary $M^{4}$, we regard $\int_{M^{5}} K_{2} \mathrm{Sq}^{1}\left(w_{2}(T M) \cup B\right)$ schematically as $4 \mathrm{~d}$ fractional SPTs, which is characterized by a 4d ill-defined term with a fractional coefficient $\int_{M^{4}} K_{2} \frac{1}{2}\left(w_{2}(T M) \cup B\right)$. Two copies of such $4 \mathrm{~d}$ fractional SPTs become a well-defined time-reversal $\mathbb{Z}_{2}^{T}$ and one-form $\mathbb{Z}_{2,[1]}^{e}$ symmetric $4 \mathrm{~d}$ SPTs/bordism invariant $\int_{M^{5}}\left(w_{2}(T M) \cup B\right)$, with respect to a nontrivial $\mathbb{Z}_{2}$ generator in $\Omega_{4}^{\mathrm{O}}\left(\mathrm{B}^{2} \mathbb{Z}_{2}\right)=\mathbb{Z}_{2}^{4}$; see Ref. [8] and Appendix. Thus, four layers of such $4 \mathrm{~d}$ fractional SPTs become trivial SPTs with respect to $\Omega_{4}^{\mathrm{O}}\left(\mathrm{B}^{2} \mathbb{Z}_{2}\right)$.

The $\int_{M^{5}} \mathrm{Sq}^{1}\left(w_{2}(T M) B\right)$ is similar to the Wess-Zumino-Witten term $[67,68]$ in some way but with its own exoticness:

(i) The familiar WZW term is an integer $\mathbb{Z}$ class $[67,68]$, and here this $\int_{M^{5}} \operatorname{Sq}^{1}\left(w_{2}(T M) B\right)$ has a fractional discrete class. [In some sense, $\int_{M^{5}} \mathrm{Sq}^{1}\left(w_{2}(T M) B\right)$ seems to be a unit generator in $\mathbb{Z}_{4}$ with respect to $4 \mathrm{~d}$ trivial SPTs.]

(ii) The familiar WZW term is written as a path integral of dynamical fields, but here $\int_{M^{5}} \mathrm{Sq}^{1} \times$ $\left(w_{2}(T M) B\right)$ depends on the background probe fields $w_{2}(T M)$ and $B$.

(iii) Both WZW and $\int_{M^{5}} \mathrm{Sq}^{1}\left(w_{2}(T M) B\right)$ govern the $4 \mathrm{~d}$ physics, but they need to be written in one extra higher dimension. It is tempting to speculate that $\int_{M^{5}} \mathrm{Sq}^{1}\left(w_{2}(T M) B\right)$ may be a nonlocal counterterm on $M^{4}$, which is $4 \mathrm{~d}$ in nature but cannot be written in $4 \mathrm{~d}$ alone. The $\int_{M^{5}} \mathrm{Sq}^{1}\left(w_{2}(T M) B\right)$ can access the $5 \mathrm{~d}$ extra bulk, but it does not depend on how $M^{5}$ is chosen as long as $\partial M^{5}=M^{4}$.

Related interpretations and facts about $\int_{M^{5}} K_{2} \mathrm{Sq}^{1} \times\left(w_{2}(T M) \cup B\right)$ are also summarized in Sec. IB.

(4) When $\left(K_{1}, K_{2}\right)=(1,1)$, the interpretation is simply the linear combination of $\left(K_{1}, K_{2}\right)=(1,0)$ and $\left(K_{1}, K_{2}\right)=(0,1)$ interpretations above.

We will further comment about the fate of the dynamics of four siblings of SU(2) YM based on their "anomalies" Eq. (2.38), in Sec. VIII and in Sec. IX.

\section{F. 5d SPTs/bordism invariants whose boundary allows $4 \mathrm{~d} \operatorname{SU}(2)_{\theta=\pi} \mathrm{YM}$}

\section{On a closed manifold}

We now give various equivalent formulas of the $5 \mathrm{~d}$ SPTs/bordism invariant in Eq. (2.38) on a closed five manifold $M_{\text {closed }}^{5}$ :

$$
\begin{aligned}
\mathbf{Z}_{\mathrm{SPT}_{\left(K_{1}, K_{2}\right)}}^{5 \mathrm{~d}}\left[M_{\text {closed }}^{5}\right] & =\exp \left(\mathrm{i} \pi \int_{M^{5}} B \mathrm{Sq}^{1} B+\mathrm{Sq}^{2} \operatorname{Sq}^{1} B+K_{1} w_{1}(T M)^{2} \operatorname{Sq}^{1} B+K_{2} \operatorname{Sq}^{1}\left(w_{2}(T M) B\right)\right) \\
& =\exp \left(\mathrm{i} \pi \int_{M^{5}} B \mathrm{Sq}^{1} B+\mathrm{Sq}^{2} \mathrm{Sq}^{1} B+K_{1} w_{1}(T M)^{2} \mathrm{Sq}^{1} B\right) \\
& =\exp \left(\mathrm{i} \pi \int_{M^{5}} B \mathrm{Sq}^{1} B+\left(w_{2}(T M)+w_{1}(T M)^{2}\right) \mathrm{Sq}^{1} B+K_{1} w_{1}(T M)^{2} \mathrm{Sq}^{1} B\right) \\
& =\exp \left(\mathrm{i} \pi \int_{M^{5}} B \operatorname{Sq}^{1} B+w_{3}(T M) B+\left(1+K_{1}\right) w_{1}(T M)^{3} B\right)
\end{aligned}
$$




$$
\begin{gathered}
=\exp \left(\mathrm{i} \pi \int_{M^{5}} \frac{1}{2} \tilde{w}_{1}(T M) \cup \mathcal{P}(B)+K_{1} w_{1}(T M)^{3} B\right) \\
=\exp \left(\mathrm{i} \pi \int_{M^{5}} \frac{1}{4} \delta\left(\mathcal{P}_{2}\left(B_{2}\right)\right)+K_{1} w_{1}(T M)^{3} B\right) .
\end{gathered}
$$

In the second line, we knew already from the derivation of Eq. (2.33) that $\exp \left(\mathrm{i} \pi \int_{M^{5}} K_{2} \operatorname{Sq}^{1}\left(w_{2}(T M) B\right)\right)=1$ on a closed manifold.

In the fourth line, we used $w_{1}(T M)^{2} \mathrm{Sq}^{1} B=\mathrm{Sq}^{1}\left(w 1(T M)^{2} B\right)=w_{1}(T M)\left(w_{1}(T M)^{2} B\right)=w_{1}(T M)^{3} B$ where the second equality uses the $\mathrm{Wu}$ formula on a closed manifold. We also used

$$
\begin{aligned}
u_{3} B & =w_{1}(T M) w_{2}(T M) B=\operatorname{Sq}^{1}\left(w_{2}(T M) B\right)=\left(\operatorname{Sq}^{1} w_{2}(T M)\right) B+w_{2}(T M) \mathrm{Sq}^{1} B \\
& =\left(w_{1}(T M) w_{2}(T M)+w_{3}(T M)\right) B+w_{2}(T M) \mathrm{Sq}^{1} B, \\
& \Rightarrow w_{2}(T M) \mathrm{Sq}^{1} B=w_{3}(T M) B \bmod 2,
\end{aligned}
$$

by the $\mathrm{Wu}$ formula on a closed five manifold. ${ }^{28}$

In the fifth line, Eq. (2.58) is based on Eq. (2.50) and Ref. [8,75].

In the sixth line, Eq. (2.59) is based on Eq. (124) in [8].

\section{On a manifold with a boundary}

We also give various equivalent formulas of the $5 \mathrm{~d}$ SPTs/bordism invariant in Eq. (2.38) on a five manifold $M^{5}$ with a nonempty $4 \mathrm{~d}$ boundary $M^{4}$ :

$$
\begin{aligned}
\mathbf{Z}_{\mathrm{SPT}_{\left(K_{1}, K_{2}\right)}}^{\mathrm{d}}\left[M^{5}\right] & =\exp \left(\mathrm{i} \pi \int_{M^{5}} B \operatorname{Sq}^{1} B+\mathrm{Sq}^{2} \operatorname{Sq}^{1} B+K_{1} w_{1}(T M)^{2} \operatorname{Sq}^{1} B+K_{2} \operatorname{Sq}^{1}\left(w_{2}(T M) B\right)\right) \\
& =\exp \left(\mathrm{i} \pi \int_{M^{5}} \frac{1}{2} \tilde{w}_{1}(T M) \cup \mathcal{P}(B)+K_{1} w_{1}(T M)^{2} \operatorname{Sq}^{1} B+K_{2} \operatorname{Sq}^{1}\left(w_{2}(T M) B\right)\right) \\
& =\exp \left(\mathrm{i} \pi \int_{M^{5}} \beta_{(2,4)} \mathcal{P}(B)+K_{1} w_{1}(T M)^{2} \operatorname{Sq}^{1} B+K_{2} \operatorname{Sq}^{1}\left(w_{2}(T M) B\right)\right) \\
& =\exp \left(\mathrm{i} \pi \int_{M^{5}} \frac{1}{4} \delta(\mathcal{P}(B))+K_{1} w_{1}(T M)^{2} \operatorname{Sq}^{1} B+K_{2} \operatorname{Sq}^{1}\left(w_{2}(T M) B\right)\right)
\end{aligned}
$$

In the third line, we followed Ref. [8] to define $\beta_{(n, m)} \equiv H^{*}\left(-, \mathbb{Z}_{m}\right) \rightarrow H^{*+1}\left(-, \mathbb{Z}_{n}\right)$ as the Bockstein homomorphism associated with the extension $\mathbb{Z}_{n} \stackrel{\cdot m}{\rightarrow} \mathbb{Z}_{n m} \rightarrow \mathbb{Z}_{m}$, where $\cdot m$ is the group homomorphism given by multiplication by $m$. We can show that $\beta_{\left(2,2^{n}\right)}=\frac{1}{2^{n}} \delta \bmod 2$ [8]. Using the bordism group data and the identities given in Refs. [8,10], we rewrite the $4 \mathrm{~d}$ higher anomalies and 5d higher-SPTs/bordism invariants/anomaly polynomials.

\section{CLASSIFICATION OF 4d SU(2) $\theta=\pi$ YANG-MILLS THEORIES AND CLASSIFICATION OF 4d TIME- REVERSAL SYMMETRIC BOSONIC/FERMIONIC SU(2)-SPTS}

In this section we explore the physical meaning of the gauge bundle constraint in Eq. (2.25), i.e.,

$$
w_{2}\left(V_{\mathrm{SO}(3)}\right)=B+K_{1} w_{1}(T M)^{2}+K_{2} w_{2}(T M) \bmod 2, \quad K_{1,2} \in \mathbb{Z}_{2},
$$

and discuss their physical consequences.

\footnotetext{
${ }^{28}$ If we consider instead a different $5 \mathrm{~d}$ SPTs/bordism invariant as $K_{3} w_{2}(T M) \mathrm{Sq}^{1} B$, we have the following equalities on a closed five manifold:

$$
\mathbf{Z}_{\mathrm{SPT}_{\left(K_{3}\right)}}^{5 \mathrm{~d}}\left[M_{\text {closed }}^{5}\right]=\exp \left(\mathrm{i} \pi \int_{M^{5}} K_{3} w_{2}(T M) \mathrm{Sq}^{1} B\right)=\exp \left(\mathrm{i} \pi \int_{M^{5}} K_{3} w_{3}(T M) B\right)
$$




\section{A. Kramers singlet/doublet under time-reversal and bosonic/fermionic Wilson line}

Below we provide some physical interpretations of the four siblings of $4 \mathrm{~d} \mathrm{SU}(2) \mathrm{YM}$ theories in terms of the Wilson line properties.

We introduce the standard $4 \mathrm{~d} \mathrm{SU}(2)$ Yang-Mills path integral $\mathbf{Z}_{\mathrm{SU}(2) \mathrm{YM}}^{4 \mathrm{~d}}[B]$ coupled to the background two-form gauge field $B . \mathbf{Z}_{\mathrm{SU}(2) \mathrm{YM}}^{4 \mathrm{~d}}[B]$ is obtained by replacing $F$ with $\hat{F}-B$ in $\mathbf{Z}_{\text {YM }}^{4 \mathrm{~d}}$ in Eq. (1.1). We also need to impose the gauge bundle constraint $w_{2}(E) \equiv w_{2}\left(V_{\mathrm{SO}(3)}\right)=B$, which can be imposed by introducing a Lagrangian multiplier, ${ }^{29}$

$$
\int[D \Lambda] \mathbf{Z}_{\mathrm{SU}(2) \mathrm{YM}}^{4 \mathrm{~d}}[B] \exp \left(\mathrm{i} \pi \int \Lambda \cup\left(w_{2}(E)-B\right)\right) .
$$

(i) Electric two-surface $U_{e}$ : Mathematically, integrating out the Lagrange multiplier $\Lambda$ sets $\left(w_{2}(E)-B\right)=$ $0 \bmod 2$. Physically, $\exp \left(\mathrm{i} \pi \int \Lambda\right)$ plays the role of an electric two-surface $U_{e}=\exp \left(\mathrm{i} \pi \int \Lambda\right)$, which measures one-form $e$-symmetry $\mathbb{Z}_{2,[1]}^{e}$. The magnetic 't Hooft line lives on the boundary of an electric twosurface $U_{e}=\exp \left(\mathrm{i} \pi \int \Lambda\right)$. Since $U_{e}$ is dynamical, the 't Hooft line is not genuine and thus is not in the line spectrum for the $\mathrm{SU}(2)$ gauge theory [6].

(ii) Magnetic two-surface $U_{m}$ is given by $\exp \left(\mathrm{i} \pi \int w_{2} \times\right.$ $(E)$ ). The boundary of $U_{m}$ supports the Wilson loop $W_{e}=\operatorname{Tr}(\mathrm{P} \exp (\mathrm{i} \oint a))$. Unlinking a two-surface $U_{e}$ and a Wilson loop $W_{e}$ yields a nontrivial statistical $\pi$-phase $e^{\mathrm{i} \pi}=-1$.

Following Sec. II, we enrich the gauge bundle constraint as Eq. (2.25) by introducing two couplings labeled by $\left(K_{1}, K_{2}\right)$, and the partition function is

$$
\begin{aligned}
\mathbf{Z}_{\mathrm{SU}(2) \mathrm{YM}_{\left(K_{1}, K_{2}\right)}}^{4 \mathrm{~d}}[B] \equiv & \int[D \Lambda] \mathbf{Z}_{\mathrm{SU}(2) \mathrm{YM}}^{4 \mathrm{~d}}[B] \exp (\mathrm{i} \pi \\
& \times \int \Lambda \cup\left(w_{2}(E)-\left(B+K_{1} w_{1}(T M)^{2}\right.\right. \\
& \left.\left.\left.+K_{2} w_{2}(T M)\right)\right)\right) .
\end{aligned}
$$

As we just deduced, the magnetic two-surface $U_{m} \sim$ $\exp \left(\mathrm{i} \pi \int w_{2}(E)\right)$ has its boundary as Wilson loop $W_{e}=\operatorname{Tr}(\operatorname{P} \exp (\mathrm{i} \oint a))$. We will apply this relation to the four siblings with the YM partition function Eq. (3.1) and its constraint Eq. (2.25) and discuss the properties of the Wilson lines.

\footnotetext{
${ }^{29}$ We can also introduce an additional Pontryagin square $B$ term $\exp \left(\mathrm{i} \frac{\pi}{2} p \mathcal{P}(B)\right)$ with $p \in \mathbb{Z}_{4}$ into the path integral, as the pioneer works Refs. $[6,62]$ do. However, this weight factor term only will result in shifting (thus relabeling) of the classification of $4 \mathrm{~d} \mathrm{SU}(2)_{\theta=\pi}$ theories that we are going to reveal. We use the notations in [8].
}

(1) $\left(K_{1}, K_{2}\right)=(0,0)$ : The gauge bundle constraint is $w_{2}(E)=B \bmod 2$. The magnetic 2-surface $U_{m} \sim$ $\exp \left(\mathbf{i} \pi \int w_{2}(E)\right)$ has no decoration other than the two-form background $B$ field. Thus the one-Wilson line $W_{e}$ (which can live on the magnetic two-surface $U_{m}$ 's boundary) is Kramer singlet $\left(T^{2}=+1\right)$ and bosonic.

(2) $\left(K_{1}, K_{2}\right)=(1,0)$ : The gauge bundle constraint becomes $w_{2}(E)=B+w_{1}(T M)^{2} \bmod 2$. The magnetic two-surface $U_{m} \sim \exp \left(\mathrm{i} \pi \int w_{2}(E)\right)$ has a decoration $\int w_{1}(T M)^{2}$ other than the two-form $B$ field. But $\int w_{1}(T M)^{2}$ is a topological term in a cohomology group $H^{2}\left(\mathbb{Z}_{2}^{T}, \mathrm{U}(1)\right)$ also in bordism group $\Omega_{2}^{\mathrm{O}}(p t)$, which is effectively a $1+1 \mathrm{D}$ Haldane's antiferromagnetic quantum spin-one chain (Haldane chain) protected by time-reversal symmetry. It is well known that there exists twofold degeneracy due to Kramer doublet $\left(T^{2}=-1\right)$ on the boundary of the Haldane chain. Thus due to $\int w_{1}(T M)^{2}$ decoration, the Wilson line $W_{e}$ is Kramer doublet $\left(T^{2}=-1\right)$ and bosonic.

(3) $\left(K_{1}, K_{2}\right)=(0,1)$ : The gauge bundle constraint becomes $w_{2}(E)=B+w_{2}(T M) \bmod 2$. The magnetic two-surface $U_{m} \sim \exp \left(\mathrm{i} \pi \int w_{2}(E)\right)$ has a decoration $\int w_{2}(T M)$ other than the two-form $B$ field. But $\int w_{2}(T M)$ is associated with a spin structure. The $1 \mathrm{~d}$ boundary of the $2 \mathrm{~d} \int w_{2}(T M)$ theory supports a world line of a particle with fermionic statistics. Thus due to $\int w_{2}(T M)$ decoration, the Wilson line $W_{e}$ living on the boundary of the magnetic two-surface $U_{m}$ is fermionic. Since $w_{2}(T M)$ specifies the extension of $O(d)$ by the fermionic-parity $\mathbb{Z}_{2}^{F}$ via the short exact sequence $1 \rightarrow \mathbb{Z}_{2}^{F} \rightarrow \mathrm{Pin}^{+}(d) \rightarrow \mathrm{O}(d) \rightarrow 1$ or equivalently the induced fiber sequence $\mathrm{B} \mathbb{Z}_{2}^{F} \rightarrow$ $\mathrm{BPin}^{+}(d) \rightarrow \mathrm{BO}(d) \stackrel{w_{2}(T M)}{\longrightarrow} \mathrm{B}^{2} \mathbb{Z}_{2}^{F}, w_{2}(T M)$ specifies a projective representation $\operatorname{Pin}^{+}(d)$ of the spacetime symmetry $\mathrm{O}(d)$ [38]. The $\mathrm{Pin}^{+}(d)$ demands the Euclidean reflection $R^{2}=+1$, and thus the Wick rotated time-reversal transformation $\mathcal{T}^{2}=-1$ in the Lorentz signature [40]. Another way to see $\mathcal{T}^{2}=-1$ is to use the methods of symmetry extension and the pullback trivialization [54,57]. Defining the Wilson line operator on the boundary of the magnetic twosurface $U_{m}$ requires a trivialization of $w_{2}(T M)=0$, which amounts to requiring a $\operatorname{Pin}^{+}(d)$ structure. The $\operatorname{Pin}^{+}(d)$ structure imposes $\mathcal{T}^{2}=-1$ and fermionic statistics on the line. In summary, due to the $\int w_{2}(T M)$ decoration, the Wilson line $W_{e}$ is both Kramer singlet $\left(T^{2}=+1\right)$ and fermionic.

(4) $\left(K_{1}, K_{2}\right)=(1,1)$ : The gauge bundle constraint is $w_{2}(E)=B+w_{1}(T M)^{2}+w_{2}(T M) \bmod 2$. Since $w_{2}(T M)$ specifies the extension of $O(d)$ by the fermionic-parity $\mathbb{Z}_{2}^{F}$ via the short exact sequence $1 \rightarrow \mathbb{Z}_{2}^{F} \rightarrow \operatorname{Pin}^{-}(d) \rightarrow \mathrm{O}(d) \rightarrow 1$ or equivalently 
the induced fiber sequence $\mathrm{B}_{2}^{F} \rightarrow \mathrm{BPin}^{-}(d) \rightarrow$ $\mathrm{BO}(d) \stackrel{w_{1}(T M)^{2}+w_{2}(T M)}{\longrightarrow} \mathrm{B}^{2} \mathbb{Z}_{2}^{F}$, so $w_{1}(T M)^{2}+w_{2}(T M)$ specifies a projective representation $\operatorname{Pin}^{-}(d)$ of the spacetime symmetry $\mathrm{O}(d)$ [38]. The $\mathrm{Pin}^{-}(d)$ demands the Euclidean reflection $R^{2}=-1$, and thus the Wick rotated time-reversal transformation $\mathcal{T}^{2}=$ +1 in the Lorentz signature [40]. Another way to see $\mathcal{T}^{2}=-1$ is to use the methods of symmetry extension and the pullback trivialization [54,57]. Defining the Wilson line operator on the boundary of the magnetic two-surface $U_{m}$ requires the trivialization of $w_{1}(T M)^{2}+w_{2}(T M)=0$, which amounts to requiring the $\operatorname{Pin}^{-}(d)$ structure. The $\operatorname{Pin}^{-}(d)$ structure imposes $\mathcal{T}^{2}=+1$ and fermionic statistics on the line. The combined effect of $\int w_{1}(T M)^{2}+$ $w_{2}(T M)$ decoration means that the one-Wilson line $W_{e}$ is a Kramer singlet $\left(T^{2}=+1\right)$ and fermionic. In fact, our discussions above are universally applicable to more general SU(N) YM theories. ${ }^{30}$ This way of enumerating gauge theories (based on new gauge bundle constraints) guides us to obtain new classes of gauge theories beyond the framework of Ref. [62]. The implications are not restricted to merely $4 \mathrm{~d} \mathrm{SU}(2)_{\theta=\pi} \mathrm{YM}$. This phenomenon (also in [38]) can be poetically phrased as Lorentz symmetry fractionalization [76].

\section{B. Enumeration of gauge theories from dynamically gauging 4d SPTs: View from $4 d$ cobordism group data}

We have discussed the four siblings of $\mathrm{SU}(2)_{\theta=\pi} \mathrm{YM}$ theories given by $\mathbf{Z}_{\mathrm{SU}(2) \mathrm{YM}_{\left(K_{1}, K_{2}\right)}^{4 \mathrm{~d}}}[B]$ in Eq. (3.1), with four distinct sets of new anomalies derived in Sec. II, and with Kramer singlet/doublet $\left(T^{2}=+1 /-1\right)$ or bosonic/fermionic Wilson lines in Sec. III A. With these properties shown, we are confident that they are really four distinct classes of $\mathrm{SU}(2)_{\theta=\pi} \mathrm{YM}$ theories (at least at the UV high energy). The two distinct 't Hooft anomalies of $\left(K_{1}, K_{2}\right)$ also shows that $\mathrm{SU}(2)_{\theta=\pi} \mathrm{YM}$ theories with distinct $K_{1}$ are distinct.

In this subsection, we would like to construct and enumerate these four sSiblings of $\mathrm{SU}(2)_{\theta=\pi} \mathrm{YM}$ theories by dynamically gauging the $S U(2)$ symmetry from $4 d$ timereversal symmetric SU(2)-SPTs. To this end, we follow Freed-Hopkins [41] to consider a suitable group extension from the time-reversal symmetry [where the spacetime $d$ manifold requires the orthogonal group $\mathrm{O}(d)$-structure] via a $\mathrm{SU}(2)$ extension:

$$
1 \rightarrow \mathrm{SU}(2) \rightarrow G^{\prime} \rightarrow \mathrm{O}(d) \rightarrow 1 .
$$

These $4 \mathrm{~d}$ SPTs can be regarded as $4 \mathrm{~d}$ co/bordism invariants of

$$
\Omega_{4, \text { tor }}^{G^{\prime}}
$$

\footnotetext{
${ }^{30}$ Related studies along this line of analysis have also appeared in $[38,65,66]$.
}

which is the torsion subgroup $\Omega_{4, \text { tor }}^{G^{\prime}}$ of $\Omega_{4}^{G^{\prime}}$ for all the possible $G^{\prime}$ under the above group extension. The extension is classified by $H^{2}\left(\mathrm{BO}(d), \mathbb{Z}_{2}\right)=\mathbb{Z}_{2} \times \mathbb{Z}_{2}$ for $d>1$, generated by $w_{1}^{2}(T M)$ and $w_{2}(T M)$.

The solution $G^{\prime}$ of this extension problem $1 \rightarrow \mathrm{SU}(2) \rightarrow$ $G^{\prime} \rightarrow \mathrm{O} \rightarrow 1$ is given in [41] with indeed four choices of $G^{\prime}=\mathrm{O} \times \mathrm{SU}(2)$, or $\mathrm{E} \times_{\mathbb{Z}_{2}} \mathrm{SU}(2)$, or $\mathrm{Pin}^{+} \times_{\mathbb{Z}_{2}} \mathrm{SU}(2)$, or $\mathrm{Pin}^{-} \times_{\mathbb{Z}_{2}} \mathrm{SU}(2){ }^{31}$

Following the similar study in Ref. [38], there is a correspondence between the element $\mathrm{b}=K_{1} w_{1}(T M)^{2}+$ $K_{2} w_{2}(T M)$ and $H^{2}\left(\mathrm{BO}(d), \mathbb{Z}_{2}\right)=\left(\mathbb{Z}_{2}\right)^{2}$. It will soon become clear that $\mathrm{b}$ is related to $w_{2}\left(V_{\mathrm{SO}(3)}\right)-B$ (i.e., the difference of the gauge bundle $E=V_{\mathrm{SO}(3)}$ connection and the background gauge connection $B$ ). Then the four central extension choices labeled by $\mathrm{b}$ are the following:

(1) $\mathrm{b}=0 \Rightarrow G^{\prime}=\mathrm{O}(d) \times \mathrm{SU}(2) \Rightarrow$ After gauging $\mathrm{SU}(2)$, we gain the gauge bundle constraint with $K_{1}=K_{2}=0$,

$$
w_{2}\left(V_{\mathrm{SO}(3)}\right)-B=0 .
$$

We compute the co/bordism group in Table III (details given in Appendix). For $d=4$, we obtain

$$
\Omega_{4, \text { tor }}^{\mathrm{O}(d) \times \mathrm{SU}(2)}=\mathbb{Z}_{2}^{3},
$$

whose bordism invariants are generated by three generators of mod 2 classes:

$$
\left\{\begin{array}{l}
w_{1}(T M)^{4} \\
w_{2}(T M)^{2}, \\
c_{2} \bmod 2
\end{array}\right.
$$

The $c_{2}$ is the second Chern class of the $\mathrm{SU}(2)$ gauge bundle.

(2) $\mathrm{b}=w_{1}(T M)^{2} \Rightarrow G^{\prime}=\mathrm{E}(d) \times_{\mathbb{Z}_{2}} \mathrm{SU}(2)^{32} \Rightarrow$ After gauging $\mathrm{SU}(2)$, we gain the gauge bundle constraint with $K_{1}=1$ and $K_{2}=0$,

$$
w_{2}\left(V_{\mathrm{SO}(3)}\right)-B=w_{1}(T M)^{2} .
$$

\footnotetext{
${ }^{31}$ The notation $G_{1} \times{ }_{N} G_{2}:=\frac{G_{1} \times G_{2}}{N}$ is defined as the product group $G_{1} \times G_{2}$ mod out their $\left(G_{1}\right.$ 's and $G_{2}$ 's) common normal subgroup $N$ [41].

${ }^{32}$ Here $\mathrm{E}(d)$ satisfies the following two short exact sequences:

$$
\left\{\begin{array}{l}
1 \rightarrow \mathbb{Z}_{2} \rightarrow \mathrm{E}(d) \rightarrow \mathrm{O}(d) \rightarrow 1 \\
1 \rightarrow \mathrm{SO}(d) \rightarrow \mathrm{E}(d) \rightarrow \mathbb{Z}_{4} \rightarrow 1
\end{array}\right.
$$

given that we also accept the well-known fact $1 \rightarrow \mathrm{SO}(d) \rightarrow$ $\mathrm{O}(d) \rightarrow \mathbb{Z}_{2}^{T} \rightarrow 1$. Here the above finite groups have physical interpretations: $\mathbb{Z}_{2}=\mathbb{Z}_{2}^{b}$ is a bosonic group, and $\mathbb{Z}_{4}=\mathbb{Z}_{4}^{T b}$ is the extended group under $1 \rightarrow \mathbb{Z}_{2}^{b} \rightarrow \mathbb{Z}_{4}^{T b} \rightarrow \mathbb{Z}_{2}^{T} \rightarrow 1$. Thus $\mathrm{E}(d)=$ $\mathbb{Z}_{2}^{b} \rtimes \mathrm{O}(d)=\mathrm{SO}(d) \rtimes \mathbb{Z}_{4}^{T b}=\left(\mathrm{SO}(d) \times \mathbb{Z}_{2}^{b}\right) \rtimes \mathbb{Z}_{2}^{T}$. Another way to define $\mathrm{E}(d)$ is a specific subgroup of $\mathrm{O}(d) \times \mathbb{Z}_{4}$ given in [41].
} 
TABLE III. Cobordism groups $\operatorname{TP}_{d}(\mathrm{O}(d) \times \mathrm{SU}(2))$ and co/bordism invariants. Here $w_{i}(T M)$ is the $i$ th StiefelWhitney class of the spacetime tangent bundle, $c_{2}$ is the second Chern class of the $\mathrm{SU}(2)$ gauge bundle. Here we set $H$ as the Hopf fibration, the $\mathrm{SU}(2)$ bundles on $\mathbb{R P}^{4}, \mathbb{C P}^{2}$, and $\mathrm{SU}(3) / \mathrm{SO}(3)$ are trivial. See also Appendix.

\begin{tabular}{cccc}
\hline \hline$d$ & $\mathrm{TP}_{d, \text { tor }}(\mathrm{O}(d) \times \mathrm{SU}(2))$ & $\mathrm{Co} /$ bordism invariants & Manifold generators $\left(M, V_{\mathrm{SO}(3)}\right)$ \\
\hline 4 & $\mathbb{Z}_{2}^{3}$ & $w_{1}(T M)^{4}, w_{2}(T M)^{2}, c_{2} \bmod 2$ & $\mathbb{R P}^{4}, \mathbb{C P}^{2},\left(S^{4}, H\right)$ \\
5 & $\mathbb{Z}_{2}$ & $w_{2}(T M) w_{3}(T M)$ & $\mathrm{SU}(3) / \mathrm{SO}(3)$ \\
\hline \hline
\end{tabular}

TABLE IV. Cobordism groups $\operatorname{TP}_{d}\left(\mathrm{E}(d) \times_{\mathbb{Z}_{2}} \mathrm{SU}(2)\right)$ and cobordism invariants. Here $w_{i}(T M)$ is the $i$ th StiefelWhitney class of the spacetime tangent bundle, $c_{2}$ is the second Chern class of the U(2) gauge bundle (see footnote 32). The second component in manifold generators $\left(M, V_{\mathrm{SO}(3)}\right)$ is the $\mathrm{SO}(3)$ gauge bundle over the first component. $L_{\mathbb{R}}$ is the real tautological line bundle. $H$ is induced from the Hopf fibration by $\mathrm{SU}(2) \rightarrow \mathrm{SO}(3)$. The $\mathrm{SO}(3)$ bundle on $\mathrm{SU}(3) / \mathrm{SO}(3)$ is trivial. See also Appendix.

\begin{tabular}{cccc}
\hline \hline$d$ & $\mathrm{TP}_{d, \text { tor }}\left(\mathrm{E}(d) \times_{\mathbb{Z}_{2}} \mathrm{SU}(2)\right)$ & Cobordism invariants & Manifold generators $\left(M, V_{\mathrm{SO}(3)}\right)$ \\
\hline 4 & $\mathbb{Z}_{2}^{3}$ & $w_{1}(T M)^{4}, w_{2}(T M)^{2}, c_{2} \bmod 2^{32}$ & $\left(\mathbb{R P}^{4}, 2 L_{\mathbb{R}}+1\right),\left(\mathbb{C P}^{2}, 3\right),\left(S^{4}, H\right)$ \\
5 & $\mathbb{Z}_{2}$ & $w_{2}(T M) w_{3}(T M)$ & $\mathrm{SU}(3) / \mathrm{SO}(3)$ \\
\hline \hline
\end{tabular}

We compute the co/bordism group in Table IV (details given in Appendix). For $d=4$, we obtain

$$
\Omega_{4, \text { tor }}^{\mathrm{E}(d) \times_{\mathbb{Z}_{2}} \mathrm{SU}(2)}=\mathbb{Z}_{2}^{3},
$$

whose bordism invariants are generated by three generators of the mod 2 class:

$$
\left\{\begin{array}{l}
w_{1}(T M)^{4} \\
w_{2}(T M)^{2} \\
c_{2} \bmod 2
\end{array}\right.
$$

$\mathrm{E}(d)$ is defined in [41] which is a subgroup of $\mathrm{O}(d) \times \mathbb{Z}_{4}$, described by two data $(M, j) \in$ $\left(\mathrm{O}(d), \mathbb{Z}_{4}\right)$ such that the $\operatorname{det} M=j^{2}$.

By a different but more physical understanding (see footnote 31), we can further obtain that

$$
\begin{aligned}
\mathrm{E}(d) & =\mathbb{Z}_{2}^{b} \rtimes \mathrm{O}(d)=\mathrm{SO}(d) \rtimes \mathbb{Z}_{4}^{T b} \\
& =\left(\mathrm{SO}(d) \times \mathbb{Z}_{2}^{b}\right) \rtimes \mathbb{Z}_{2}^{T},
\end{aligned}
$$

where the bosonic internal symmetry $\mathbb{Z}_{2}^{b}$ and the time reversal $\mathbb{Z}_{2}^{T}$ form the extended group $\mathbb{Z}_{4}^{T b}$ under $1 \rightarrow \mathbb{Z}_{2}^{b} \rightarrow \mathbb{Z}_{4}^{T b} \rightarrow \mathbb{Z}_{2}^{T} \rightarrow 1$

Here the $c_{2}$ is the second Chern class of the $\mathrm{U}(2)$ gauge bundle. ${ }^{33}$

\footnotetext{
${ }^{33}$ Since the constraint $w_{1}(T M)^{2}=w_{2}\left(V_{\mathrm{SO}(3)}\right)$ is satisfied, let $\beta_{2}$ denote the Bockstein homomorphism associated with the extension $\mathbb{Z} \rightarrow \mathbb{Z} \rightarrow \mathbb{Z}_{2}$, and then $W_{3}\left(V_{\mathrm{SO}(3)}\right)=\beta_{2} w_{2}\left(V_{\mathrm{SO}(3)}\right)=$ $\beta_{2} w_{1}(T M)^{2}=\beta_{2} \operatorname{Sq}^{1} w_{1}(T M)=0$ where $W_{3}\left(V_{\mathrm{SO}(3)}\right)$ is the third integral Stiefel-Whitney class of $V_{\mathrm{SO}(3)}$ and we have used the fact that $\beta_{2} \mathrm{Sq}^{1}=0$; hence $V_{\mathrm{SO}(3)}$ lifts to a $\operatorname{Spin}^{c}(3)=\mathrm{U}(2)$ bundle $V_{\mathrm{U}(2)}$, where $c_{2}=c_{2}\left(V_{\mathrm{U}(2)}\right)$ is the second Chern class of $V_{\mathrm{U}(2)}$.
}

(3) $\mathrm{b}=w_{2}(T M) \Rightarrow G^{\prime}=\operatorname{Pin}^{+} \times_{\mathbb{Z}_{2}} \mathrm{SU}(2) \Rightarrow$ After gauging $\mathrm{SU}(2)$, we gain the gauge bundle constraint with $K_{1}=0$ and $K_{2}=1$,

$$
w_{2}\left(V_{\mathrm{SO}(3)}\right)-B=w_{2}(T M) .
$$

The co/bordism group is computed in $[38,41]$ and in Table V (see also Appendix). For $d=4$, we obtain

$$
\Omega_{4, \text { tor }}^{\text {Pin }^{+} \times_{\mathbb{Z}_{2}} \mathrm{SU}(2)}=\mathbb{Z}_{4} \times \mathbb{Z}_{2},
$$

whose bordism invariants are generated by generators of mod 4 and mod 2 classes:

$$
\left\{\begin{array}{l}
\nu \eta_{\mathrm{SU}(2)}, \quad \text { with a } \nu \in \mathbb{Z}_{4} \text { class }, \\
w_{2}(T M)^{2}
\end{array}\right.
$$

This is related to the interacting version of the CI class topological superconductor in condensed matter physics $[38,41,77]$. Details of these topological terms are discussed in [38].

(4) $\mathrm{b}=w_{2}(T M)+w_{1}(T M)^{2} \Rightarrow G^{\prime}=\mathrm{Pin}^{-} \times_{\mathbb{Z}_{2}} \mathrm{SU}(2) \Rightarrow$ After gauging $\mathrm{SU}(2)$, we gain the gauge bundle constraint with $K_{1}=K_{2}=1$,

$$
w_{2}\left(V_{\mathrm{SO}(3)}\right)-B=w_{2}(T M)+w_{1}(T M)^{2} .
$$

The co/bordism group is computed in $[38,41]$ and in Table VI (see also Appendix). For $d=4$, we obtain

$$
\Omega_{4, \text { tor }}^{\operatorname{Pin}^{-} \times_{\mathbb{Z}_{2}} \mathrm{SU}(2)}=\left(\mathbb{Z}_{2}\right)^{3},
$$

whose bordism invariants are generated by three generators of mod 2 classes: 
TABLE V. Cobordism groups $\mathrm{TP}_{d}\left(\operatorname{Pin}^{+}(d) \times_{\mathbb{Z}_{2}} \mathrm{SU}(2)\right)$ and cobordism invariants. Here $\tilde{w}_{i}$ is the $i$ th StiefelWhitney class of $(T M-n) \otimes V_{\mathrm{SO}(3)}$ where $V_{\mathrm{SO}(3)}$ is the associated vector bundle of the $\mathrm{SO}(3)$ gauge bundle. The $\tilde{w}_{i}$ is computed in Eq. (3.16). The $\eta_{\mathrm{SU}(2)}$ is an eta invariant of the Dirac operator defined in [38]. More details of computation can be read from [38,41]. The second component in manifold generators $\left(M, V_{\mathrm{SO}(3)}\right)$ is the $\mathrm{SO}(3)$ gauge bundle over the first component. $L_{\mathbb{C}}$ is the complex tautological line bundle. The $\mathrm{SO}(3)$ bundle on $\mathrm{SU}(3) / \mathrm{SO}(3)$ is given by the fibration $\mathrm{SO}(3) \rightarrow \mathrm{SU}(3) \rightarrow \mathrm{SU}(3) / \mathrm{SO}(3)$. See also Appendix. Note that [38] actually derives that the $4 \mathrm{~d}$ cobordism invariants are $\tilde{w}_{2}^{2}=w_{2}(T M)^{2}+w_{1}(T M)^{4}$ and $\eta_{\mathrm{SU}(2)}$ with $2 \eta_{\mathrm{SU}(2)}=\tilde{w}_{1} \tilde{w}_{3}=$ $w_{1}(T M) w_{3}(T M)+w_{1}(T M)^{4}$, but since the third Wu class $u_{3}=w_{1}(T M) w_{2}(T M)=0$ on any four manifold, we have $w_{1}(T M) w_{3}(T M)=\mathrm{Sq}^{1}\left(w_{1}(T M) w_{2}(T M)\right)=0$, so by a base change, we can choose the $4 \mathrm{~d}$ cobordism invariants to be $w_{2}(T M)^{2}$ and $\eta_{\mathrm{SU}(2)}$. Also note that the 5d cobordism invariant is actually $\tilde{w}_{2} \tilde{w}_{3}=\left(w_{2}(T M)+\right.$ $\left.w_{1}(T M)^{2}\right)\left(w_{3}(T M)+w_{1}(T M)^{3}\right)$, but since the third Wu class $u_{3}=w_{1}(T M) w_{2}(T M)=0$ on any five manifold, we have $\mathrm{Sq}^{2}\left(w_{1}(T M) w_{2}(T M)\right)=w_{1}(T M) w_{2}(T M)^{2}+w_{1}(T M)^{3} w_{2}(T M)+w_{1}(T M)^{2} w_{3}(T M)=0$; also by the $\mathrm{Wu}$ formula, we have $w_{1}(T M) w_{2}(T M)^{2}=\mathrm{Sq}^{1}\left(w_{2}(T M)^{2}\right)=0$ and $w_{1}(T M)^{5}=\mathrm{Sq}^{1}\left(w_{1}(T M)^{4}\right)=0$ on any five manifold, so $\tilde{w}_{2} \tilde{w}_{3}=w_{2}(T M) w_{3}(T M)$.

\begin{tabular}{cccc}
\hline \hline$d$ & $\mathrm{TP}_{d, \text { tor }}\left(\operatorname{Pin}^{+}(d) \times_{\mathbb{Z}_{2}} \mathrm{SU}(2)\right)$ & Cobordism invariants & Manifold generators $\left(M, V_{\mathrm{SO}(3)}\right)$ \\
\hline 4 & $\mathbb{Z}_{2} \times \mathbb{Z}_{4}$ & $w_{2}(T M)^{2}, \eta_{\mathrm{SU}(2)}$ & $\left(\mathbb{C P}^{2}, L_{\mathbb{C}}+1\right),\left(\mathbb{R} \mathbb{P}^{4}, 3\right)$ \\
5 & $\mathbb{Z}_{2}$ & $w_{2}(T M) w_{3}(T M)$ & $\mathrm{SU}(3) / \mathrm{SO}(3)$ \\
\hline \hline
\end{tabular}

TABLE VI. Cobordism groups $\operatorname{TP}_{d}\left(\operatorname{Pin}^{-}(d) \times_{\mathbb{Z}_{2}} \mathrm{SU}(2)\right)$ and cobordism invariants. Here $\tilde{w}_{i}$ is the $i$ th StiefelWhitney class of $(T M-n) \otimes V_{\mathrm{SO}(3)}$ where $V_{\mathrm{SO}(3)}$ is the associated vector bundle of the $\mathrm{SO}(3)$ gauge bundle. The $\tilde{w}_{i}$ is computed in Eq. (3.16). The $N_{0}^{\prime(4)}$ is the number of the zero modes of the Dirac operator in $4 \mathrm{~d}$. Its value mod 2 is a spin-topological invariant known as the mod 2 index defined as $N_{0}^{\prime} \bmod 2$ in [38]. More details of computation can be read from $[38,41]$. We find that the bordism invariant of $N_{0}^{\prime(4)} \bmod 2$ read from Adams chart has the similar form related to $\tilde{w}_{3} \tilde{\eta}$, where $\tilde{\eta}$ is the eta invariant for the $1 \mathrm{~d}$ Dirac operator, given by the generator of the $1 \mathrm{~d}$ spin bordism group $\Omega_{1, \text { tor }}^{\mathrm{Spin}}(p t)=\mathbb{Z}_{2}$. The $N_{0}^{\prime(5)}$ is the number of the zero modes of the Dirac operator in $5 \mathrm{~d}$. Its value mod 2 is a spin-topological invariant known as the mod 2 index defined in [56,57]. We find that the bordism invariant of $N_{0}^{\prime(5)} \bmod 2$ read from Adams chart has the similar form related to $\tilde{w}_{3}$ Arf, where Arf is an Arf invariant. The second component in manifold generators $\left(M, V_{\mathrm{SO}(3)}\right)$ is the $\mathrm{SO}(3)$ gauge bundle over the first component. $L_{\mathbb{C}}$ is the complex tautological line bundle. $L_{\mathbb{R}}$ is the real tautological line bundle. $H$ is induced from the Hopf fibration by $\mathrm{SU}(2) \rightarrow \mathrm{SO}(3)$. The $\mathrm{SO}(3)$ bundle on $\mathrm{SU}(3) / \mathrm{SO}(3)$ is given by the fibration $\mathrm{SO}(3) \rightarrow \mathrm{SU}(3) \rightarrow \mathrm{SU}(3) / \mathrm{SO}(3)$. The $\mathrm{SO}(3)$ bundle on $S^{1} \times S^{4}$ is induced from the fibration $S^{3} \rightarrow S^{1} \times S^{7} \rightarrow S^{1} \times S^{4}$ by $\mathrm{SU}(2) \rightarrow \mathrm{SO}(3)$. See also Appendix. Note that [38] actually derives that the $4 \mathrm{~d}$ cobordism invariants are $\tilde{w}_{2}^{2}=w_{2}(T M)^{2}+w_{1}(T M)^{4}$, $\tilde{w}_{1}^{4}=w_{1}(T M)^{4}$, and $\left(N_{0}^{\prime(4)} \bmod 2\right)$, and by a base change, we can choose the $4 \mathrm{~d}$ cobordism invariants to be $w_{2}(T M)^{2}, w_{1}(T M)^{4}$, and $\left(N_{0}^{\prime(4)} \bmod 2\right)$. Also note that the $5 \mathrm{~d}$ cobordism invariants are actually $\tilde{w}_{2} \tilde{w}_{3}=\left(w_{2}(T M)+\right.$ $\left.w_{1}(T M)^{2}\right)\left(w_{3}(T M)+w_{1}(T M)^{3}\right)$ and $\left(N_{0}^{\prime(5)} \bmod 2\right)$, but since the third Wu class $u_{3}=w_{1}(T M) w_{2}(T M)=0$ on any five manifold, we have $\mathrm{Sq}^{2}\left(w_{1}(T M) w_{2}(T M)\right)=w_{1}(T M) w_{2}(T M)^{2}+w_{1}(T M)^{3} w_{2}(T M)+w_{1}(T M)^{2} w_{3}(T M)=0$; also by $\mathrm{Wu}$ formula, we have $w_{1}(T M) w_{2}(T M)^{2}=\mathrm{Sq}^{1}\left(w_{2}(T M)^{2}\right)=0$ and $w_{1}(T M)^{5}=\mathrm{Sq}^{1}\left(w_{1}(T M)^{4}\right)=0$ on any five manifold, so $\tilde{w}_{2} \tilde{w}_{3}=w_{2}(T M) w_{3}(T M)$.

\begin{tabular}{cccc}
\hline \hline$d$ & $\mathrm{TP}_{d, \text { tor }}\left(\operatorname{Pin}^{-}(d) \times_{\mathbb{Z}_{2}} \mathrm{SU}(2)\right)$ & Cobordism invariants & Manifold generators $\left(M, V_{\mathrm{SO}(3)}\right)$ \\
\hline 4 & $\mathbb{Z}_{2}^{3}$ & $w_{2}(T M)^{2}, w_{1}(T M)^{4},\left(N_{0}^{\prime(4)} \bmod 2\right)$ & $\left(\mathbb{C P}^{2}, L_{\mathbb{C}}+1\right),\left(\mathbb{R P} P^{4}, 2 L_{\mathbb{R}}+1\right),\left(S^{4}, H\right)$ \\
5 & $\mathbb{Z}_{2}^{2}$ & $w_{2}(T M) w_{3}(T M),\left(N_{0}^{\prime(5)} \bmod 2\right)$ & $\mathrm{SU}(3) / \operatorname{SO}(3), S^{1} \times S^{4}$ \\
\hline \hline
\end{tabular}

$$
\left\{\begin{array}{l}
N_{0}^{\prime} \bmod 2 \\
w_{1}(T M)^{4} \\
w_{2}(T M)^{2}
\end{array}\right.
$$

This is related to the interacting version of the CII class topological insulator in condensed matter physics $[38,41,77]$. Details of these topological terms are discussed in [38].
More information about these (co)bordism group calculations can be read from $[38,41]$. See the appendix of [38] for a quick background review. In particular, since the computation involves no odd torsion, we can use Adams spectral sequence to compute $\Omega_{n}^{G^{\prime}}=\pi_{n}\left(M T G^{\prime}\right)$ :

$$
\operatorname{Ext}_{\mathcal{A}_{2}}^{s, t}\left(\mathrm{H}^{*}\left(M T G^{\prime}, \mathbb{Z}_{2}\right), \mathbb{Z}_{2}\right) \Rightarrow \pi_{t-s}\left(M T G^{\prime}\right)_{2}^{\wedge}
$$

Here $\pi_{t-s}\left(M T G^{\prime}\right)_{2}^{\wedge}$ is the two-completion of the group $\pi_{t-s}\left(M T G^{\prime}\right)$. For example, 


$$
\left\{\begin{array}{l}
M T(\mathrm{O} \times \mathrm{SU}(2))=M \mathrm{O} \wedge \mathrm{BSU}(2)_{+}, \\
M T\left(\mathrm{E} \times_{\mathbb{Z}_{2}} \mathrm{SU}(2)\right)=M \mathrm{SO} \wedge \Sigma^{-3} M T \operatorname{Pin}^{+}(3)=M \mathrm{SO} \wedge \Sigma^{-3} M \operatorname{Spin}(3) \wedge \Sigma^{-2} M \mathbb{Z}_{2}, \\
M T\left(\operatorname{Pin}^{+} \times_{\mathbb{Z}_{2}} \mathrm{SU}(2)\right)=M \operatorname{Spin} \wedge \Sigma^{-3} M \mathrm{O}(3), \\
M T\left(\operatorname{Pin}^{-} \times_{\mathbb{Z}_{2}} \mathrm{SU}(2)\right)=M \operatorname{Spin} \wedge \Sigma^{3} M T \mathrm{O}(3) .
\end{array}\right.
$$

The $\mathrm{BSU}(2)_{+}$is the disjoint union of $\mathrm{BSU}(2)$ and a point, while $\Sigma$ is the suspension. From the short exact sequence $1 \rightarrow \mathrm{SO} \rightarrow \mathrm{E} \rightarrow \mathbb{Z}_{4} \rightarrow 1$, we have an induced short exact sequence

$$
1 \rightarrow \mathrm{SO} \rightarrow \mathrm{E} \times_{\mathbb{Z}_{2}} \mathrm{SU}(2) \rightarrow \mathrm{SU}(2) \times_{\mathbb{Z}_{2}} \mathbb{Z}_{4} \rightarrow 1
$$

Note that $\mathrm{SU}(2) \times_{\mathbb{Z}_{2}} \mathbb{Z}_{4}=\operatorname{Spin}(3) \times_{\mathbb{Z}_{2}} \mathbb{Z}_{4}=\operatorname{Pin}^{+}(3)$, so $M T\left(\mathrm{E} \times \mathbb{Z}_{2} \mathrm{SU}(2)\right)=M \mathrm{SO} \wedge \Sigma^{-3} M T \mathrm{Pin}^{+}(3)=M \mathrm{SO} \wedge$ $\Sigma^{-3} M \operatorname{Spin}(3) \wedge \Sigma^{-2} M \mathbb{Z}_{2}$.
Let $M$ be an $n$ manifold, and $V_{\mathrm{SO}(3)}$ be the associated vector bundle of the $\mathrm{SO}(3)$ gauge bundle. Below we compute the Stiefel-Whitney classes of $(T M-n) \otimes V_{\mathrm{SO}(3)}$. They are used to express the cobordism invariants of $\Omega_{d}^{\operatorname{Pin}^{ \pm} \times_{\mathbb{Z}_{2}} \mathrm{SU}(2)}$. Below $w_{i}$ means the $i$ th Stiefel-Whitney class, and $w$ means the total Stiefel-Whitney class; namely, we have $w=1+w_{1}+w_{2}+w_{3}+\cdots$. We denote $w_{i}^{\prime}=$ $w_{i}\left(V_{\mathrm{SO}(3)}\right)$ and $\tilde{w}_{i}=w_{i}\left((T M-n) \otimes V_{\mathrm{SO}(3)}\right)$. In addition, the $w_{i}(T M)$ means specifically the $i$ th Stiefel-Whitney class of spacetime tangent bundle $T M$ :

$$
\begin{aligned}
w\left((T M-n) \otimes V_{\mathrm{SO}(3)}\right) & =\frac{w\left(T M \otimes V_{\mathrm{SO}(3)}\right)}{w\left(V_{\mathrm{SO}(3)}\right)^{n}} \\
& =\frac{1+w_{1}(T M)+w_{1}(T M)^{2}+w_{2}(T M)+n w_{2}^{\prime}+w_{1}(T M)^{3}+n w_{1}(T M) w_{2}^{\prime}+w_{3}(T M)+n w_{3}^{\prime}+\cdots}{\left(1+w_{2}^{\prime}+w_{3}^{\prime}+\cdots\right)^{n}} \\
& =1+w_{1}(T M)+w_{1}(T M)^{2}+w_{2}(T M)+w_{1}(T M)^{3}+w_{3}(T M)+\cdots
\end{aligned}
$$

So $\quad \tilde{w}_{1}=w_{1}(T M), \quad \tilde{w}_{2}=w_{1}(T M)^{2}+w_{2}(T M), \quad \tilde{w}_{3}=$ $w_{1}(T M)^{3}+w_{3}(T M)$, etc.,

We also use the notation TP for the classification of topological phases defined in [41], such that

$$
\operatorname{TP}_{d, \text { tor }}\left(G^{\prime}\right)=\Omega_{d, \text { tor }}^{G^{\prime}}
$$

Here are the list of tables summarizing the results in $4 \mathrm{~d}$ and in 5d: Tables III, IV, V, and VI.

We conclude this section with a summary. The four siblings of $4 \mathrm{~d} \mathrm{SU}(2)_{\theta=\pi} \mathrm{YM}$ theories are obtained, specifically, from summing over the $\mathrm{SU}(2)$ gauge connections of the following four topological terms (i.e., gauging the $\mathrm{SU}(2)$ global symmetry of the following four distinct SPTs):

(1) $(-1)^{c_{2}}$ in Eq. (3.5).

(2) $(-1)^{c_{2}}$ in Eq. (3.7). (See footnote 32.)

(3) $\exp \left(2 \pi \mathrm{i} \nu \eta_{\mathrm{SU}(2)}\right)$ with an odd class of $\nu=1,3 \in \mathbb{Z}_{4}$ in Eq. (3.10).

(4) $(-1)^{N_{0}^{\prime}}$ in Eq. (3.12).

These four theories exactly map to the enumeration of four gauge theories in Sec. III A. Adding other SPTs/bordism invariants such as $(-1)^{w_{1}(T M)^{4}}$ and $(-1)^{w_{2}(T M)^{2}}$ (and then dynamically gauging them), do not alter or gain new classes of gauge theories. The only tensor product is the gauge theory with $4 \mathrm{~d}$ SPTs, namely $\left(4 \mathrm{~d} \mathrm{SU}(2)_{\theta=\pi} \mathrm{YM}\right) \otimes(4 \mathrm{~d}$ SPTs). ${ }^{34}$

\section{TIME-REVERSAL SYMMETRY-ENRICHED 5D HIGHER-GAUGE TQFTS}

\section{A. Partition function of $5 \mathrm{~d}$ higher-gauge TQFTs}

Following the discussions of four classes of $5 \mathrm{~d}$ timereversal and one-form center symmetry $\mathbb{Z}_{2}^{T} \times \mathbb{Z}_{2,[1]}^{e}$ higherSPTs $\mathbf{Z}_{\mathrm{SPT}_{\left(K_{1}, K_{2}\right)}^{5 \mathrm{~d}}}^{\mathrm{d}}\left[M^{5}\right]$ in Sec. IIF 1 with their partition functions in Eq. (2.61), we proceed to dynamically gauge the one-form symmetry $\mathbb{Z}_{2,[1]}^{e}$. Then we obtain the $5 \mathrm{~d}$ timereversal symmetric enriched topologically ordered state (SETs) with two-form $\mathbb{Z}_{2}$-valued dynamical $B$ gauge fields. We expect a precise mathematical formulation requires a certain version of higher category theory. Below we instead

\footnotetext{
${ }^{34}$ For the classification of the gauge theory, we identify the following phases:

$$
\text { (gauge theory) } \otimes(\text { SPTs }) \simeq \text { (gauge theory }) \text {. }
$$

For the classification of $4 \mathrm{~d} \mathrm{SU}(2)_{\theta=\pi} \mathrm{YM}$, we identify the following phases:

$$
\left(4 \mathrm{~d} \mathrm{SU}(2)_{\theta=\pi} \mathrm{YM}\right) \otimes(4 \mathrm{~d} \mathrm{SPTs}) \simeq\left(4 \mathrm{~d} \mathrm{SU}(2)_{\theta=\pi} \mathrm{YM}\right)
$$

See more physically motivated discussions in [38] and References therein.
} 
TABLE VII. Partition function $\mathbf{Z}\left(M^{5}\right)$ and topological degeneracy of $5 \mathrm{~d}$ higher-SPTs, for example, $\mathbf{Z}_{\mathrm{SPT}_{B S q^{1} B}}\left(M^{5}\right):=(-1)^{\int_{M^{5}} B \mathrm{Sq}^{1} B}$. The notations $\alpha, \beta, \gamma, \zeta$ are explained in the computation below in Sec. IV B 3.

\begin{tabular}{lcccc}
\hline \hline $\mathbf{Z}\left(M^{5}\right)$ with $M^{5}:$ & $(\mathrm{W}, 0)$ & $\left(S^{1} \times \mathbb{R} \mathbb{P}^{2} \times \mathbb{R} \mathbb{P}^{2}, \gamma \alpha_{1}\right)$ & $\left(S^{1} \times \mathbb{R P}^{4}, \gamma \zeta\right)$ & $\left(\mathbb{R} \mathbb{P}^{2} \times \mathbb{R} \mathbb{P}^{3}, \alpha \beta\right)$ \\
\hline $\mathbf{Z}_{\mathrm{SPT}}^{\mathrm{trivial}}\left(M^{5}\right)$ & 1 & 1 & 1 & 1 \\
$\mathbf{Z}_{\mathrm{SPT}_{B S q^{1} B}}\left(M^{5}\right)$ & 1 & 1 & 1 & -1 \\
$\mathbf{Z}_{\mathrm{SPT}_{\mathrm{Sq}^{2} \mathrm{Sq}^{1} B}}\left(M^{5}\right)$ & 1 & 1 & -1 & 1 \\
$\mathbf{Z}_{\mathrm{SPT}_{w_{1}(T M)^{2} \mathrm{Sq}^{1} B}\left(M^{5}\right)}$ & 1 & -1 & -1 & 1 \\
$\mathbf{Z}_{\mathrm{SPT}_{w_{2}(T M) \mathrm{Sq}^{1} B}\left(M^{5}\right)}$ & 1 & -1 & 1 & 1 \\
\hline \hline
\end{tabular}

approach from a higher-gauge TQFT perspective. We can define the four classes of $5 \mathrm{~d}$ partition functions $\mathbf{Z}_{\mathrm{SET}_{\left(K_{1}, K_{2}\right)}}^{5 \mathrm{~d}}\left[M^{5}\right]$ as

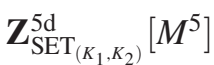

$\equiv \frac{\left|H^{0}\left(M, \mathbb{Z}_{2}\right)\right|}{\left|H^{1}\left(M, \mathbb{Z}_{2}\right)\right|} \sum_{B \in H^{2}\left(M^{5}, \mathbb{Z}_{2}\right)} \mathrm{e}^{\mathrm{i} \pi \int_{M^{5}} \frac{1}{2} \tilde{w}_{1}(T M) \cup \mathcal{P}(B)+K_{1} w_{1}(T M)^{2} \mathrm{Sq}^{1} B}$

$$
\begin{aligned}
= & \frac{\left|H^{0}\left(M, \mathbb{Z}_{2}\right)\right|}{\left|H^{1}\left(M, \mathbb{Z}_{2}\right)\right|} \\
& \times \sum_{B \in H^{2}\left(M^{5}, \mathbb{Z}_{2}\right)} \mathrm{e}^{\mathrm{i} \pi \int_{M^{5}} B \mathrm{Sq}^{1} B+\left(1+K_{1}\right) w_{1}(T M)^{2} \mathrm{Sq}^{1} B+w_{2}(T M) \mathrm{Sq}^{1} B}
\end{aligned}
$$$$
=\frac{\left|H^{0}\left(M, \mathbb{Z}_{2}\right)\right|}{\left|H^{1}\left(M, \mathbb{Z}_{2}\right)\right|} \sum_{\substack{B, b, h \in \mathrm{C}^{2}\left(M^{5}, \mathbb{Z}_{2}\right) \\ c \in \mathrm{C}^{3}\left(M^{5}, \mathbb{Z}_{2}\right)}} \exp \left(\mathrm{i} \pi \int_{M^{5}} \delta w_{1}(T M) \cup c\right.
$$$$
+\delta w_{2}(T M) \cup h+b \cup \delta B+B \mathrm{Sq}^{1} B
$$$$
\left.+\left(1+K_{1}\right) w_{1}(T M)^{2} \mathrm{Sq}^{1} B+w_{2}(T M) \mathrm{Sq}^{1} B\right)
$$$$
\cong \int[\mathcal{D} B][\mathcal{D} b][\mathcal{D} h][\mathcal{D} c] \exp \left(\mathrm{i} \pi \int_{M^{5}}\left(\mathrm{~d} w_{1}(T M)\right) c\right.
$$$$
+\left(\mathrm{d} w_{2}(T M)\right) h+b \mathrm{~d} B+B \frac{1}{2} \mathrm{~d} B
$$$$
\left.+\left(1+K_{1}\right) w_{1}(T M)^{2} \frac{1}{2} \mathrm{~d} B+w_{2}(T M) \frac{1}{2} \mathrm{~d} B\right) .
$$

In the last step (under the symbol $\cong$ ), we have converted the 5d higher-cochain TQFT to 5d higher-form gauge field continuum TQFT for $\mathbf{Z}_{\mathrm{SET}_{\left(K_{1}, K_{2}\right)}}^{5 \mathrm{~d}}\left[M^{5}\right]$. Moreover, we can insert extended operators (say $U, X, Y, \ldots$ ) into the path integral:

$$
\begin{aligned}
& \mathbf{Z}_{\mathrm{SET}_{\left(K_{1}, K_{2}\right)}}^{5 \mathrm{~d}}\left[M^{5} ; U, X, Y, \ldots\right] \\
& \equiv \int[\mathcal{D} B][\mathcal{D} b][\mathcal{D} h][\mathcal{D} c] U \cdot X \cdot Y \cdots \\
& \quad \times \exp \left(\mathrm{i} \pi \int_{M^{5}}\left(\mathrm{~d} w_{1}(T M)\right) c+\left(\mathrm{d} w_{2}(T M)\right) h+b \mathrm{~d} B\right. \\
& \left.\quad+B \frac{1}{2} \mathrm{~d} B+\left(1+K_{1}\right) w_{1}(T M)^{2} \frac{1}{2} \mathrm{~d} B+w_{2}(T M) \frac{1}{2} \mathrm{~d} B\right) .
\end{aligned}
$$

Note that since $K_{2} \mathrm{Sq}^{1}\left(w_{2}(T M) B\right)$ is trivial for closed five manifolds, the partition function $\mathbf{Z}_{\mathrm{SET}_{\left(K_{1}, K_{2}\right)}}^{5 \mathrm{~d}}\left[M^{5}\right]$ and the correlation function computed from the path integral

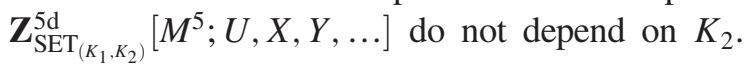

\section{B. Partition function and topological degeneracy}

Below we compute the partition function $\mathbf{Z}\left(M^{5}\right)$ on closed manifolds $M^{5}$. When $M^{5}=M^{4} \times S^{1}$, we can interpret it as topological GSD of TQFT. Our computations follow the strategy in $[13,15]$. We directly summarize the results in Tables VII, VIII, and IX.

TABLE VIII. Partition function $\mathbf{Z}\left(M^{5}\right)$ and topological degeneracy of $5 \mathrm{~d}$ higher-SETs, $\mathbf{Z}_{\mathrm{SET}_{\left(K_{1}, K_{2}\right)}}\left(M^{5}\right):=$ $\frac{\left|H^{0}\left(M^{5}, \mathbb{Z}_{2}\right)\right|}{\left|H^{1}\left(M^{5}, \mathbb{Z}_{2}\right)\right|} \sum_{B \in H^{2}\left(M^{5}, \mathbb{Z}_{2}\right)}(-1) \int_{M^{5}} B \mathrm{Sq}^{1} B+\left(1+K_{1}\right) w_{1}(T M)^{2} \mathrm{Sq}^{1} B+w_{2}(T M) \mathrm{Sq}^{1} B$

\begin{tabular}{lccccccccc}
\hline $\mathbf{Z}\left(M^{5}\right)$ with $M^{5}:$ & $T^{5}$ & $S^{1} \times S^{4}$ & $S^{1} \times \mathbb{R P}$ & $T^{2} \times S^{3}$ & $S^{1} \times S^{2} \times S^{2}$ & $S^{1} \times \mathbb{R} \mathbb{P}^{2} \times \mathbb{R} \mathbb{P}^{2}$ & $\mathbb{R P} \mathbb{P}^{2} \times \mathbb{R} \mathbb{P}^{3}$ & $S^{5}$ & $\mathrm{~W}$ \\
\hline $\mathbf{Z}_{2 \text {-form } B}^{\text {untwist }}\left(M^{5}\right)$ & $\frac{2^{10} \cdot 2}{2^{5}}=64$ & $\frac{2^{0} \cdot 2}{2^{1}}=1$ & $\frac{2^{2} \cdot 2}{2^{2}}=2$ & $\frac{2^{1} \cdot 2}{2^{2}}=1$ & $\frac{2^{2} \cdot 2}{2^{1}}=4$ & $\frac{2^{5} \cdot 2}{2^{3}}=8$ & $\frac{2^{3} \cdot 2}{2^{2}}=4$ & $\frac{2^{0} \cdot 2}{2^{0}}=1$ & 4 \\
$\mathbf{Z}_{\mathrm{SET}_{(0,0)}}\left(M^{5}\right)$ & 64 & 1 & 1 & 1 & 4 & 2 & 2 & 2 \\
$\mathbf{Z}_{\mathrm{SET}_{(1,0)}}\left(M^{5}\right)$ & 64 & 1 & 1 & 1 & 4 & 2 & 2 & 2 \\
$\mathbf{Z}_{\mathrm{SET}_{(0,1)}}\left(M^{5}\right)$ & 64 & 1 & 1 & 1 & 4 & 2 & 2 & 2 \\
$\mathbf{Z}_{\mathrm{SET}_{(1,1)}}\left(M^{5}\right)$ & 64 & 1 & 1 & 1 & 4 & 2 & 2 & 2 & 4 \\
\hline \hline
\end{tabular}


TABLE IX. Partition function $\mathbf{Z}\left(M^{5}\right)$ and topological degeneracy of $5 \mathrm{~d}$ higher SETs, for example, $\mathbf{Z}_{\mathrm{SET}_{B S q^{1} B}}\left(M^{5}\right):=$ $\frac{\left|H^{0}\left(M^{5}, \mathbb{Z}_{2}\right)\right|}{\left|H^{1}\left(M^{5}, \mathbb{Z}_{2}\right)\right|} \sum_{B \in H^{2}\left(M^{5}, \mathbb{Z}_{2}\right)}(-1)^{\int_{M^{5}} B S q^{1} B}$.

\begin{tabular}{|c|c|c|c|c|}
\hline $\mathbf{Z}\left(M^{5}\right)$ with $M^{5}$ : & $\mathrm{W}$ & $S^{1} \times \mathbb{R} \mathbb{P}^{2} \times \mathbb{R} \mathbb{P}^{2}$ & $S^{1} \times \mathbb{R P}^{4}$ & $\mathbb{R} \mathbb{P}^{2} \times \mathbb{R} \mathbb{P}^{3}$ \\
\hline $\mathbf{Z}_{2 \text {-form } B}^{\text {untwist }}\left(M^{5}\right)$ & 4 & 8 & 2 & 4 \\
\hline $\mathbf{Z}_{\mathrm{SET}_{B S q^{1} B} D}\left(M^{5}\right)$ & 0 & 2 & 1 & 2 \\
\hline $\mathbf{Z}_{\mathrm{SET}_{\mathrm{Sq}^{2} \mathrm{~S}_{1} B}}\left(M^{5}\right)$ & 0 & 8 & 0 & 4 \\
\hline $\mathbf{Z}_{\mathrm{SET}_{w_{1}(T M)^{2} \mathrm{Sq}^{1} B}}\left(M^{5}\right)$ & 4 & 0 & 0 & 4 \\
\hline $\mathbf{Z}_{\mathrm{SET}_{w_{2}(T M) \mathrm{Sq}^{1} B}}\left(M^{5}\right)$ & 0 & 0 & 2 & 4 \\
\hline
\end{tabular}

\section{5d SPTs as short-range entangled invertible TQFTs}

We evaluate the partition function of various $5 \mathrm{~d}$ iTQFTs on various manifolds and enumerate the results in Table VII. Below we denote the five-dimensional $\mathrm{Wu}$ manifold as $\mathrm{W} \equiv \mathrm{SU}(3) / \mathrm{SO}(3)$.

\section{5d SETs, as long-range entangled TQFTs}

We evaluate the partition function of various $5 \mathrm{~d}$ TQFTs (as 5d SETs) on various manifolds, and enumerate the results in Tables VIII and IX.

\section{Computation}

Now we illustrate our computation ${ }^{35}$ :

(1) For $M=S^{1} \times \mathbb{R P}^{4}$, let $\gamma$ be the generator of $H^{1}\left(S^{1}, \mathbb{Z}_{2}\right)=\mathbb{Z}_{2}$ and $\zeta$ be the generator of $H^{1}\left(\mathbb{R P}^{4}, \mathbb{Z}_{2}\right)=\mathbb{Z}_{2}$. Note that $w_{1}(T M)=\zeta$. The cohomology groups have that $H^{0}\left(S^{1} \times \mathbb{R P}^{4}\right.$, $\left.\mathbb{Z}_{2}\right)=\mathbb{Z}_{2}, H^{1}\left(S^{1} \times \mathbb{R P}^{4}, \mathbb{Z}_{2}\right)=\mathbb{Z}_{2}^{2}$, and $H^{2}\left(S^{1} \times\right.$ $\left.\mathbb{R} P^{4}, \mathbb{Z}_{2}\right)=\mathbb{Z}_{2}^{2}$ whose two generators are $\gamma \zeta$ and $\zeta^{2}$. If $B=\lambda_{1} \gamma \zeta+\lambda_{2} \zeta^{2}$, then $\operatorname{Sq}^{1} B=\lambda_{1} \gamma \zeta^{2}$. Hence

$$
\begin{gathered}
\int_{S^{1} \times \mathbb{R} \mathbb{P}^{4}} B \mathrm{Sq}^{1} B=\lambda_{1} \lambda_{2}, \\
\int_{S^{1} \times \mathbb{R P}^{4}} B \mathrm{Sq}^{1} B+w_{1}(T M)^{2} \mathrm{Sq}^{1} B=\lambda_{1} \lambda_{2}+\lambda_{1} .
\end{gathered}
$$

On the other hand, since $w_{2}(T M)=0$ for $S^{1} \times \mathbb{R} \mathbb{P}^{4}$, we have

$$
\begin{aligned}
\mathbf{Z}_{\mathrm{SET}_{(0,0)}}\left(S^{1} \times \mathbb{R} \mathbb{P}^{4}\right) & =\mathbf{Z}_{\mathrm{SET}_{(0,1)}}\left(S^{1} \times \mathbb{R} \mathbb{P}^{4}\right) \\
& =\frac{1}{2} \sum_{\lambda_{1}, \lambda_{2} \in \mathbb{Z}_{2}}(-1)^{\lambda_{1}\left(\lambda_{2}+1\right)},
\end{aligned}
$$

\footnotetext{
${ }^{35}$ Interestingly, we notice that the $\mathrm{Wu}$ manifold $\mathrm{W}$ can assign a closely related but different partition function $\mathbf{Z}_{\mathrm{SET}}^{\prime}(\mathrm{W})$ with a distinct value: $\mathbf{Z}_{\mathrm{SET}}^{\prime}(\mathrm{W}):=\frac{\left|H^{0}\left(M^{5}, \mathbb{Z}_{2}\right)\right|}{\left|H^{1}\left(M^{5}, \mathbb{Z}_{2}\right)\right|} \times$ $\sum_{B \in H^{2}\left(M^{5}, \mathbb{Z}_{2}\right)}(-1) \int_{M^{5}} B \mathrm{Sq}^{1} B+\left(1+K_{1}\right) w_{1}(T M)^{2} \mathrm{Sq}^{1} B=0$.
}

$$
\begin{aligned}
\mathbf{Z}_{\mathrm{SET}_{(1,0)}}\left(S^{1} \times \mathbb{R} \mathbb{P}^{4}\right) & =\mathbf{Z}_{\operatorname{SET}_{(1,1)}}\left(S^{1} \times \mathbb{R P}^{4}\right) \\
& =\frac{1}{2} \sum_{\lambda_{1}, \lambda_{2} \in \mathbb{Z}_{2}}(-1)^{\lambda_{1} \lambda_{2}} .
\end{aligned}
$$

Since the number of $\left(\lambda_{1}, \lambda_{2}\right)$ satisfying the constraint $\lambda_{1} \lambda_{2}=1$ is only one,

$$
\#\left\{\left(\lambda_{1}, \lambda_{2}\right) \in \mathbb{Z}_{2}^{2} \mid \lambda_{1} \lambda_{2}=1\right\}=1
$$

also note that changing $\lambda_{2}$ to $\lambda_{2}+1$ does not affect the sum, so

$$
\begin{aligned}
\mathbf{Z}_{\mathrm{SET}_{(0,0)}}\left(S^{1} \times \mathbb{R} \mathbb{P}^{4}\right) & =\mathbf{Z}_{\mathrm{SET}_{(1,0)}}\left(S^{1} \times \mathbb{R} \mathbb{P}^{4}\right) \\
& =\mathbf{Z}_{\mathrm{SET}_{(0,1)}}\left(S^{1} \times \mathbb{R} \mathbb{P}^{4}\right) \\
& =\mathbf{Z}_{\mathrm{SET}_{(1,1)}}\left(S^{1} \times \mathbb{R} \mathbb{P}^{4}\right) \\
& =\frac{1}{2}(3-1)=1 .
\end{aligned}
$$

(2) For $M=\mathbb{R} \mathbb{P}^{2} \times \mathbb{R} \mathbb{P}^{3}$, let $\alpha$ be the generator of $H^{1}\left(\mathbb{R P}^{2}, \mathbb{Z}_{2}\right)=\mathbb{Z}_{2}$ and $\beta$ be the generator of $H^{1}\left(\mathbb{R P}^{3}, \mathbb{Z}_{2}\right)=\mathbb{Z}_{2}$. Note that $w_{1}(T M)=\alpha$. $H^{0}\left(\mathbb{R} \mathbb{P}^{2} \times \mathbb{R} \mathbb{P}^{3}, \mathbb{Z}_{2}\right)=\mathbb{Z}_{2}, H^{1}\left(\mathbb{R P}^{2} \times \mathbb{R} \mathbb{P}^{3}, \mathbb{Z}_{2}\right)=$ $\mathbb{Z}_{2}^{2}, H^{2}\left(\mathbb{R} \mathbb{P}^{2} \times \mathbb{R} \mathbb{P}^{3}, \mathbb{Z}_{2}\right)=\mathbb{Z}_{2}^{3}$ whose three generators are $\alpha^{2}, \beta^{2}$, and $\alpha \beta$. If $B=\lambda_{1} \alpha^{2}+\lambda_{2} \beta^{2}+\lambda_{3} \alpha \beta$, then $\mathrm{Sq}^{1} B=\lambda_{3} \alpha^{2} \beta+\lambda_{3} \alpha \beta^{2}$. Hence

$$
\begin{gathered}
\int_{\mathbb{R P}^{2} \times \mathbb{R}^{3}} B \mathrm{Sq}^{1} B=\lambda_{3}^{2}+\lambda_{2} \lambda_{3}, \\
\int_{\mathbb{R}^{2} \times \mathbb{R P}^{3}} B \operatorname{Sq}^{1} B+w_{1}(T M)^{2} \mathrm{Sq}^{1} B=\lambda_{3}^{2}+\lambda_{2} \lambda_{3} .
\end{gathered}
$$

On the other hand, since $w_{2}(T M)+w_{1}(T M)^{2}=0$ for $\mathbb{R} \mathbb{P}^{2} \times \mathbb{R} \mathbb{P}^{3}$, so

$$
\begin{aligned}
& \mathbf{Z}_{\mathrm{SET}_{(0,0)}}\left(\mathbb{R} \mathbb{P}^{2} \times \mathbb{R} \mathbb{P}^{3}\right)=\frac{1}{2} \sum_{\lambda_{1}, \lambda_{2}, \lambda_{3} \in \mathbb{Z}_{2}}(-1)^{\lambda_{3}^{2}+\lambda_{2} \lambda_{3}}, \\
& \mathbf{Z}_{\mathrm{SET}_{(1,0)}}\left(\mathbb{R P} \mathbb{P}^{2} \times \mathbb{R} \mathbb{P}^{3}\right)=\frac{1}{2} \sum_{\lambda_{1}, \lambda_{2}, \lambda_{3} \in \mathbb{Z}_{2}}(-1)^{\lambda_{3}^{2}+\lambda_{2} \lambda_{3}}
\end{aligned}
$$

Since

$\#\left\{\left(\lambda_{1}, \lambda_{2}, \lambda_{3}\right) \in \mathbb{Z}_{2}^{3} \mid \lambda_{3}^{2}+\lambda_{2} \lambda_{3}=1\right\}=2$, 
so

$$
\begin{aligned}
\mathbf{Z}_{\mathrm{SET}_{(0,0)}}\left(\mathbb{R} \mathbb{P}^{2} \times \mathbb{R} \mathbb{P}^{3}\right) & =\mathbf{Z}_{\mathrm{SET}_{(1,0)}}\left(\mathbb{R} \mathbb{P}^{2} \times \mathbb{R} \mathbb{P}^{3}\right) \\
& =\mathbf{Z}_{\mathrm{SET}_{(0,1)}}\left(\mathbb{R} \mathbb{P}^{2} \times \mathbb{R} \mathbb{P}^{3}\right) \\
& =\mathbf{Z}_{\mathrm{SET}_{(1,1)}}\left(\mathbb{R} \mathbb{P}^{2} \times \mathbb{R} \mathbb{P}^{3}\right) \\
& =\frac{1}{2}(6-2)=2 .
\end{aligned}
$$

(3) For $M=S^{1} \times \mathbb{R} \mathbb{P}^{2} \times \mathbb{R} \mathbb{P}^{2}$, let $\gamma$ be the generator of $H^{1}\left(S^{1}, \mathbb{Z}_{2}\right)=\mathbb{Z}_{2}$ and $\alpha_{i}$ be the generator of $H^{1}\left(\mathbb{R} \mathbb{P}^{2}, \mathbb{Z}_{2}\right)=\mathbb{Z}_{2}$ of the $i$ th factor $\mathbb{R} \mathbb{P}^{2}(i=1,2)$. Note that $w_{1}(T M)=\alpha_{1}+\alpha_{2} . \quad H^{0}\left(S^{1} \times \mathbb{R}^{2} \times\right.$ $\left.\mathbb{R P}^{2}, \mathbb{Z}_{2}\right)=\mathbb{Z}_{2}, \quad H^{1}\left(S^{1} \times \mathbb{R} \mathbb{P}^{2} \times \mathbb{R} \mathbb{P}^{2}, \mathbb{Z}_{2}\right)=\mathbb{Z}_{2}^{3}$, $H^{2}\left(S^{1} \times \mathbb{R} \mathbb{P}^{2} \times \mathbb{R} \mathbb{P}^{2}, \mathbb{Z}_{2}\right)=\mathbb{Z}_{2}^{5}$ whose five generators are $\alpha_{1}^{2}, \alpha_{2}^{2}, \gamma \alpha_{1}, \gamma \alpha_{2}$, and $\alpha_{1} \alpha_{2}$. If $B=$ $\lambda_{1} \alpha_{1}^{2}+\lambda_{2} \alpha_{2}^{2}+\lambda_{3} \gamma \alpha_{1}+\lambda_{4} \gamma \alpha_{2}+\lambda_{5} \alpha_{1} \alpha_{2}$, then $\mathrm{Sq}^{1} B=$ $\lambda_{3} \gamma \alpha_{1}^{2}+\lambda_{4} \gamma \alpha_{2}^{2}+\lambda_{5} \alpha_{1}^{2} \alpha_{2}+\lambda_{5} \alpha_{1} \alpha_{2}^{2}$. Hence

$\int_{S^{1} \times \mathbb{R}^{2} \times \mathbb{R}^{2}} B \mathrm{Sq}^{1} B=\lambda_{1} \lambda_{4}+\lambda_{2} \lambda_{3}+\lambda_{3} \lambda_{5}+\lambda_{4} \lambda_{5}$,

$$
\begin{aligned}
& \int_{S^{1} \times \mathbb{R}^{2} \times \mathbb{R} \mathbb{P}^{2}} B \mathrm{Sq}^{1} B+w_{1}(T M)^{2} \mathrm{Sq}^{1} B \\
& =\lambda_{1} \lambda_{4}+\lambda_{2} \lambda_{3}+\lambda_{3} \lambda_{5}+\lambda_{4} \lambda_{5}+\lambda_{3}+\lambda_{4} .
\end{aligned}
$$

On the other hand, since $w_{2}(T M)+w_{1}(T M)^{2}=0$ for $S^{1} \times \mathbb{R} \mathbb{P}^{2} \times \mathbb{R} \mathbb{P}^{2}$, so

$$
\begin{aligned}
& \mathbf{Z}_{\mathrm{SET}_{(0,0)}}\left(S^{1} \times \mathbb{R} \mathbb{P}^{2} \times \mathbb{R} \mathbb{P}^{2}\right) \\
&= \frac{1}{4} \sum_{\lambda_{1}, \lambda_{2}, \lambda_{3}, \lambda_{4}, \lambda_{5} \in \mathbb{Z}_{2}}(-1)^{\lambda_{1} \lambda_{4}+\lambda_{2} \lambda_{3}+\lambda_{3} \lambda_{5}+\lambda_{4} \lambda_{5}}, \\
& \mathbf{Z}_{\mathrm{SET}_{(1,0)}}\left(S^{1} \times \mathbb{R} \mathbb{P}^{2} \times \mathbb{R} \mathbb{P}^{2}\right) \\
&=\frac{1}{4} \sum_{\lambda_{1}, \lambda_{2}, \lambda_{3}, \lambda_{4}, \lambda_{5} \in \mathbb{Z}_{2}}(-1)^{\lambda_{1} \lambda_{4}+\lambda_{2} \lambda_{3}+\lambda_{3}\left(\lambda_{5}+1\right)+\lambda_{4}\left(\lambda_{5}+1\right) .}
\end{aligned}
$$

Since

$$
\begin{gathered}
\#\left\{\left(\lambda_{1}, \lambda_{2}, \lambda_{3}, \lambda_{4}, \lambda_{5}\right) \in \mathbb{Z}_{2}^{5} \mid \lambda_{1} \lambda_{4}+\lambda_{2} \lambda_{3}+\lambda_{3} \lambda_{5}+\lambda_{4} \lambda_{5}\right. \\
\quad=1\}=12,
\end{gathered}
$$

also note that changing $\lambda_{5}$ to $\lambda_{5}+1$ does not affect the sum, so

$$
\begin{gathered}
\mathbf{Z}_{\operatorname{SET}_{(0,0)}}\left(S^{1} \times \mathbb{R} \mathbb{P}^{2} \times \mathbb{R} \mathbb{P}^{2}\right)=\mathbf{Z}_{\mathrm{SET}_{(1,0)}}\left(S^{1} \times \mathbb{R} \mathbb{P}^{2} \times \mathbb{R} \mathbb{P}^{2}\right) \\
=\mathbf{Z}_{\mathrm{SET}_{(0,1)}}\left(S^{1} \times \mathbb{R} \mathbb{P}^{2} \times \mathbb{R} \mathbb{P}^{2}\right)=\mathbf{Z}_{\operatorname{SET}_{(1,1)}\left(S^{1} \times \mathbb{R} \mathbb{P}^{2} \times \mathbb{R} \mathbb{P}^{2}\right)=\frac{1}{4}(20-12)=2 .}
\end{gathered}
$$

(4) For a $5 \mathrm{~d} \mathrm{Wu}$ manifold $\mathrm{W}=\mathrm{SU}(3) / \mathrm{SO}(3)$, with $H^{0}\left(\mathrm{~W}, \mathbb{Z}_{2}\right)=\mathbb{Z}_{2}, H^{1}\left(\mathrm{~W}, \mathbb{Z}_{2}\right)=0$, note that $w_{1}(T \mathrm{~W})=0, H^{2}\left(\mathrm{~W}, \mathbb{Z}_{2}\right)=\mathbb{Z}_{2}$ which is generated by $w_{2}(T \mathrm{~W}) . \mathrm{Sq}^{1} w_{2}(T \mathrm{~W})=w_{3}(T \mathrm{~W})$,

$\mathbf{Z}_{\mathrm{SET}_{(0,0)}}(\mathrm{W})=2 \sum_{B=0, w_{2}(T \mathrm{~W})}(-1)^{B \mathrm{Sq}^{1} B+w_{2}(T \mathrm{~W}) \mathrm{Sq}^{1} B}=4$,

so

$$
\begin{aligned}
\mathbf{Z}_{\mathrm{SET}_{(0,0)}}(\mathrm{W}) & =\mathbf{Z}_{\mathrm{SET}_{(1,0)}}(\mathrm{W}) \\
& =\mathbf{Z}_{\mathrm{SET}_{(0,1)}}(\mathrm{W})=\mathbf{Z}_{\mathrm{SET}_{(1,1)}}(\mathrm{W})=4 .
\end{aligned}
$$

In the next section, we will use the anyonic string/brane braiding statistics and the link invariants of 5d TQFTs to characterize and distinguish these $5 \mathrm{~d}$ SETs.

\section{ANYONIC STRING/BRANE BRAIDING STATISTICS AND LINK INVARIANTS OF 5D TQFTS}

Now we compute the path integral Eq. (4.5) with extended operator insertions. To recall the general definitions, we have

(i) Partition or path integral w/out insertion is

$$
\sum_{B \in \mathcal{C}^{2}\left(M, \mathbb{Z}_{2}\right)}\left(e^{\mathrm{i} S}\right)
$$

(ii) In physics, the vacuum expectation value (VEV) of a theory $S$ is defined as

$$
\langle\mathcal{O}\rangle_{(\mathrm{VEV})}=\frac{\langle\mathcal{O}\rangle_{(\mathrm{VEV})}}{\langle 1\rangle_{(\mathrm{VEV})}}=\frac{\sum_{B \in C^{2}\left(M, \mathbb{Z}_{2}\right)}\left(e^{\mathrm{i} S} \mathcal{O}\right)}{\sum_{B \in C^{2}\left(M, \mathbb{Z}_{2}\right)}\left(e^{\mathrm{i} S}\right)}=\frac{\sum_{B \in C^{2}\left(M, \mathbb{Z}_{2}\right)}\left(e^{\mathrm{i} S} \mathcal{O}\right)}{\mathbf{Z}}=\frac{\text { path integral with insertions } \mathcal{O}}{\text { path integral without insertions }} .
$$


For example, this includes the link invariant that we will focus on in this section:

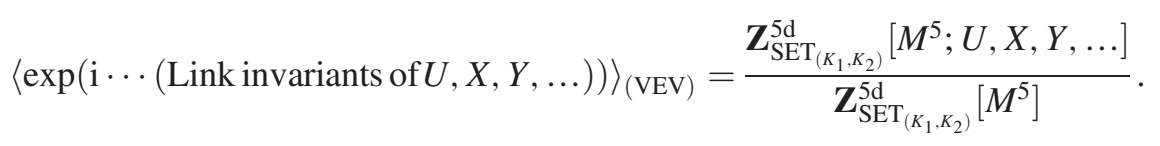

For conventions of our notations, we label the $1 \mathrm{~d}$ Wilson line as $W$, the $2 \mathrm{~d}$ surface operator as $U, U^{\prime}$, etc., We label the $3 \mathrm{~d}$ membrane operator as $X$ and the $4 d$ operator as $Y$, etc., We label the $d d$ hypersurface of general operators that we inserted as $\Sigma^{d}$, while we label this $\Sigma^{d}$ 's $(d+1) d$-Seiferthypervolume as $V^{d+1}$.

In this section, we focus on deriving the general link invariants for these $5 \mathrm{~d}$ TQFTs/SETs. ${ }^{36}$ In the next Sec. VI, we will provide explicit examples of the spacetime braiding process as the link configurations that can be detected by these link invariants derived here in Sec. V. The techniques for computing all these link invariants below are based on Ref. [12]. Below we simply apply the methods and notations introduced in Ref. [12].

Caveat: Note that while in the first section VA, we explicitly study the discrete cochain version of TQFT, in the sections below we implement the continuum formulation of TQFT. The reason is related to a fact that the graded noncommutativity of cochain fields is much more complicated to be dealt with than the continuum differential form fields. The subtle fact will be commented further in footnotes 37 and 38. We also note that when we deal with the continuum differential form fields later in Sec. V B to Sec. V D, we choose a normalization of differential form fields as $\oint B \in \mathbb{Z}$ with the periodicity $\oint B \sim \oint B+2$ (thus more similar to the convention of discrete cochain fields), instead of the more conventional $\oint B \in \pi \mathbb{Z}$ with the periodicity $\oint B \sim \oint B+2 \pi$.

\section{A. $\frac{1}{2} \tilde{w}_{1}(T M) \mathcal{P}(B)$ and a triple link invariant $\operatorname{Tlk}_{w_{1} B B}^{(5)}\left(\Sigma_{X}^{3}, \Sigma_{U_{(i)}}^{2}, \Sigma_{U_{(i i)}}^{2}\right)$}

We start with a $5 \mathrm{~d}$ TQFT obtained from summing over two-form field $B$ of $\frac{1}{2} \tilde{w}_{1}(T M) \mathcal{P}(B)$. This amounts to gauging the one-form $\mathbb{Z}_{2}$ of this $5 \mathrm{~d}$ SPTs. The resulting theory is $\mathbf{Z}_{\mathrm{SET}_{\left(K_{1}=0, K_{2}=0\right)}}$ in Eq. (4.5). The topological action and the partition function are

$$
\mathbf{S}=\pi \int_{M^{5}}\left(\frac{1}{2} \delta \tilde{w}_{1}(T M) \cup \tilde{c}+b \cup \delta B+\frac{1}{2} \tilde{w}_{1}(T M) \cup \mathcal{P}(B)\right),
$$

\footnotetext{
${ }^{36}$ For more guidance on the physical interpretations of link invariants, please see [12] and its introduction.
}

$$
\begin{gathered}
\mathbf{Z}=\int[\mathcal{D} B][\mathcal{D} \tilde{c}][\mathcal{D} b] \exp (\mathrm{i} \mathbf{S}) . \\
\mathbf{Z}=\sum_{\frac{B, b \in C^{2}\left(M^{5}, \mathbb{Z}_{2}\right)}{\tilde{\tilde{c}} \in C^{3}\left(M^{5}, \mathbb{Z}_{4}\right)}} \exp \left(\mathrm{i} \pi \int_{M^{5}} \frac{1}{2} \delta \tilde{w}_{1}(T M) \cup \tilde{c}+b \cup \delta B\right. \\
\left.+\frac{1}{2} \tilde{w}_{1}(T M) \cup \mathcal{P}(B)\right) .
\end{gathered}
$$

We consider the gauge transformation ${ }^{37}$ :

$$
\begin{aligned}
\tilde{w}_{1}(T M) & \rightarrow \tilde{w}_{1}(T M)+\delta \alpha, \\
B & \rightarrow B+\delta \beta, \\
\tilde{c} & \rightarrow \tilde{c}+\delta \gamma+\lambda, \\
b & \rightarrow b+\delta \zeta+\mu .
\end{aligned}
$$

under which the action transforms as

$$
\begin{aligned}
\mathbf{S} \rightarrow & \pi \int_{M^{5}} \frac{1}{2}\left(\tilde{w}_{1}(T M)+\delta \alpha\right)(B \cup B+B \cup \delta \beta+\delta \beta \cup B \\
& \left.+\delta \beta \cup \delta \beta+B \cup{ }_{1} \delta B+\delta \beta \cup \delta B\right) \\
& +\frac{1}{2} \delta \tilde{w}_{1}(T M)(\tilde{c}+\lambda)+(b+\mu) \delta B
\end{aligned}
$$

The gauge variance of the action is

$$
\begin{aligned}
\Delta \mathbf{S}= & \pi \int_{M^{5}} \frac{1}{2} \tilde{w}_{1}(T M)(\delta \beta \cup \delta \beta+2 \delta \beta \cup B+\delta(\delta \beta \cup B)) \\
& +\frac{1}{2} \delta \alpha(B \cup B+B \cup \underset{1}{1} \delta B+\delta \beta \cup \delta \beta \\
& +2 \delta \beta \cup B+\delta(\delta \beta \cup \underset{1}{ } \delta)) \\
& +\frac{1}{2} \delta \tilde{w}_{1}(T M) \lambda+\mu \delta B
\end{aligned}
$$

\footnotetext{
${ }^{37}$ One may consider adding additional terms on the gauge transformations, such as $\tilde{w}_{1}(T M) \rightarrow \tilde{w}_{1}(T M)+\delta \alpha(t, x)+\alpha_{1}(t, x)$ and $B \rightarrow B+\delta \beta(t, x)+\alpha_{2}(t, x)$, etc., However, terms such as $\alpha_{1}(t, x)=\alpha_{1}$ and $\alpha_{2}(t, x)=\alpha_{2}$ will need to be constant, which acts as the higher-form global symmetry transformation, instead of gauge transformation.
} 


$$
\begin{aligned}
= & \pi \int_{M^{5}} \frac{1}{2} \delta \tilde{w}_{1}(T M)(\beta \delta \beta)+\left(\delta \tilde{w}_{1}(T M)(\beta B)\right. \\
& \left.+\tilde{w}_{1}(T M) \beta \delta B\right)+\frac{1}{2} \delta \tilde{w}_{1}(T M)(\delta \beta \cup B) \\
& -\left(\alpha B \delta B+\frac{1}{2} \alpha u_{2} \delta B\right)-\alpha \delta \beta \delta B+\frac{1}{2} \delta \tilde{w}_{1}(T M) \lambda+\mu \delta B .
\end{aligned}
$$

In Eq. (5.8), we have used the formula ${ }^{38}$

$$
B \cup \delta \beta-\delta \beta \cup B+\delta \beta \cup \cup_{1} \delta B+\delta_{1}^{2} \beta \cup B=\delta(\delta \beta \cup B)
$$

and $\delta^{2} \beta=0$. In Eq. (5.9), we have used integration by part: for a closed five manifold without boundary, after integration by part we can drop the boundary term $\delta(\cdots)$. Since $\delta^{2} B=\delta^{2} \beta=\delta^{2} \alpha=0$, we drop $\delta \alpha\left(\delta \beta \cup \delta \beta+\delta\left(\delta \beta \cup_{1} B\right)\right)$ which has no effect on a closed five manifold without boundary. Denote $u_{2}=w_{2}(T M)+w_{1}(T M)^{2}$ as the second Wu class. We have also used the formula in footnote 37 as

$$
\begin{gathered}
B \cup \delta B-\delta B \cup B+\delta B \cup \delta B+B \cup{ }_{1} \delta^{2} B=\delta(B \cup \delta B), \\
\delta B \cup \delta B=\mathrm{Sq}^{2} \delta B=u_{2} \delta B .
\end{gathered}
$$

In Eq. (5.9), we used $\delta\left(\alpha\left(B \cup B+B \cup_{1} \delta B\right)\right)=\delta \alpha(B \cup B+$ $\left.B \cup_{1} \delta B\right)+\alpha\left(\delta B \cup B+B \cup \delta B+\delta\left(B \cup_{1} \delta B\right)\right)=\delta \alpha(B \cup B+$ $\left.B \cup_{1} \delta B\right)+\alpha\left(2 B \cup \delta B+u_{2} \delta B\right)$, and we dropped the total derivative term on a closed five manifold. The solution of gauge invariance, i.e., $\Delta \mathbf{S}=0$, imposes ${ }^{39}$

$$
\begin{aligned}
& \lambda=-\beta \delta \beta-2 \beta B-\delta \beta \cup \cup_{1} B \bmod 4 \\
& \mu=-\tilde{w}_{1}(T M) \beta+\alpha B+\frac{1}{2} \alpha u_{2}+\alpha \delta \beta \bmod 2 .
\end{aligned}
$$

The three-submanifold gauge invariant operator is

$$
\begin{aligned}
X & =\exp \left(\frac{\mathrm{i} \pi}{2} k\left(\int_{\Sigma^{3}} \tilde{c}+\int_{V^{4}} \mathcal{P}(B)\right)\right) \\
& =\exp \left(\frac{\mathrm{i} \pi}{2} k\left(\int_{M^{5}} \delta^{\perp}\left(\Sigma^{3}\right) \tilde{c}+\delta^{\perp}\left(V^{4}\right) \mathcal{P}(B)\right)\right),
\end{aligned}
$$

where $k \in \mathbb{Z}_{4}$. To verify the gauge invariance, we use $\mathcal{P}(B+\delta \beta)=\mathcal{P}(B)+\delta \beta \cup \delta \beta+2 \delta \beta \cup B+\delta\left(\delta \beta \cup_{1} B\right)$ and $\delta B=0$ on the four-submanifold Seifert volume $V^{4}$.

The two-submanifold (two-surface) operator gauge invariant is

$$
\begin{aligned}
U & =\exp \left(\mathrm{i} \pi \ell\left(\int_{\Sigma^{2}} b-\int_{V^{3}} \tilde{w}_{1}(T M) B-\frac{1}{2} \int_{V^{3}} \tilde{w}_{1}(T M) u_{2}\right)\right) \\
& =\exp \left(\mathrm{i} \pi \ell\left(\int_{M^{5}} b \delta^{\perp}\left(\Sigma^{2}\right)-\left(\tilde{w}_{1}(T M) B+\frac{1}{2} \tilde{w}_{1}(T M) u_{2}\right) \delta^{\perp}\left(V^{3}\right)\right)\right) \\
& =\exp \left(\mathrm{i} \pi \ell\left(\int_{M^{5}} b \delta^{\perp}\left(\Sigma^{2}\right)-\left(\tilde{w}_{1}(T M) B+\frac{1}{2} \tilde{w}_{1}(T M)\left(w_{2}(T M)+w_{1}(T M)^{2}\right)\right) \delta^{\perp}\left(V^{3}\right)\right)\right),
\end{aligned}
$$

${ }^{38}$ This is based on Steenrod's work "Products of Cocycles and Extensions of Mappings" [61], which derives

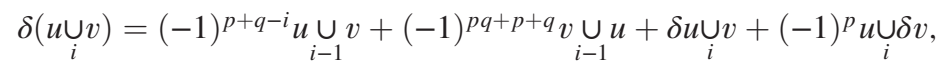

where $u \in C^{p}$ and $v \in C^{q}$.

${ }^{39}$ In general, when we study action Eq. (5.3), we have made a convenient choice with a term $\delta \tilde{w}_{1}(T M) \cup \tilde{c}$ instead of $\tilde{c} \cup \delta \tilde{w}_{1}(T M)$. For a generic three-cochain $x, \delta \tilde{w}_{1}(T M) x=x \delta \tilde{w}_{1}(T M)$ is not true; by Steenrod's formula in footnote $37 \mathrm{Eq} .(5.10), \delta \tilde{w}_{1}(T M) x=$ $x \delta \tilde{w}_{1}(T M)+\delta x \cup_{1} \delta \tilde{w}_{1}(T M)-\delta\left(x \cup_{1} \delta \tilde{w}_{1}(T M)\right)$, we can only drop the total derivative terms (i.e., the coboundary terms). In our present case, we consider $x=\frac{1}{2} \beta \delta \beta+\beta B+\frac{1}{2} \delta \beta \cup_{1} B$. So if $\delta x \cup_{1} \delta \tilde{w}_{1}(T M)$ is a coboundary, then we can also drop it, which results in

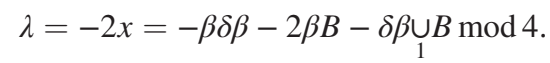

If $\delta x \cup_{1} \delta \tilde{w}_{1}(T M)$ is not a coboundary, we need the extra term

$$
\delta \tilde{w}_{1}(T M) x=x \delta \tilde{w}_{1}(T M)+\delta x \underset{1}{\cup} \delta \tilde{w}_{1}(T M)+\text { a total derivative/coboundary term. }
$$

When $\delta x \cup_{1} \delta \tilde{w}_{1}(T M)$ is not a coboundary, this results in a modified gauge transformation to $\lambda$. By writing the action as in Eq. (5.3), we can avoid additional complications, and thus we end up with a simpler gauge transformation Eq. (5.14). The graded noncommutativity of cochain fields is much more complicated than the case for continuum differential form fields. J.W. thanks Pierre Deligne for a discussion on the related issues. 
where $\ell \in \mathbb{Z}_{2}$ is an integer $\bmod 2$. To verify that $U$ is gauge invariant, we use $\delta B=\delta \tilde{w}_{1}(T M)=0$ on the three-submanifold Seifert volume $V^{3}$.

We insert $X, U_{(\mathbf{i})}$, and $U_{(\mathbf{i i})}$ into the path integral $\mathbf{Z}$, and write the correlation function either in the continuum field theory formulation or in the discrete cochain field theory formulation, interchangeably as

$$
\begin{aligned}
\left\langle X U_{(\mathbf{i})} U_{(\mathbf{i i})}\right\rangle & =\int[\mathcal{D} B][\mathcal{D} \tilde{c}][\mathcal{D} b] X U_{(\mathbf{i})} U_{(\mathbf{i i})} \exp (\mathbf{i S}), \\
\left\langle X U_{(\mathbf{i})} U_{(\mathbf{i i})}\right\rangle & =\sum_{\substack{B, b \in \mathcal{C}^{2}\left(M^{5}, \mathbb{Z}_{2}\right) \\
\tilde{c} \in \mathcal{C}^{3}\left(M^{5}, \mathbb{Z}_{4}\right)}} X U_{(\mathbf{i})} U_{(\mathbf{i i})} \exp \left(\mathrm{i} \pi \int_{M^{5}} \frac{1}{2} \delta \tilde{w}_{1}(T M) \cup \tilde{c}+b \cup \delta B+\frac{1}{2} \tilde{w}_{1}(T M) \cup \mathcal{P}(B)\right) .
\end{aligned}
$$

We compute the correlation functions as follows:

(1) Integrating out $\tilde{c}$ yields

$$
\begin{aligned}
\delta \tilde{w}_{1}(T M) & =k \delta^{\perp}\left(\Sigma_{X}^{3}\right), \\
\tilde{w}_{1}(T M) & =k \delta^{\perp}\left(V_{X}^{4}\right) ;
\end{aligned}
$$

hence as a consequence, $\delta^{2} \tilde{w}_{1}(T M)=\delta\left(k \delta^{\perp}\left(\Sigma_{W}^{3}\right)\right)=0$. So with the above configuration constraint, we get the double-counting mod 2 cancellation in the exponent of $\exp \left(\frac{\mathrm{i} \pi}{2} k\left(\int_{M^{5}} \delta^{\perp}\left(V_{X}^{4}\right) \mathcal{P}(B)\right)\right) \times \exp \left(\mathrm{i} \pi \int_{M^{5}} \frac{1}{2} \tilde{w}_{1}(T M)\right.$ $\mathcal{P}(B))=1$. This boils down to

$$
\left\langle X U_{(\mathbf{i})} U_{(\mathbf{i i})}\right\rangle=\left.\int[\mathcal{D} B][\mathcal{D} b] U_{(\mathbf{i})} U_{(\mathbf{i i})} \exp \left(\mathrm{i} \pi \int_{M^{5}} b \cup \delta B\right)\right|_{\tilde{w}_{1}(T M)=k \delta^{\perp}\left(V_{X}^{4}\right)} .
$$

(2) Integrating out $b$ yields

$$
\begin{gathered}
\delta B=\ell_{(\mathbf{i})} \delta^{\perp}\left(\Sigma_{U_{(\mathbf{i})}}^{2}\right)+\ell_{(\mathbf{i i})} \delta^{\perp}\left(\Sigma_{U_{(i \mathbf{i})}}^{2}\right), \\
B=\ell_{(\mathbf{i})} \delta^{\perp}\left(V_{U_{(\mathbf{i})}}^{3}\right)+\ell_{(\mathbf{i i})} \delta^{\perp}\left(V_{U_{(i \mathbf{i})}}^{3}\right) .
\end{gathered}
$$

(3) We finally integrate out $B$, from Eq. (5.19):

$$
\begin{aligned}
& \left\langle X U_{(\mathbf{i})} U_{(\mathbf{i i})}\right\rangle \\
& =\left.\int[\mathcal{D} B] \mathrm{e}^{\left(-\mathrm{i} \pi\left(\int_{M^{5}}\left(\tilde{w}_{1}(T M) B+\frac{1}{2} \tilde{w}_{1}(T M)\left(w_{2}(T M)+w_{1}(T M)^{2}\right)\right)\left(\ell_{(\mathbf{i})} \delta^{\perp}\left(V_{U_{(\mathbf{i})}}^{3}\right)+\ell_{(\mathbf{i i})} \delta^{\perp}\left(V_{U_{(\mathbf{i})}}^{3}\right)\right)\right)\right.}\right|_{\substack{\tilde{w}_{1}(T M)=k \delta^{\perp}\left(V^{4}\right) \\
B=\ell_{(\mathbf{i})} \delta^{\perp}\left(V_{U_{(\mathbf{i})}}^{3}\right)}}
\end{aligned}
$$

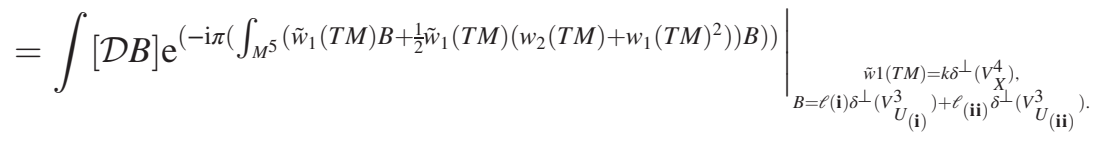

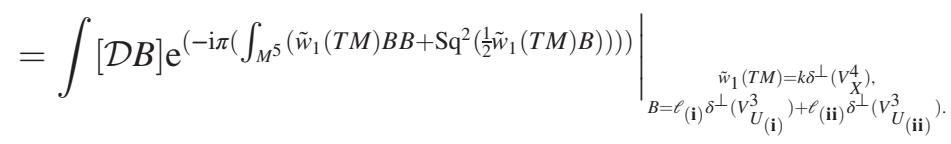

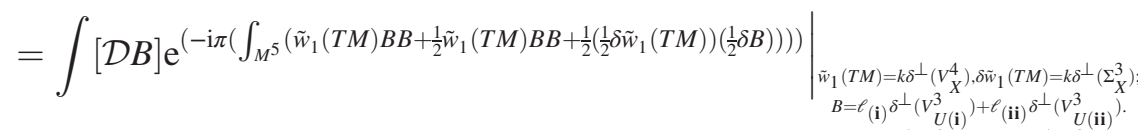

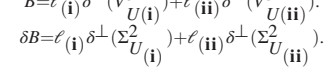

$$
\begin{aligned}
& =\mathrm{e}^{\left(-\mathrm{i} \pi\left(k \ell_{(\mathbf{i})} \ell_{(\mathbf{i i})} \cdot \#\left(V_{X}^{4} \cap V_{U_{1}}^{3} \cap V_{U_{2}}^{3}\right)+\frac{1}{\delta} \delta^{\perp}\left(\Sigma_{X}^{3}\right)\left(\delta^{\perp}\left(\Sigma_{U_{(i)}}^{2}\right)+\delta^{\perp}\left(\Sigma_{U_{(i i)}}^{2}\right)\right)\right)\right.} \quad \text { (self-intersecting \# terms) } \\
& \cong \mathrm{e}^{\left(-\mathrm{i} \pi\left(k \ell_{(\mathbf{i})} \ell_{(\mathbf{i})} \cdot \mathrm{Tlk}^{(5)}\left(\Sigma_{X}^{3}, \Sigma_{U_{(\mathbf{i})}^{2}}^{2}, \Sigma_{U_{(i \mathbf{i})}^{2}}^{2}\right)\right)\right.} .
\end{aligned}
$$


In Eq. (5.21), we used $\tilde{w}_{1}(T M)\left(w_{2}(T M)+\right.$ $\left.\left.w_{1}(T M)^{2}\right)\right) B=\tilde{w}_{1}(T M) u_{2} B=\operatorname{Sq}^{2}\left(\tilde{w}_{1}(T M) B\right)$. In Eq. (5.22), we rewrote $\frac{1}{2} \tilde{w}_{1}(T M) \mathcal{P}(B)$ via $^{40}$

$$
\begin{aligned}
& \frac{1}{2} \tilde{w}_{1}(T M) u_{2} B \\
& =\mathrm{Sq}^{2}\left(\frac{1}{2} \tilde{w}_{1}(T M) B\right) \\
& =\frac{1}{2} \tilde{w}_{1}(T M) B B+\mathrm{Sq}^{1}\left(\frac{1}{2} \tilde{w}_{1}(T M)\right) \mathrm{Sq}^{1} B \\
& =\frac{1}{2} \tilde{w}_{1}(T M) B B+\frac{1}{2}\left(\frac{1}{2} \delta \tilde{w}_{1}(T M)\right)\left(\frac{1}{2} \delta B\right) .
\end{aligned}
$$

We plugged all the constraints into the path integral Eq. (5.22) to obtain Eq. (5.23). ${ }^{41}$ We propose a setup to remove or renormalize the (self-intersecting \# terms) that appeared in Eq. (5.24), described in footnote 40. The second exponent in Eq. (5.23) shows that $\int_{M^{5}} \delta^{\perp}\left(\Sigma_{W}^{3}\right)\left(\delta^{\perp}\left(\Sigma_{U_{(i)}}^{2}\right)+\delta^{\perp}\left(\Sigma_{U_{(i i)}}^{2}\right)\right)=$ $\#\left(\Sigma_{X}^{3} \cap \Sigma_{U_{(i)}}^{2}\right)+\#\left(\Sigma_{X}^{3} \cap \Sigma_{U_{(i i)}}^{2}\right)$, which counts the number of intersections between our insertions of three-surface and two-surface. However, we choose by default that our insertions of three-surface and two-surface have no intersections (to avoid unnecessary singularities). Namely, we set \#( $\Sigma_{W}^{3} \cap$ $\left.\Sigma_{U_{(\mathbf{n})}}^{2}\right)=0$ for $(\mathbf{n})=(\mathbf{i})$ or $(\mathbf{i i})$, and $\#\left(\Sigma_{U_{(\mathbf{i})}}^{2} \cap\right.$
$\left.\Sigma_{U_{(i i)}}^{2}\right)=0$ by default. Overall, under the default assumption and the clarifications in footnote 40 , we obtain a final relation between Eq. (5.23) and our final effective answer Eq. (5.24). We use the congruence symbol $(\cong)$ to express that other unwanted terms can be removed by design.

In summary, we have derived the link invariant for the $5 \mathrm{~d}$ TQFT $\mathbf{Z}_{\mathrm{SET}_{\left(K_{1}=0, K_{2}=0\right)}}\left[M^{5}\right]$ in Eq. (5.24):

$\#\left(V_{X}^{4} \cap V_{U_{(\mathbf{i})}}^{3} \cap V_{U_{(i \mathrm{i})}}^{3}\right) \equiv \mathrm{Tlk}_{w_{1} B B}^{(5)}\left(\Sigma_{X}^{3}, \Sigma_{U_{(\mathrm{i})}}^{2}, \Sigma_{U_{(i \mathrm{i})}}^{2}\right)$.

The path integral, with appropriate insertions of extended operators, becomes Eq. (5.24) which provides the above link invariant.

$$
\text { B. } w_{1}(T M)^{3} B=w_{1}(T M)^{2} \mathrm{Sq}^{1} B
$$

\section{Version I: $w_{1}(T M)^{3} B$ and a quartic link invariant

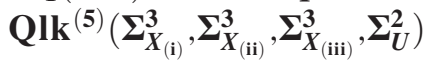

As a test example, we consider a $5 \mathrm{~d}$ TQFT obtained from summing over two-form field $B$ with the topological action $w_{1}(T M)^{3} B$ (i.e., gauging the one-form $\mathbb{Z}_{2}$ symmetry of this $5 \mathrm{~d}$ higher SPTs). For simplicity, we convert the cochain TQFT to a differential-form continuum TQFT. The partition function and the topological action of the gauged theory (see footnote 15) are

${ }^{40}$ We use the Cartan formula of the Steenrod square: $\operatorname{Sq}^{2}(u v)=\left(\operatorname{Sq}^{2} u\right) v+\left(\operatorname{Sq}^{1} u\right)\left(\operatorname{Sq}^{1} v\right)+u \operatorname{Sq}^{2}(v)$ where $u, v \in H^{*}(M, \mathbb{Z} 2)$.

${ }^{41}$ Here are some more explanations to derive Eq. (5.23):

(i) For $\left.\int[\mathcal{D} B] \mathrm{e}^{-\mathrm{i} \pi\left(\int_{M^{5}}\left(\tilde{w}_{1}(T M) B B\right)\right)}\right|_{\substack{\tilde{w}_{1}(T M)=k \delta^{\perp}\left(V_{X}^{4}\right) \\ B=\ell_{(i)} \delta^{\perp}\left(V_{U(\mathbf{i})}^{3}\right)+\ell_{(i i)} \delta^{\perp}\left(V_{U_{(i)}^{3}}^{3}\right)}}$, we get a mutual-quadratic crossing term $V_{U_{(\mathbf{i})}}^{3} \cap V_{U_{(i i)}}^{3}$ with a multiple $2 \pi$ exponent in $\mathrm{e}^{\mathrm{i} 2 \pi \#\left(V_{X}^{4} \cap V_{U_{(i)}}^{3} \cap V_{U_{(i i)}^{3}}^{3}\right)}$ which does not contribute to the expectation value. There are also two self-quadratic terms $V_{U_{(\mathbf{n})}}^{3} \cap V_{U_{(\mathbf{n})}}^{3}$ for $(\mathbf{n})=(\mathbf{i})$ or $(\mathbf{i i})$. These self-quadratic terms contribute, in principle, infinite many intersecting numbers in $\#\left(V_{X}^{4} \cap V_{U_{(\mathbf{n})}}^{3} \cap V_{U_{(\mathbf{n})}}^{3}\right)$ for $(\mathbf{n})=(\mathbf{i})$ or (ii). Since a multiple $2 \pi$ exponent has zero contribution to the expectation value, therefore either we can design an even but infinite number of points on each of \# $\left(V_{X}^{4} \cap V_{U_{(\mathbf{n})}}^{3} \cap V_{U_{(\mathbf{n})}}^{3}\right)$ for $(\mathbf{n})=(\mathbf{i})$ or $(\mathbf{i i})$ or we can absorb them into the (self-intersecting \# terms) in Eq. (5.23). In either case, this term does not have any net contribution in the end at Eq. (5.24).

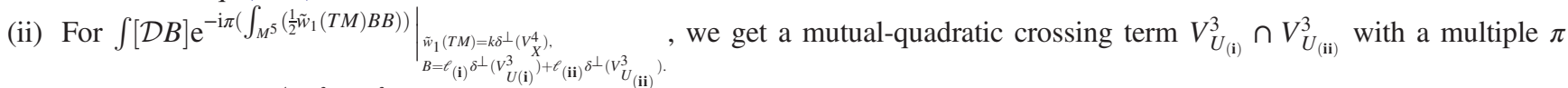
exponent in $\mathrm{e}^{\mathrm{i} \pi \#\left(V_{X}^{4} \cap V_{U_{(i)}}^{3} \cap V_{U_{(i i)}^{3}}^{3}\right)}$, which does contribute to the expectation value when this intersecting number \# is odd, in a 1 mod 2 effect. There are also two self-quadratic terms $V_{U_{(\mathbf{n})}}^{3} \cap V_{U_{(\mathbf{n})}}^{3}$ for $(\mathbf{n})=(\mathbf{i})$ or $(\mathbf{i i})$. Again either we can design a quadruple/ four-multiplet but infinite number of points for each of $\#\left(V_{X}^{4} \cap V_{U_{(\mathbf{n})}}^{3} \cap V_{U_{(\mathbf{n})}}^{3}\right)$ or we can absorb them into the (self-intersecting \# terms) in Eq. (5.23).

(iii) For $\left.\Sigma_{U_{(\mathbf{i})}}^{2} \int[\mathcal{D} B] \mathrm{e}^{\left(-\mathrm{i} \pi\left(\int_{M^{5}}\left(\frac{1}{8} \delta \tilde{\omega}_{1}(T M) \delta B\right)\right)\right)}\right|_{\substack{\delta \tilde{w}_{1}(T M)=k \delta^{\perp}\left(\Sigma_{X}^{3}\right) ; \\ \delta B=\ell_{(\mathbf{i})} \delta^{\perp}\left(\Sigma_{U_{(i)}}^{2}\right)+\ell_{(\mathbf{i i})} \delta^{\perp}\left(\Sigma_{U_{(i i)}}^{2}\right)}}=\mathrm{e}^{-\mathrm{i} \pi \int_{M^{5}}\left(\frac{1}{8}\left(k \delta^{\perp}\left(\Sigma_{X}^{3}\right)\right)\left(\ell_{(\mathbf{i})} \delta^{\perp}\left(\Sigma_{U_{(i)}}^{2}\right)+\ell_{(i i)} \delta^{\perp}\left(\Sigma_{U_{(i i)}}^{2}\right)\right)\right)}$, we find the exponent depends on the intersecting number $\#\left(\Sigma_{X}^{3} \cap \Sigma_{U_{(\mathbf{n})}}^{2}\right)$ for $(\mathbf{n})=(\mathbf{i})$ or $(\mathbf{i i})$, between three-surface and two-surface in a five manifold-although generically this number \# $\left(\Sigma_{X}^{3} \cap \Sigma_{U_{(\mathbf{n})}}^{2}\right)$ is finite but can be nonzero, we design by default that there is no intersection between any of our insertions of three-surface and two-surface into the path integral. Thus we set \# $\left(\Sigma_{X}^{3} \cap \Sigma_{U_{(\mathbf{n})}}^{2}\right)=0$ by default. 


$$
\begin{gathered}
\mathbf{Z}=\int[\mathcal{D} B][\mathcal{D} b][\mathcal{D} c] \exp (\mathrm{i} \mathbf{S}), \\
\mathbf{S}=\pi \int_{M^{5}} c \mathrm{~d} w_{1}(T M)+b \mathrm{~d} B+w_{1}(T M)^{3} B .
\end{gathered}
$$

This 5d TQFT is distinct from any of four classes of $\mathbf{Z}_{\mathrm{SET}_{\left(K_{1}, K_{2}\right)}}$, but it still serves as a useful toy model.

We first specify the gauge transformations of various fields. Let us assume the gauge transformations take the following form (see footnote 36 ):

$$
\begin{aligned}
w_{1}(T M) & \rightarrow w_{1}(T M)+\mathrm{d} \alpha, \\
B & \rightarrow B+\mathrm{d} \beta, \\
c & \rightarrow c+\mathrm{d} \gamma+\lambda, \\
b & \rightarrow b+\mathrm{d} \zeta+\mu .
\end{aligned}
$$

The variation of action under the gauge transformations is

$$
\begin{aligned}
\mathbf{S} \rightarrow & \mathbf{S}+\pi \int_{M^{5}} \mathrm{~d} \gamma \mathrm{d} w_{1}(T M)+\lambda \mathrm{d} w_{1}(T M)+\mathrm{d} \zeta \mathrm{d} B+\mu \mathrm{d} B \\
& +\left(\mathrm{d} \alpha \mathrm{d} \alpha w_{1}(T M)+w_{1}(T M)^{2} \mathrm{~d} \alpha+\mathrm{d} \alpha \mathrm{d} \alpha \mathrm{d} \alpha\right) B \\
& +\left(w_{1}(T M)^{3}+\mathrm{d} \alpha \mathrm{d} \alpha w_{1}(T M)\right. \\
& \left.+w_{1}(T M)^{2} \mathrm{~d} \alpha+\mathrm{d} \alpha \mathrm{d} \alpha \mathrm{d} \alpha\right) \mathrm{d} \beta \\
= & \mathbf{S}+\pi \int_{M^{5}} \lambda \mathrm{d} w_{1}(T M)+\mu \mathrm{d} B \\
& +\left(\alpha \mathrm{d} \alpha B \mathrm{~d} w_{1}(T M)-\alpha \mathrm{d} \alpha w_{1}(T M) \mathrm{d} B\right) \\
& -\alpha w_{1}(T M)^{2} \mathrm{~d} B-\alpha \mathrm{d} \alpha \mathrm{d} \alpha \mathrm{d} B \\
& +w_{1}(T M)^{2} \beta \mathrm{d} w_{1}(T M)+\alpha \mathrm{d} \alpha \mathrm{d} \beta \mathrm{d} w_{1}(T M),
\end{aligned}
$$

where we have used integration by part. For a closed five manifold without boundary, after integration by part we drop the total derivative terms $\mathrm{d}(\cdots)$ which have no effect on a closed five manifold without boundary. The gauge variance of the action, i.e., $\Delta \mathbf{S}=0$, requires

$$
\begin{aligned}
& \lambda=-\alpha \mathrm{d} \alpha B-w_{1}(T M)^{2} \beta-\alpha \mathrm{d} \alpha \mathrm{d} \beta, \\
& \mu=\alpha \mathrm{d} \alpha w_{1}(T M)+\alpha w_{1}(T M)^{2}+\alpha \mathrm{d} \alpha \mathrm{d} \alpha .
\end{aligned}
$$

The gauge invariant three-submanifold operator is

$$
\begin{aligned}
X & =\exp \left(\mathrm{i} \pi k\left(\int_{\Sigma^{3}} c+\int_{V^{4}} w_{1}(T M)^{2} B\right)\right) \\
& =\exp \left(\mathrm{i} \pi k\left(\int_{M^{5}}\left(\delta^{\perp}\left(\Sigma^{3}\right) c+\delta^{\perp}\left(V^{4}\right) w_{1}(T M)^{2} B\right)\right)\right)
\end{aligned}
$$

and the gauge invariant two-surface operator is

$$
\begin{aligned}
U & =\exp \left(\mathrm{i} \pi \ell\left(\int_{\Sigma^{2}} b-\int_{V^{3}} w_{1}(T M)^{3}\right)\right) \\
& =\exp \left(\mathrm{i} \pi \ell\left(\int_{M^{5}}\left(\delta^{\perp}\left(\Sigma^{2}\right) b-\delta^{\perp}\left(V^{3}\right) w_{1}(T M)^{3}\right)\right)\right),
\end{aligned}
$$

where $k, \ell \in \mathbb{Z}_{2}$. To verify the gauge invariance, we need to use $\mathrm{d} w_{1}(T M)=\mathrm{d} B=0$ on the two-surfaces and threesubmanifolds.

To compute the link invariants, we insert $X_{(\mathbf{i})}, X_{(\mathbf{i i})}$, $X_{(i i i)}, U$ into the path integral $\mathbf{Z}$. In the continuum field theory formulation, the link invariant is

$$
\begin{aligned}
& \left\langle X_{(\mathbf{i})} X_{(\mathbf{i i})} X_{(\mathbf{i i i})} U\right\rangle \\
& =\int[\mathcal{D} B][\mathcal{D} c][\mathcal{D} b] X_{(\mathbf{i})} X_{(\mathbf{i i})} X_{(\mathbf{i i i})} U \exp (\mathbf{i S}) \\
& =\int[\mathcal{D} B][\mathcal{D} c][\mathcal{D} b] X_{(\mathbf{i})} X_{(\mathbf{i i})} X_{(\mathbf{i i i})} U \\
& \quad \times \exp \left(\mathrm{i} \pi \int_{M^{5}} c \mathrm{~d} w_{1}(T M)+b \mathrm{~d} B+w_{1}(T M)^{3} B\right) .
\end{aligned}
$$

We compute $\left\langle X_{(\mathbf{i})} X_{(\mathbf{i i})} X_{(\mathbf{i i i})} U\right\rangle$ as follows:

(1) Integrating out $c$, we get

$$
\begin{aligned}
\mathrm{d} w_{1}(T M)= & k_{(\mathbf{i})} \delta^{\perp}\left(\Sigma_{X_{(i)}}^{3}\right)+k_{(\mathbf{i i})} \delta^{\perp}\left(\Sigma_{X_{(i i)}}^{3}\right) \\
& +k_{(\mathbf{i i i})} \delta^{\perp}\left(\Sigma_{X_{(i i i)}}^{3}\right), \\
w_{1}(T M)= & k_{(\mathbf{i})} \delta^{\perp}\left(V_{X_{(\mathbf{i})}}^{4}\right)+k_{(\mathbf{i i})} \delta^{\perp}\left(V_{X_{(i i)}}^{4}\right) \\
& +k_{(\mathbf{i i i})} \delta^{\perp}\left(V_{X_{(i i i)}}^{4}\right) .
\end{aligned}
$$

With the above configuration constraint, we get the double-counting mod 2 cancellation in the exponent of $\exp \left(\mathrm{i} \pi\left(\int_{M^{5}} w_{1}(T M)^{2} B\left(k_{(\mathbf{i})} \delta^{\perp}\left(V_{X_{(\mathbf{i})}}^{4}\right)+\right.\right.\right.$

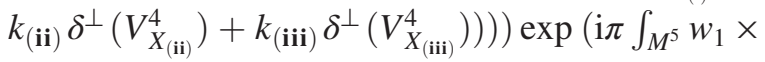
$\left.(T M)^{3} B\right)=1$. Consequently the link invariant boils down to

$$
\left\langle X_{(\mathbf{i})} X_{(\mathbf{i i})} X_{(\mathbf{i i i})} U\right\rangle=\left.\int[\mathcal{D} B][\mathcal{D} b] U \exp \left(\mathrm{i} \pi \int_{M^{5}} b \mathrm{~d} B\right)\right|_{w_{1}(T M)=k_{(\mathbf{i})} \delta^{\perp}\left(V_{X_{(i)}}^{4}\right)+k_{(\mathbf{i i})} \delta^{\perp}\left(V_{X_{(\mathbf{i i})}^{4}}^{4}\right)+k_{(\mathbf{i i i})} \delta^{\perp}\left(V_{X_{(i i i)}}^{4}\right)}
$$


(2) Integrating out $b$ further yields the constraint

$$
\begin{aligned}
\mathrm{d} B & =\ell \delta^{\perp}\left(\Sigma_{U}^{2}\right), \\
B & =\ell \delta^{\perp}\left(V_{U}^{3}\right) .
\end{aligned}
$$

(3) We finally integrate out $B$ as follows:

$$
\begin{aligned}
& \left\langle X_{(\mathbf{i})} X_{(\mathbf{i i})} X_{(\mathbf{i i i})} U\right\rangle
\end{aligned}
$$

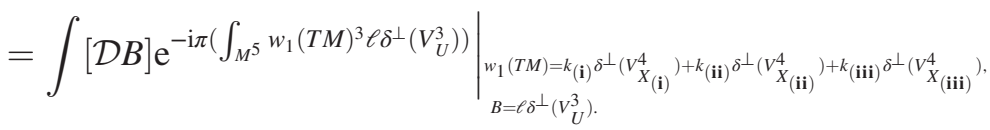

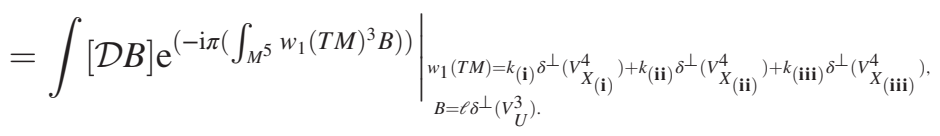

$$
\begin{aligned}
& =\mathrm{e}^{\left(-\mathrm{i} \pi\left(k _ { ( \mathbf { i } ) } k _ { ( \mathbf { i i } ) } k _ { ( \mathrm { iii } ) } \ell \left(\#\left(V_{X_{(i)}}^{4} \cap V_{X_{(i i)}}^{4} \cap V_{X_{(i i i)}}^{4} \cap V_{U}^{3}\right)+\#\left(V_{X_{(i i)}}^{4} \cap V_{X_{(i i i)}}^{4} \cap V_{X_{(i)}}^{4} \cap V_{U}^{3}\right)+\#\left(V_{X_{(i i i)}^{4}}^{4} \cap V_{X_{(i)}}^{4} \cap V_{X_{(i i)}}^{4} \cap V_{U}^{3}\right)\right.\right.\right.} \\
& \left.\left.\left.\#\left(V_{X_{(i)}}^{4} \cap V_{X_{(i i i)}}^{4} \cap V_{X_{(i i)}}^{4} \cap V_{U}^{3}\right)+\#\left(V_{X_{(i i i)}}^{4} \cap V_{X_{(i i)}}^{4} \cap V_{X_{(i)}}^{4} \cap V_{U}^{3}\right)+\#\left(V_{X_{(i i)}}^{4} \cap V_{X_{(i)}}^{4} \cap V_{X_{(i i i)}}^{4} \cap V_{U}^{3}\right)\right)\right)\right) \\
& \cdot(\cdots) \cdot(\text { self-intersecting \# terms) } \\
& \cong \mathrm{e}^{\left(-\mathrm{i} \pi\left(k_{(\mathrm{i})} k_{(\mathrm{iii})} k_{(\mathrm{iii})} \ell \cdot 6 \#\left(V_{X_{(\mathrm{i})}}^{4} \cap V_{X_{(\mathrm{ii})}^{4}}^{4} \cap V_{X_{(\mathrm{iii})}}^{4} \cap V_{U}^{3}\right)\right)\right)} \cdot(\cdots)
\end{aligned}
$$

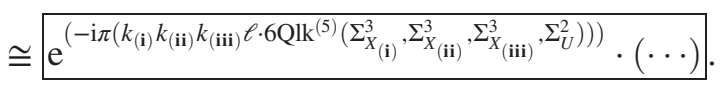

We propose a setup to remove or renormalize the (self-intersecting \# terms) that appeared in Eq. (5.39), following the same strategy in footnote 40.

For $\mathbf{S}=\pi \int_{M^{5}} c \mathrm{~d} w_{1}(T M)+b \mathrm{~d} B+w_{1}(T M)^{3} B$, we derive the link invariant for the $5 \mathrm{~d}$ TQFT $\mathbf{Z}_{\mathrm{SET}}\left[M^{5}\right]$ in Eq. (5.39) and Eq. (5.40):

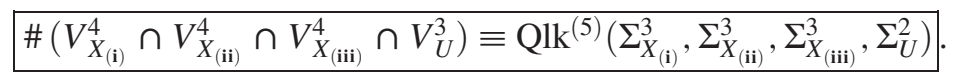

The path integral with appropriate extended operator insertions becomes Eq. (5.40) which provides the above link invariant. However, note that the factorial $3 !=6$ trivializes the complex $\mathrm{e}^{\mathrm{i} \pi}$ phase to $\mathrm{e}^{\mathrm{i} 6 \pi}$. It may be possible to take into account (see footnote 38) from the subtle graded noncommutativity of the cochain field effect. Thus one may need to go beyond the continuum differential form TQFT formulation by using the cochain TQFT formulation in order to see the subleading effect.

\section{Version II: $w_{1}(T M)^{2} \mathrm{Sq}^{1} B$ and a triple link invariant $\operatorname{Tlk}_{w_{1} w_{1} \mathrm{~d} B}^{(5)}\left(\Sigma_{X_{(\mathrm{i})}}^{3}, \Sigma_{X_{(i \mathrm{i})}}^{3}, \Sigma_{U}^{2}\right)$}

As another test example, we consider a $5 \mathrm{~d}$ TQFT obtained from summing over two-form field $B$ with the topological action $w_{1}(T M)^{2} \mathrm{Sq}^{1} B$. We again use the continuum version of the TQFT. ${ }^{42}$ Its partition function and the topological action (see footnote 15) are

$$
\begin{gathered}
\mathbf{Z}=\int[\mathcal{D} B][\mathcal{D} b][\mathcal{D} c] \exp (\mathrm{i} \mathbf{S}), \\
\mathbf{S}=\pi \int_{M^{5}} c \mathrm{~d} w_{1}(T M)+b \mathrm{~d} B+w_{1}(T M)^{2} \mathrm{Sq}^{1} B,
\end{gathered}
$$

\footnotetext{
${ }^{42}$ Even though $w_{1}(T M)^{2} \mathrm{Sq}^{1} B$ is a rewriting of $w_{1}(T M)^{3} B$ on a closed five manifold, it turns out that we still gain new insights about an additional link invariant.
} 
$\mathbf{S}=\pi \int_{M^{5}} c \mathrm{~d} w_{1}(T M)+b \mathrm{~d} B+w_{1}(T M)^{2} \frac{1}{2} \mathrm{~d} B$.

We assume the gauge transformations have the following ansatz:

$$
\begin{aligned}
w_{1}(T M) & \rightarrow w_{1}(T M)+\mathrm{d} \alpha, \\
B & \rightarrow B+\mathrm{d} \beta, \\
c & \rightarrow c+\mathrm{d} \gamma+\lambda, \\
b & \rightarrow b+\mathrm{d} \zeta+\mu .
\end{aligned}
$$

Under the gauge transformations, the action transforms as

$$
\begin{aligned}
\mathbf{S} \rightarrow & \mathbf{S}+\pi \int_{M^{5}} \mathrm{~d} \gamma \mathrm{d} w_{1}(T M)+\lambda \mathrm{d} w_{1}(T M)+\mathrm{d} \zeta \mathrm{d} B+\mu \mathrm{d} B \\
& +\left(w_{1}(T M) \mathrm{d} \alpha+\mathrm{d} \alpha w_{1}(T M)+\mathrm{d} \alpha \mathrm{d} \alpha\right) \frac{1}{2} \mathrm{~d} B \\
& +\left(w_{1}(T M)^{2}+w_{1}(T M) \mathrm{d} \alpha+\mathrm{d} \alpha w_{1}(T M)\right. \\
& +\mathrm{d} \alpha \mathrm{d} \alpha) \frac{1}{2} \mathrm{~d}^{2} \beta \\
= & \mathbf{S}+\pi \int_{M^{5}} \lambda \mathrm{d} w_{1}(T M)+\mu \mathrm{d} B \\
& +\frac{1}{2}\left(w_{1}(T M) \mathrm{d} \alpha+\mathrm{d} \alpha w_{1}(T M)+\mathrm{d} \alpha \mathrm{d} \alpha\right) \mathrm{d} B
\end{aligned}
$$

where we have used integration by part. $\Delta \mathbf{S}=0$ requires

$$
\begin{aligned}
\lambda & =0, \\
\mu & =-\frac{1}{2}\left(w_{1}(T M) \mathrm{d} \alpha+\mathrm{d} \alpha w_{1}(T M)+\mathrm{d} \alpha \mathrm{d} \alpha\right) .
\end{aligned}
$$

The gauge invariant three-submanifold operator is

$$
\begin{aligned}
X & =\exp \left(\mathrm{i} \pi k\left(\int_{\Sigma^{3}} c\right)\right) \\
& =\exp \left(\mathrm{i} \pi k\left(\int_{M^{5}}\left(\delta^{\perp}\left(\Sigma^{3}\right) c\right)\right)\right)
\end{aligned}
$$

and the gauge invariant two-surface operator is

$$
\begin{aligned}
U & =\exp \left(\mathrm{i} \pi \ell\left(\int_{\Sigma^{2}}\left(b+\frac{1}{2} w_{1}(T M)^{2}\right)\right)\right) \\
& =\exp \left(\mathrm{i} \pi \ell\left(\int_{M^{5}}\left(\delta^{\perp}\left(\Sigma^{2}\right)\left(b+\frac{1}{2} w_{1}(T M)^{2}\right)\right)\right)\right.
\end{aligned}
$$

where $k, \ell \in \mathbb{Z}_{2}$.

We proceed to compute the link invariants by inserting $X_{(\mathbf{i})}, X_{(\mathbf{i i})}, U$ into the path integral $\mathbf{Z}$,

$$
\begin{aligned}
\left\langle X_{(\mathbf{i})} X_{(\mathbf{i i})} U\right\rangle & =\int[\mathcal{D} B][\mathcal{D} c][\mathcal{D} b] X_{(\mathbf{i})} X_{(\mathbf{i i})} U \exp (\mathbf{i} \mathbf{S}), \\
\left\langle X_{(\mathbf{i})} X_{(\mathbf{i i})} U\right\rangle & =\int[\mathcal{D} B][\mathcal{D} c][\mathcal{D} b] X_{(\mathbf{i})} X_{(\mathbf{i i})} U \exp \left(\mathrm{i} \pi \int_{M^{5}} c \mathrm{~d} w_{1}(T M)+b \mathrm{~d} B+w_{1}(T M)^{2} \frac{1}{2} \mathrm{~d} B\right) .
\end{aligned}
$$

To evaluate $\left\langle X_{(\mathbf{i})} X_{(\mathbf{i i})} U\right\rangle$, we integrate out various fields step by step:

(1) Integrating out $c$, we get

$$
\begin{aligned}
\mathrm{d} w_{1}(T M) & =k_{(\mathbf{i})} \delta^{\perp}\left(\Sigma_{X_{(\mathbf{i})}}^{3}\right)+k_{(\mathbf{i i})} \delta^{\perp}\left(\Sigma_{X_{(\mathbf{i})}}^{3}\right) \\
w_{1}(T M) & =k_{(\mathbf{i})} \delta^{\perp}\left(V_{X_{(\mathbf{i})}}^{4}\right)+k_{(\mathbf{i i})} \delta^{\perp}\left(V_{X_{(i \mathbf{i})}}^{4}\right)
\end{aligned}
$$

The link invariant thus boils down to

$$
\left\langle X_{(\mathbf{i})} X_{(\mathbf{i i})} U\right\rangle=\left.\int[\mathcal{D} B][\mathcal{D} b] U \exp \left(\mathrm{i} \pi \int_{M^{5}} b \mathrm{~d} B+w_{1}(T M)^{2} \frac{1}{2} \mathrm{~d} B\right)\right|_{w_{1}(T M)=k_{(\mathbf{i})} \delta^{\perp}\left(V_{X_{(i)}}^{4}\right)+k_{(\mathbf{i i})} \delta^{\perp}\left(V_{X_{(i \mathbf{i})}}^{4}\right)}
$$

(2) Integrate out $b$, we get the constraint

$$
\begin{aligned}
\mathrm{d} B & =\ell \delta^{\perp}\left(\Sigma_{U}^{2}\right), \\
B & =\ell \delta^{\perp}\left(V_{U}^{3}\right) .
\end{aligned}
$$


(3) We finally integrate out $B$ in Eq. (5.54):

$$
\begin{aligned}
& \left\langle X_{(\mathbf{i})} X_{(\mathbf{i i})} U\right\rangle
\end{aligned}
$$

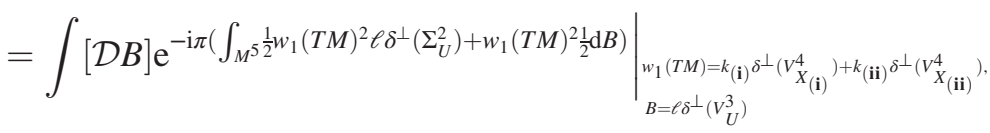

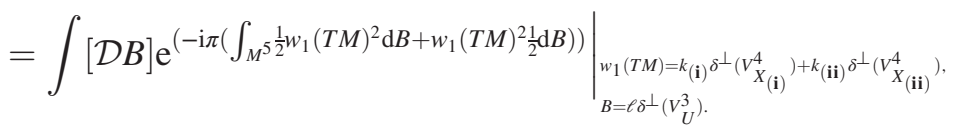

$$
\begin{aligned}
& =\mathrm{e}^{\left(-\mathrm{i} \pi\left(k_{(\mathbf{i})} k_{(\mathbf{i i})} \ell \ell\left(\#\left(V_{X_{(i)}}^{4} \cap V_{X_{(i \mathbf{i})}^{4}}^{4} \cap \Sigma_{U}^{2}\right)+\#\left(V_{X_{(i \mathbf{i})}^{4}}^{4} \cap V_{X_{(i)}}^{4} \cap \Sigma_{U}^{2}\right)\right)\right)\right)} \\
& \cdot(\cdots) \cdot(\text { self-intersecting \# terms) } \\
& \cong \mathrm{e}^{\left(-\mathrm{i} \pi\left(k_{(\mathbf{i})} k_{(\mathbf{i})} \ell \cdot\left(\mathrm{Tlk}_{w_{1} w_{1} \mathrm{~d} B}^{(5)}\left(\Sigma_{X_{(\mathbf{i})}}^{3}, \Sigma_{X_{(i \mathbf{i})}}^{3}, \Sigma_{U}^{2}\right)+\mathrm{Tlk}_{w_{1} w_{1} \mathrm{~d} B}^{(5)}\left(\Sigma_{X_{(\mathbf{i i})}}^{3}, \Sigma_{X_{(\mathbf{i})}}^{3}, \Sigma_{U}^{2}\right)\right)\right)\right.} \cdot(\cdots) .
\end{aligned}
$$

We propose a setup to remove or renormalize the (self-intersecting \# terms) that appeared in Eq. (5.57), following the same strategy as footnote 40 .

For $\mathbf{S}=\pi \int_{M^{5}} c \mathrm{~d} w_{1}(T M)+b \mathrm{~d} B+w_{1}(T M)^{2} \frac{1}{2} \mathrm{~d} B$, we derive the link invariant for the $5 \mathrm{~d}$ TQFT $\mathbf{Z}_{\mathrm{SET}}\left[M^{5}\right]$ in Eq. (5.57) and Eq. (5.58):

$$
\#\left(V_{X_{(i)}}^{4} \cap V_{X_{(i)}}^{4} \cap \Sigma_{U}^{2}\right) \equiv \operatorname{Tlk}_{w_{1} w_{1} \mathrm{~d} B}^{(5)}\left(\Sigma_{X_{(i)}}^{3}, \Sigma_{X_{(i i)}}^{3}, \Sigma_{U}^{2}\right) .
$$

The path integral with appropriate extended operator insertions become Eq. (5.58) which provides the above link invariant. However, note that the two terms on the exponent of Eq. (5.58) are the same, which trivializes the complex $\mathrm{e}^{\mathrm{i} \pi}$ to $\mathrm{e}^{\mathrm{i} 2 \pi}$. It may be possible to take into account (see footnote 38) from the subtle graded noncommutativity of the cochain field effect. Thus one may need to go beyond the continuum differential form TQFT formulation by using the cochain TQFT formulation in order to see the subleading effect.

\section{C. $w_{3}(T M) B=w_{2}(T M) S^{1} B$ and a quadratic link invariant $\mathbf{L} \mathbf{k}_{w_{2} \mathrm{~d} B}^{(\mathbf{5})}\left(\Sigma_{U^{\prime}}^{2}, \Sigma_{U}^{2}\right)$}

We further consider a $5 \mathrm{~d}$ TQFT obtained from summing over two-form field $B$ of $w_{3}(T M) B=w_{2}(T M) \mathrm{Sq}^{1} B$. We again adopt the continuum version of TQFT. The partition function and action (see footnote 15) are

$$
\begin{gathered}
\mathbf{Z}=\int[\mathcal{D} B][\mathcal{D} b][\mathcal{D} h] \exp (\mathbf{i} \mathbf{S}), \\
\mathbf{S}=\pi \int_{M^{5}} h \mathrm{~d} w_{2}(T M)+b \mathrm{~d} B+w_{2}(T M) \operatorname{Sq}^{1} B \\
\mathbf{S}=\pi \int_{M^{5}} h \mathrm{~d} w_{2}(T M)+b \mathrm{~d} B+w_{2}(T M) \frac{1}{2} \mathrm{~d} B .
\end{gathered}
$$

We assume the gauge transformations have the following ansatz:

$$
\begin{aligned}
w_{2}(T M) & \rightarrow w_{2}(T M)+\mathrm{d} \alpha, \\
B & \rightarrow B+\mathrm{d} \beta, \\
h & \rightarrow h+\mathrm{d} \gamma+\lambda, \\
b & \rightarrow b+\mathrm{d} \zeta+\mu .
\end{aligned}
$$

Under the gauge transformation, the action transforms as

$$
\begin{aligned}
\mathbf{S} \rightarrow & \mathbf{S}+\pi \int_{M^{5}} \mathrm{~d} \gamma \mathrm{d} w_{2}(T M)+\lambda \mathrm{d} w_{2}(T M)+\mathrm{d} \zeta \mathrm{d} B+\mu \mathrm{d} B \\
& +\mathrm{d} \alpha \frac{1}{2} \mathrm{~d} B+w_{2}(T M) \frac{1}{2} \mathrm{~d}^{2} \beta+\mathrm{d} \alpha \frac{1}{2} \mathrm{~d}^{2} \beta \\
= & \mathbf{S}+\pi \int_{M^{5}} \lambda \mathrm{d} w_{2}(T M)+\mu \mathrm{d} B+\left(\frac{1}{2} \mathrm{~d} \alpha\right) \mathrm{d} B \\
& +\left(-\frac{1}{2} \mathrm{~d} \beta\right) \mathrm{d} w_{2}(T M) .
\end{aligned}
$$

Thus $\Delta \mathbf{S}=0$ requires 


$$
\begin{aligned}
& \lambda=\left(\frac{1}{2} \mathrm{~d} \beta\right), \\
& \mu=-\left(\frac{1}{2} \mathrm{~d} \alpha\right) .
\end{aligned}
$$

There are two types of gauge invariant two-surface operators,

$$
\begin{aligned}
U^{\prime} & =\exp \left(\mathrm{i} \pi k\left(\int_{\Sigma^{2}} h-\int_{V^{3}} \frac{1}{2} \mathrm{~d} B\right)\right) \\
& =\exp \left(\mathrm{i} \pi k\left(\int_{M^{5}}\left(\delta^{\perp}\left(\Sigma^{2}\right) h-\delta^{\perp}\left(V^{3}\right) \frac{1}{2} \mathrm{~d} B\right)\right)\right) \\
& =\exp \left(\mathrm{i} \pi k\left(\int_{M^{5}}\left(\delta^{\perp}\left(\Sigma^{2}\right)\left(h-\frac{1}{2} B\right)\right)\right)\right)
\end{aligned}
$$

and

$$
\begin{aligned}
U & =\exp \left(\mathrm{i} \pi \ell\left(\int_{\Sigma^{2}} b+\int_{V^{3}} \frac{1}{2} \mathrm{~d} w_{2}(T M)\right)\right) \\
& =\exp \left(\mathrm{i} \pi \ell\left(\int_{\Sigma^{2}} b+\int_{\Sigma^{2}} \frac{1}{2} w_{2}(T M)\right)\right) \\
& =\exp \left(\mathrm{i} \pi \ell\left(\int_{M^{5}} \delta^{\perp}\left(\Sigma^{2}\right)\left(b+\frac{1}{2} w_{2}(T M)\right)\right)\right),
\end{aligned}
$$

where $k, \ell \in \mathbb{Z}_{2}$.

We define the link invariant by inserting $U^{\prime}, U$ into the path integral $\mathbf{Z}$

$$
\begin{aligned}
\left\langle U^{\prime} U\right\rangle= & \int[\mathcal{D} B][\mathcal{D} h][\mathcal{D} b] U^{\prime} U \exp \\
& \times\left(\mathrm{i} \pi \int_{M^{5}} h \mathrm{~d} w_{2}(T M)+b \mathrm{~d} B+w_{2}(T M) \frac{1}{2} \mathrm{~d} B\right) .
\end{aligned}
$$

Below we evaluate $\left\langle U^{\prime} U\right\rangle$ by integrating out various fields:

(1) Integrating out $h$, we get

$$
\begin{aligned}
\mathrm{d} w_{2}(T M) & =k \delta^{\perp}\left(\Sigma_{U^{\prime}}^{2}\right), \\
w_{2}(T M) & =k \delta^{\perp}\left(V_{U^{\prime}}^{3}\right)
\end{aligned}
$$

Plugging the above constraints into the partition function, we find the double-counting mod 2 cancellation in the exponent of $\exp \left(\mathrm{i} \pi\left(\int_{M^{5}} \delta^{\perp}\left(V_{U^{\prime}}^{3}\right) \times\right.\right.$ $\left.\left.\frac{k}{2} \mathrm{~d} B+w_{2}(T M) \frac{1}{2} \mathrm{~d} B\right)\right)=1$. Thus the link invariant boils down to

$$
\begin{aligned}
\left\langle U^{\prime} U\right\rangle= & \int[\mathcal{D} B][\mathcal{D} b] U \exp \\
& \times\left.\left(\mathrm{i} \pi \int_{M^{5}} b \mathrm{~d} B\right)\right|_{w_{2}(T M)=k \delta^{\perp}\left(V_{U^{\prime}}^{3}\right)}
\end{aligned}
$$

(2) Integrating out $b$, we get the constraint

$$
\begin{aligned}
\mathrm{d} B & =\ell \delta^{\perp}\left(\Sigma_{U}^{2}\right), \\
B & =\ell \delta^{\perp}\left(V_{U}^{3}\right) .
\end{aligned}
$$

(3) We finally integrate out $B$ in Eq. (5.71):

$$
\begin{aligned}
& \left\langle U^{\prime} U\right\rangle
\end{aligned}
$$

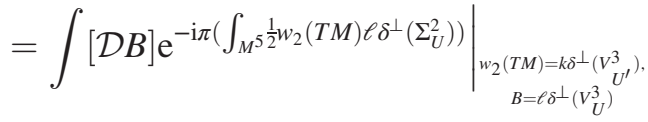

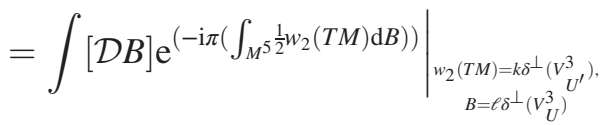

$$
\begin{aligned}
& =\mathrm{e}^{\left(-\mathrm{i} \pi\left(\frac{k \ell}{2} \cdot \#\left(V_{U^{\prime}}^{3} \cap \Sigma_{U}^{2}\right)\right)\right)}
\end{aligned}
$$

$$
\cong \mathrm{e}^{\left(-\mathrm{i} \pi\left(\frac{k \ell}{2} \cdot \operatorname{Lk}^{(5)}\left(\Sigma_{U^{\prime}}^{2}, \Sigma_{U}^{2}\right)\right)\right)} \text {. }
$$

We derive the link invariant for the $5 \mathrm{~d}$ TQFT $\mathbf{Z}_{\mathrm{SET}}\left[M^{5}\right]$ for $\mathbf{S}=\pi \int_{M^{5}} h \mathrm{~d} w_{2}(T M)+b \mathrm{~d} B+w_{2}(T M) \mathrm{Sq}^{1} B$ in Eq. (5.75):

$$
\#\left(V_{U^{\prime}}^{3} \cap \Sigma_{U}^{2}\right) \equiv \mathrm{Lk}_{w_{2} \mathrm{~d} B}^{(5)}\left(\Sigma_{U^{\prime}}^{2}, \Sigma_{U}^{2}\right)
$$

The path integral with appropriate extended operators insertions become Eq. (5.75) which provides the above link invariant.

\section{D. $B \mathrm{Sq}^{1} B+\left(1+K_{1}\right) w_{1}(T M)^{2} \mathrm{Sq}^{1} B+w_{2}(T M) \mathrm{Sq}^{1} B$ and more link invariants: $\mathbf{T l k}_{w_{1} w_{1} \mathrm{~d} B}^{(5)}\left(\Sigma_{X_{(\mathrm{i})}}^{3}, \Sigma_{X_{(\mathrm{ii})}}^{3}, \Sigma_{U}^{2}\right)$,

$$
\mathbf{L} \mathbf{k}_{B \mathrm{~d} B}^{(\mathbf{5})}\left(\boldsymbol{\Sigma}_{U_{(\mathrm{i})}^{2}}^{\mathbf{2}}, \boldsymbol{\Sigma}_{U_{(\mathrm{ii})}}^{2}\right) \text {, and } \mathbf{L} \mathbf{k}_{w_{2} \mathrm{~d} B}^{(5)}\left(\boldsymbol{\Sigma}_{U^{\prime}}^{2}, \Sigma_{U}^{2}\right)
$$

We finally consider the generic form including the four classes of $\mathbf{Z}_{\mathrm{SET}_{\left(K_{1}, K_{2}\right)}^{5 \mathrm{~d}}}$ in Eq. (4.5) by gauging $\mathbf{Z}_{\mathrm{SPT}_{\left(K_{1}, K_{2}\right)}}^{\mathrm{dd}}$ in Eq. (2.56), with $\left(K_{1}, K_{2}\right) \in\left(\mathbb{Z}_{2}, \mathbb{Z}_{2}\right)$ labeling the four siblings. Below, we find it convenient to introduce $K_{1}^{\prime}$ via $K_{1}^{\prime}:=1+K_{1} \bmod 2$.

The partition function and action (see footnote 15) are

$$
\mathbf{Z}=\int[\mathcal{D} B][\mathcal{D} b][\mathcal{D} h][\mathcal{D} c] \exp (\mathrm{iS})
$$

$$
\begin{aligned}
\mathbf{S}= & \pi \int_{M^{5}} K_{1}^{\prime} c \mathrm{~d} w_{1}(T M)+h \mathrm{~d}_{2}(T M)+b \mathrm{~d} B+B \mathrm{Sq}^{1} B \\
& +K_{1}^{\prime} w_{1}(T M)^{2} \mathrm{Sq}^{1} B+w_{2}(T M) \mathrm{Sq}^{1} B \\
\mathbf{S}= & \pi \int_{M^{5}} K_{1}^{\prime} c \mathrm{~d} w_{1}(T M)+h \mathrm{~d} w_{2}(T M)+b \mathrm{~d} B+B \frac{1}{2} \mathrm{~d} B \\
& +K_{1}^{\prime} w_{1}(T M)^{2} \frac{1}{2} \mathrm{~d} B+w_{2}(T M) \frac{1}{2} \mathrm{~d} B .
\end{aligned}
$$




\section{Gauge invariance}

We assume the following ansatz of the gauge transformations:

$$
\begin{aligned}
w_{1}(T M) & \rightarrow w_{1}(T M)+\mathrm{d} \alpha_{1}, \\
w_{2}(T M) & \rightarrow w_{2}(T M)+\mathrm{d} \alpha_{2}, \\
B & \rightarrow B+\mathrm{d} \beta, \\
c & \rightarrow c+\mathrm{d} \gamma_{1}+\lambda_{1}, \\
h & \rightarrow h+\mathrm{d} \gamma_{2}+\lambda_{2}, \\
b & \rightarrow b+\mathrm{d} \zeta+\mu .
\end{aligned}
$$

The gauge variation of the action is

$$
\begin{aligned}
\mathbf{S} \rightarrow & \mathbf{S}+\pi \int_{M^{5}} K_{1}^{\prime} \mathrm{d} \gamma_{1} \mathrm{~d} w_{1}(T M)+K_{1}^{\prime} \lambda_{1} \mathrm{~d} w_{1}(T M)+\mathrm{d} \gamma_{2} \mathrm{~d} w_{2}(T M)+\lambda_{2} \mathrm{~d} w_{2}(T M) \\
& +\mathrm{d} \zeta \mathrm{d} B+\mu \mathrm{d} B+\mathrm{d} \beta \frac{1}{2} \mathrm{~d} B+B \frac{1}{2} \mathrm{~d}^{2} \beta+\mathrm{d} \beta \frac{1}{2} \mathrm{~d}^{2} \beta \\
& +K_{1}^{\prime}\left(w_{1}(T M) \mathrm{d} \alpha_{1}+\mathrm{d} \alpha_{1} w_{1}(T M)+\mathrm{d} \alpha_{1} \mathrm{~d} \alpha_{1}\right) \frac{1}{2} \mathrm{~d} B \\
& +K_{1}^{\prime}\left(w_{1}(T M)^{2}+w_{1}(T M) \mathrm{d} \alpha_{1}+\mathrm{d} \alpha_{1} w_{1}(T M)+\mathrm{d} \alpha_{1} \mathrm{~d} \alpha_{1}\right) \frac{1}{2} \mathrm{~d}^{2} \beta \\
& +\mathrm{d} \alpha_{2} \frac{1}{2} \mathrm{~d} B+w_{2}(T M) \frac{1}{2} \mathrm{~d}^{2} \beta+\mathrm{d} \alpha_{2} \frac{1}{2} \mathrm{~d}^{2} \beta \\
& \quad \mathbf{S}+\pi \int_{M^{5}} K_{1}^{\prime} \lambda_{1} \mathrm{~d} w_{1}(T M)+\lambda_{2} \mathrm{~d} w_{2}(T M)+\mu \mathrm{d} B+\left(\frac{1}{2} \mathrm{~d} \alpha_{2}\right) \mathrm{d} B+\left(-\frac{1}{2} \mathrm{~d} \beta\right) \mathrm{d} w_{2}(T M) \\
+ & K_{1}^{\prime} \frac{1}{2}\left(w_{1}(T M) \mathrm{d} \alpha_{1}+\mathrm{d} \alpha_{1} w_{1}(T M)+\mathrm{d} \alpha_{1} \mathrm{~d} \alpha_{1}\right) \mathrm{d} B,
\end{aligned}
$$

where we have used integration by part. Gauge invariance, i.e., $\Delta \mathbf{S}=0$, requires

$$
\begin{aligned}
K_{1}^{\prime} \lambda_{1} & =0 \\
\lambda_{2} & =\frac{1}{2} \mathrm{~d} \beta, \\
\mu & =-K_{1}^{\prime} \frac{1}{2}\left(w_{1}(T M) \mathrm{d} \alpha_{1}+\mathrm{d} \alpha_{1} w_{1}(T M)+\mathrm{d} \alpha_{1} \mathrm{~d} \alpha_{1}\right)-\frac{1}{2} \mathrm{~d} \alpha_{2} .
\end{aligned}
$$

\section{Extended two-surface/three-brane operators and link invariants}

The gauge invariant three-manifold operator is

$$
\begin{aligned}
X & =\exp \left(\mathrm{i} \pi k K_{1}^{\prime}\left(\int_{\Sigma^{3}} c\right)\right) \\
& =\exp \left(\mathrm{i} \pi k\left(1+K_{1}\right)\left(\int_{M^{5}}\left(\delta^{\perp}\left(\Sigma^{3}\right) c\right)\right)\right)
\end{aligned}
$$

$X$ is trivial when $K_{1}^{\prime}=1+K_{1}=0 \bmod 2$. 
There are two types of gauge invariant two-surface operators,

$$
\begin{aligned}
U^{\prime} & =\exp \left(\mathrm{i} \pi k^{\prime}\left(\int_{\Sigma^{2}} h-\int_{V^{3}} \frac{1}{2} \mathrm{~d} B\right)\right) \\
& =\exp \left(\mathrm{i} \pi k^{\prime}\left(\int_{M^{5}}\left(\delta^{\perp}\left(\Sigma^{2}\right) h-\delta^{\perp}\left(V^{3}\right) \frac{1}{2} \mathrm{~d} B\right)\right)\right) \\
& =\exp \left(\mathrm{i} \pi k^{\prime}\left(\int_{M^{5}}\left(\delta^{\perp}\left(\Sigma^{2}\right)\left(h-\frac{1}{2} B\right)\right)\right)\right) \\
& =\exp \left(\mathrm{i} \pi k^{\prime}\left(\int_{M^{5}}\left(\delta^{\perp}\left(\Sigma^{2}\right)\left(h-\frac{1}{2} B\right)\right)\right)\right)
\end{aligned}
$$

and

$$
\begin{aligned}
U & =\exp \left(\mathrm{i} \pi \ell\left(\int_{\Sigma^{2}}\left(b+K_{1}{ }^{\prime} \frac{1}{2} w_{1}(T M)^{2}+\frac{1}{2} w_{2}(T M)\right)\right)\right) \\
& =\exp \left(\mathrm{i} \pi \ell\left(\int_{M^{5}}\left(\delta^{\perp}\left(\Sigma^{2}\right)\left(b+K_{1}{ }^{\prime} \frac{1}{2} w_{1}(T M)^{2}+\frac{1}{2} w_{2}(T M)\right)\right)\right)\right) \\
& =\exp \left(\mathrm{i} \pi \ell\left(\int_{M^{5}}\left(\delta^{\perp}\left(\Sigma^{2}\right)\left(b+\left(1+K_{1}\right) \frac{1}{2} w_{1}(T M)^{2}+\frac{1}{2} w_{2}(T M)\right)\right)\right)\right),
\end{aligned}
$$

where $k, k^{\prime}, \ell \in \mathbb{Z}_{2}$.

Inserting $X_{(\mathbf{i})}, X_{(\mathbf{i i})}, U^{\prime}, U_{(\mathbf{i})}, U_{(\mathbf{i i})}$ into path integral $\mathbf{Z}$, we define the link invariant as

$$
\begin{aligned}
\left\langle X_{(\mathbf{i})} X_{(\mathbf{i i})} U^{\prime} U_{(\mathbf{i})} U_{(\mathbf{i i})}\right\rangle= & \int[\mathcal{D} B][\mathcal{D} b][\mathcal{D} h][\mathcal{D} c] X_{(\mathbf{i})} X_{(\mathbf{i i})} U^{\prime} U_{(\mathbf{i})} U_{(\mathbf{i i})} \exp (\mathbf{i} \mathbf{S}) \\
= & \int[\mathcal{D} B][\mathcal{D} b][\mathcal{D} h][\mathcal{D} c] X_{(\mathbf{i})} X_{(\mathbf{i i})} U^{\prime} U_{(\mathbf{i})} U_{(\mathbf{i i})} \exp \left(\mathrm{i} \pi \int_{M^{5}} K_{1}{ }^{\prime} c \mathrm{~d} w_{1}(T M)\right. \\
& \left.+h \mathrm{~d} w_{2}(T M)+b \mathrm{~d} B+B \frac{1}{2} \mathrm{~d} B+K_{1}{ }^{\prime} w_{1}(T M)^{2} \frac{1}{2} \mathrm{~d} B+w_{2}(T M) \frac{1}{2} \mathrm{~d} B\right) .
\end{aligned}
$$

We evaluate the path integral below:

(1) Integrating out $c$, we get

$$
\begin{aligned}
K_{1}^{\prime} \mathrm{d} w_{1}(T M) & =K_{1}^{\prime}\left(k_{(\mathbf{i})} \delta^{\perp}\left(\Sigma_{X_{(\mathbf{i})}}^{3}\right)+k_{(\mathbf{i i})} \delta^{\perp}\left(\Sigma_{X_{(i \mathbf{i})}}^{3}\right),\right. \\
K_{1}^{\prime} w_{1}(T M) & =K_{1}^{\prime}\left(k_{(\mathbf{i})} \delta^{\perp}\left(V_{X_{(\mathbf{i})}}^{4}\right)+k_{(\mathbf{i i})} \delta^{\perp}\left(V_{X_{(\mathbf{i})}}^{4}\right)\right) .
\end{aligned}
$$

We keep $K_{1}^{\prime}$ on both sides because when $K_{1}^{\prime}=1 \bmod 2$ we have this constraint; while when $K_{1}^{\prime}=0 \bmod 2$ the constraint is trivial. Using the above constraints, the path integral boils down to

$$
\begin{aligned}
\left\langle X_{(\mathbf{i})} X_{(\mathbf{i i})} U^{\prime} U_{(\mathbf{i})} U_{(\mathbf{i i})}\right\rangle= & \int[\mathcal{D} B][\mathcal{D} b][\mathcal{D} h] U^{\prime} U_{(\mathbf{i})} U_{(\mathbf{i i})} \exp \left(\mathrm{i} \pi \int_{M^{5}} h \mathrm{~d} w_{2}(T M)+b \mathrm{~d} B+B \frac{1}{2} \mathrm{~d} B\right. \\
& \left.+K_{1}{ }^{\prime} w_{1}(T M)^{2} \frac{1}{2} \mathrm{~d} B+w_{2}(T M) \frac{1}{2} \mathrm{~d} B\right)\left.\right|_{K_{1}^{\prime} w_{1}(T M)=K_{1}^{\prime}\left(k_{(\mathbf{i})} \delta^{\perp}\left(V_{X_{(i)}}^{4}\right)+k_{(i \mathbf{i})} \delta^{\perp}\left(V_{X_{(i i)}}^{4}\right)\right)} .
\end{aligned}
$$

(2) Integrating out $h$, we get

$$
\begin{aligned}
\mathrm{d} w_{2}(T M) & =k^{\prime} \delta^{\perp}\left(\Sigma_{U^{\prime}}^{2}\right), \\
w_{2}(T M) & =k^{\prime} \delta^{\perp}\left(V_{U^{\prime}}^{3}\right) .
\end{aligned}
$$


Substituting these into the path integral, we find

$$
\begin{aligned}
& \left\langle X_{(\mathbf{i})} X_{(\mathbf{i i})} U^{\prime} U_{(\mathbf{i})} U_{(\mathbf{i i})}\right\rangle=\int[\mathcal{D} B][\mathcal{D} b] U_{(\mathbf{i})} U_{(\mathbf{i i})} \exp \left(\mathrm{i} \pi \int_{M^{5}} b \mathrm{~d} B+B \frac{1}{2} \mathrm{~d} B\right. \\
& \left.+K_{1}^{\prime} w_{1}(T M)^{2} \frac{1}{2} \mathrm{~d} B\right)\left.\right|_{\substack{\left.\left.K_{1}^{\prime} w_{1}(T M)=K_{1}^{\prime}\left(k_{(\mathbf{i}}\right)^{\perp}\left(V_{X_{(i)}}^{4}\right)+k_{(i \mathbf{i})}\right)^{\perp}\left(V_{X_{(i i)}(T M)=k^{\prime} \delta^{\perp}\left(V^{3}\right.}^{3}\right)\right) \\
U_{U^{\prime}}}} .
\end{aligned}
$$

(3) Integrating out $b$, we get the constraint

$$
\begin{aligned}
\mathrm{d} B & =\ell_{(\mathbf{i})} \delta^{\perp}\left(\Sigma_{U_{(\mathbf{i})}}^{2}\right)+\ell_{(\mathbf{i i})} \delta^{\perp}\left(\Sigma_{U_{(\mathbf{i i})}}^{2}\right), \\
B & =\ell_{(\mathbf{i})} \delta^{\perp}\left(V_{U_{(\mathbf{i})}}^{3}\right)+\ell_{(\mathbf{i i})} \delta^{\perp}\left(V_{U_{(\mathbf{i})}}^{3}\right) .
\end{aligned}
$$

(4) We finally integrate out $B$ in Eq. (5.89):

$$
\begin{aligned}
& \left\langle X_{(\mathbf{i})} X_{(\mathbf{i i})} U^{\prime} U_{(\mathbf{i})} U_{(\mathbf{i i})}\right\rangle \\
& =\int[\mathcal{D B}] \exp \left(-\mathrm{i} \pi\left(\int_{M^{5}} \frac{1}{2}\left(K_{1}^{\prime} w_{1}(T M)^{2}+w_{2}(T M)\right)\left(\ell_{(\mathbf{i})} \delta^{\perp}\left(\Sigma_{U_{(i)}}^{2}\right)+\ell_{(\mathbf{i i})} \delta^{\perp}\left(\Sigma_{U_{(i \mathbf{i})}}^{2}\right)\right)\right.\right.
\end{aligned}
$$

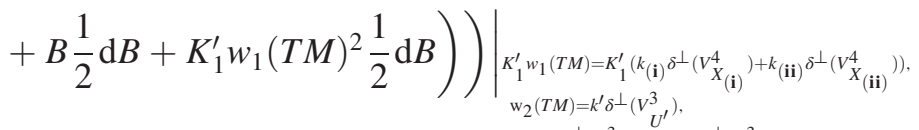

$$
\begin{aligned}
& B=\ell_{(\mathbf{i})} \delta^{\perp}\left(V_{U(\mathbf{i})}^{3}{ }^{\prime}\right)_{(i i)} \delta^{\perp}\left(V_{U}^{3}{ }_{(i i)}\right) \\
& =\int[\mathcal{D} B] \exp \left(-\mathrm{i} \pi\left(\int_{M^{5}} \frac{1}{2}\left(K_{1}^{\prime} w_{1}(T M)^{2}+w_{2}(T M)\right) \mathrm{d} B+B \frac{1}{2} \mathrm{~d} B\right.\right.
\end{aligned}
$$

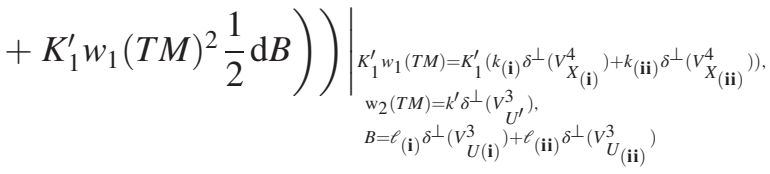

$$
\begin{aligned}
& =\exp \left(-\mathrm{i} \pi\left(K_{1}^{\prime} k_{(\mathbf{i})} k_{(\mathbf{i i})} \cdot 2 \#\left(V_{X_{(\mathbf{i})}}^{4} \cap V_{X_{(\mathbf{i})}}^{4} \cap\left(\ell_{(\mathbf{i})} \delta^{\perp}\left(\Sigma_{U_{(\mathbf{i})}}^{2}\right)+\ell_{(\mathbf{i i})} \delta^{\perp}\left(\Sigma_{U_{(\mathbf{i})}}^{2}\right)\right)\right)\right.\right. \\
& \left.\left.+\left(\frac{k^{\prime} \ell_{(\mathbf{i})}}{2} \cdot \#\left(V_{U^{\prime}}^{3} \cap \Sigma_{U_{(\mathbf{i})}}^{2}\right)+\frac{k^{\prime} \ell_{(\mathbf{i i})}}{2} \cdot \#\left(V_{U^{\prime}}^{3} \cap \Sigma_{U_{(i \mathbf{i})}}^{2}\right)\right)+\frac{\ell_{(\mathbf{i})} \ell_{(\mathbf{i i})}}{2} \cdot\left(\#\left(V_{U_{(\mathbf{i})}}^{3} \cap \Sigma_{U_{(\mathbf{i})}}^{2}\right)+\#\left(V_{U_{(i \mathbf{i})}}^{3} \cap \Sigma_{U_{(\mathbf{i})}}^{2}\right)\right)\right)\right) \\
& \cdot(\cdots) \cdot(\text { self-intersecting \# terms }) \\
& \cong \exp \left(-\mathrm{i} \pi\left(K_{1}^{\prime}\left(k_{(\mathbf{i})} k_{(\mathbf{i i})} \ell_{(\mathbf{i})} \cdot 2 \mathrm{Tlk}^{(5)}\left(\Sigma_{X_{(\mathbf{i})}}^{3}, \Sigma_{X_{(\mathbf{i i})}}^{3}, \Sigma_{U_{(\mathbf{i})}}^{2}\right)+k_{(\mathbf{i})} k_{(\mathbf{i i})} \ell_{(\mathbf{i i})} \cdot 2 \operatorname{Tlk}^{(5)}\left(\Sigma_{X_{(\mathbf{i})}}^{3}, \Sigma_{X_{(\mathbf{i})}}^{3}, \Sigma_{U_{(\mathbf{i})}}^{2}\right)\right)\right.\right. \\
& \left.\left.+\left(\frac{k^{\prime} \ell_{(\mathbf{i})}}{2} \cdot \mathrm{Lk}^{(5)}\left(\Sigma_{U^{\prime}}^{2}, \Sigma_{U_{(\mathbf{i})}}^{2}\right)+\frac{k^{\prime} \ell_{(\mathbf{i i})}}{2} \cdot \mathrm{Lk}^{(5)}\left(\Sigma_{U^{\prime}}^{2}, \Sigma_{U_{(\mathbf{i})}}^{2}\right)\right)+\ell_{(\mathbf{i})} \ell_{(\mathbf{i i})} \cdot \mathrm{Lk}^{(5)}\left(\Sigma_{U_{(\mathbf{i})}}^{2}, \Sigma_{U_{(\mathbf{i})}}^{2}\right)\right)\right) \cdot(\cdots) .
\end{aligned}
$$

We propose a setup to remove or renormalize the (self-intersecting \# terms) that appeared in Eq. (5.94), following the same strategy as footnote 40 .

For $\mathbf{S}=\pi \int_{M^{5}} K_{1}^{\prime} c \mathrm{~d} w_{1}(T M)+h \mathrm{~d} w_{2}(T M)+b \mathrm{~d} B+B \frac{1}{2} \mathrm{~d} B+K_{1}^{\prime} w_{1}(T M)^{2} \frac{1}{2} \mathrm{~d} B+w_{2}(T M) \frac{1}{2} \mathrm{~d} B$, we derive the link invariant for the $5 \mathrm{~d}$ TQFT $\mathbf{Z}_{\mathrm{SET}}\left[M^{5}\right]$ in Eq. (5.94) and Eq. (5.95):

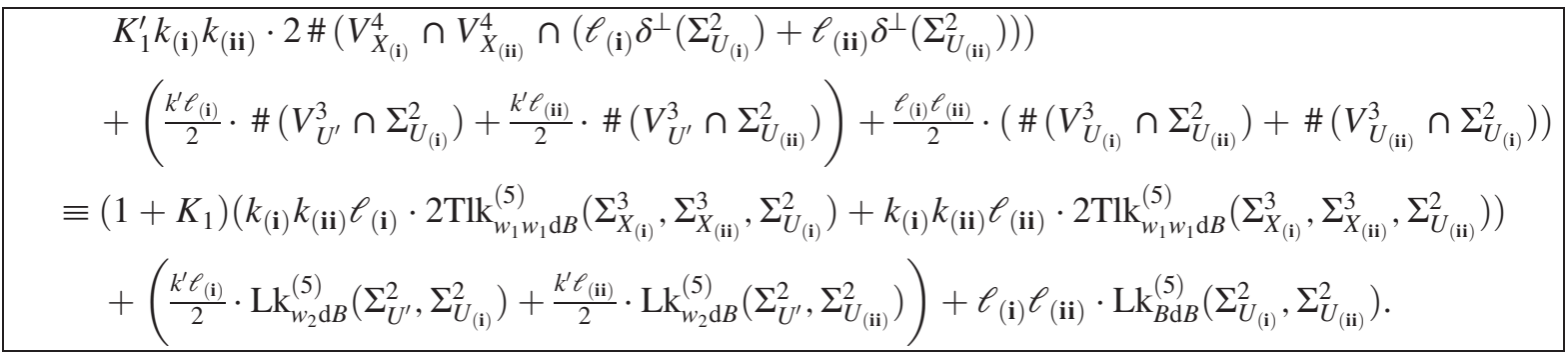


The path integral with appropriate extended operator insertions becomes Eq. (5.95) which provides the above link invariant.

\section{3. $\left(K_{1}, K_{2}\right)=(0,0)$ or $(0,1)$ : First and third siblings}

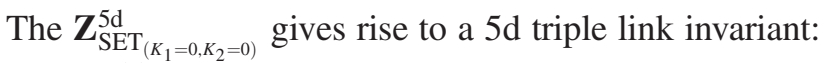

(i) $\mathrm{Tlk}_{w_{1} B B}^{(5)}$ in Eq. (5.25) of Sec. VA. We present an exemplary link configuration later in Sec. VI B that can be detected by this link invariant.

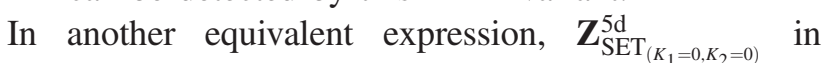
Eq. (4.5) gives rise to other link invariants in Eq. (5.96) including

(i) $\operatorname{Tlk}_{w_{1} w_{1} \mathrm{~d} B}^{(5)}\left(\Sigma_{X_{(\mathbf{i})}}^{3}, \Sigma_{X_{(\mathbf{i i})}}^{3}, \Sigma_{U}^{2}\right)$, a second type of triple link in $5 \mathrm{~d}$ (although seemly undetectable due to an exponent factor $2 \pi$ in the expectation value). We present an exemplary link configuration later in (Sec. VI C) that can be detected by this link invariant.

(ii) $\operatorname{Lk}_{B \mathrm{~d} B}^{(5)}\left(\Sigma_{U_{(\mathrm{i})}}^{2}, \Sigma_{U_{(i \mathrm{i})}}^{2}\right)$, a quadratic link of two-surfaces in $5 \mathrm{~d}$. We present an exemplary link configuration later in Sec. VIE that can be detected by this link invariant.

(iii) $\mathrm{Lk}_{w_{2} \mathrm{~d} B}^{(5)}\left(\Sigma_{U^{\prime}}^{2}, \Sigma_{U}^{2}\right)$, another quadratic link of twosurfaces in $5 \mathrm{~d}$. We present an exemplary link configuration later in Sec. VIF that can be detected by this link invariant.

Physically, these link invariants may be related to each other by rearranging the spacetime braiding process of strings/branes. It will be interesting to find a precise mathematical equality to relate these link invariants.

\section{4. $\left(K_{1}, K_{2}\right)=(1,0)$ or $(1,1):$ Second and fourth siblings}

$\mathbf{Z}_{\mathrm{SET}_{\left(K_{1}=1, K_{2}=0\right)}^{\mathrm{d}}}^{\mathrm{d}}$ in Eq. (4.5) gives rise to link invariants in Eq. (5.96) including the following:

(i) $\operatorname{Lk}_{B \mathrm{~d} B}^{(5)}\left(\Sigma_{U_{(\mathrm{i})}}^{2}, \Sigma_{U_{(i \mathrm{i})}}^{2}\right)$, a quadratic link of two-surfaces in $5 \mathrm{~d}$. We present an exemplary link configuration later in (Sec. VIE) that can be detected by this link invariant.

(ii) $\mathrm{Lk}_{w_{2} \mathrm{~d} B}^{(5)}\left(\Sigma_{U^{\prime}}^{2}, \Sigma_{U}^{2}\right)$, another quadratic link of twosurfaces in $5 \mathrm{~d}$. We present an exemplary link configuration later in Sec. VIF that can be detected by this link invariant.

Similar to our comments above in Sec. V D 3, it will be interesting to find a precise mathematical equality to relate these link invariants.

\section{ANYONIC STRING/BRANE SPACETIME BRAIDING PROCESSES AND LINK CONFIGURATIONS OF EXTENDED OPERATORS}

We provide the exemplary spacetime braiding processes of anyonic strings and branes in general dimensions (with an emphasis on 5d) and the link configurations of extended operators, which can be detected by the link invariants that we derived in Sec. V.

\section{A. Quadratic link (Aharanov-Bohm) configuration in any dimension}

To warm up, we first discuss the quadratic link, associated with the Aharanov-Bohm statistics in $d$ d due to the linking of one world line of the charged particle and the $(d-2) d$ world sheet of the fractional flux. In $3 d$ spacetime, we have the following presentation:

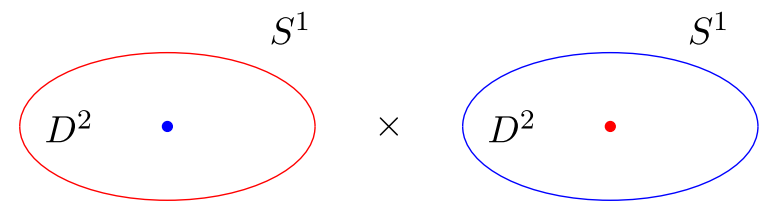

where gluing two solid tori $D^{2} \times S^{1}$ gives rise to a threesphere: $\left(D_{\mathrm{L}}^{2} \times S_{\mathrm{R}}^{1}\right) \cup\left(S_{\mathrm{L}}^{1} \times D_{\mathrm{R}}^{2}\right)=S^{3}$. We represent the two solid tori as a blue solid tori and a red solid tori, $\left(D_{\mathrm{L}}^{2} \times S_{\mathrm{R}}^{1}\right) \cup\left(S_{\mathrm{L}}^{1} \times D_{\mathrm{R}}^{2}\right)=S^{3}$. The quadratic link invariant detecting this Aharanov-Bohm configuration is given by Ref. [12] and references therein: $\operatorname{Lk}\left(\left(0_{\mathrm{pt}}\right)_{\mathrm{L}} \times S_{\mathrm{R}}^{1}, S_{\mathrm{L}}^{1} \times\right.$ $\left.\left(0_{\mathrm{pt}}\right)_{\mathrm{R}}\right)$, which we also express as

$$
\operatorname{Lk}\left(\left(0_{\mathrm{pt}}\right)_{\mathrm{L}} \times S_{\mathrm{R}}^{1}, S_{\mathrm{L}}^{1} \times\left(0_{\mathrm{pt}}\right)_{\mathrm{R}}\right)
$$

based on the color labeling of the inclusion of two $S^{1}$ circles belonging to which of the two solid tori. This link invariant can be computed from the intersection number,

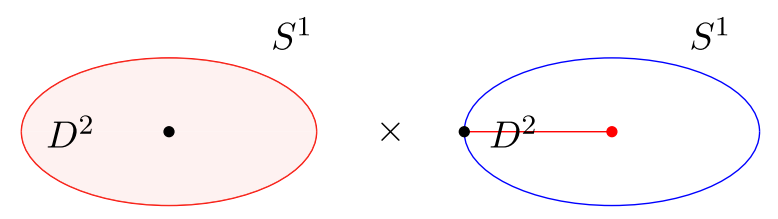

where

$$
\left.\#\left(\left(0_{\mathrm{pt}}\right)_{\mathrm{L}} \times S_{\mathrm{R}}^{1}\right) \cap\left(D_{\mathrm{L}}^{2} \times\left(0_{\mathrm{pt}-}\right)_{\mathrm{R}}\right)\right)=1 .
$$

$\left(0_{\mathrm{pt}-}\right)$ means the point $\left(0_{\mathrm{pt}}\right)$ now is attached to a line. The intersection number $\left.\#\left(\left(0_{\mathrm{pt}}\right)_{\mathrm{L}} \times S_{\mathrm{R}}^{1}\right) \cap\left(D_{\mathrm{L}}^{2} \times\left(0_{\mathrm{pt}-}\right)_{\mathrm{R}}\right)\right)=1$ precisely corresponds to the black dot $\bullet$.

In $d \mathrm{~d}$ spacetime, $S^{d}$ can be obtained by

$$
\left(D_{\mathrm{L}}^{d-1} \times S_{\mathrm{R}}^{1}\right) \cup\left(S_{\mathrm{L}}^{d-2} \times D_{\mathrm{R}}^{2}\right)=S^{d},
$$

which can be graphically represented as 


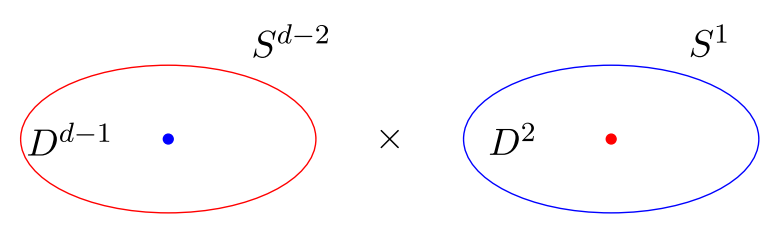

The associated link invariant is

$$
\operatorname{Lk}\left(\left(0_{\mathrm{pt}}\right)_{\mathrm{L}} \times S_{\mathrm{R}}^{1}, S_{\mathrm{L}}^{d-2} \times\left(0_{\mathrm{pt}}\right)_{\mathrm{R}}\right)
$$

with the color prescription explained earlier. This link invariant can be computed from the intersection number,

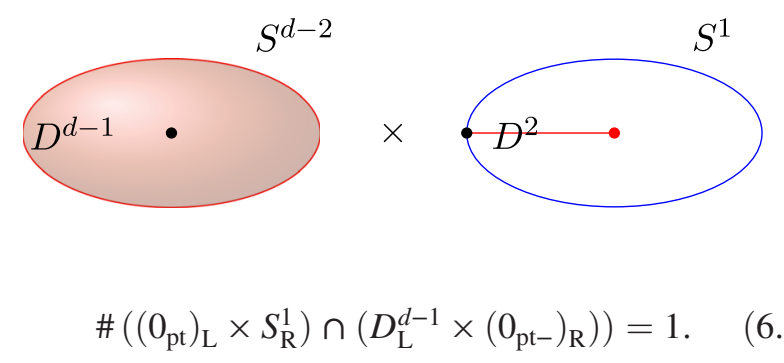

Here, $\left(0_{\mathrm{pt}-}\right)$ means the point $\left(0_{\mathrm{pt}}\right)$ now is attached with a line. We see the intersection number $\#\left(\left(0_{\mathrm{pt}}\right)_{\mathrm{L}} \times S_{\mathrm{R}}^{1}\right) \cap$ $\left.\left(D_{\mathrm{L}}^{d-1} \times\left(0_{\mathrm{pt}-}\right)_{\mathrm{R}}\right)\right)=1$ precisely corresponds to the black dot $\bullet$.

B. The first triple link \# $\left(V_{X}^{4} \cap V_{U_{(i)}}^{3} \cap V_{U_{(i)}}^{3}\right) \equiv$ $\mathbf{T l k}_{w_{1} B B}^{(5)}\left(\Sigma_{X}^{3}, \Sigma_{U_{(\mathrm{i})}}^{2}, \Sigma_{U_{(i \mathrm{i})}}^{2}\right)$ configuration in 5d

We proceed to discuss the triple link configuration for $\mathrm{Tlk}_{w_{1} B B}^{(5)}\left(\Sigma_{X}^{3}, \Sigma_{U_{(i)}}^{2}, \Sigma_{U_{(i \mathrm{i})}}^{2}\right)$ derived in Sec. VA. ${ }^{43}$ We propose that this link invariant derived in Sec. VA can detect the link configuration in Fig. 2.

To explain, we start by constructing the five-sphere via $S^{5}=\partial D^{6}=\partial\left(D^{4} \times D^{2}\right)=S^{3} \times D^{2} \cup D^{4} \times S^{1}=S^{3} \times D^{2} \cup$ $D^{2} \times D^{2} \times S^{1}$. More explicitly, we color the different components as $S^{5}=\left(S_{\mathrm{L}}^{3} \times D_{\mathrm{R}}^{2}\right) \cup\left(D_{\mathrm{L}}^{4} \times S_{\mathrm{R}}^{1}\right)$ and $S^{5}=\left(S_{\mathrm{L}}^{3} \times\right.$ $\left.D_{\mathrm{R}}^{2}\right) \cup\left(D_{\mathrm{L}}^{2} \times D_{\mathrm{L}}^{2} \times S_{\mathrm{R}}^{1}\right)$.

Consider the link invariant defined by \# $\left(V_{X}^{4} \cap V_{U_{(\mathrm{i})}}^{3} \cap\right.$ $\left.V_{U_{(i i)}}^{3}\right) \equiv \operatorname{Tlk}_{w_{1} B B}^{(5)}\left(\Sigma_{X}^{3}, \Sigma_{U_{(i)}}^{2}, \Sigma_{U_{(i i)}}^{2}\right)$, where we see that the link configuration in Fig. 2 gives the intersection number 1 in Fig. 3. Again in Fig. 3 associated with the intersection number $\#\left(V_{X}^{4} \cap V_{U_{(i)}}^{3} \cap V_{U_{(i i)}}^{3}\right),\left(0_{\mathrm{pt}-}\right)$ means the point $\left(0_{\mathrm{pt}}\right)$ now is attached to a line. We see the intersection number \# $\left(V_{X}^{4} \cap V_{U_{(i)}}^{3} \cap V_{U_{(i i)}}^{3}\right)=1$ precisely corresponds to the black dot $\bullet$.

\footnotetext{
${ }^{43}$ Effectively, $\operatorname{Tlk}_{w_{1} B B}^{(5)}\left(\Sigma_{X}^{3}, \Sigma_{U_{(\mathrm{i})}}^{2}, \Sigma_{U_{(\mathrm{ii})}}^{2}\right)$ can also be regarded as $\mathrm{Tlk}_{A B B}^{(5)}\left(\Sigma_{X}^{3}, \Sigma_{U_{(\mathrm{i})}}^{2}, \Sigma_{U_{(i \mathrm{i})}}^{2}\right)$ where $A$ is the other $\mathbb{Z}_{n}$ one-form gauge field.
}

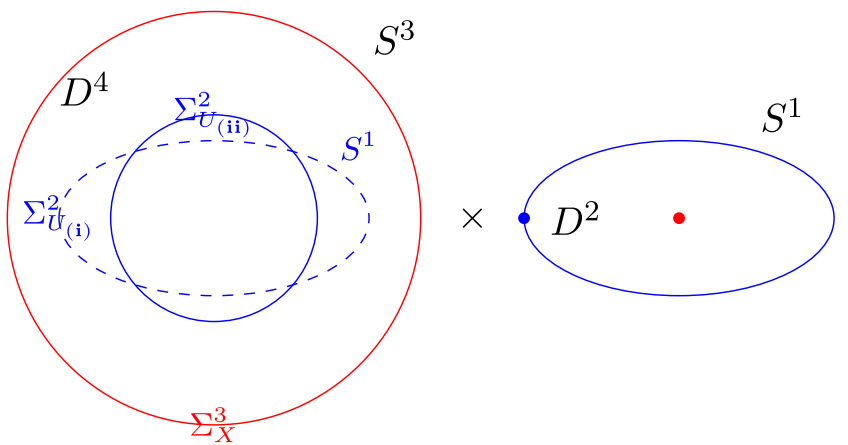

FIG. 2. $S^{5}=\partial D^{6}=\partial\left(D^{4} \times D^{2}\right)=S^{3} \times D^{2} \cup D^{4} \times S^{1}=S^{3} \times$ $D^{2} \cup D^{2} \times D^{2} \times S^{1}$, the intersection of the two copies of $D^{2} \times S^{1}$ in the second piece $\left(D^{2} \times 0_{\mathrm{pt}} \times S^{1}\right.$ and $\left.0_{\mathrm{pt}} \times D^{2} \times S^{1}\right)$ is $0_{\mathrm{pt}} \times 0_{\mathrm{pt}} \times S^{1}=0_{\mathrm{pt}} \times S^{1}$; this $0_{\mathrm{pt}} \times S^{1}$ and $S^{3} \times 0_{\mathrm{pt}}$ in the first piece are linked. In this figure, $\Sigma_{X}^{3}=S^{3} \times 0_{\mathrm{pt}}, \quad \Sigma_{U_{(i)}}^{2}=$ $\partial\left(D^{2} \times 0_{\mathrm{pt}} \times S^{1}\right), \Sigma_{U_{(i i)}}^{2}=\partial\left(0_{\mathrm{pt}} \times D^{2} \times S^{1}\right)$.

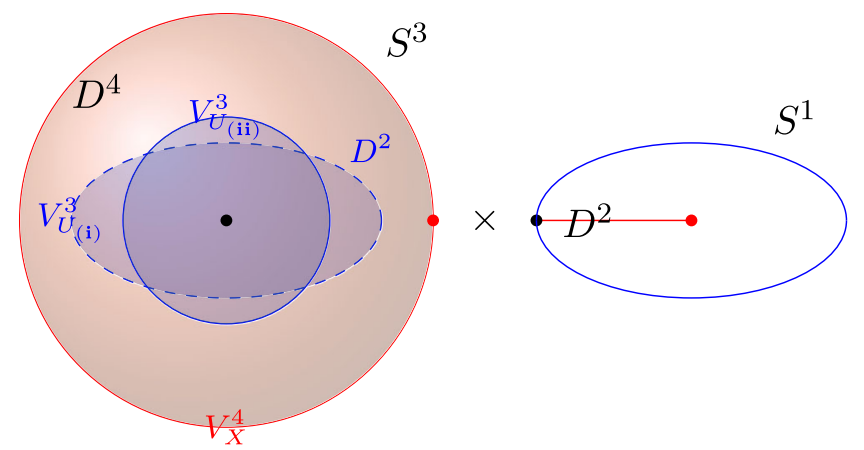

FIG. 3. Following Fig. $2, V_{X}^{4}=D^{4} \times 0_{\mathrm{pt}}$ which bounds $\Sigma_{X}^{3}$, $V_{U_{(i)}}^{3}=D^{2} \times 0_{\mathrm{pt}} \times S^{1} \quad$ which bounds $\quad \Sigma_{U_{(\mathrm{i})}}^{2}, \quad V_{U_{(\mathrm{i})}}^{3}=0_{\mathrm{pt}} \times$ $D^{2} \times S^{1}$ which bounds $\Sigma_{U_{(i \mathrm{i})}}^{2}$. The intersection of $V_{U_{(\mathrm{i})}}^{3}$ and $V_{U_{(i i)}}^{3}$ is $0_{\mathrm{pt}} \times S^{1}$, the intersection of $V_{X}^{4}$ and this $0_{\mathrm{pt}} \times S^{1}$ is a point which is the point in black in this figure.

\section{The second triple link \# $\left(V_{X_{(i)}}^{4} \cap V_{X_{(i i)}}^{4} \cap \Sigma_{U}^{2}\right) \equiv$ $\mathbf{T I k}_{w_{1} w_{1} \mathrm{~d} B}^{(5)}\left(\Sigma_{X_{(\mathrm{i})}}^{3}, \Sigma_{X_{(i \mathrm{i})}}^{3}, \Sigma_{U}^{2}\right)$ configuration in $\mathbf{5 d}$}

We now discuss $\operatorname{Tlk}_{w_{1} w_{1} \mathrm{~d} B}^{(5)}\left(\Sigma_{X_{(\mathrm{i})}}^{3}, \Sigma_{X_{(i \mathrm{i})}}^{3}, \Sigma_{U}^{2}\right)$, or schematically $\operatorname{Tlk}_{A A \mathrm{~d} B}^{(5)}\left(\Sigma_{X_{(\mathrm{i})}}^{3}, \Sigma_{X_{(i \mathrm{i})}}^{3}, \Sigma_{U}^{2}\right)$. This link invariant is derived in Sec. V B 2.

Let us consider the link invariant defined by $\#\left(V_{X_{(i)}}^{4} \cap\right.$ $\left.V_{X_{(i i)}}^{4} \cap \Sigma_{U}^{2}\right) \equiv \operatorname{Tlk}_{w_{1} w_{1} \mathrm{~d} B}^{(5)}\left(\Sigma_{X_{(i)}}^{3}, \Sigma_{X_{(i i)}}^{3}, \Sigma_{U}^{2}\right)$. We see that the link configuration in Fig. 4 gives the intersection number 1 in Fig. 5.

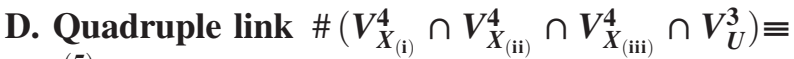

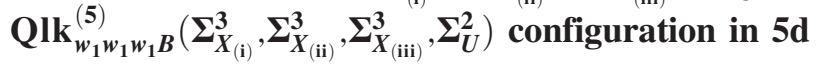

We now discuss Q $\mathrm{lk}_{w_{1} w_{1} w_{1} B}^{(5)}\left(\Sigma_{X_{(\mathrm{i})}}^{3}, \Sigma_{X_{(\mathrm{ii})}}^{3}, \Sigma_{X_{(\mathrm{iii})}}^{3}\right)$, or schematically Qlk $\mathrm{Qaab}_{(\mathrm{a})}^{(5)}\left(\Sigma_{X_{(\mathrm{i})}}^{3}, \Sigma_{X_{(\mathrm{ii})}}^{3}, \Sigma_{X_{(\mathrm{iii})}}^{3}\right)$. This link invariant is derived in Sec. V B 1. 


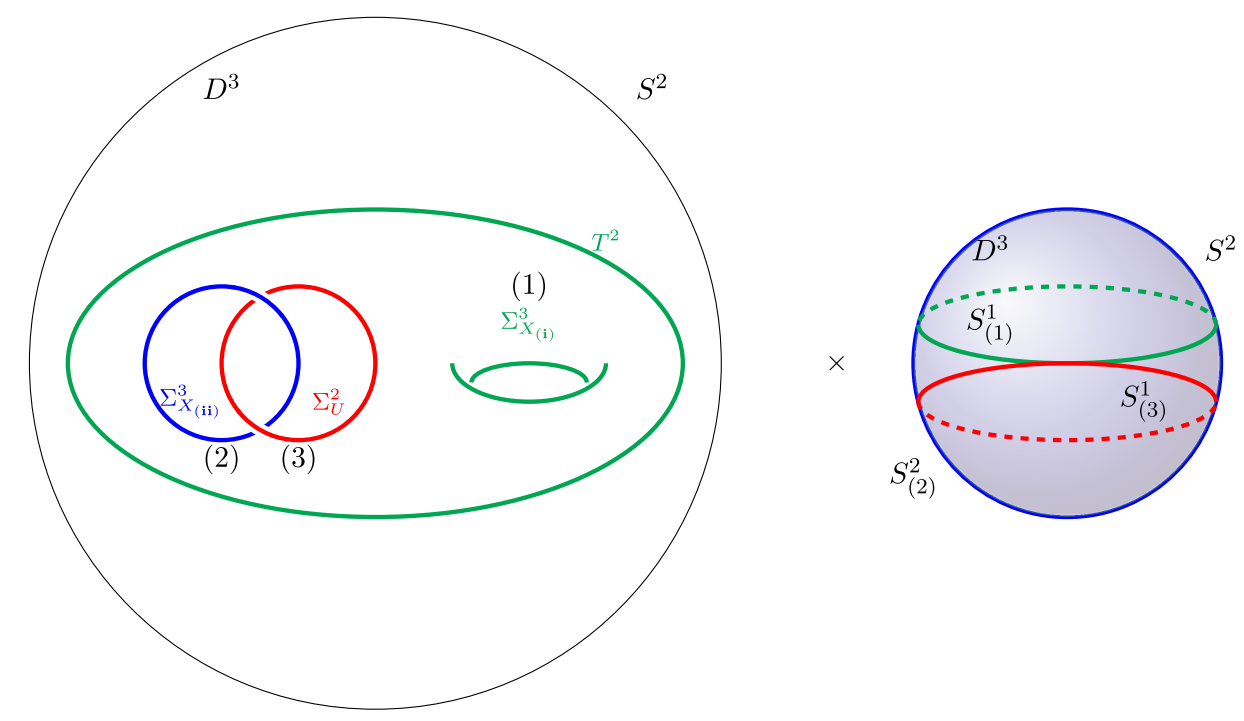

FIG. 4. $\quad S^{5}=\partial D^{6}=\partial\left(D^{3} \times D^{3}\right)=S^{2} \times D^{3} \cup D^{3} \times S^{2}$. Put a two-torus [denoted by (1)] in $D^{3} \times 0_{\mathrm{pt}}$, and put a Hopf link [the two circles are denoted by (2) and (3), respectively] in the solid two-torus. Put two circles [denoted by $S_{(1)}^{1}$ and $S_{(3)}^{1}$, respectively] which intersect in only one point in $0_{\mathrm{pt}} \times S^{2}$ (denoted by $S_{(2)}^{2}$ ). In this figure, $\Sigma_{X_{(\mathrm{i})}}^{3}$ is the Cartesian product of the two-torus (1) and $S_{(1)}^{1}$, $\Sigma_{X_{(i \mathrm{i})}}^{3}$ is the Cartesian product of the circle (2) and $S_{(2)}^{2}, \Sigma_{U}^{2}$ is the Cartesian product of the circle (3) and $S_{(3)}^{1}$.

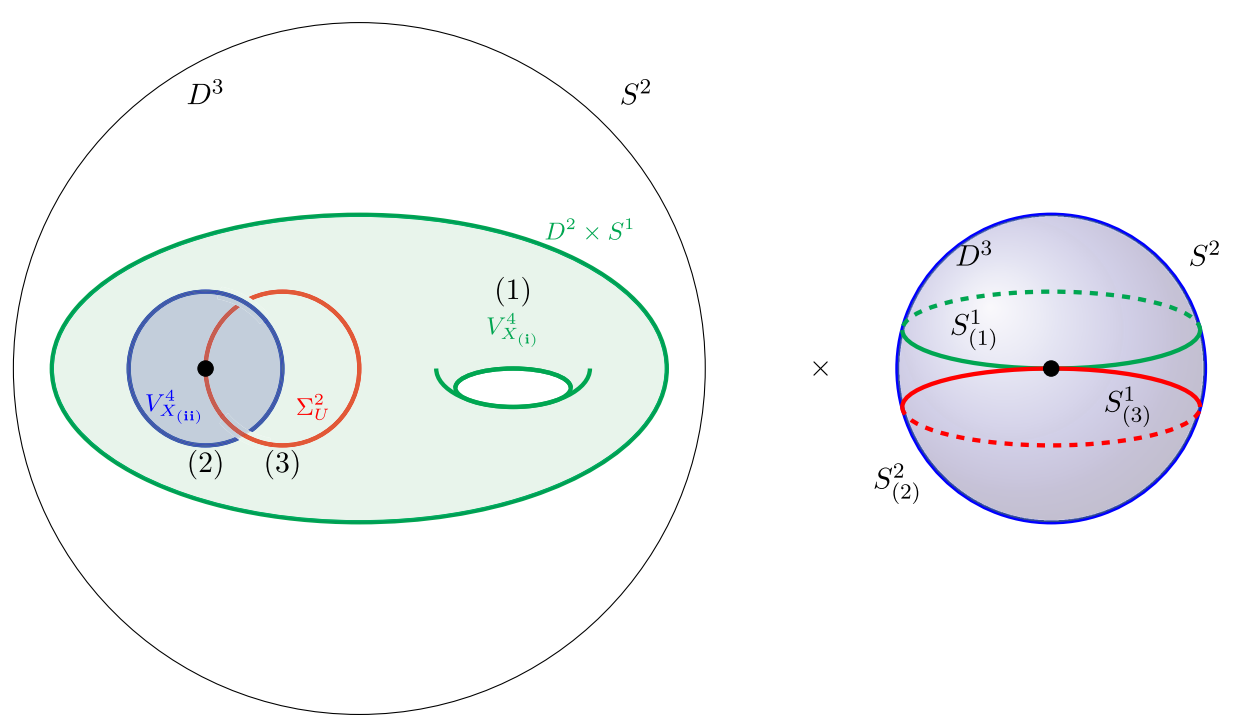

FIG. 5. Following Fig. 4, if we fill in $\Sigma_{X_{(i)}}^{3}$ and $\Sigma_{X_{(i i)}}^{3}$, we get $V_{X_{(i)}}^{4}=D^{2} \times S^{1} \times S^{1}$ and $V_{X_{(i i)}}^{4}=D^{2} \times S^{2} . V_{X_{(i)}}^{4}, V_{X_{(i i)}}^{4}$, and $\Sigma_{U}^{2}$ will intersect in only one point which is the point in black in this figure.

Let us consider the link invariant defined by

$$
\begin{aligned}
& \#\left(V_{X_{(\mathrm{i})}}^{4} \cap V_{X_{(\mathrm{ii})}}^{4} \cap V_{X_{(\mathrm{iii})}}^{4} \cap V_{U}^{3}\right) \\
& \quad \equiv \mathrm{Qlk}_{w_{1} w_{1} w_{1} B}^{(5)}\left(\Sigma_{X_{(\mathrm{i})}}^{3}, \Sigma_{X_{(\mathrm{ii})}}^{3}, \Sigma_{X_{(\mathrm{iii})}}^{3}, \Sigma_{U}^{2}\right) .
\end{aligned}
$$

We see that the link configuration in Fig. 6 gives the intersection number 1 in Fig. 7.
E. Quadratic link \# $\left(\boldsymbol{V}_{U_{(\mathrm{i})}}^{\mathbf{3}} \cap \boldsymbol{\Sigma}_{U_{(\mathrm{ii})}}^{2}\right) \equiv \mathbf{L} \mathbf{k}_{\boldsymbol{B d B} \boldsymbol{B}}^{(\mathbf{5})}\left(\boldsymbol{\Sigma}_{U_{(\mathrm{i})}}^{2}, \Sigma_{U_{(\mathrm{ii})}}^{2}\right)$

Now we discuss $\operatorname{Lk}_{B \mathrm{~d} B}^{(5)}\left(\Sigma_{U_{(\mathrm{i})}}^{2}, \Sigma_{U_{(i i)}}^{2}\right)$. This link invariant is derived in Sec. V D.

Let us consider the link invariant defined by

$$
\begin{aligned}
& \#\left(V_{X_{(\mathrm{i})}}^{4} \cap V_{X_{(\mathrm{ii})}}^{4} \cap V_{X_{(\mathrm{iii})}}^{4} \cap V_{U}^{3}\right) \\
& \quad \equiv \mathrm{Qk}_{w_{1} w_{1} w_{1} B}^{(5)}\left(\Sigma_{X_{(\mathrm{i})}}^{3}, \Sigma_{X_{(i \mathrm{i})}}^{3}, \Sigma_{X_{(\mathrm{iii})}}^{3}, \Sigma_{U}^{2}\right) .
\end{aligned}
$$



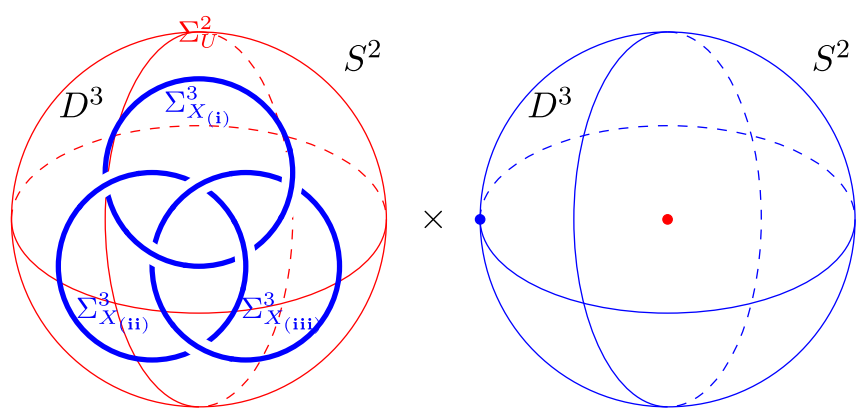

FIG. 6. $S^{5}=\partial D^{6}=\partial\left(D^{3} \times D^{3}\right)=S^{2} \times D^{3} \cup D^{3} \times S^{2}$. Put Borromean rings in $D^{3} \times 0_{\mathrm{p} t}$. If we fill in each of the three circles of the Borromean rings, then we get an intersection point, and we can think of this point as $0_{\mathrm{pt}}$ in $D^{3}$. Then the Cartesian product of each of the three circles and $S^{2}$ (denoted by $\Sigma_{X_{(i)}}^{3}, \Sigma_{X_{(i i)}}^{3}$, and $\Sigma_{X_{(i i i)}}^{3}$, respectively) will intersect in $0_{\mathrm{pt}} \times S^{2}$; this $0_{\mathrm{pt}} \times S^{2}$ and $S^{2} \times 0_{\mathrm{pt}}\left(\Sigma_{U}^{2}\right.$ in this figure) are linked.
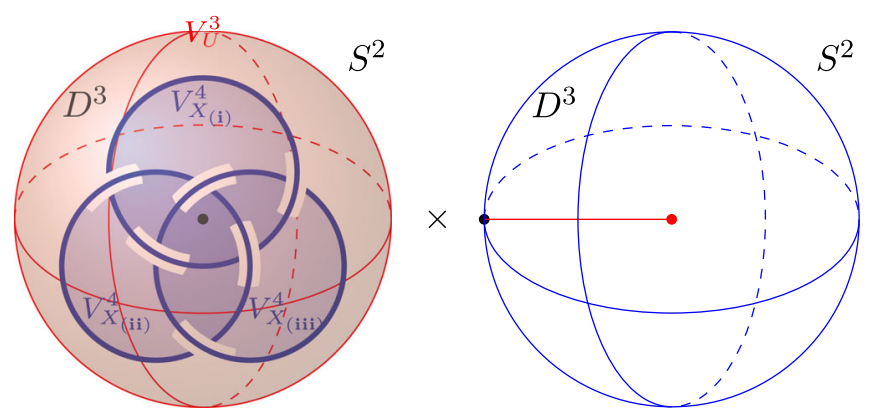

FIG. 7. Following Fig. 6, we denote the three $D^{2} \times S^{2}$ which bound the Cartesian product of the three circles and $S^{2}$ as $V_{X_{(i)}}^{4}$, $V_{X_{(i i)}}^{4}, V_{X_{(i i i)}}^{4}$, respectively. The intersection of $V_{X_{(i)}}^{4}, V_{X_{(i i)}}^{4}$, and $V_{X_{(i i i)}}^{4}$ is $0_{\mathrm{pt}} \times S^{2}$. The intersection of $V_{U}^{3}=D^{3} \times 0_{\mathrm{pt}}$ which bounds $\Sigma_{U}^{2}$ and $0_{\mathrm{pt}} \times S^{2}$ is a point which is the point in black in this figure.

We see that the link configuration in Fig. 8 gives the intersection number 1 in Fig. 9.

\section{F. Quadratic link \# $\left(\boldsymbol{V}_{U^{\prime}}^{\mathbf{3}} \cap \boldsymbol{\Sigma}_{U}^{2}\right) \equiv \mathbf{L k}_{w_{2} \mathrm{~d} B}^{(\mathbf{5})}\left(\boldsymbol{\Sigma}_{U}^{2}, \boldsymbol{\Sigma}_{U^{\prime}}^{2}\right)$}

Now we discuss $\mathrm{Lk}_{w_{2} \mathrm{~d} B}^{(5)}\left(\Sigma_{U^{\prime}}^{2}, \Sigma_{U}^{2}\right)$ or $\mathrm{Lk}_{B^{\prime} \mathrm{d} B}^{(5)}\left(\Sigma_{U^{\prime}}^{2}, \Sigma_{U}^{2}\right)$. This link invariant is derived in Sec. V C.

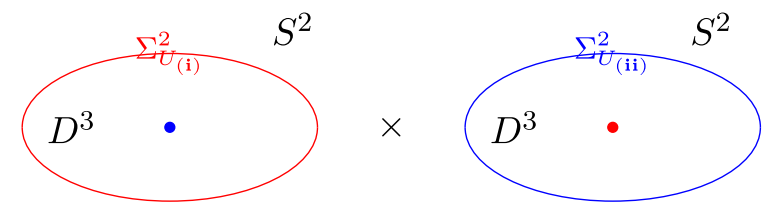

FIG. 8. $S^{5}=\partial D^{6}=\partial\left(D^{3} \times D^{3}\right)=S^{2} \times D^{3} \cup D^{3} \times S^{2}$. The $S^{2} \times 0_{\mathrm{pt}}$ in the first piece and the $0_{\mathrm{pt}} \times S^{2}$ in the second piece are linked. In this figure, $\Sigma_{U_{(\mathrm{i})}}^{2}=S^{2} \times 0_{\mathrm{pt}}$ and $\Sigma_{U_{(\mathrm{ii})}}^{2}=0_{\mathrm{pt}} \times S^{2}$.

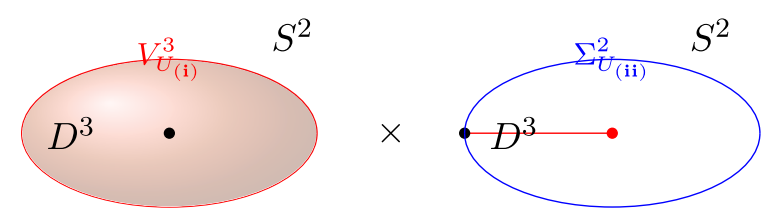

FIG. 9. Following Fig. 8, if we fill in $S^{2} \times 0_{\mathrm{pt}}$, we get $V_{U_{(\mathrm{i})}}^{3}=D^{3} \times 0_{\mathrm{pt}}$; the intersection of $D^{3} \times 0_{\mathrm{pt}}$ and $0_{\mathrm{pt}} \times S^{2}$ is a point which is the point in black in this figure.

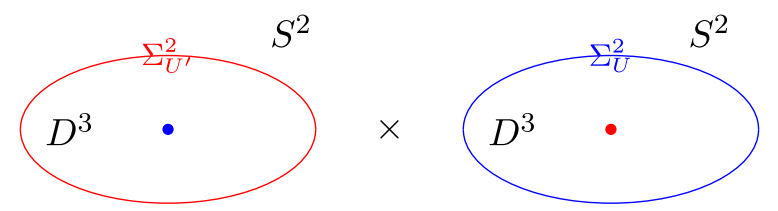

FIG. 10. $\quad S^{5}=\partial D^{6}=\partial\left(D^{3} \times D^{3}\right)=S^{2} \times D^{3} \cup D^{3} \times S^{2}$. The $S^{2} \times 0_{\mathrm{pt}}$ in the first piece and the $0_{\mathrm{pt}} \times S^{2}$ in the second piece are linked. In this figure, $\Sigma_{U^{\prime}}^{2}=S^{2} \times 0_{\mathrm{pt}}$ and $\Sigma_{U}^{2}=0_{\mathrm{pt}} \times S^{2}$.

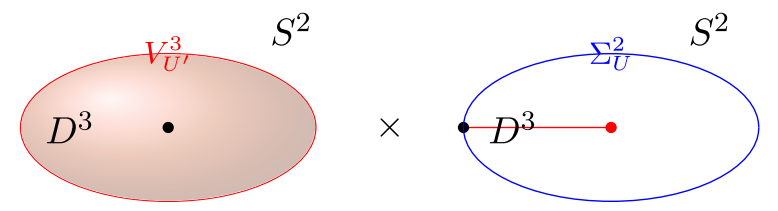

FIG. 11. Following Fig. 10, if we fill in $S^{2} \times 0_{\mathrm{pt}}$, we get $V_{U^{\prime}}^{3}=D^{3} \times 0_{\mathrm{pt}}$, the intersection of $D^{3} \times 0_{\mathrm{pt}}$ and $0_{\mathrm{pt}} \times S^{2}$ is a point which is the point in black in this figure.

Let us consider the link invariant defined by \# $\left(V_{U^{\prime}}^{3} \cap\right.$ $\left.\Sigma_{U}^{2}\right) \equiv \operatorname{Lk}_{w_{2} \mathrm{~d} B}^{(5)}\left(\Sigma_{U}^{2}, \Sigma_{U^{\prime}}^{2}\right)$. We see that the link configuration in Fig. 10 gives the intersection number 1 in Fig. 11.

G. The third triple link $\#\left(V_{X_{(i)}}^{4} \cap \Sigma_{X_{(i)}}^{3} \cap V_{U}^{3}\right) \equiv$ $\operatorname{TIk}_{(A \mathrm{~d} A) B}^{(5)}\left(\Sigma_{X_{(\mathrm{i})}}^{3}, \Sigma_{X_{(i \mathrm{i})}}^{3}, \Sigma_{U}^{2}\right)$ configuration in 5d

Finally, we discuss a third triple link invariant $\#\left(V_{X_{(\mathrm{i})}}^{4} \cap \Sigma_{X_{(\mathrm{ii})}}^{3} \cap V_{U}^{3}\right) \equiv \operatorname{Tlk}_{(A \mathrm{~d} A) B}^{(5)}\left(\Sigma_{X_{(\mathrm{i})}}^{3}, \Sigma_{X_{(\mathrm{ii})}}^{3}, \Sigma_{U}^{2}\right)$. We have not derived these from $4 \mathrm{~d}$ YM-5d SET coupled systems.

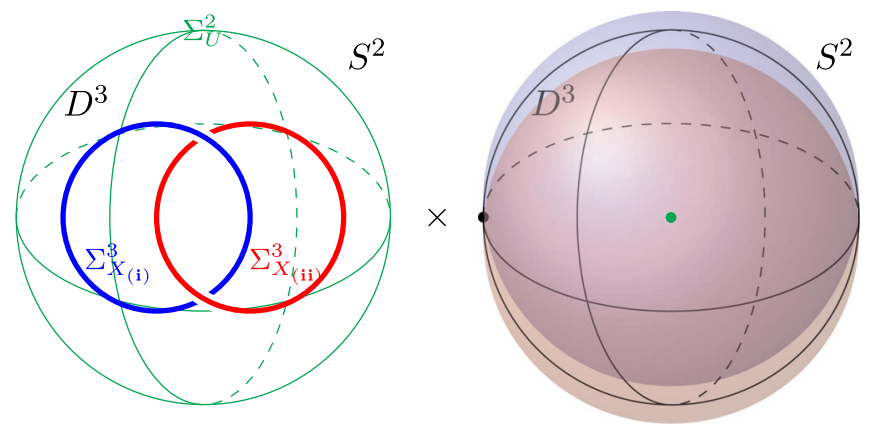

FIG. 12. $\quad S^{5}=\partial D^{6}=\partial\left(D^{3} \times D^{3}\right)=S^{2} \times D^{3} \cup D^{3} \times S^{2}$, and put a Hopf link in $D^{3} \times 0_{\mathrm{pt}}$. In this figure, $\Sigma_{X_{(\mathrm{i})}}^{3}$ and $\Sigma_{X_{(\mathrm{i})}}^{3}$ are the Cartesian product of the two circles in the Hopf link and $S^{2}$, respectively; namely, they are both $S^{1} \times S^{2}$ and $\Sigma_{U}^{2}=S^{2} \times 0_{\mathrm{pt}}$. 

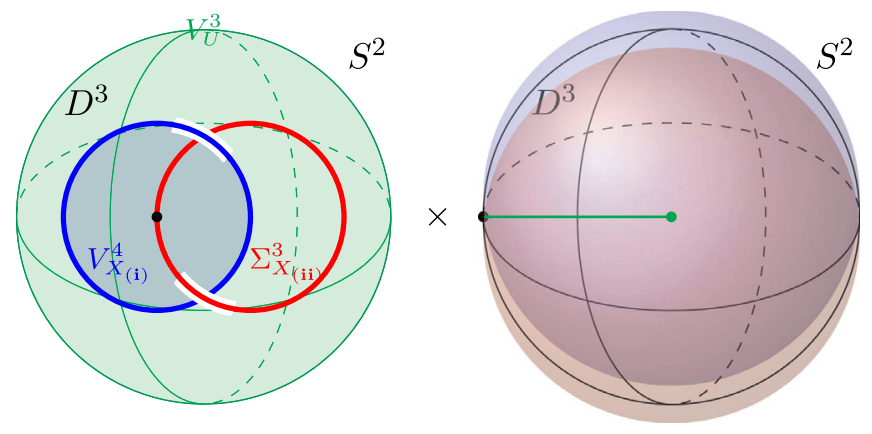

FIG. 13. Following Fig. 12, if we fill in $\Sigma_{X_{(\mathrm{i})}}^{3}$, we get $V_{X_{(i)}}^{4}=D^{2} \times S^{2}$; the intersection of $V_{X_{(i)}}^{4}$ and $\Sigma_{X_{(i \mathbf{i})}}^{3}$ is the Cartesian product of a point (we can think of the point as $0_{\mathrm{pt}}$ ) and $S^{2}$. If we fill in $\Sigma_{U}^{2}$ further, we get $V_{U}^{3}=D^{3} \times 0_{\mathrm{pt}}$; the intersection of $D^{3} \times 0_{\mathrm{pt}}$ and $0_{\mathrm{pt}} \times S^{2}$ is a point which is the point in black in this figure.

However, to get this, we need a topological term $\left(w_{1}(T M) \mathrm{d} w_{1}(T M)\right) B$. This is possible, however, from the $\left(A_{I} \mathrm{~d} A_{J}\right) B$ type of TQFTs.

Let us consider the link invariant defined by $\#\left(V_{X_{(i)}}^{4} \cap\right.$ $\left.\Sigma_{X_{(i i)}}^{3} \cap V_{U}^{3}\right) \equiv \operatorname{Tlk}_{(A \mathrm{~d} A) B}^{(5)}\left(\Sigma_{X_{(\mathrm{i})}}^{3}, \Sigma_{X_{(\mathrm{ii})}}^{3}, \Sigma_{U}^{2}\right)$. We see that the link configuration in Fig. 12 gives the intersection number 1 in Fig. 13.

\section{4D SO $(3)_{\theta=\pi}$ YANG-MILLS GAUGE THEORIES COUPLED TO THE BOUNDARY OF 5D SETS/ LONG-RANGE ENTANGLED TQFTS}

In Sec. II, we have shown that the SU(2) Yang-Mills theory with $\theta=\pi$, with the gauge bundle constraint $w_{2}\left(V_{\mathrm{PSU}(2)}\right)=$ $B+K_{1} w_{1}(T M)^{2}+K_{2} w_{2}(T M)$, has two distinct 't Hooft anomalies as shown in Eq. (2.38). In this section, we further comment on gauging the one-form $\mathbb{Z}_{2,[1]}^{e}$ center symmetry of the four sSiblings of $\mathrm{SU}(2)_{\theta=\pi} \mathrm{YM}$ to obtain $\mathrm{SO}(3)_{\theta=\pi} \mathrm{YM}$ theories. Since the 't Hooft anomalies involve the one-form center symmetry and the spacetime symmetries [whose background fields are the Stiefel-Whitney classes $\left.w_{i}(T M)\right]$, depending on which manifold we formulate the SU(2) YangMills, one obtains different theories.

\section{A. From $\mathbf{S U}(2)$ to $\mathbf{S O}(3)$ gauge theory}

To illustrate, we start by gauging the one-form symmetry $[6,78]$ of the $\mathrm{SU}(2)_{\theta=0}$ YM theories which is time-reversal symmetric and anomaly-free. There are still four choices of gauge bundle constraints labeled by $\left(K_{1}, K_{2}\right)$, i.e., Eq. (3.1). Let $\mathbf{Z}_{\mathrm{SU}(2) \mathrm{YM}}^{4 \mathrm{~d}}[B]$ be the path integral without specifying the gauge bundle constraint, the partition function with the gauge bundle constraint $w_{2}(E)=(B+$ $\left.K_{1} w_{1}(T M)^{2}+K_{2} w_{2}(T M)\right) \bmod 2$ is

$$
\mathbf{Z}_{\mathrm{SU}(2) \mathrm{YM}_{\left(K_{1}, K_{2}\right)}}^{4 \mathrm{~d}}[B] \equiv \int[D \Lambda] \mathbf{Z}_{\mathrm{SU}(2) \mathrm{YM}}^{4 \mathrm{~d}}[B] \exp \left(\mathrm{i} \pi \Lambda \cup\left(w_{2}(E)-\left(B+K_{1} w_{1}(T M)^{2}+K_{2} w_{2}(T M)\right)\right)\right) .
$$

More generally, we can add the counterterm $\frac{p \pi}{2} \mathcal{P}(B)$ labeled by an integer $p$, which modifies the partition function as

$$
\mathbf{Z}_{\mathrm{SU}(2) \mathrm{YM}_{\left(K_{1}, K_{2}\right)}}^{4 \mathrm{~d}}[B] \equiv \int[D \Lambda] \mathbf{Z}_{\mathrm{SU}(2) \mathrm{YM}}^{4 \mathrm{~d}}[B] \exp \left(\mathrm{i} \pi\left(\Lambda \cup\left(w_{2}(E)-\left(B+K_{1} w_{1}(T M)^{2}+K_{2} w_{2}(T M)\right)\right)+\frac{p}{2} \mathcal{P}(B)\right)\right) .
$$

Below we would like to obtain $\mathrm{SO}(3) \mathrm{YM}$ by gauging one-form $\mathbb{Z}_{2,[1]}^{e}$ center symmetry. The theta angle of the resulting theory is $2 \pi p$. If $w_{2}(T M)$ is nontrivial, the resulting $\mathrm{SO}(3)$ theory is time-reversal symmetric only when $p \in 2 \mathbb{Z}$ and $p \sim p+4$. When $w_{2}(T M)$ is trivial, the resulting $\mathrm{SO}(3)$ theory is time-reversal symmetric for $p \in \mathbb{Z}$ and $p \sim p+2$. In the following, we always restrict to the time-reversal symmetric case. Gauging one-form center symmetry amounts to summing over the background gauge field $B$ (promoting $B$ to a dynamical gauge field),

$$
\mathbf{Z}_{\mathrm{SO}(3) \mathrm{YM}_{\left(K_{1}, K_{2}\right)}^{4 \mathrm{~d}}}^{4}=\int[D \Lambda][D B] \mathbf{Z}_{\mathrm{SU}(2) \mathrm{YM}}^{4 \mathrm{~d}}[B] \exp \left(\mathrm{i} \pi\left(\Lambda \cup\left(w_{2}(E)-\left(B+K_{1} w_{1}(T M)^{2}+K_{2} w_{2}(T M)\right)\right)+\frac{p}{2} \mathcal{P}(B)\right)\right) .
$$

Integrating out $\Lambda$ enforces the relation between $\mathrm{SO}(3)$-gauge bundles and two-form dynamical gauge field $B$. This outputs the $\mathrm{SO}(3)$-gauge theory $\mathbf{Z}_{\mathrm{SO}(3)}^{4 \mathrm{~d}} \mathrm{YM}_{\left(K_{1}, K_{2}\right)}$ with $\theta=2 \pi p$.

\section{B. Gauging one-form $\mathbb{Z}_{2,[1]}^{e}$ symmetry of $\mathrm{SU}(2)$ gauge theory with $\theta=\pi$}

We proceed to discuss gauging the one-form symmetry of SU(2) Yang-Mills with $\theta=\pi$.
If one formulates the $\mathrm{SU}(2)_{\theta=\pi}$ Yang-Mills on an orientable and spin manifold, i.e., $w_{1}=w_{2}=0$ (hence $w_{3}=0$ as well), for spacetime tangent bundle $T M$, there is the freedom to ignore the time reversal as a symmetry of the theory. The only symmetry of interest is the one-form symmetry, which does not have an anomaly with itself. Hence one can gauge the one-form symmetry, and the resulting theory is $\mathrm{PSU}(2)=\mathrm{SO}(3)$ Yang-Mills with $\theta=\pi$. Indeed, $\mathrm{SO}(3)$ Yang-Mills with $\theta=\pi$ does not 
TABLE X. Possibilities of gauging the $\mathrm{SU}(2)_{\theta=\pi}$ Yang-Mills theory with gauge bundle constraint $\left(K_{1}, K_{2}\right)$ on a manifold with Stiefel-Whitney $(\mathrm{SW})$ class $\left(w_{1}, w_{2}\right)=\left(w_{1}(T M), w_{2}(T M)\right)$, where 0 and 1 mean trivial or nontrivial SW classes, respectively. The $\checkmark$ means that there is a way to make sense of the resulting gauged theory as a purely $4 \mathrm{~d}$ theory. The theories labeled by $\boldsymbol{X}$ mean that it only makes sense to discuss the combined $4 \mathrm{~d}-5 \mathrm{~d}$ systems. The WZW means the theory is intrinsically $4 \mathrm{~d}$; however, there is a WZW-like term of background fields, which involves a $5 \mathrm{~d}$ integral (but does not depend on the choice of $5 \mathrm{~d}$ manifold $M^{5}$ ).

\begin{tabular}{lcccc}
\hline \hline$\left(w_{1}, w_{2}\right) \backslash\left(K_{1}, K_{2}\right)$ & $(0,0)$ & $(1,0)$ & $(0,1)$ & $(1,1)$ \\
\hline$(0,0)$ & $\checkmark$ & $\checkmark$ & $\checkmark$ & $\checkmark$ \\
$(1,0)$ & $x$ & $x$ & $x$ & $x$ \\
$(0,1)$ & $\checkmark$ & $\checkmark$ & WZW & WZW \\
$(1,1)$ & $x$ & $x$ & $x$ & $x$ \\
\hline \hline
\end{tabular}

respect time reversal, which maps $\theta=\pi$ to $\theta=3 \pi$ due to the identification $\theta \sim \theta+4 \pi$ on a spin manifold.

If one formulates the $\mathrm{SU}(2)_{\theta=\pi}$ Yang-Mills on an orientable and nonspin manifold, one still has the freedom to ignore the time reversal as a symmetry of the theory. However, in this case, there is a counterterm

$$
\int_{M^{5}} K_{2} \pi \mathrm{Sq}^{1}\left(w_{2}(T M) \cup B\right),
$$

which is a WZW-like term of background fields (i.e., probe fields in condensed matter language). Denoting the partition function of the $\mathrm{SU}(2)_{\theta=\pi}$ Yang-Mills coupled to $B$ as $\mathbf{Z}_{\mathrm{SU}(2) \mathrm{YM}_{\left(0, K_{2}\right)}}\left[M^{4}, B\right]$, after promoting $B$ to a dynamical field, the partition function of the entire $4 d-5 d$ system is

$$
\begin{aligned}
& \int[D B] \mathbf{Z}_{\mathrm{SU}(2) \operatorname{YM}_{\left(0, K_{2}\right)}}\left[M^{4}, B\right] \\
& \quad \times \exp \left(\mathrm{i} \pi \int_{M^{5}} K_{2} \pi \operatorname{Sq}^{1}\left(w_{2}(T M) \cup B\right)\right) .
\end{aligned}
$$

If $K_{2}=0$, the $4 \mathrm{~d}-5 \mathrm{~d}$ system reduces to an intrinsic $4 \mathrm{~d}$ system. Physically, this corresponds to the case where the gauge charge is a boson. It makes sense to gauge the oneform symmetry which again gives rise to the time-reversal broken $\mathrm{SO}(3)$ Yang-Mills theory. If $K_{2}=1$, the theory is still an intrinsic 4d system. Physically, this corresponds to the case where the gauge charge is a fermion.

If one formulates the SU(2) Yang-Mills on an unorientable manifold, the time-reversal symmetry is built in, so time-reversal symmetry is too late to be abandoned. Promoting $B$ to a dynamical gauge field, the partition function for the entire $4 d-5 d$ system is

$$
\begin{aligned}
& \int[D B] \mathbf{Z}_{\mathrm{SU}(2) \mathrm{YM}_{\left(K_{1}, K_{2}\right)}}\left[M^{4}, B\right] \exp \left[\mathrm { i } \pi \int _ { M ^ { 5 } } \left(B \mathrm{Sq}^{1} B+\mathrm{Sq}^{2} \mathrm{Sq}^{1} B\right.\right. \\
& \left.\left.+K_{1} w_{1}(T M)^{2} \mathrm{Sq}^{1} B+K_{2} \mathrm{Sq}^{1}\left(w_{2}(T M) \cup B\right)\right)\right]
\end{aligned}
$$

Since $M^{5}$ is unorientable, for all four choices of $\left(K_{1}, K_{2}\right)$, the $5 \mathrm{~d}$ terms do not vanish (because $B \mathrm{Sq}^{1} B+\mathrm{Sq}^{2} \mathrm{Sq}^{1} B$ is always nonvanishing on an unorientable manifold). Hence one can only discuss the $4 \mathrm{~d}-5 \mathrm{~d}$ system rather than discuss the $4 \mathrm{~d}$ system alone. We summarize all the above cases in Table X.

\section{LATTICE REGULARIZATION, UV COMPLETION, AND SYMMETRIC ANOMALOUS TQFT}

In this section, we formulate the partition function of the $5 \mathrm{~d}$ higher-SPT $\mathbf{Z}_{\mathrm{SPT}_{\left(K_{1}=0, K_{2}=0\right)}^{5 \mathrm{~d}}}^{5}\left[M^{5} ; B\right]$ on a simplicial complex spacetime. This provides a lattice regularization of the $5 \mathrm{~d}$ SPT. We also provide lattice realization of (i) $4 \mathrm{~d}$ higher-symmetry-extended boundary theory or (ii) 4d higher-symmetry-enriched anomalous topologically ordered boundary theory. We will generalize the approach in [54] and follow Sec. IX of [55]. In condensed matter physics, this (ii) phenomenon is known as the anomalous surface topological order (first noticed in [79]) typically for the $2+1 \mathrm{D}$ boundary of $3+1 \mathrm{D}$ SPTs; see a review [30].

\section{A. Lattice realization of $4 d$ higher SPTs and higher-gauge TQFT: 4d simplicial complex and $3+1 D$ condensed matter realization}

We warm up by considering a lattice realization of $4 d$ higher SPTs given by a probe-field partition function

$$
\begin{aligned}
\mathbf{Z}_{\mathrm{SPT}}^{4 \mathrm{~d}}\left[M^{4} ; B\right] & =\exp \left(\mathrm{i} \frac{\pi}{2} \int_{M^{4}} \mathcal{P}(B)\right) \\
& =\exp \left(\mathrm{i} \frac{\pi}{2} \int_{M^{4}} B \cup B+\underset{1}{\cup} \delta B\right) .
\end{aligned}
$$

The path integral can be regularized on a triangulated four manifold $M^{4}$. The building blocks of $M^{4}$ are foursimplices. Without loss of generality, we consider an arbitrary four-simplex which we denote as (01234) where each number labels one vertex. See Fig. 14 for a graphical representation of a four-simplex. We denote $B_{i j k}$ as restricting the two-cochain $B$ on the two-simplex $(i j k)$. We label the path integral amplitude on (01234) as $\omega_{4}(01234)$, i.e.,

$$
\begin{aligned}
\omega_{4}(01234)= & \exp \left[\mathrm{i} \frac{\pi}{2}(B \cup B+B \cup \delta B)_{01234}\right] \\
= & \exp \left[\mathrm { i } \frac { \pi } { 2 } \left(B_{012} B_{234}\right.\right. \\
& +B_{034}\left(B_{123}-B_{023}+B_{013}-B_{012}\right) \\
& \left.\left.+B_{014}\left(B_{234}-B_{134}+B_{124}-B_{123}\right)\right)\right] .
\end{aligned}
$$




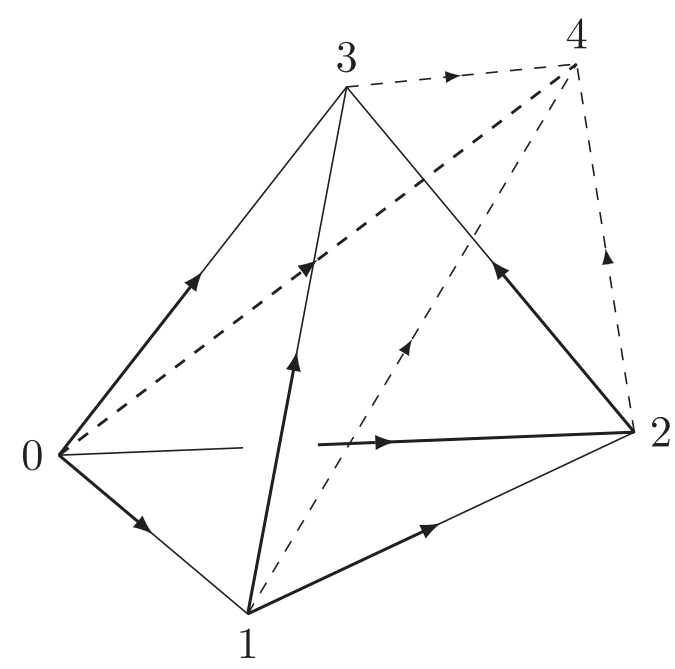

FIG. 14. Graphical representation of a four-simplex (01234).

It is straightforward to verify that $\omega_{4}(01234)$ satisfies the cocycle condition:

$\left(\delta \omega_{4}\right)(012345)=\frac{\omega_{4}(12345) \cdot \omega_{4}(01345) \cdot \omega_{4}(01235)}{\omega_{4}(02345) \cdot \omega_{4}(01245) \cdot \omega_{4}(01234)}=1$.

\section{B. Lattice realization of $5 d$ higher SPTs and higher-gauge SETs: 5d simplicial complex and $4+10$ condensed matter realization}

The $5 \mathrm{~d}$ partition function with $\left(K_{1}=0, K_{2}=0\right)$ is

$\mathbf{Z}_{\mathrm{SPT}_{\left(K_{1}=0, K_{2}=0\right)}}^{5 \mathrm{~d}}\left[M^{5}\right]=\exp \left(\mathrm{i} \pi \int_{M^{5}} B \mathrm{Sq}^{1} B+\mathrm{Sq}^{2} \operatorname{Sq}^{1} B\right)$.

We start by triangulating the $5 \mathrm{~d}$ closed spacetime manifold (without boundary) into a five-simplicial complex. There are some useful identities:

$$
\begin{aligned}
\mathrm{Sq}^{1} B & =\underset{1}{B \cup B}=\frac{1}{2} \delta B, \\
\mathrm{Sq}^{2} \mathrm{Sq}^{1} B & =\left(\mathrm{Sq}^{1} B\right) \underset{1}{\cup}\left(\mathrm{Sq}^{1} B\right)=\frac{1}{4}(\delta B) \underset{1}{\cup}(\delta B) .
\end{aligned}
$$

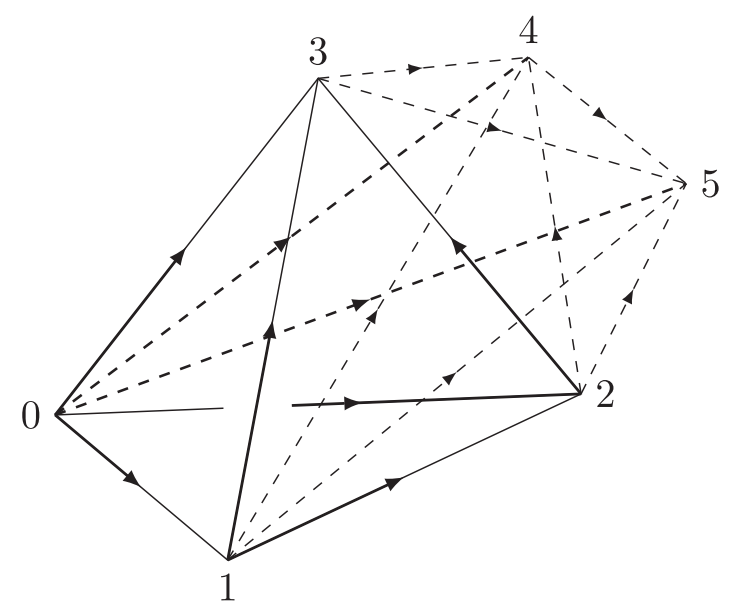

FIG. 15. Graphical representation of a five-simplex (012345).

Note that in the second equality of the first line, we have used the cocycle condition that $\delta B=0 \bmod 2$. One can express the SPT action Eq. (8.4) in terms of the sum of cup products of $B$ cochains over five-simplices

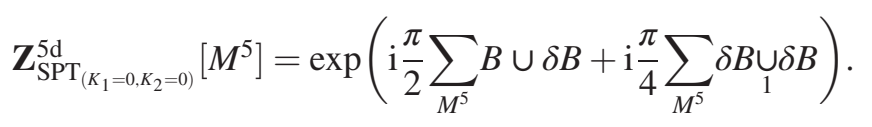

Without loss of generality, we consider an arbitrary fivesimplex which we denote as $(012345)$ where each number labels one vertex. See Fig. 15 for a graphical representation of a five-simplex. We will label the path integral amplitude on the simplex $(012345)$ as $\omega_{5}(012345)$, i.e.,

$$
\omega_{5}(012345)=\exp \left[\mathrm{i} \pi\left(\frac{1}{2} B \cup \delta B+\frac{1}{4} \delta B \cup \delta B\right)_{012345}\right],
$$

so that the partition function can be simplified as $\mathbf{Z}_{\mathrm{SPT}_{\left(K_{1}=0, K_{2}=0\right)}^{5 \mathrm{~d}}}\left[M^{5}\right]=\prod_{(i j k l m n) \in M^{5}} \omega(i j k l m n)$. Using the definition of the cup products on simplices and the identities Eq. (8.5), we have

$$
\begin{aligned}
\left(\mathrm{Sq}^{1} B\right)_{0123}= & \frac{1}{2}\left(B_{123}-B_{023}+B_{013}-B_{012}\right) \\
\left(\mathrm{Sq}^{2} \mathrm{Sq}^{1} B\right)_{012345}= & \frac{1}{4}\left((\delta B)_{0345}(\delta B)_{0123}+(\delta B)_{0145}(\delta B)_{1234}+(\delta B)_{0125}(\delta B)_{2345}\right) \\
= & \frac{1}{4}\left(\left(-B_{045}-B_{034}+B_{035}+B_{345}\right)\left(-B_{023}-B_{012}+B_{013}+B_{123}\right)\right. \\
& +\left(-B_{045}-B_{014}+B_{015}+B_{145}\right)\left(-B_{134}-B_{123}+B_{124}+B_{234}\right) \\
& \left.+\left(-B_{025}-B_{012}+B_{015}+B_{125}\right)\left(-B_{245}-B_{234}+B_{235}+B_{345}\right)\right)
\end{aligned}
$$


Hence the path integral amplitude on the simplex (012345) is

$$
\begin{aligned}
\omega_{5}(012345)= & \exp \left[\frac{\mathrm{i} \pi}{2} B_{012}\left(-B_{245}-B_{234}+B_{235}+B_{345}\right)\right. \\
& +\frac{\mathrm{i} \pi}{4}\left(-B_{045}-B_{034}+B_{035}+B_{345}\right)\left(-B_{023}-B_{012}+B_{013}+B_{123}\right) \\
& +\frac{\mathrm{i} \pi}{4}\left(-B_{045}-B_{014}+B_{015}+B_{145}\right)\left(-B_{134}-B_{123}+B_{124}+B_{234}\right) \\
& \left.+\frac{\mathrm{i} \pi}{4}\left(-B_{025}-B_{012}+B_{015}+B_{125}\right)\left(-B_{245}-B_{234}+B_{235}+B_{345}\right)\right] .
\end{aligned}
$$

It is straightforward to verify that $\omega_{5}(012345)$ satisfies the cocycle condition:

$$
\left(\delta \omega_{5}\right)(0123456)=\frac{\omega_{5}(123456) \cdot \omega_{5}(013456) \cdot \omega_{5}(012356) \cdot \omega_{5}(012345)}{\omega_{5}(023456) \cdot \omega_{5}(012456) \cdot \omega_{5}(012346)}=1
$$

We emphasize that $\omega(012345)$ is a cocycle only when $B$ is a cocycle, i.e., $\delta B=0$. If $B$ is a cochain rather than a cocycle, Eq. (8.4) is not a cocycle, and hence it cannot be a partition function of a topological field theory. ${ }^{44}$

We further comment on the lattice regularization of theory with various choices of $\left(K_{1}, K_{2}\right)$ :

(1) When $\left(K_{1}, K_{2}\right)=(0,0)$, as we derived above, there is a lattice regularization of the $5 \mathrm{~d}$ SPT partition function.

(2) When $\left(K_{1}, K_{2}\right)=(1,0)$, the path integral amplitude depends on the first Stiefel-Whitney class $w_{1}(T M)$. Using the method of [29], one can write down the simplicial form of $w_{1}(T M)^{2}$ using the twisted cocycle, with the coefficient in $U(1)_{T}$ due to the antiunitary nature of time-reversal symmetry (in the Hamiltonian formalism of [29]). We will not write down the explicit expression for the cocycle.

(3) $\left(K_{1}, K_{2}\right)=(0,1),(1,1)$ has the same anomaly polynomial as $\left(K_{1}, K_{2}\right)=(0,0),(1,0)$, respectively.

Other than treating $B$ as the background gauge field, we can also sum over $B$ to get the topologically ordered $5 \mathrm{~d}$ SET $\mathbf{Z}_{\mathrm{SET}_{\left(K_{1}, K_{2}\right)}^{5 \mathrm{~d}}}^{\mathrm{an}}\left[M^{5}\right]$.

Given that the 5d SPT and 5d SET path integral can be regularized on a lattice, following [29], one can write down the quantum wave function via the spacetime path integral. It is also possible to construct a lattice quantum Hamiltonian on the 4D space (on a constant time slice), for both SPTs and SETs, similar to the formulations of [29,80-82]. For the topologically ordered 5d SET, we implement the method of $[81,82]$

\footnotetext{
${ }^{44}$ The cocycle condition is crucial in proving the partition function to be invariant under retriangulating the spacetime manifold $M^{5}$.
}

$$
\hat{\mathbf{H}}=-\sum_{1 \text {-link } \ell} \hat{\mathbf{A}}_{\ell}-\sum_{\text {3-simplex }} \hat{\mathbf{B}}_{3 \text {-simplex }}
$$

where $\hat{\mathbf{A}}_{\ell}$ is an operator acting on the plaquettes (twosimplex) adjacent to the one-link $\ell$, and $\hat{\mathbf{B}}_{3 \text {-simplex }}$ is an operator acting on the boundary of a given three-simplex which again are plaquettes (two-simplex). The $\hat{\mathbf{A}}_{\ell}$ has its effect on imposing the time evolution constraint as the same as the path integral formulation: $\hat{\mathbf{A}}_{\ell}$ lifting the state vector to a next time slice locally around the one-link $\ell$. The $\hat{\mathbf{B}}_{3 \text {-simplex }}$ imposes the zero flux condition enclosed by the three-simplex (which is a two-sphere $S^{2}$ in topology). We will not give the explicit expression of the quantum Hamiltonian $\hat{\mathbf{H}}$ in this work.

\section{Higher-symmetry-extended 3 + 1D gapped boundaries}

One option to saturate the anomaly inflow from the bulk $5 \mathrm{~d}(4+1 \mathrm{D})$ SPT is to extend the global symmetry on the $4 \mathrm{~d}$ $(3+1 D)$ boundary, based on the symmetry-extension method of [54]. Note that $\left(K_{1}, K_{2}=0\right)$ and $\left(K_{1}, K_{2}=\right.$ 1) theories have the same $4 d$ anomaly, but they differ by a $4 \mathrm{~d}$ WZW-like counterterm written on a $5 \mathrm{~d} M^{5}$,

$$
\begin{aligned}
\mathbf{Z}_{\mathrm{SPT}_{\left(K_{1}, K_{2}\right)}}^{5 \mathrm{~d}}\left[M^{5}\right]= & \mathbf{Z}_{\mathrm{SPT}_{\left(K_{1}, 0\right)}}^{\mathrm{sd}}\left[M^{5}\right] \\
& \times \exp \left(\mathrm{i} \pi \int_{M^{5}} K_{2} \operatorname{Sq}^{1}\left(w_{1}(T M)^{2} B\right)\right) .
\end{aligned}
$$

The $4 \mathrm{~d}$ counterterm is shown to be vanished on a closed $M^{5}$ in $5 \mathrm{~d}$ in Eq. (2.33). Due to Eq. (2.33), this $K_{2}$-dependent term has no consequences via any anomaly consideration on $4 \mathrm{~d}$ dynamics. Thus we only discuss the $3+1 \mathrm{D}$ gapped boundary for the two siblings $\left(K_{1}, K_{2}=0\right)$. The $5 \mathrm{~d}$ partition function is 


$$
\begin{aligned}
\mathbf{Z}_{\mathrm{SPT}_{\left(K_{1}, 0\right)}}^{5 \mathrm{~d}}\left[M^{5}\right] & =\exp \left(\mathrm{i} \pi \int_{M^{5}} B \mathrm{Sq}^{1} B+\mathrm{Sq}^{2} \mathrm{Sq}^{1} B+K_{1} w_{1}(T M)^{2} \mathrm{Sq}^{1} B\right) \\
& =\exp \left(\mathrm{i} \pi \int_{M^{5}}\left(B+\left(1+K_{1}\right) w_{1}(T M)^{2}+w_{2}(T M)\right) \cup \mathrm{Sq}^{1} B\right) \cdot \exp \left(\mathrm{i} \pi \int_{M^{5}} \delta(\cdots)\right) .
\end{aligned}
$$

In the second line, we have used $\int_{M^{5}} \mathrm{Sq}^{2} \mathrm{Sq}^{1} B=$ $\int_{M^{5}}\left(w_{1}(T M)^{2}+w_{2}(T M)\right) \mathrm{Sq}^{1} B+\int_{M^{5}} \delta(\cdots)$. Note that when $M^{5}$ is closed, the total derivative vanishes and we have the standard $\mathrm{Wu}$ formula $\int_{M^{5}} \mathrm{Sq}^{2} \mathrm{Sq}^{1} B=$ $\int_{M^{5}}\left(w_{1}(T M)^{2}+w_{2}(T M)\right) \mathrm{Sq}^{1} B$. When $M^{5}$ has a boundary, the standard $\mathrm{Wu}$ formula may no longer hold, and they differ at most by a coboundary term. ${ }^{45}$ We denote the coboundary term as $\delta(\cdots)$ in the second line. Since $(\cdots)$ is a well-defined term of background gauge fields, it is a $4 d$ invertible TQFT, which does not contribute to the $4 \mathrm{~d}$ dynamics. Hence in the construction of $4 \mathrm{~d}$ symmetric boundary TQFT below, we only focus on the first part in the second line of Eq. (8.12). Using the systematic way in [54] and its generalized higher-symmetry extension [55], we find that the boundary of 5d SPT can support a $4 \mathrm{~d}$ TQFT via the higher-symmetry extension from a oneform $\mathbb{Z}_{2}$ to a one-form $\mathbb{Z}_{4}$ symmetry. Schematically, let $\omega_{5}^{\left(K_{1}, 0\right)}$ be the five-cocycle whose product over the $5 \mathrm{~d}$ manifold $M^{5}$ gives the 5d SPT partition function Eq. (8.12). Let $\beta_{4}^{\left(K_{1}, 0\right)}$ be a four-cochain which trivializes the $5 \mathrm{~d}$ cocycle, i.e.,

$$
\omega_{5}^{\left(K_{1}, 0\right)}=\delta \beta_{4}^{\left(K_{1}, 0\right)}
$$

$$
\begin{array}{cc}
\mathrm{B}^{2} \mathbb{Z}_{2} \longrightarrow \quad & \mathrm{B}^{2} \mathbb{Z}_{4} \\
& (\text { Extended global symmetry } \\
& \mathbb{Z}_{4} \text { one-form symmetry probe } \\
& \text { by } \mathbb{Z}_{4} \text { two-cochain } C \text { background field) }
\end{array}
$$

This higher-symmetry-extended 3 + 1D gapped boundary, described by (8.14), has no long-range entanglement and no intrinsic topological order. This higher-symmetryextended 3+1D gapped boundary is known as system (i) in Sec. 7 of Ref. [13] as a short-range entangled state,

\footnotetext{
${ }^{45} \mathrm{By}$ the Wu formula for $n$ manifolds with boundary, $\mathrm{Sq}^{j} x=$ $u_{j} x$ where $u_{j}$ is the relative Wu class, and $x \in H^{n-j}\left(M, \partial M ; \mathbb{Z}_{2}\right)$. The total relative Wu class $u$ is related to the total Stiefel-Whitney class $w$ of $M$ as $\operatorname{Sq}(u)=w$. So $u_{1}=w_{1}(T M), u_{2}=w_{1}(T M)^{2}+$ $w_{2}(T M)$, etc., still hold but $\mathrm{Sq}^{1} B$ may not be in $H^{3}\left(M, \partial M ; \mathbb{Z}_{2}\right)$ for a five manifold $M$ with boundary. Therefore, $\mathrm{Sq}^{2} \mathrm{Sq}^{1} B=$ $\left(w_{1}(T M)^{2}+w_{2}(T M)\right) \mathrm{Sq}^{1} B$ may not hold for a five manifold $M$ with boundary. See for instance Ref. [83].
}

We find that the following $\beta_{4}^{\left(K_{1}, 0\right)}$ satisfies Eq. (8.13):

$$
\begin{aligned}
\beta_{4}^{\left(K_{1}, 0\right)}= & \exp \left[\mathrm { i } \pi \int _ { M ^ { 4 } } \left(B+\left(1+K_{1}\right) w_{1}(T M)^{2}\right.\right. \\
& \left.\left.+w_{2}(T M)\right) \cup \gamma(C)\right]
\end{aligned}
$$

where $C$ is a $\mathbb{Z}_{4}$ valued two-cochain satisfying $B=$ $C \bmod 2$ and $\gamma: \mathbb{Z}_{4} \rightarrow \mathbb{Z}_{2}$ is a function which maps the $\mathbb{Z}_{4}$ two-cochain to a $\mathbb{Z}_{2}$ two-cochain:

$$
(\gamma(C))_{i j k}=\frac{\left(C_{i j k}\right)^{2}-C_{i j k}}{2} .
$$

In summary, the $\beta_{4}^{\left(K_{1}, 0\right)}$ in (8.14) is the partition function of the higher-symmetry-extended 3+1D gapped boundary, while the original one-form anomalous global symmetry $\mathbb{Z}_{2,[1]}$ is extended to the anomaly-free one-form global symmetry $\mathbb{Z}_{4,[1]}$. We can also rephrase that the higher anomaly associated with the bordism group $\Omega_{5}^{\mathrm{O}}\left(\mathrm{B}^{2} \mathbb{Z}_{2}\right)$ with a higher classifying space $\mathrm{B}^{2} \mathbb{Z}_{2}$ can be pulled back and trivialized as fully anomaly-free in the bordism group $\Omega_{5}^{\mathrm{O}}\left(\mathrm{B}^{2} \mathbb{Z}_{4}\right)$ with an extended higher classifying space $B^{2} \mathbb{Z}_{4}$ :

$$
\begin{gathered}
\mathrm{B}^{2} \mathbb{Z}_{2} \\
(\text { Global Symmetry } \\
\mathbb{Z}_{2} \text { one-form symmetry probe } \\
\text { by } \mathbb{Z}_{2} \text { two-cochain } B \text { background field) }
\end{gathered}
$$

both in the bulk and on the boundary (denoted as SRE/SRE in Sec. 7 of Ref. [13]). In fact, this whole SRE/SRE bulk-boundary theory is still an invertible TQFT with a partition function $|\mathbf{Z}|=1$ on an $M^{5}$ with a $4 \mathrm{~d}$ gapped boundary $M^{4}$.

\section{Higher-symmetry anomalous 3+1D topologically ordered gapped boundaries: Spontaneous higher-symmetry breaking}

There also exists another boundary theory of the $5 \mathrm{~d}$ SPTs in Eq. (8.12), with $\mathbb{Z}_{2}$ two-cochain $b$ summed over as dynamical fields on the boundary. The boundary theory is a dynamical $\mathbb{Z}_{2}$ gauge theory, which can be obtained from gauging the normal $\mathbb{Z}_{2,[1]}$ subgroup of $\mathbb{Z}_{4,[1]}$ in the 
symmetry extended gapped boundary from the previous section. Schematically, we promote the two-form (or twocochain) gauge field $b$ coupling to one-form $\mathbb{Z}_{2,[1]}$ symmetry to dynamical, in a normal subgroup of $\mathbb{Z}_{4,[1]}$. The resulting boundary theory has long-range entanglement in contrast to the short-range entanglement of the symmetric extended boundary theory in the previous section, Sec. VIII C. This can be summarized as an induced fiber sequence of their higher classifying space $\mathrm{B}^{2} \mathbb{Z}_{2} \rightarrow$ $\mathrm{B}^{2} \mathbb{Z}_{4} \rightarrow \mathrm{B}^{2} \mathbb{Z}_{2}$ :

\author{
$\mathrm{B}^{2} \mathbb{Z}_{2} \quad \rightarrow \quad \mathrm{B}^{2} \mathbb{Z}_{4} \rightarrow$ \\ (Dynamical/emergent \\ gaugeable \\ $\mathbb{Z}_{2}$ two-cochain $b$ field)
}

$$
\begin{gathered}
\mathrm{B}^{2} \mathbb{Z}_{2} \\
(\text { Global Symmetry } \\
\mathbb{Z}_{2,[1]} \text { symmetry probed by } \\
\left.\mathbb{Z}_{2} \text { two-cochain background } B \text { field }\right)
\end{gathered}
$$

Or we may denote the above as $\mathrm{B}^{2} \mathbb{Z}_{2}^{\text {gauged }} \rightarrow \mathrm{B}^{2} \mathbb{Z}_{4}^{\text {total }} \rightarrow$ $\mathrm{B}^{2} \mathbb{Z}_{2}^{G}$, implementing the notations of $[13,54]$.

However, as noticed in Ref. [54] and in Sec. 7 of Ref. [13], when the boundary theory is long-range entangled (after gauging a normal subgroup), it is possible that the new fate of low energy dynamics may not preserve the global symmetry. Specifically, Ref. [54] finds that, under the exact sequence $K \rightarrow H \rightarrow G$, even for a successful $H$-symmetry extended construction of gapped boundary, in certain cases, dynamically gauging the normal subgroup $K$ may still result in $G$ spontaneously broken. In short, we should question:

Is it possible that $\mathbb{Z}_{2,[1]}$ global symmetry in the longrange entangled boundary theory happens to be spontaneously broken?

Namely, following the notations in Sec. 7 of Ref. [13] on the SRE/SRE and SRE/LRE of the bulk/boundary (denoted bulk/bdry) systems, the symmetry-extension construction $[13,54]$ under the exact sequence $K \rightarrow H \rightarrow G$ may result in different dynamical fates:

$\left\{\begin{array}{l}\text { Case } 1 . H \text {-symmetry-extended gapped boundary: SRE/SRE bulk/bdry. } \\ \text { e.g., The one-form } \mathbb{Z}_{4,[1]} \text { symmetry extended in Sec. } 8.3 \text {. Many zero-form } G \text {-symmetry examples given in Ref. [53]. } \\ \text { Case 2. } G \text {-symmetry-preserving anomalous } K \text {-gauge gapped boundary: SRE/LRE bulk/bdry. } \\ \text { e.g., Many zero-form } G \text {-symmetry examples given in Ref. [53]. } \\ \text { Case 3. } G \text {-symmetry-breaking } K \text {-gauge gapped boundary: SRE/LRE bulk/bdry. } \\ \text { e.g., Sec. } 3.4 \text { and Appendix A.2.4 of Ref. [53], and Sec. } 7.1 \text { of Ref. [12]. }\end{array}\right.$

Thus below what we aim to examine is whether a proposal of Case 2 associated with Eq. (8.17) is in fact the Case 3 in disguise, when $K$ is dynamically gauged, as

$$
\begin{array}{ccc}
\mathrm{B}^{2} \mathbb{Z}_{2} & \rightarrow \mathrm{B}^{2} \mathbb{Z}_{4} \rightarrow & \mathrm{B}^{2} \mathbb{Z}_{2} \\
\text { (Dynamical/emergent } & & \text { (Global Symmetry } \\
\text { gaugeable } & \mathbb{Z}_{2,[1]} \text { symmetry } \\
\mathbb{Z}_{2} \text { two-cochain } b \text { field) } & \text { spontaneously broken?) }
\end{array}
$$

Concretely, we propose a $\mathbb{Z}_{2}$ gauge theory [as a candidate IR theory of the UV SU(2) YM] which has $\mathbb{Z}_{2,[1]}$ global symmetry and saturates the 't Hooft anomaly Eq. (8.12). We will find that the $\mathbb{Z}_{2,[1]}$ global symmetry is spontaneously broken.

We consider the $4 \mathrm{~d} \mathbb{Z}_{2}$-gauge TQFT

$$
\begin{aligned}
& \int {[D b][D \tilde{a}] \exp \left(\mathrm{i} \pi \int_{M^{4}} b \delta \tilde{a}+\frac{1}{4} \mathcal{P}(\delta \tilde{a})\right) } \\
&=\int[D b][D \tilde{a}] \exp \left(\mathrm{i} \pi \int_{M^{4}} b \delta \tilde{a}+\tilde{a} \tilde{a} \tilde{a} \tilde{a}\right) .
\end{aligned}
$$

Here $\int[D b][D \tilde{a}]$ means we sum over the $\mathbb{Z}_{2}$ valued twocochain $b \in C^{2}\left(M, \mathbb{Z}_{2}\right)$ and $\mathbb{Z}_{2}$ valued one-cochain $\tilde{a} \in C^{1}\left(M, \mathbb{Z}_{2}\right)$. The first term is the standard BF term of discrete gauge theory, while the second term is the $4 \mathrm{~d}$ analogue of the Dijkgraaf-Witten-type action [12]. We also refer to it as the twisting term. Integrating out $b$ enforces $\tilde{a}$ to be a $\mathbb{Z}_{2}$ valued cocycle, and hence the twisting term 
$\frac{1}{4} \mathcal{P}(\delta \tilde{a})=\frac{1}{4} \delta \tilde{a} \delta \tilde{a}=\tilde{a} \tilde{a} \tilde{a} \tilde{a}$ mod 2 is time-reversal symmetric. To match the anomaly from 5d SPTs in Eq. (8.12), we couple to background fields $B$ and $w_{1}(T M)$, as follows:

$$
\int_{M^{4}} \pi b\left(\delta \tilde{a}-B-K_{1} w_{1}(T M)^{2}\right)+\frac{\pi}{4} \mathcal{P}(\delta \tilde{a}-B) .
$$

Summing over the $\mathbb{Z}_{2}$ valued two-cochain $b$ enforces the gauge bundle constraint:

$$
\delta \tilde{a}=B+K_{1} w_{1}(T M)^{2} \bmod 2 .
$$

Notice that under the gauge transformation of the background field $B \rightarrow B+\delta \lambda$, we demand $\tilde{a} \rightarrow \tilde{a}+\lambda$. It is obvious that the action Eq. (8.20) is gauge invariant. However, due to the gauge bundle constraint Eq. (8.21), the twisting term $\frac{\pi}{4} \mathcal{P}(\delta \tilde{a}-B)$ is no longer 0 or $\pi \bmod 2 \pi$; hence it is not time-reversal invariant, and Eq. (8.20) is not well defined on an unorientable manifold. To make sense of the theory on an unorientable manifold, we use the same idea in Sec. II where we promote the twisting term to a $5 \mathrm{~d}$ integral,

$$
\begin{aligned}
\int_{M^{5}} \frac{\pi}{4} \delta \mathcal{P}(\delta \tilde{a}-B)= & \int_{M^{5}} \frac{\pi}{2} \delta \tilde{a} \delta(\delta \tilde{a})+\frac{\pi}{2} \delta B \delta \tilde{a}+\frac{\pi}{4} \delta \mathcal{P}(B) \\
= & \pi \int_{M^{5}} B \mathrm{Sq}^{1} B+\mathrm{Sq}^{2} \mathrm{Sq}^{1} B \\
& +K_{1} w_{1}(T M)^{2} \mathrm{Sq}^{1} B
\end{aligned}
$$

where we have used $\frac{\pi}{4} \delta \mathcal{P}(B)=\pi\left(B \mathrm{Sq}^{1} B+\mathrm{Sq}^{2} \mathrm{Sq}^{1} B\right)$ and the gauge bundle constraint Eq. (8.21). Thus when we couple theory to the background field $B$ and formulate it on an unorientable manifold, only the $4 \mathrm{~d}-5 \mathrm{~d}$ coupled system is well defined,

$$
\begin{aligned}
& \int[D b][D \tilde{a}] \exp \left(\mathrm{i} \pi \int_{M^{4}} \pi b\left(\delta \tilde{a}-B-K_{1} w_{1}(T M)^{2}\right)\right. \\
& \left.+\mathrm{i} \pi \int_{M^{5}} B \operatorname{Sq}^{1} B+\operatorname{Sq}^{2} \operatorname{Sq}^{1} B+K_{1} w_{1}(T M)^{2} \mathrm{Sq}^{1} B\right) .
\end{aligned}
$$

Indeed, the $5 \mathrm{~d}$ integral implies that the $\mathbb{Z}_{2}$ gauge theory saturates the anomaly Eq. (8.12). Let us comment on the dynamics of the above $4 \mathrm{~d} \mathbb{Z}_{2}$ gauge theory. The fact that $\tilde{a}$ transforms as $\tilde{a} \rightarrow \tilde{a}+\lambda$ under one-form background gauge transformation $B \rightarrow B+\delta \lambda$ suggests that the Wilson line

$$
\exp (\mathrm{i} \pi \oint \tilde{a})
$$

has charge 1 under $\mathbb{Z}_{2,[1]}$. In a TQFT, any genuine line operators are tensionless, and thus the expectation does not satisfy area law. Since $\exp (\mathrm{i} \pi \oint \tilde{a})$ is a genuine line operator, it obeys a perimeter law, and it spontaneously breaks the $\mathbb{Z}_{2,[1]}$ one-form symmetry.

We comment that in Sec. 7 of Ref. [13], similar arguments have been used to show that the zero-form $\mathbb{Z}_{2}$ global symmetry of a $2 \mathrm{~d}$ TQFT is spontaneously broken. In the $2 \mathrm{~d}$ theory, there is a scalar $\phi$ with charge 1 under $\mathbb{Z}_{2}$ zero-form global symmetry. In the $2 \mathrm{~d}$ TQFT, $\phi$ has a nontrivial expectational value. Thus the $\mathbb{Z}_{2}$ zero-form symmetry is spontaneously broken. See Ref. [13] for more details.

The fact that the dynamical $\mathbb{Z}_{2}$ gauge theory we constructed spontaneously breaks the one-form $\mathbb{Z}_{2,[1]}$ symmetry is consistent with the conclusion pointed out by Cordova and Ohmori (see $[84,85]$ ) where the authors showed the impossibility of having any $\mathbb{Z}_{2}^{T} \times \mathbb{Z}_{2,[1]}$ symmetric anomalous gapped 4d TQFT saturating the $4 d$ higher anomaly of 5d SPTs Eq. (8.12).

\section{CONCLUSIONS, DISCUSSIONS, AND DYNAMICS}

(1) Summary.-In this work, we proved (physically via the quantum field theory method) that $4 \mathrm{~d}$ timereversal symmetric pure YMs of an SU(2) gauge group with a second-Chern-class topological term at $\theta=\pi$ [i.e., $\mathrm{SU}(2)_{\theta=\pi} \mathrm{YM}$ ] have new higher 't Hooft anomalies in $4 \mathrm{~d}$, given by a $5 \mathrm{~d}$ topological term Eq. (2.38) and Eq. (2.61):

$$
\begin{aligned}
& \pi \int_{M^{5}}\left(B \cup \mathrm{Sq}^{1} B+\mathrm{Sq}^{2} \mathrm{Sq}^{1} B+K_{1} w_{1}(T M)^{2} \cup \mathrm{Sq}^{1} B\right. \\
& \left.\quad+K_{2} \mathrm{Sq}^{1}\left(w_{2}(T M) \cup B\right)\right) .
\end{aligned}
$$

The $5 \mathrm{~d}$ term $B \mathrm{Sq}^{1} B+\mathrm{Sq}^{2} \mathrm{Sq}^{1} B+K_{1} w_{1}(T M)^{2} \mathrm{Sq}^{1} B$ is a $5 \mathrm{~d}$ bordism invariant (or equivalently $5 \mathrm{~d}$ iTQFT/ SPTs/counterterm) that specifies the $4 \mathrm{~d}$ 't Hooft anomaly. However, the $5 \mathrm{~d}$ term $K_{2} \mathrm{Sq}^{1}\left(w_{2}(T M) B\right)$ is not a $5 \mathrm{~d}$ bordism invariant but only a $4 \mathrm{~d}$ WZW-like counterterm; thus strictly speaking it does not indicate any $4 \mathrm{~d}$ 't Hooft anomaly. We found that there are at least four siblings of $\mathrm{SU}(2)_{\theta=\pi}$ YM with bosonic UV completion, labeled by $\left(K_{1}, K_{2}\right) \in\left(\mathbb{Z}_{2}, \mathbb{Z}_{2}\right)$. Their higher 't Hooft anomalies of generalized global symmetries indicate that $4 \mathrm{~d} \mathrm{SU}(2)_{\theta=\pi} \mathrm{YM}$, in order to realize all global symmetries locally, necessarily couple to $5 \mathrm{~d}$ higher symmetry-protected topological states (SPTs, as invertible TQFTs [iTQFTs], as 5d one-form-centersymmetry-protected interacting "topological superconductors" in condensed matter).

We explored various $4 d$ Yang-Mills gauge theories living as boundary conditions of $5 \mathrm{~d}$ gapped short-/long-range entangled (SRE/LRE) topological states. We revisited $4 \mathrm{~d} \mathrm{SU}(2)_{\theta=\pi}$ YM-5d SRE-higher-SPTs coupled systems [5,8]. Follow 
(a)

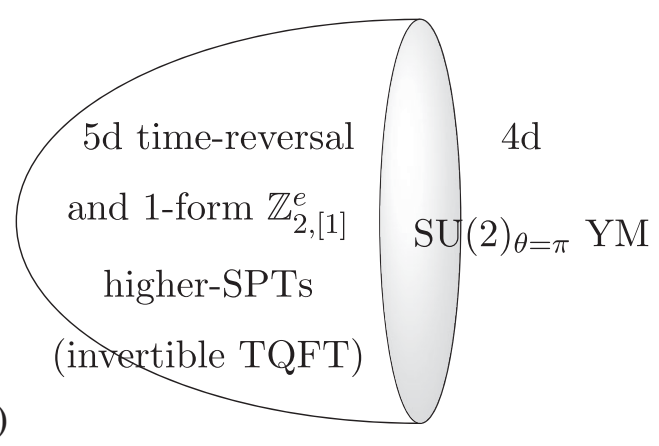

(b)

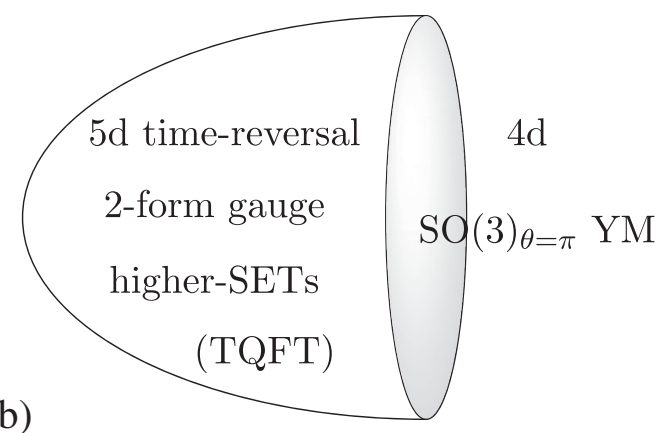

FIG. 16. An alternative illustration of Fig. 1: Various 4d Yang-Mills gauge theories live as the boundary conditions of certain 5d invertible TQFT (iTQFT) or 5d TQFT, in order to realize YM's (higher) global symmetries locally.

Weyl's gauge principle, by dynamically gauging the one-form center symmetry, we transformed a $5 \mathrm{~d}$ bulk SRE SPTs into an LRE symmetry-enriched topologically ordered state (SETs); thus we obtained the $4 \mathrm{~d} \mathrm{SO}(3)_{\theta=\pi}$ YM-5d LRE-higher-SETs coupled system with dynamical higher-form gauge fields. We illustrate such $4 \mathrm{~d}-5 \mathrm{~d}$ systems schematically in Fig. 1 and Fig. 16.

The $4 \mathrm{~d} \mathrm{SO}(3) \mathrm{YM}$ has a $\theta$ periodicity $\theta \sim \theta+$ $4 \pi$ on a spin manifold, and $\theta \sim \theta+8 \pi$ on a nonspin manifold. Since time-reversal symmetry is preserved if and only if $\theta \rightarrow-\theta$ is identified, thus $\operatorname{SO}(3)_{\theta=\pi}$ YM explicitly breaks the timereversal symmetry. In the right-hand side (b) of Fig. 1 and Fig. 16, we actually have a 5d SETs whose $4 \mathrm{~d}$ boundary explicitly breaks time-reversal symmetry.

Applying the tool introduced in [12], we derive new exotic anyonic statistics of extended objects such as two world sheet of strings and three world volume of branes, which physically characterize the 5d SETs. We discover new triple and quadruple link invariants associated with the underlying $5 \mathrm{~d}$ higher-gauge TQFT, hinting at a new intrinsic relation between nonsupersymmetric $4 \mathrm{~d}$ pure $\mathrm{YM}$ and topological links in 5d.

(2) Appearances of mod 2 anomalies.-We note that the anomaly associated with the $5 \mathrm{~d}$ term $\exp \left(\mathrm{i} \pi \int w_{3}(T M) B\right)$ has also appeared in the context of an adjoint $\mathrm{QCD}_{4}$ theory [55,86-88]. The $\exp \left(\mathrm{i} \pi \int w_{2}(T M) w_{3}(T M)\right)$ has also appeared as a new SU(2) anomaly in the SU(2) gauge theory [57]. All these anomalies and all our anomalies in Eq. (2.61) are mod 2 nonperturbative global anomalies, such as the $\mathrm{SU}(2)$ anomalies [56,57].

(3) Mathematical relation between $5 d$ and $4 d$ bordism groups.-Mathematically there seems to be an amusing relation between (1) gauging the SU(2) gauge bundle/connection under the coupling of $4 \mathrm{~d}$ YM with 4d SPTs (4d bordism invariants of $\Omega_{4}^{G^{\prime}}$ ) with $G^{\prime}$ derived from a group extension Eq. (3.2),

$$
1 \rightarrow \mathrm{SU}(2) \rightarrow G^{\prime} \rightarrow \mathrm{O}(d) \rightarrow 1
$$

and (2) some of the $5 \mathrm{~d}$ bordism invariants given by $\Omega_{5}^{\mathrm{O}}\left(\mathrm{B}^{2} \mathbb{Z}_{2}\right)=\mathbb{Z}_{2}^{4}$. We provide the computations of bordism groups and topological invariants in Appendix. It will be illuminating to explore this relation further in the future.

(4) Classes of $4 d \mathrm{SU}(2)_{\theta=\pi} Y M$.- - In Ref. [38], it was noted that the $\mathrm{Pin}^{+}$and $\mathrm{Pin}^{-}$versions of the above group extensions $G^{\prime}=\operatorname{Pin}^{+} \times_{\mathbb{Z}_{2}} \mathrm{SU}(2)$ and $G^{\prime}=$ $\mathrm{Pin}^{-} \times_{\mathbb{Z}_{2}} \mathrm{SU}(2)$ provide two different SPTs vacua after dynamically gauging the $\mathrm{SU}(2)$ symmetry to give rise to two distinct $4 \mathrm{~d} \mathrm{SU}(2)_{\theta=\pi} \mathrm{YM}$ theories. Although Ref. [38] suggested that the $\mathrm{Pin}^{+}$and $\mathrm{Pin}^{-}$ of $4 \mathrm{~d} \mathrm{SU}(2)_{\theta=\pi} \mathrm{YM}$ are secretly indistinguishable by correlators of local operators on orientable spacetimes or by gapped SPT states, they can be distinguished on nonorientable spacetimes or potentially by correlators of extended operators.

In this work, we have shown that $\mathrm{Pin}^{+}$and $\mathrm{Pin}^{-}$of $4 \mathrm{~d} \mathrm{SU}(2)_{\theta=\pi}$ YM indeed have distinct new higher 't Hooft anomalies, given by Eq. (2.38) and Eq. (2.61), with $\left(K_{1}, K_{2}\right)=(0,1)$ and $\left(K_{1}, K_{2}\right)=(1,1)$, respectively. Thus we confirm that $\mathrm{Pin}^{+}$and $\mathrm{Pin}^{-}$of $4 \mathrm{~d} \mathrm{SU}(2)_{\theta=\pi}$ YM live in distinct Hilbert spaces, and thus they are indeed distinct vacua.

More generally, in this work, we propose a classification $4 \mathrm{~d} \mathrm{SU}(2)_{\theta=\pi} \mathrm{YM}$ with a bosonic UV completion (e.g., on a lattice with bosonic degrees of freedom) and without fermionic parity symmetry $\mathbb{Z}_{2}^{F}$. We propose that a classification can all be obtained from dynamically gauging the $\mathrm{SU}(2)$ normal subgroup of $4 \mathrm{~d} G^{\prime}$ SPTs where $G^{\prime}$ is given by Eq. (3.2): $1 \rightarrow \mathrm{SU}(2) \rightarrow G^{\prime} \rightarrow \mathrm{O}(d) \rightarrow 1$; i.e., follow [38] by coupling the $4 \mathrm{~d} G^{\prime}$ SPTs to a pure $4 \mathrm{~d}$ $\mathrm{SU}(2) \mathrm{YM}$ theory and dynamically gauge their SU(2) (see Sec. III). ${ }^{46}$ Other than the Pin $^{+}$and

\footnotetext{
${ }^{46}$ Although we mainly focus on $4 \mathrm{~d} \mathrm{SU}(2)_{\theta=\pi}$ YM here, this gauge principle works for more general $4 \mathrm{~d} \mathrm{SU}(2) \mathrm{YM}$, or equivalently $4 \mathrm{~d}$ $\mathrm{SU}(2)$-gauge quantum spin liquids in condensed matter.
} 
Pin $^{-}$cases, this extension Eq. (3.2) includes two more cases: $G^{\prime}=\mathrm{O}(d) \times \mathrm{SU}(2)$ and $\mathrm{E}(d) \times_{\mathbb{Z}_{2}} \mathrm{SU}(2)$. In summary, in terms of the ungauged $4 \mathrm{~d}$ SPTs, we have the symmetry group $G^{\prime}[38,41]$ :

$$
G^{\prime}=\left\{\begin{array}{l}
\mathrm{O}(d) \times \mathrm{SU}(2)\left[\text { bosonic, relates to }\left(K_{1}, K_{2}\right)=(0,0)\right], \\
\mathrm{E}(d) \times_{\mathbb{Z}_{2}} \mathrm{SU}(2)\left[\text { bosonic, relates to }\left(K_{1}, K_{2}\right)=(1,0)\right], \\
\operatorname{Pin}^{+} \times_{\mathbb{Z}_{2}^{F}} \mathrm{SU}(2)\left[\text { fermionic, relates to }\left(K_{1}, K_{2}\right)=(0,1)\right], \\
\operatorname{Pin}^{-} \times_{\mathbb{Z}_{2}^{F}} \mathrm{SU}(2)\left[\text { fermionic, relatesto }\left(K_{1}, K_{2}\right)=(1,1)\right] .
\end{array}\right.
$$

The global symmetries of SPTs for the first two cases are purely bosonic since they do not contain $\mathbb{Z}_{2}^{F}$; the later two cases are fermionic since they do contain $\mathbb{Z}_{2}^{F}$ [the $\mathbb{Z}_{2}^{F}$ is shared by Pin and by the center of SU(2)]:

(a) Before gauging (see Fig. 17), the 4d SPTs are well-defined $4 \mathrm{~d}$ topological terms/bordism invariants that can live on $4 \mathrm{~d}$ with fully local on-site symmetry without the need of an extra $5 \mathrm{~d}$ bulk.

(b) After gauging the SU(2) normal subgroup of $G^{\prime}$ symmetry of these SPTs [see Fig. 16(a)], there is an emergent one-form center $\mathbb{Z}_{2,[1]}^{e}$ symmetry. After gauging $\mathrm{SU}(2)$, all four systems become bosonic without $\mathbb{Z}_{2}^{F}$ symmetry. We obtain new theories: $4 \mathrm{~d} \mathrm{SU}(2)_{\theta=\pi} \mathrm{YM}$ as boundary conditions of $5 \mathrm{~d}$ iTQFT in order to realize all global symmetries locally.

(c) Furthermore, after gauging this $\mathbb{Z}_{2,[1]}^{e}$ symmetry of the $4 \mathrm{~d} \mathrm{SU}(2)_{\theta=\pi}$ YM and 5d iTQFT coupled system [see Fig. 16(b)], we obtain new theories: $4 \mathrm{~d} \mathrm{SO}(3)_{\theta=\pi} \mathrm{YM}$ as boundary conditions of $5 \mathrm{~d}$ TQFT.

(5) Quantum spin liquids in condensed matter.-Strong coupled gauge theories have condensed matter

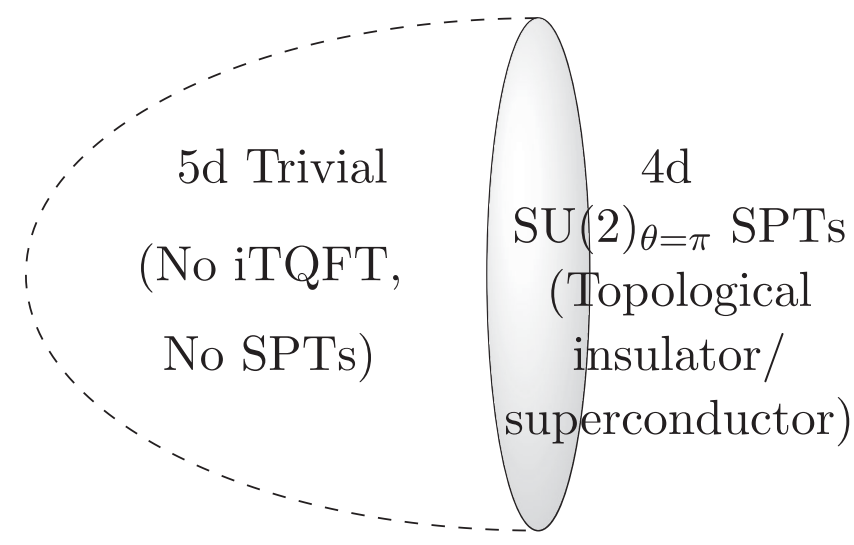

FIG. 17. The 4d time-reversal and SU(2) symmetric-protected topological states (SPTs) can be defined as 4d iTQFTs/bordism invariants. Their symmetries can be realized locally in $4 \mathrm{~d}$, without the need to an extra dimensional $5 d$ system. Gauging $\mathrm{SU}(2)$ symmetry of this $4 d$ SPTs renders the $4 d-5 d$ coupled system in Fig. 16(a). Further gauging $\mathbb{Z}_{2,[1]}^{e}$ symmetry of the whole system in Fig. 16(a) renders the 4d--5d coupled system in Fig. 16(b). See remark 4 of Sec. 9 for details. implications as quantum spin liquids. Time-reversal symmetric $\mathrm{U}(1)$ gauge theories as quantum spin liquids [30] have been explored and classified based on the quantum numbers of gapped electric and magnetic excitations (Wilson and 't Hooft line operators) in Refs. [63,64]; see also recent works $[89,90]$. Time-reversal symmetric $\mathrm{SU}(\mathrm{N})$ gauge theories as quantum spin liquids have been explored in [38]. We will leave additional interpretations of our results of non-Abelian $\mathrm{SU}(2)$ gauge theories in the context of quantum spin liquids for a future work.

(6) Relations of link invariants and braiding statistics in various dimensions. - We have applied the tools developed in [12] to compute link invariants of $5 \mathrm{~d}$ higher-gauge TQFTs in Sec. IV. We anticipate a precise mathematical formulation of this $5 \mathrm{~d}$ higher-gauge TQFT requires a certain higher category theory. We remark that several link invariants that we find here in $5 \mathrm{~d}$ have dimensional reduction analogy to $4 \mathrm{~d}$ and $3 \mathrm{~d}$, such that the dimensional reduced link configurations in $4 \mathrm{~d}$ and $3 \mathrm{~d}$ are related to what had been studied in $[11,12]$ and references therein.

(7) Fate of IR dynamics of gauge theories, UV completion, and lattice regularizations at $\theta=\pi$.-For the $4 \mathrm{~d}-5 \mathrm{~d}$ systems that we explore (schematically in Fig. 1 and Fig. 16), we mainly focus on their four siblings as the UV theories. We do not yet know the IR fate of their dynamics of these strongly coupled gauge theories given at UV. However, given the potentially complete' $t$ Hooft anomalies in Eq. (2.38) and Eq. (2.61) (at zero temperature), we can constrain the IR dynamics by UVIR anomaly matching. The consequence of anomaly matching implies that the IR theories must be at least one of the following scenarios:

(i) Time-reversal $\mathbb{Z}_{2}^{T}$ symmetry broken (spontaneously or explicitly): The conventional standard lore suggests the twofold $\mathbb{Z}_{2}^{T}$-breaking degenerate ground states [5].

(ii) One-form center $\mathbb{Z}_{2,[1]}^{e}$ symmetry broken (spontaneously or explicitly) as deconfinement:

(2-i) One-form $\mathbb{Z}_{2,[1]}^{e}$-breaking and deconfined TQFTs, i.e., topological order in condensed matter. $A \mathbb{Z}_{2}$ gauge theory with the one-form symmetry spontaneously broken has been proposed in Sec. VIII D. 
(2-ii) One-form $\mathbb{Z}_{2,[1]}^{e}$-breaking and deconfined gapless theories or deconfined CFTs.

(iii) One-form symmetry unbroken as confinement:

(3-i) One-form symmetry-extended invertible TQFT: This exotic scenario is discussed in Sec. VIII C. In Sec. VIII C, one-form $\mathbb{Z}_{2,[1]}$ symmetry is extended to $\mathbb{Z}_{4,[1]}$ in order to trivialize, and thus saturate, the anomaly.

(3-ii) One-form symmetry-preserving TQFT: Cordova and Ohmori $[84,85]$ have proved the nonexistence of TQFTs preserving both the one-form symmetry and timereversal symmetry while saturating the $4 \mathrm{~d}$ $\mathrm{SU}(\mathrm{N})_{\theta=\pi}$ YM's anomaly. This is consistent with Sec. VIII D's analysis, which results in the previous phase of (2-i).

(iv) Full symmetry-preserving gapless theory

(CFT): This is a fairly exotic case which seems to be less likely to happen.

Let us comment more on the recent Cordova-Ohmori's result $[84,85]$ which rules out the one-form and time-reversal symmetry-preserving gapped phases for $4 \mathrm{~d} \mathrm{SU}(\mathrm{N})_{\theta=\pi} \mathrm{YM}$, namely the phase of (3-ii). To recall, although in Sec. VIII C, we show the phase of (3-i) one-form symmetry-extended invertible TQFT can be constructed, but in reality, such phases are unlikely to happen [54] and should be only regarded as an intermediate step to construct the phase of (3-ii) one-form symmetry-preserving TQFT. Furthermore, we show in Sec. VIII D that dynamically gauging the extended symmetry of symmetry-extended invertible TQFT (of Sec. VIII C) results in the spontaneous symmetry-breaking phases, instead of the symmetry-preserving gapped phases. Thus our Sec. VIII is consistent with $[84,85]$.
The fate of any of the four scenarios of IR phases above is meant to match the anomaly (or match the Lieb-SchultzMattis-type of theorem in condensed matter physics). Since we have the four siblings of $4 \mathrm{~d} \mathrm{SU}(2)_{\theta=\pi} \mathrm{YM}$ at UV, labeled by $\left(K_{1}, K_{2}\right) \in\left(\mathbb{Z}_{2}, \mathbb{Z}_{2}\right)$, we can discuss each of their IR dynamics. We leave more systematic discussions of the IR dynamics for a future work.

(a) $4 \mathrm{~d} \mathrm{SU}(2)_{\theta=\pi} \mathrm{YM}$ with $\left(K_{1}, K_{2}\right)=(0,0)$ and $(1,0)$ : For $\left(K_{1}, K_{2}\right)=(0,0)$ or $(1,0)$ with $K_{2}=0$, we see that $\int_{M^{5}} B \mathrm{Sq}^{1} B+\mathrm{Sq}^{2} \mathrm{Sq}^{1} B+K_{1} w_{1}(T M)^{2} \times$ $\mathrm{Sq}^{1} B=\int_{M^{5}} \frac{1}{2} \tilde{w}_{1}(T M) \mathcal{P}(B)+K_{1} w_{1}(T M)^{2} \mathrm{Sq}^{1} B$ (or schematically $\sim \int_{M^{5}} \mathcal{T} B B+K_{1} \mathcal{T}^{3} B$ ) vanishes on $5 \mathrm{~d}$ orientable manifolds. In other words, if $\mathbb{Z}_{2}^{T}$ symmetry is spontaneously or explicitly broken, we can match the anomaly at IR. This means that when $K_{2}=0$, the $\mathrm{O}(d) \times \mathrm{SU}(2)$ and $\mathrm{E}(d) \times_{\mathbb{Z}_{2}} \mathrm{SU}(2)$ versions of $4 \mathrm{~d} \mathrm{SU}(2)_{\theta=\pi} \mathrm{YM}$ in Eq. (9.1) can indeed flow to the $\mathbb{Z}_{2}^{T}$-breaking scenario (1) at IR.

(b) $4 \mathrm{~d} \mathrm{SU}(2)_{\theta=\pi} \mathrm{YM}$ with $\left(K_{1}, K_{2}\right)=(0,1)$ and $(1,1)$ : For $\left(K_{1}, K_{2}\right)=(0,1)$ or $(1,1)$ with $K_{2}=1$, we see that $K_{2} \int_{M^{5}} \mathrm{Sq}^{1}\left(w_{2}(T M) B\right)$ is nonzero if $M^{5}$ has a boundary, and this $\int_{M^{5}} \mathrm{Sq}^{1}\left(w_{2}(T M) B\right)$ term does not vanish even if we restrict to orientable manifolds. In other words, even if we break $\mathbb{Z}_{2}^{T}$ symmetry (spontaneously or explicitly), the $\int \mathrm{Sq}^{1}\left(w_{2}(T M) B\right)$ still can suffer from background gauge variance. In this case, we should define the physical $4 \mathrm{~d}$ theory to include not only $\mathbf{Z}_{\mathrm{SU}(2)_{\theta=\pi} \mathrm{YM}}^{4 \mathrm{~d}}\left[M^{4} ; B, w_{j}(T M)\right]$ given in Eq. (2.37) but also the $4 \mathrm{~d}$ counterterm $\int \mathrm{Sq}^{1}\left(w_{2}(T M) B\right)$, which combine to

$$
\underset{\substack{\mathrm{SU}(2)_{\theta=n} \mathrm{YM} \\ \text { +counterterms }}}{4 \mathrm{~d}}\left[M^{4} ; B, w_{j}(T M)\right] \equiv \mathbf{Z}_{\mathrm{SU}(2)_{\theta=\pi} \mathrm{YM}}^{4 \mathrm{~d}}\left[M^{4} ; B, w_{j}(T M)\right] \cdot \exp \left(\mathrm{i} \pi \int K_{2} \mathrm{Sq}^{1}\left(w_{2}(T M) B\right)\right) .
$$

The intrinsic $5 \mathrm{~d}$ theory thus contains only the $5 \mathrm{~d}$ bordism invariant/SPTs/iTQFT, ${ }^{47}$

$$
\mathbf{Z}_{\mathrm{SPTs}}^{5 \mathrm{~d}}\left[M^{5} ; B, w_{j}(T M)\right]=\exp \left(\mathrm{i} \pi \int_{M^{5}} B \mathrm{Sq}^{1} B+\mathrm{Sq}^{2} \mathrm{Sq}^{1} B+K_{1} w_{1}(T M)^{2} \mathrm{Sq}^{1} B\right) .
$$

In fact, in Sec. VIII, we construct the 4d boundary based on the scenario (3) above as a boundary TQFT with a lattice spacetime path integral or a lattice Hamiltonian regularization; in this case, the full spacetime partition function $\mathbf{Z}[M]$ of the $4 \mathrm{~d}-5 \mathrm{~d}$ system can be explicitly computed as a number (by following Sec. 9 of [54]).

\footnotetext{
${ }^{47}$ Alternatively, if we instead interpret the background gauge variance of $\int \mathrm{Sq}^{1}\left(w_{2}(T M) B\right)$ as a $4 \mathrm{~d}$ higher 't Hooft anomaly (rather than just a $4 \mathrm{~d}$ counterterm), then it has a consequence on $4 \mathrm{~d} \mathrm{SU}(2)_{\theta=\pi} \mathrm{YM}$ dynamics. This means that when $K_{2}=1$, the $\operatorname{Pin}^{+}(d) \times$ $\mathrm{SU}(2)$ and $\mathrm{Pin}^{-}(d) \times_{\mathbb{Z}_{2}} \mathrm{SU}(2)$ versions of $4 \mathrm{~d} \mathrm{SU}(2)_{\theta=\pi} \mathrm{YM}$ in Eq. (9.1) cannot flow merely to the $\mathbb{Z}_{2}^{T}$-breaking scenario (1) at IR. However, scenarios (2), (3), and (4) are still possible IR fates. It seems that scenario (2) with $\mathbb{Z}_{2,[1]}^{e}$ breaking with deconfinement (due to the perimeter law of the Wilson loop) can be the most likely outcome. Scenario (2-ii) for $\operatorname{Pin}^{+}(d) \times \mathrm{SU}(2)$ and $\operatorname{Pin}^{-}(d) \times_{\mathbb{Z}_{2}} \mathrm{SU}(2)$ versions of $4 \mathrm{~d} \mathrm{SU}(2)_{\theta=\pi} \mathrm{YM}$ are proposed in [38] as two distinct versions of deconfined gapless theories or deconfined CFTs: In this work, we show that these two siblings are indeed distinct theories with different Hilbert spaces at UV, due to their distinct $4 \mathrm{~d}$ anomalies differed by a $5 \mathrm{~d}$ invariant $\int_{M^{5}} K_{1} w_{1}(T M)^{2} \mathrm{Sq}^{1} B \sim \int_{M^{5}} K_{1} \mathcal{T}^{3} B$. However, we will see that interpreting $\int_{M^{5}} \mathrm{Sq}^{1}\left(w_{2}(T M) B\right)$ as a $4 \mathrm{~d}$ higher 't Hooft anomaly will lead to a rather bizarre and strong constraint on the dynamics of $4 \mathrm{~d} S \mathrm{SU}(2)_{\theta=0} \mathrm{YM}$; see footnote 47.
} 
We will revisit other issues of dynamics in the future.

(8) Refinement and modification of Yang-Mills existence and mass gap problem at $\theta=0$. - The original statement of the Clay Math Millennium Prize Problem [3] is "Prove that for any compact simple gauge group $G$, a nontrivial quantum Yang-Mills theory exists on $\mathbb{R}^{4}$ and has a mass gap $\Delta>0$." It seems that in addition to the specification of gauge group $G$ and topological term $\frac{\theta}{8 \pi^{2}} \int_{M^{4}} \operatorname{Tr}(F \wedge F)$ with $\theta=0$, we may also need to specify the data $\left(K_{1}, K_{2}\right) \in$ $\left(\mathbb{Z}_{2}, \mathbb{Z}_{2}\right)$ for Kramers single/doublet and bosonic/ fermionic statistics for a quantum number of Wilson lines $W_{e}$, as we did in Eq. (2.25) and Eq. (2.37), say for $4 \mathrm{~d} \mathrm{SU}(2)_{\theta=0} \mathrm{YM}$. The data $\left(K_{1}, K_{2}\right) \in\left(\mathbb{Z}_{2}, \mathbb{Z}_{2}\right)$ may have been ignored in the past.

Here are possible outcomes for four siblings $\left(K_{1}, K_{2}\right) \in\left(\mathbb{Z}_{2}, \mathbb{Z}_{2}\right)$ of $4 \mathrm{~d} \mathrm{SU}(2)_{\theta=0}$ YM. Notice that the transition from the vacua of $4 \mathrm{~d} S \mathrm{~S}(2)_{\theta=\pi} \mathrm{YM}$ to $4 \mathrm{~d} \mathrm{SU}(2)_{\theta=0} \mathrm{YM}$ must break $\mathbb{Z}_{2}^{T}$ in between $0<\theta<\pi$.

(a) $4 \mathrm{~d} \mathrm{SU}(2)_{\theta=0} \mathrm{YM}\left(K_{1}, K_{2}\right)=(0,0)$ and $(1,0)$ : Since the anomalies associated with $4 \mathrm{~d}$ $\mathrm{SU}(2)_{\theta=\pi} \mathrm{YM}$ with $\left(K_{1}, K_{2}\right)=(0,0)$ and $(1,0)$ can be removed by $\mathbb{Z}_{2}^{T}$ breaking, therefore $4 \mathrm{~d} \mathrm{SU}(2)_{\theta=0} \mathrm{YM}\left(K_{1}, K_{2}\right)=(0,0)$ and $(1,0)$ can have no 't Hooft anomaly; thus it can be trivially gapped as a trivial vacuum.

(b) $4 \mathrm{~d} \mathrm{SU}(2)_{\theta=0} \mathrm{YM}$ with $\left(K_{1}, K_{2}\right)=(0,1)$ and $(1,1)$ : These two siblings only differ from the trivially gapped vacuum of the previous two siblings, $\left(K_{1}, K_{2}\right)=(0,0)$ and $(1,0)$, by the $4 \mathrm{~d}$ counterterm $\int K_{2} \mathrm{Sq}^{1}\left(w_{2}(T M) B\right) .{ }^{48}$
It will be enlightening to contemplate more consequences of their IR dynamics for these four siblings $\left(K_{1}, K_{2}\right) \in\left(\mathbb{Z}_{2}, \mathbb{Z}_{2}\right)$ of $4 \mathrm{~d} \mathrm{SU}(2) \mathrm{YM}$.

\section{ACKNOWLEDGMENTS}

The authors are listed in alphabetical order by the standard convention. J. W. thanks Pierre Deligne, Juan Maldacena, Max Metlitski, Kantaro Ohmori, Nathan Seiberg, Edward Witten, and Shing-Tung Yau for encouraging conversations, thanks Robert Gompf and Pavel Putrov for research collaborations in Ref. [91] and in Refs. [12,38], respectively. We also thank Clay Cordova, Zohar Komargodski, and Ho Tat Lam for conversations. J. W. thanks Harvard CMSA for the seminar invitation on presenting this specific work [92] and thanks the audience for the feedback at the IAS Princeton seminar [93]. Z. W. acknowledges support from NSFC Grants No. 11431010 and No. 11571329. Z. W. is supported by the Shuimu Tsinghua Scholar Program. J. W. is supported by NSF Grant No. PHY-1606531. Y. Z. thanks the Physics Department of Princeton University for support. This work is also supported by NSF Grant No. DMS-1607871 "Analysis, Geometry and Mathematical Physics" and Center for Mathematical Sciences and Applications at Harvard University.

\section{APPENDIX: COMPUTATION OF BORDISM GROUPS}

In this appendix, we compute the bordism groups $\Omega_{d}^{G^{\prime}}$ where $G^{\prime}$ is a solution of all possible extensions of

$$
1 \rightarrow \mathrm{SU}(2) \rightarrow G^{\prime} \rightarrow \mathrm{O}(d) \rightarrow 1
$$

given by Eq. (9.1)

$$
G^{\prime}=\left\{\begin{array}{l}
\mathrm{O}(d) \times \mathrm{SU}(2)\left[\text { bosonic, relates to }\left(K_{1}, K_{2}\right)=(0,0)\right. \text { in Appendix A.1, } \\
\mathrm{E}(d) \times_{\mathbb{Z}_{2}} \mathrm{SU}(2)\left[\text { bosonic, relates to }\left(K_{1}, K_{2}\right)=(1,0)\right] \text { in Appendix A.2, } \\
\mathrm{Pin}^{+} \times_{\mathbb{Z}_{2}^{F}} \mathrm{SU}(2)\left[\text { fermionic, relates to }\left(K_{1}, K_{2}\right)=(0,1)\right] \text { in Appendix A.3 } \\
\mathrm{Pin}^{-} \times_{\mathbb{Z}_{2}^{F}} \mathrm{SU}(2)\left[\text { fermionic, relates to }\left(K_{1}, K_{2}\right)=(1,1)\right] \text { in Appendix A.4. }
\end{array}\right.
$$

The bordism groups and their bordism invariants (topological invariants and SPTs) are used in the main text, for example, $\Omega_{d=4}^{G^{\prime}}$ in Sec. III. We also compute $\Omega_{d=5}^{\mathrm{O}}\left(\mathrm{B}^{2} \mathbb{Z}_{2}\right)$, used in Sec. II, in Appendix A 5.

\footnotetext{
${ }^{48}$ Follow the earlier footnote 46: if we instead interpret the background gauge variance of $\int \operatorname{Sq}^{1}\left(w_{2}(T M) B\right)$ as a $4 \mathrm{~d}$ higher 't Hooft anomaly (rather than just a $4 \mathrm{~d}$ counterterm), then it has a consequence on $4 \mathrm{~d} \mathrm{SU}(2)_{\theta=0} \mathrm{YM}$ dynamics also. Notice that $\int \mathrm{Sq}^{1}\left(w_{2}(T M) B\right)$ survive without $\mathbb{Z}_{2}^{T}$ protection; therefore if there is an "anomaly" $\int \mathrm{Sq}^{1}\left(w_{2}(T M) B\right)$ at $\theta=\pi$, then it remains for all $0 \leq \theta \leq \pi$ including at $\theta=0$. The only way to saturate the if anomaly of $\int K_{2} \mathrm{Sq}^{1}\left(w_{2}(T M) B\right)$ for $\mathrm{SU}(2)_{\theta=0} \mathrm{YM}$ is breaking the one-form symmetry. If so, this means that $\mathrm{SU}(2)_{\theta=0} \mathrm{YM}$ with the fermionic Wilson line (i.e., $K_{2}=1$ ) has one-form symmetry spontaneously broken thus deconfined, which cannot be trivially gapped nor a trivial vacuum. In this case, if $4 \mathrm{~d} \mathrm{SU}(2)_{\theta=0} \mathrm{YM}$ with $K_{2}=1$ is still gapped as the conventional wisdom goes, they belong to the scenarios:

(i) (2-i) One-form $\mathbb{Z}_{2,[1]}^{e}$-breaking and deconfined TQFTs, i.e., topological order.

This deconfined scenario seems to be too exotic for $\mathrm{SU}(2)_{\theta=0} \mathrm{YM}$ merely with the fermionic Wilson line. Therefore, our canonical interpretation with $\int \mathrm{Sq}^{1}\left(w_{2}(T M) B\right)$ being a $4 \mathrm{~d}$ counterterm in $4 \mathrm{~d} \mathrm{YM}$ [see the main text around Eq. (9.2)] avoids leading to this bizarre deconfinement scenario for $\mathrm{SU}(2)_{\theta=0} \mathrm{YM}$.
} 
In the two subsections Appendix A 3 and A 4, we will encounter the $\mathcal{A}_{2}(1)$ module structure due to the appearance of $M$ Spin in the decomposition of $M T\left(\operatorname{Pin}^{ \pm} \times_{\mathbb{Z}_{2}} \mathrm{SU}(2)\right)$.

Readers can find the introduction to this computation in Refs. $[10,38,41]$. For a short summary of the concepts and terminology used here, the readers may consult a succinct summary in Appendix B of [38]. For readers who are not familiar with the details of mathematical calculations, we will help by stating the results explicitly.

\section{Bordism group of $\mathrm{O} \times \mathrm{SU}(2): \Omega_{d}^{\mathrm{O} \times \mathrm{SU}(2)}$}

We first notice that $M T(\mathrm{O} \times \mathrm{SU}(2))=M \mathrm{O} \wedge \mathrm{BSU}(2)_{+}$, where $\wedge$ is the smash product and $\mathrm{SU}(2)_{+}$is the disjoint union of the topological space $\mathrm{SU}(2)$ and a point. $M T H$ is the Madsen-Tillmann spectrum of the group $H$, and $M H$ is the Thom spectrum of the group $H$. By the Adams spectral sequence, we have

$$
\operatorname{Ext}_{\mathcal{A}_{2}}^{s, t}\left(\mathrm{H}^{*}\left(M \mathrm{O} \wedge \mathrm{BSU}(2)_{+}, \mathbb{Z}_{2}\right), \mathbb{Z}_{2}\right) \Rightarrow \Omega_{t-s}^{\mathrm{O} \times \mathrm{SU}(2)} .
$$

The mod 2 cohomology of Thom spectrum $M O$ is

$$
\mathrm{H}^{*}\left(M \mathrm{O}, \mathbb{Z}_{2}\right)=\mathcal{A}_{2} \otimes \Omega^{*}
$$

where $\Omega=\mathbb{Z}_{2}\left[y_{2}, y_{4}, y_{5}, y_{6}, y_{8}, \ldots\right]$ is the unoriented bordism ring and $\Omega^{*}$ is the $\mathbb{Z}_{2}$-linear dual of $\Omega$. Here $y_{i}$ are manifold generators; for example, $y_{2}=\mathbb{R P}^{2}, y_{4}=\mathbb{R P}^{4}$, and $y_{5}$ is $\mathrm{Wu}$ manifold $\mathrm{SU}(3) / \mathrm{SO}(3)$.

On the other hand, $H^{*}\left(M \mathrm{O}, \mathbb{Z}_{2}\right)=\mathbb{Z}_{2}\left[w_{1}, w_{2}, w_{3}, \ldots\right] U$, where $U$ is the Thom class of the virtual bundle (of dimension 0) over BO which is the colimit of $E_{n}-n$. Here $E_{n}$ is the universal $n$ bundle over $\mathrm{BO}(n)$, and $w_{i}$ is the $i$ th Stiefel-Whitney class of the virtual bundle (of dimension 0 ) over BO. Note that the pullback of the virtual bundle (of dimension 0) over $\mathrm{BO}$ along the map $g: M \rightarrow \mathrm{BO}$ is just $T M-d$ where $M$ is a $d$-dimensional manifold, $T M$ is the tangent bundle of $M$, and $g$ is given by the $\mathrm{O}$ structure on $M$. We will not distinguish $w_{i}$ and $w_{i}(T M)$.

By Thom's result, two manifolds are unorientedly bordant if and only if they have identical sets of Stiefel-Whitney characteristic numbers. The nonvanishing Stiefel-Whitney numbers of $y_{2}=\mathbb{R} \mathbb{P}^{2}$ are $w_{2}$ and $w_{1}^{2}$, the nonvanishing Stiefel-Whitney numbers of $y_{2}^{2}=\mathbb{R} \mathbb{P}^{2} \times \mathbb{R} \mathbb{P}^{2}$ are $w_{2}^{2}$ and $w_{4}$, the nonvanishing Stiefel-Whitney numbers of $y_{4}=\mathbb{R P}^{4}$ are $w_{1}^{4}$ and $w_{4}$, and the only nonvanishing Stiefel-Whitney number of $\mathrm{Wu}$ manifold $\mathrm{SU}(3) / \mathrm{SO}(3)$ is $w_{2} w_{3}$. So $y_{2}^{*}=w_{1}^{2}$ or $w_{2},\left(y_{2}^{2}\right)^{*}=w_{2}^{2}, y_{4}^{*}=w_{1}^{4}, y_{5}^{*}=w_{2} w_{3}$, etc., where $y_{i}^{*}$ is the $\mathbb{Z}_{2}$-linear dual of $y_{i} \in \Omega$. Below we choose $y_{2}^{*}=w_{1}^{2}$ by default, and this is reasonable since $\operatorname{Sq}^{2}\left(x_{d-2}\right)=$ $\left(w_{2}+w_{1}^{2}\right) x_{d-2}$ on the $d$ manifold by the Wu formula. Since

$$
H^{*}\left(\operatorname{BSU}(2), \mathbb{Z}_{2}\right)=\mathbb{Z}_{2}\left[c_{2}\right],
$$

by the Künneth formula, we get

$$
\begin{aligned}
\mathrm{H}^{*} & \left(M \mathrm{O} \wedge \mathrm{BSU}(2)_{+}, \mathbb{Z}_{2}\right) \\
& =\mathrm{H}^{*}\left(M \mathrm{O}, \mathbb{Z}_{2}\right) \otimes \mathrm{H}^{*}\left(\mathrm{BSU}(2), \mathbb{Z}_{2}\right) \\
& =\mathcal{A}_{2} \oplus \Sigma^{2} \mathcal{A}_{2} \oplus 3 \Sigma^{4} \mathcal{A}_{2} \oplus \Sigma^{5} \mathcal{A}_{2} \oplus \cdots
\end{aligned}
$$

Here $\Sigma^{n} \mathcal{A}_{2}$ is the $n$th iterated shift of the graded algebra $\mathcal{A}_{2}$. In summary we have $\Omega_{4}^{\mathrm{O} \times \mathrm{SU}(2)}=\mathbb{Z}_{2}^{3}, \Omega_{5}^{\mathrm{O} \times \mathrm{SU}(2)}=\mathbb{Z}_{2}$.

The bordism invariants of $\Omega_{4}^{\mathrm{O} \times \mathrm{SU}(2)}=\mathbb{Z}_{2}^{3}$ are $w_{1}^{4}, w_{2}^{2}$, and $c_{2} \bmod 2$. Namely, in physics terms, the topological invariants/SPTs from $\Omega_{4}^{\mathrm{O} \times \mathrm{SU}(2)}$ are $w_{1}(T M)^{4}, w_{2}(T M)^{2}$, and $c_{2} \bmod 2$.

The bordism invariant of $\Omega_{5}^{\mathrm{O} \times \mathrm{SU}(2)}=\mathbb{Z}_{2}$ is $w_{2} w_{3}$. Namely, in physics terms, the topological invariants/ SPTs from $\Omega_{5}^{\mathrm{O} \times \mathrm{SU}(2)}$ are $w_{2}(T M) w_{3}(T M)$.

\section{Bordism group of $\mathrm{E} \times_{\mathbb{Z}_{2}} \mathrm{SU}(2): \Omega_{d}^{\mathrm{E} \times_{\mathbb{Z}_{2}} \mathrm{SU}(2)}$}

From the short exact sequence

$$
1 \rightarrow \mathrm{SO} \rightarrow \mathrm{E} \rightarrow \mathbb{Z}_{4} \rightarrow 1
$$

we derive the short exact sequence

$$
1 \rightarrow \mathrm{SO} \rightarrow \mathrm{E} \times_{\mathbb{Z}_{2}} \mathrm{SU}(2) \rightarrow \mathbb{Z}_{4} \times_{\mathbb{Z}_{2}} \mathrm{SU}(2) \rightarrow 1 .
$$

Hence $M T\left(E \times_{\mathbb{Z}_{2}} \mathrm{SU}(2)\right)=M S O \wedge \Sigma^{-3} M T\left(\operatorname{Spin}(3) \times_{\mathbb{Z}_{2}}\right.$ $\left.\mathbb{Z}_{4}\right)=M S O \wedge \Sigma^{-3} M T \operatorname{Pin}^{+}(3)=M S O \wedge \Sigma^{-3} M \operatorname{Spin}(3) \wedge$ $\Sigma^{-2} M \mathbb{Z}_{2}=M \mathrm{O} \wedge \Sigma^{-4} M \operatorname{Spin}(3)$, where $\wedge$ is the smash product, $\Sigma$ is the suspension, MTH is the MadsenTillmann spectrum of the group $H$, and $M H$ is the Thom spectrum of the group $H$.

By the Adams spectral sequence,

$\operatorname{Ext}_{\mathcal{A}_{2}}^{s, t}\left(\mathrm{H}^{*}\left(M \mathrm{O} \wedge \Sigma^{-4} M \mathrm{SU}(2), \mathbb{Z}_{2}\right), \mathbb{Z}_{2}\right) \Rightarrow \Omega_{t-s}^{\mathrm{Ex} \mathbb{Z}_{2}} \mathrm{SU}(2)$

Since

$$
\mathrm{H}^{*}\left(\Sigma^{-4} M \mathrm{SU}(2), \mathbb{Z}_{2}\right)=\mathbb{Z}_{2}\left[c_{2}\right] U,
$$

where $U$ is the Thom class, by the Künneth formula,

$$
\begin{aligned}
\mathrm{H}^{*} & \left(M \mathrm{O} \wedge \Sigma^{-4} M S \mathrm{SU}(2), \mathbb{Z}_{2}\right) \\
& =\mathrm{H}^{*}\left(M \mathrm{O}, \mathbb{Z}_{2}\right) \otimes \mathrm{H}^{*}\left(\Sigma^{-4} M \mathrm{SU}(2), \mathbb{Z}_{2}\right) \\
& =\mathcal{A}_{2} \oplus \Sigma^{2} \mathcal{A}_{2} \oplus 3 \Sigma^{4} \mathcal{A}_{2} \oplus \Sigma^{5} \mathcal{A}_{2} \oplus \cdots
\end{aligned}
$$

Hence we have $\Omega_{4}^{\mathrm{E} \times_{\mathbb{Z}_{2}} \mathrm{SU}(2)}=\mathbb{Z}_{2}^{3}, \Omega_{5}^{\mathrm{E} \times_{\mathbb{Z}_{2}} \mathrm{SU}(2)}=\mathbb{Z}_{2}$.

The bordism invariants of $\Omega_{4}^{\mathrm{E} \times_{\mathbb{Z}_{2}} \mathrm{SU}(2)}=\mathbb{Z}_{2}^{3}$ are $w_{1}^{4}, w_{2}^{2}$, and $c_{2} \bmod 2$. Namely, in physics terms, the topological invariants/SPTs from $\Omega_{4}^{\mathrm{E}_{\mathbb{Z}_{2}} \mathrm{SU}(2)}$ are $w_{1}(T M)^{4}, w_{2}(T M)^{2}$, and $c_{2} \bmod 2$. Since the constraint $w_{1}(T M)^{2}=w_{2}\left(V_{\mathrm{SO}(3)}\right)$ 
is satisfied, let $\beta_{2}$ denote the Bockstein homomorphism associated with the extension $\mathbb{Z} \rightarrow \mathbb{Z} \rightarrow \mathbb{Z}_{2}$. Then $W_{3}\left(V_{\mathrm{SO}(3)}\right)=\beta_{2} w_{2}\left(V_{\mathrm{SO}(3)}\right)=\beta_{2} w_{1}(T M)^{2}=$

$\beta_{2} \mathrm{Sq}^{1} w_{1}(T M)=0$ where $W_{3}\left(V_{\mathrm{SO}(3)}\right)$ is the third integral Stiefel-Whitney class of $V_{\mathrm{SO}(3)}$ and we have used the fact that $\beta_{2} \mathrm{Sq}^{1}=0$. Hence $V_{\mathrm{SO}(3)}$ lifts to a $\operatorname{Spin}^{c}(3)=\mathrm{U}(2)$ bundle $V_{\mathrm{U}(2)}$, where $c_{2}=c_{2}\left(V_{\mathrm{U}(2)}\right)$ is the second Chern class of $V_{\mathrm{U}(2)}$.

The bordism invariants of $\Omega_{5}^{\mathrm{E} \times_{\mathbb{Z}_{2}} \mathrm{SU}(2)}$ are $w_{2} w_{3}$. Namely, in physics terms, the topological invariants/SPTs from $\Omega_{5}^{\mathrm{E \times} \overline{\mathbb{Z}}_{2} \mathrm{SU}(2)}$ are $w_{2}(T M) w_{3}(T M)$.

\section{Bordism group of $\operatorname{Pin}^{+} x_{\mathbb{Z}_{2}} \mathrm{SU}(2): \Omega_{d}^{\mathrm{Pin}^{+}{ }_{\mathbb{Z}_{2}} \mathrm{SU}(2)}$}

Since there is a homotopy pullback square

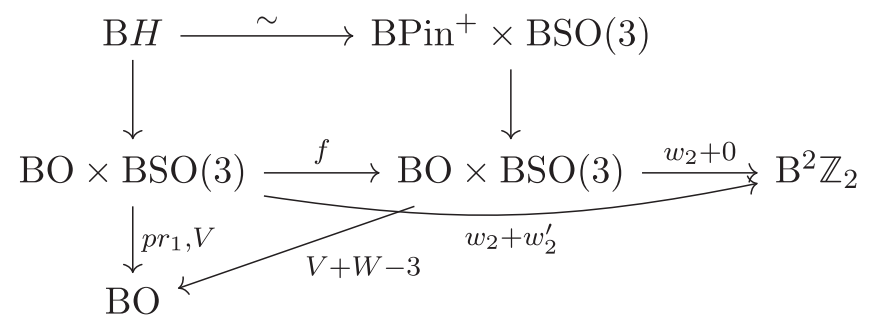

where $f$ maps $(V, W)$ to $(V-W+3, W)$, we have $M T H=M T$ Pin $^{+} \wedge \Sigma^{-3} M S O(3)=M S \operatorname{Spin} \wedge \Sigma^{-3} M \mathrm{O}(3)$. By the Adams spectral sequence,

$\operatorname{Ext}_{\mathcal{A}_{2}}^{s, t}\left(\mathrm{H}^{*}\left(M \operatorname{Spin} \wedge \Sigma^{-3} M \mathrm{O}(3), \mathbb{Z}_{2}\right), \mathbb{Z}_{2}\right) \Rightarrow \Omega_{t-s}^{\mathrm{Pin}^{+} x_{\mathbb{Z}_{2}} \mathrm{SU}(2)}$

The mod 2 cohomology of Thom spectrum MSpin is

$$
\mathrm{H}^{*}\left(M \text { Spin, } \mathbb{Z}_{2}\right)=\mathcal{A}_{2} \otimes_{\mathcal{A}_{2}(1)}\left\{\mathbb{Z}_{2} \oplus M\right\},
$$

where $M$ is a graded $\mathcal{A}_{2}(1)$ module with the degree $i$ homogeneous part $M_{i}=0$ for $i<8$. Here $\mathcal{A}_{2}(1)$ stands for the subalgebra of $\mathcal{A}_{2}$ generated by $\mathrm{Sq}^{1}$ and $\mathrm{Sq}^{2}$.

For $t-s<8$, we can identify the $E_{2}$ page with

$$
\operatorname{Ext}_{\mathcal{A}_{2}(1)}^{s, t}\left(\mathrm{H}^{*+3}\left(M \mathrm{O}(3), \mathbb{Z}_{2}\right), \mathbb{Z}_{2}\right) .
$$

For other details and the computation of the $\mathcal{A}_{2}(1)$ module structure and Adams chart, please consult Refs. [38,41]. We can extract the bordism group and their bordism invariants from [38].
4. Bordism group of $\mathrm{Pin}^{-} \times_{\mathbb{Z}_{2}} \mathrm{SU}(2): \Omega_{d}^{\mathrm{Pin}^{-} x_{\mathbb{Z}_{2}} \mathrm{SU}(2)}$

Since there is a homotopy pullback square

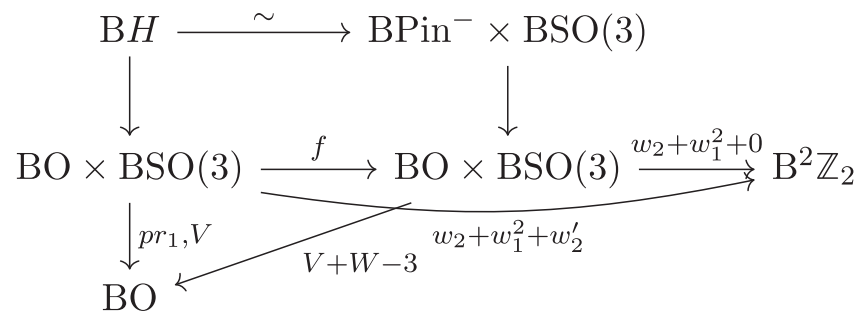

where $f$ maps $(V, W)$ to $(V-W+3, W)$, we have $M T H=M T \mathrm{Pin}^{-} \wedge \Sigma^{-3} M \mathrm{SO}(3)=M \operatorname{Spin} \wedge \Sigma^{3} M T \mathrm{O}(3)$. By the Adams spectral sequence,

$\operatorname{Ext}_{\mathcal{A}_{2}}^{s, t}\left(\mathrm{H}^{*}\left(M \operatorname{Spin} \wedge \Sigma^{3} M T \mathrm{O}(3), \mathbb{Z}_{2}\right), \mathbb{Z}_{2}\right) \Rightarrow \Omega_{t-s}^{\mathrm{Pin}^{-} \times_{\mathbb{Z}_{2}} \mathrm{SU}(2)}$.

For $t-s<8$, we can identify the $E_{2}$ page with

$$
\operatorname{Ext}_{\mathcal{A}_{2}(1)}^{s, t}\left(\mathrm{H}^{*-3}\left(M T \mathrm{O}(3), \mathbb{Z}_{2}\right), \mathbb{Z}_{2}\right)
$$

For other details and the computation of the $\mathcal{A}_{2}(1)$ module structure and Adams chart, please consult Refs. [38,41]. We can extract the bordism group and their bordism invariants from [38].

\section{Bordism group of $\mathrm{O} \times \mathbb{Z}_{2,[1]}^{e}: \Omega_{d}^{\mathrm{O}}\left(\mathrm{B}^{2} \mathbb{Z}_{2}\right)$}

By the Adams spectral sequence,

$\operatorname{Ext}_{\mathcal{A}_{2}}^{s, t}\left(\mathrm{H}^{*}\left(M \mathrm{O} \wedge\left(\mathrm{B}^{2} \mathbb{Z}_{2}\right)_{+}, \mathbb{Z}_{2}\right), \mathbb{Z}_{2}\right) \Rightarrow \Omega_{t-s}^{\mathrm{O}}\left(\mathrm{B}^{2} \mathbb{Z}_{2}\right)$.

Since $\mathrm{H}^{*}\left(\mathrm{~B}^{2} \mathbb{Z}_{2}, \mathbb{Z}_{2}\right)=\mathbb{Z}_{2}\left[x_{2}, x_{3}, x_{5}, x_{9}, \ldots\right]$ where $x_{2}$ is the generator of $\mathrm{H}^{2}\left(\mathrm{~B}^{2} \mathbb{Z}_{2}, \mathbb{Z}_{2}\right), x_{3}=\mathrm{Sq}^{1} x_{2}, x_{5}=\mathrm{Sq}^{2} x_{3}$, $x_{9}=\mathrm{Sq}^{4} x_{5}$, etc., so by the Künneth formula,

$$
\begin{aligned}
\mathrm{H}^{*} & \left(M \mathrm{O} \wedge\left(\mathrm{B}^{2} \mathbb{Z}_{2}\right)_{+}, \mathbb{Z}_{2}\right) \\
& =\mathrm{H}^{*}\left(M \mathrm{O}, \mathbb{Z}_{2}\right) \otimes \mathrm{H}^{*}\left(\mathrm{~B}^{2} \mathbb{Z}_{2}, \mathbb{Z}_{2}\right) \\
& =\mathcal{A}_{2} \otimes \mathbb{Z}_{2}\left[y_{2}, y_{4}, y_{5}, y_{6}, y_{8}, \ldots\right]^{*} \otimes \mathbb{Z}_{2}\left[x_{2}, x_{3}, x_{5}, x_{9}, \ldots\right] \\
& =\mathcal{A}_{2} \oplus 2 \Sigma^{2} \mathcal{A}_{2} \oplus \Sigma^{3} \mathcal{A}_{2} \oplus 4 \Sigma^{4} \mathcal{A}_{2} \oplus 4 \Sigma^{5} \mathcal{A}_{2} \oplus \cdots
\end{aligned}
$$

Hence we have $\Omega_{4}^{\mathrm{O}}\left(\mathrm{B}^{2} \mathbb{Z}_{2}\right)=\mathbb{Z}_{2}^{4}, \Omega_{5}^{\mathrm{O}}\left(\mathrm{B}^{2} \mathbb{Z}_{2}\right)=\mathbb{Z}_{2}^{4}$.

The bordism invariants of $\Omega_{4}^{\mathrm{O}}\left(\mathrm{B}^{2} \mathbb{Z}_{2}\right)=\mathbb{Z}_{2}^{4}$ are $x_{2}^{2}, w_{1}^{4}$, $w_{1}^{2} x_{2}$, and $w_{2}^{2}$. Namely, in physics terms, the topological invariants/SPTs from $\Omega_{4}^{\mathrm{O}}\left(\mathrm{B}^{2} \mathbb{Z}_{2}\right)$ are $B^{2}$, $w_{1}(T M)^{4}, w_{1}(T M)^{2} B$, and $w_{2}(T M)^{2}$. 
The bordism invariants of $\Omega_{5}^{\mathrm{O}}\left(\mathrm{B}^{2} \mathbb{Z}_{2}\right)=\mathbb{Z}_{2}^{4}$ are $x_{2} x_{3}, x_{5}$, $w_{1}^{2} x_{3}$, and $w_{2} w_{3}$. Namely, in physics terms, the topological invariants/SPTs from $\Omega_{5}^{\mathrm{O}}\left(\mathrm{B}^{2} \mathbb{Z}_{2}\right)$ are $B \mathrm{Sq}^{1} B, \mathrm{Sq}^{2} \mathrm{Sq}^{1} B$,
$w_{1}(T M)^{2} \mathrm{Sq}^{1} B$, and $w_{2}(T M) w_{3}(T M)$. Readers can find more detailed discussions and calculations of the cobordism theory of higher symmetries in Ref. [10].
[1] W. Pauli, Relativistic field theories of elementary particles, Rev. Mod. Phys. 13, 203 (1941).

[2] C. N. Yang and R. L. Mills, Conservation of isotopic spin and isotopic gauge invariance, Phys. Rev. 96, 191 (1954).

[3] A. Jaffe and E. Witten, Quantum Yang-Mills Theory, Clay Mathematics Institute Millennium Prize Problems (Clay Mathematics Institute, Cambridge, MA, 2004).

[4] G. 't Hooft, C. Itzykson, A. Jaffe, H. Lehmann, P. K. Mitter, I. M. Singer et al., Recent Developments in Gauge Theories., Proceedings Nato Advanced Study Institute, Cargese, France, 1979, NATO Science Series B, Vol. 59 (1980).

[5] D. Gaiotto, A. Kapustin, Z. Komargodski, and N. Seiberg, Theta, time reversal, and temperature, J. High Energy Phys. 05 (2017) 091.

[6] D. Gaiotto, A. Kapustin, N. Seiberg, and B. Willett, Generalized global symmetries, J. High Energy Phys. 02 (2015) 172.

[7] C. G. Callan, Jr. and J. A. Harvey, Anomalies and Fermion zero modes on strings and domain walls, Nucl. Phys. B250, 427 (1985).

[8] Z. Wan and J. Wang, New higher anomalies, SU(N) Yang-Mills gauge theory and $\mathbb{C P}^{\mathrm{N}-1}$ Sigma model, arXiv: 1812.11968

[9] Z. Wan, J. Wang, and Y. Zheng, Mixed Higher Anomalies under CPT Discrete Symmetries: Application to NonAbelian Gauge Theory and Sigma Model (to be published).

[10] Z. Wan and J. Wang, Non-Abelian gauge theories, Sigma models, and higher Cobordisms I: Classification of highersymmetry protected topological states and higher anomalies via a generalized Cobordism theory, Ann. Math. Sci. Appl. 4, 107 (2019).

[11] J. Wang, X.-G. Wen, and S.-T. Yau, Quantum statistics and spacetime surgery, arXiv:1602.05951.

[12] P. Putrov, J. Wang, and S.-T. Yau, Braiding statistics and link invariants of Bosonic/Fermionic topological quantum matter in $2+1$ and $3+1$ dimensions, Ann. Phys. (Amsterdam) 384, 254 (2017).

[13] J. Wang, K. Ohmori, P. Putrov, Y. Zheng, Z. Wan, M. Guo, H. Lin, P. Gao, and S.-T. Yau, Tunneling topological Vacua via extended operators: (Spin-)TQFT spectra and boundary deconfinement in various dimensions, Prog. Theor. Exp. Phys. 2018, 053A01 (2018).

[14] J. C. Wang, Aspects of symmetry, topology and anomalies in quantum matter., Ph.D. thesis, Massachusetts Institute of Technology, 2015.

[15] M. Guo, K. Ohmori, P. Putrov, Z. Wan, and J. Wang, Fermionic finite-group gauge theories and interacting symmetric/crystalline orders via Cobordisms, arXiv: 1812.11959.
[16] J. Wang, X.-G. Wen, and S.-T. Yau, Quantum statistics and spacetime topology: Quantum surgery formulas, Ann. Phys. (Amsterdam) 409, 167904 (2019).

[17] Fractional Statistics and Anyon Superconductivity, edited by F. Wilczek (World Scientific Publishing Co., Inc., Teaneck, NJ, 1990).

[18] C. Wang and M. Levin, Braiding Statistics of Loop Excitations in Three Dimensions, Phys. Rev. Lett. 113, 080403 (2014).

[19] S. Jiang, A. Mesaros, and Y. Ran, Generalized Modular Transformations in $(3+1)$ D Topologically Ordered Phases and Triple Linking Invariant of Loop Braiding, Phys. Rev. X 4, 031048 (2014).

[20] J. C. Wang and X.-G. Wen, Non-Abelian string and particle braiding in topological order: Modular $\operatorname{SL}(3, Z)$ representation and $(3+1)$-dimensional twisted gauge theory, Phys. Rev. B 91, 035134 (2015).

[21] J. C. Wang, Z.-C. Gu, and X.-G. Wen, Field Theory Representation of Gauge-Gravity Symmetry-Protected Topological Invariants, Group Cohomology and Beyond, Phys. Rev. Lett. 114, 031601 (2015).

[22] Z.-C. Gu, J. C. Wang, and X.-G. Wen, Multi-kink topological terms and charge-binding domain-wall condensation induced symmetry-protected topological states: Beyond Chern-Simons/BF theory, Phys. Rev. B 93, 115136 (2016).

[23] P. Ye and Z.-C. Gu, Topological quantum field theory of three-dimensional bosonic Abelian-symmetry-protected topological phases, Phys. Rev. B 93, 205157 (2016).

[24] A. Tiwari, X. Chen, and S. Ryu, Wilson operator algebras and ground states for coupled BF theories, Phys. Rev. B 95, 245124 (2017).

[25] H. He, Y. Zheng, and C. von Keyserlingk, Field theories for gauged symmetry protected topological phases: Abelian gauge theories with non-Abelian quasiparticles, Phys. Rev. B 95, 035131 (2017).

[26] Q.-R. Wang, M. Cheng, C. Wang, and Z.-C. Gu, Topological quantum field theory for Abelian topological phases and loop braiding statistics in $(3+1)$-dimensions, Phys. Rev. B 99, 235137 (2019).

[27] R. Dijkgraaf and E. Witten, Topological gauge theories and group cohomology, Commun. Math. Phys. 129, 393 (1990).

[28] P. Ye and Z.-C. Gu, Vortex-Line Condensation in Three Dimensions: A Physical Mechanism for Bosonic Topological Insulators, Phys. Rev. X 5, 021029 (2015).

[29] X. Chen, Z.-C. Gu, Z.-X. Liu, and X.-G. Wen, Symmetry protected topological orders and the group cohomology of their symmetry group, Phys. Rev. B 87, 155114 (2013).

[30] T. Senthil, Symmetry-protected topological phases of quantum matter, Annu. Rev. Condens. Matter Phys. 6, 299 (2015). 
[31] X.-G. Wen, Colloquium: Zoo of quantum-topological phases of matter, Rev. Mod. Phys. 89, 041004 (2017).

[32] X.-G. Wen, Choreographed entanglement dances: Topological states of quantum matter, Science 363, eaal3099 (2019).

[33] X.-L. Qi, T. L. Hughes, and S.-C. Zhang, Topological field theory of time-reversal invariant insulators, Phys. Rev. B 78, 195424 (2008).

[34] X.-G. Wen, Construction of bosonic symmetryprotected-trivial states and their topological invariants via $G \times S O(\infty)$ non-linear $\sigma$-models, Phys. Rev. B 91, 205101 (2015).

[35] M. A. Metlitski, $S$-duality of $u(1)$ gauge theory with $\theta=\pi$ on non-orientable manifolds: Applications to topological insulators and superconductors, arXiv: 1510.05663.

[36] E. Witten, Fermion path integrals and topological phases, Rev. Mod. Phys. 88, 035001 (2016).

[37] E. Witten, The "Parity" anomaly on an unorientable manifold, Phys. Rev. B 94, 195150 (2016).

[38] M. Guo, P. Putrov, and J. Wang, Time reversal, SU(N) Yang-Mills and cobordisms: Interacting topological superconductors/insulators and quantum spin liquids in $3+1 D$, Ann. Phys. (Amsterdam) 394, 244 (2018).

[39] A. Kapustin, Symmetry protected topological phases, anomalies, and Cobordisms: Beyond group cohomology, arXiv:1403.1467.

[40] A. Kapustin, R. Thorngren, A. Turzillo, and Z. Wang, Fermionic symmetry protected topological phases and Cobordisms, J. High Energy Phys. 12 (2015) 052.

[41] D. S. Freed and M. J. Hopkins, Reflection positivity and invertible topological phases, arXiv:1604.06527.

[42] R. Thom, Quelques propriétés globales des variétés différentiables, Commentarii mathematici Helvetici 28, 17 (1954).

[43] S. Galatius, I. Madsen, U. Tillmann, and M. Weiss, The homotopy type of the cobordism category, Acta Math. 202, 195 (2009).

[44] A. Kapustin and R. Thorngren, Higher symmetry and gapped phases of gauge theories, arXiv:1309.4721.

[45] E. Sharpe, Notes on generalized global symmetries in QFT, Fortschr. Phys. 63, 659 (2015).

[46] A. Kapustin and R. Thorngren, Fermionic SPT phases in higher dimensions and bosonization, J. High Energy Phys. 10 (2017) 080.

[47] Y. Tanizaki, T. Misumi, and N. Sakai, Circle compactification and 't Hooft anomaly, J. High Energy Phys. 12 (2017) 056.

[48] C. Cordova, T. T. Dumitrescu, and K. Intriligator, Exploring 2-group global symmetries, J. High Energy Phys. 02 (2019) 184.

[49] C. Delcamp and A. Tiwari, From gauge to higher gauge models of topological phases, J. High Energy Phys. 10 (2018) 049.

[50] F. Benini, C. Cordova, and P.-S. Hsin, On 2-group global symmetries and their anomalies, J. High Energy Phys. 03 (2019) 118.

[51] K. Yonekura, On the cobordism classification of symmetry protected topological phases, Commun. Math. Phys. 368, 1121 (2019).

[52] C. Zhu, T. Lan, and X.-G. Wen, Topological non-linear $\sigma$ model, higher gauge theory, and a realization of all $3+1 \mathrm{D}$ topological orders for boson systems, Phys. Rev. B 100, 045105 (2019).

[53] C. Delcamp and A. Tiwari, On 2-form gauge models of topological phases, J. High Energy Phys. 05 (2019) 064.

[54] J. Wang, X.-G. Wen, and E. Witten, Symmetric Gapped Interfaces of SPT and SET States: Systematic Constructions, Phys. Rev. X 8, 031048 (2018).

[55] Z. Wan and J. Wang, Adjoint $\mathrm{QCD}_{4}$, symmetry-enriched TQFT and higher symmetry-extension, Phys. Rev. D 99, 065013 (2019).

[56] E. Witten, An SU(2) anomaly, Phys. Lett. 117B, 324 (1982).

[57] J. Wang, X.-G. Wen, and E. Witten, A new SU(2) anomaly, J. Math. Phys. (N.Y.) 60, 052301 (2019).

[58] J. Wang, L. H. Santos, and X.-G. Wen, Bosonic Anomalies, induced fractional quantum numbers and degenerate zero modes: The anomalous edge physics of symmetry-protected topological states, Phys. Rev. B 91, 195134 (2015).

[59] Z. Komargodski, A. Sharon, R. Thorngren, and X. Zhou, Comments on Abelian Higgs models and persistent order, SciPost Phys. 6, 003 (2019).

[60] M. A. Metlitski and R. Thorngren, Intrinsic and emergent anomalies at deconfined critical points, Phys. Rev. B 98, 085140 (2018).

[61] N. E. Steenrod, Products of cocycles and extensions of mappings, Ann. Math. 48, 290 (1947).

[62] O. Aharony, N. Seiberg, and Y. Tachikawa, Reading between the lines of four-dimensional gauge theories, J. High Energy Phys. 08 (2013) 115.

[63] C. Wang and T. Senthil, Time-Reversal Symmetric $U(1)$ Quantum Spin Liquids, Phys. Rev. X 6, 011034 (2016).

[64] L. Zou, C. Wang, and T. Senthil, Symmetry enriched U(1) quantum spin liquids, Phys. Rev. B 97, 195126 (2018).

[65] N. Seiberg, J. Wang, and E. Witten, (to be published).

[66] M. Guo, P. Putrov, and J. Wang, (to be published).

[67] J. Wess and B. Zumino, Consequences of anomalous Ward identities, Phys. Lett. 37B, 95 (1971).

[68] E. Witten, Global aspects of current algebra, Nucl. Phys. B223, 422 (1983).

[69] A. Kapustin and N. Seiberg, Coupling a QFT to a TQFT and duality, J. High Energy Phys. 04 (2014) 001.

[70] G. 't Hooft, On the phase transition towards permanent quark confinement, Nucl. Phys. B138, 1 (1978).

[71] C. B. Allendorfer and J. J. Eells, On the cohomology of smooth manifolds, Commentarii mathematici Helvetici 32, 165 (1958).

[72] A. Kapustin and R. Thorngren, Fermionic SPT phases in higher dimensions and bosonization, J. High Energy Phys. 10 (2017) 080.

[73] J. Wang and X.-G. Wen, A non-perturbative definition of the Standard Models, arXiv:1809.11171.

[74] A. Kapustin and R. Thorngren, Topological field theory on a lattice, discrete theta-angles and confinement, Adv. Theor. Math. Phys. 18, 1233 (2014).

[75] A. Debray, The comparison of two cohomology operations (2017), https://web.ma.utexas.edu/users/a.debray/lecture_ notes/cobordism_approach.pdf.

[76] P.-S. Hsin and S.-H. Shao, Lorentz Symmetry Fractionalization and Dualities in $(2+1) d$, arXiv:1909.07383. 
[77] C. Wang and T. Senthil, Interacting fermionic topological insulators/superconductors in three dimensions, Phys. Rev. B 89, 195124 (2014).

[78] P.-S. Hsin, H. T. Lam, and N. Seiberg, Comments on oneform Global symmetries and their gauging in $3 \mathrm{~d}$ and $4 \mathrm{~d}$, SciPost Phys. 6, 039 (2019).

[79] A. Vishwanath and T. Senthil, Physics of Three Dimensional Bosonic Topological Insulators: Surface Deconfined Criticality and Quantized Magnetoelectric Effect, Phys. Rev. X 3, 011016 (2013).

[80] A. Y. Kitaev, Fault-tolerant quantum computation by anyons, Ann. Phys. (Amsterdam) 303, 2 (2003).

[81] Y. Hu, Y. Wan, and Y.-S. Wu, Twisted quantum double model of topological phases in two dimensions, Phys. Rev. B 87, 125114 (2013).

[82] Y. Wan, J. C. Wang, and H. He, Twisted gauge theory model of topological phases in three dimensions, Phys. Rev. B 92, 045101 (2015).

[83] M. A. Kervaire, Relative characteristic classes, Am. J. Math. 79, 517 (1957).

[84] K. Ohmori, 't Hooft anomaly, symmetry breaking, gaplessness, https://sis-pc15.ulb.ac.be/event/2/contributions/30/ attachments/35/130/6_K_Ohmori.pdf, https://livestream.com/ streaming/events/8742238/videos/193666407 (2019).

[85] C. Cordova and K. Ohmori, Anomaly obstructions to symmetry preserving gapped phases, (to be published).

[86] M. M. Anber and E. Poppitz, Two-flavor adjoint QCD, Phys. Rev. D 98, 034026 (2018).
[87] C. Cordova and T. T. Dumitrescu, Candidate phases for $\mathrm{SU}(2)$ adjoint $\mathrm{QCD}_{4}$ with two flavors from $\mathcal{N}=2$ supersymmetric Yang-Mills theory, arXiv:1806.09592.

[88] Z. Bi and T. Senthil, An Adventure in Topological Phase Transitions in 3 + 1-D: Non-Abelian Deconfined Quantum Criticalities and a Possible Duality, Phys. Rev. X 9, 021034 (2019).

[89] P.-S. Hsin and A. Turzillo, Symmetry-enriched quantum spin liquids in $(3+1) d$, arXiv:1904.11550.

[90] S.-Q. Ning, L. Zou, and M. Cheng, Fractionalization and anomalies in symmetry-enriched $\mathrm{U}(1)$ gauge theories, arXiv:1905.03276.

[91] R. Gompf and J. Wang (to be published).

[92] J. Wang, New Higher Anomalies and 3+1D Quantum Matter: From 4d Yang-Mills Gauge Theory to $2 \mathrm{~d} \mathbb{C P}^{N}$ Sigma Model; Quantum 4d Yang-Mills and TimeReversal Symmetric 5d TQFT: New Higher Anomalies to Anyonic-String/Brane Induced Topological Link Invariants (2019).

[93] J. Wang, Anyonic-String/Brane Träumerei: Quantum 4d Yang-Mills Gauge Theories and Time-Reversal Symmetric 5d TQFT, https://video.ias.edu/highenergy/2019/ 0419-JuvenWang, https://www.youtube.com/watch?v= GUMheJboWBs (2019).

Correction: A misprint introduced during the production process has been fixed in Eq. (2.3). The wrong acronyms were used after Eq. (8.17) and have been fixed. 